A reversal of fate: unravelling the role of central 5-HT in cardiorespiratory control in neonatal and adult rodents

\begin{tabular}{c}
$\begin{array}{c}\text { A Dissertation } \\
\text { presented to }\end{array}$ \\
the Faculty of the Graduate School \\
at the University of Missouri-Columbia \\
\hline In Partial Fulfillment \\
Of the Requirements for the Degree \\
Doctor of Philosophy
\end{tabular}

by

Jennifer L. Magnusson

Dr. Kevin J. Cummings, Dissertation Supervisor

July, 2018 
The undersigned, appointed by the Dean of the Graduate School, have examined the dissertation entitled:

\section{A reversal of fate: unravelling the role of central 5-HT in cardiorespiratory control in neonatal and adult rodents}

Presented by Jennifer L. Magnusson

A candidate for the degree of Doctor of Philosophy

And hereby certify that in their opinion is worthy of acceptance.

Kevin J. Cummings

Eileen M. Hasser

David D. Kline

Lorin S. Milescu 
Dedication

For my family and friends. 


\section{ACKNOWLEDGEMENTS}

I am deeply grateful to my dissertation chair, Dr. Kevin J. Cummings, who has been a truly exceptional advisor. By providing guidance and direction, and promoting good academic discussion, I developed into a stronger and more passionate researcher. He gave me opportunities to learn and provided insights that made my academic journey far more fruitful than it otherwise would have been. I appreciate his support, guidance, and encouragement, and know that he has been instrumental in the completion of my degree and my success.

I sincerely thank my committee members, Dr. Eileen Hasser, Dr. David Kline, and Dr. Lorin Milescu for all of their advice and direction. I was lucky to have a wonderful committee and never dreaded my meetings. I would especially like to thank Dr. Hasser, who helped me to re-think what I know about cardiovascular physiology. Her methodological insights also improved my dissertation analysis. I also sincerely thank Dr. Cheryl Heesch for her valuable insight, helpful discussions, and for providing several reagents that contributed to my last project.

I also thank members of our lab, past and present. They provided me with a wonderful scientific environment and inspired my research. Jane Chen was wonderful to work with, Jacob Young and our VRSP students (Emily and Marina) were very ambitious and intuitive in the lab, and Jennifer Cornelius-Green's love of pink was truly enjoyable.

I greatly appreciate my family and friends who supported me through the tough times and the good ones. My fellow PhD students supported and encouraged me through this process, and I am glad to have shared this experience with them. In particular, I benefited from many fruitful discussions about my dissertation with Lauren B. I also 
greatly appreciate the support from the administrative staff in the department; both Tommy and Karol made my experience much smoother, and more eco-friendly! 


\section{TABLE OF CONTENTS}

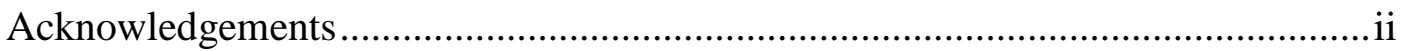

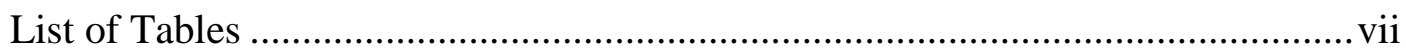

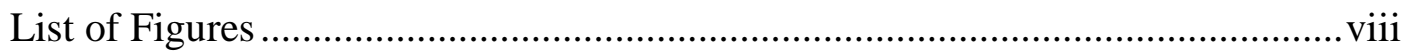

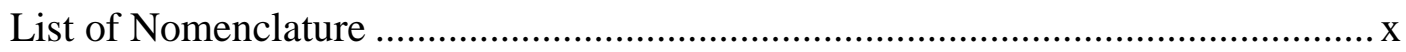

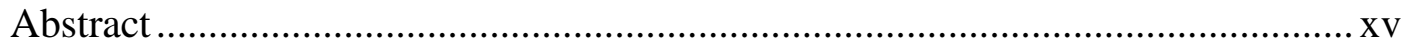

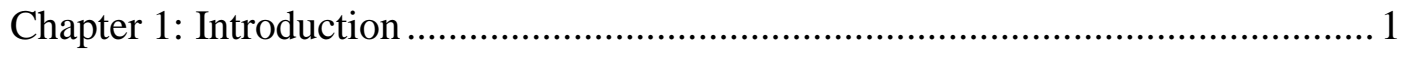

1.1 - Dissertation Overview …………………………......................... 1

1.2 - Overview of cardiorespiratory and autonomic function ...................... 1

1.3 - Cardiovascular Control .................................................................. 3

1.4 - Respiratory Control................................................................... 8

1.5 - Overview of the sleep-wake cycle and arousal from sleep................. 18

1.6 - Cardiorespiratory function during sleep …………………………..... 23

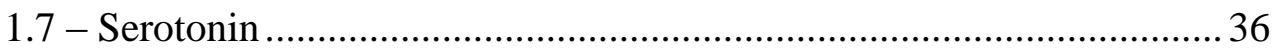

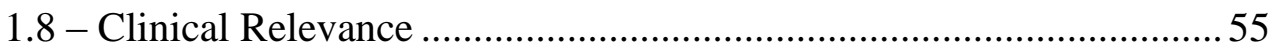

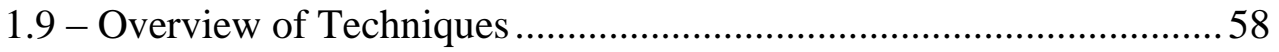

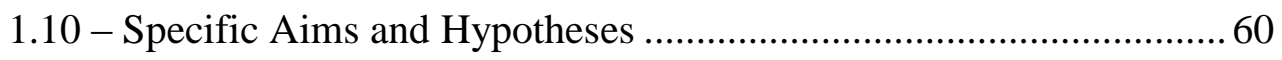

Chapter 2: Plasticity in breathing and arterial blood pressure following acute intermittent hypercapnic hypoxia in infant rat pups with a partial loss of 5-

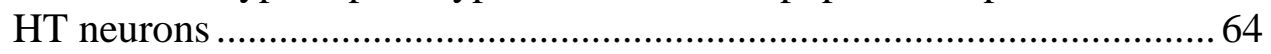

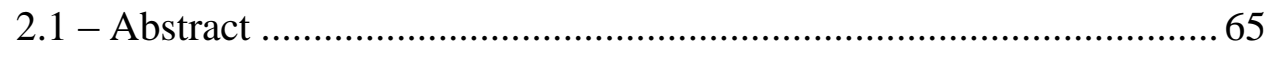

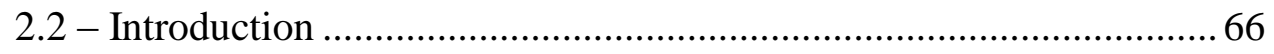

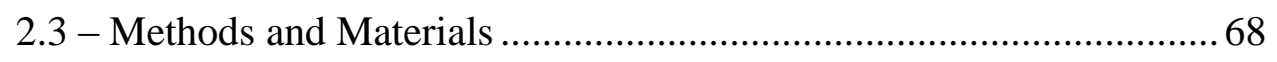

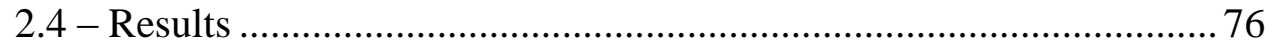

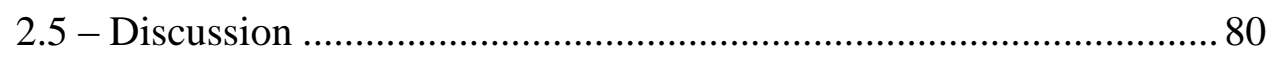


2.7 - Perspectives and Significance .............................................. 85

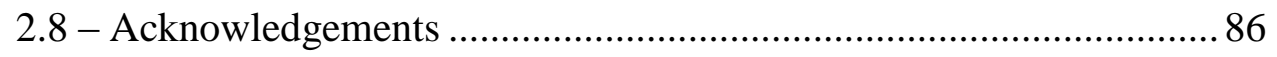

Chapter 3: Central serotonin and the control of arterial blood pressure and heart rate in infant rats: influence of sleep state and sex .......................................... 100

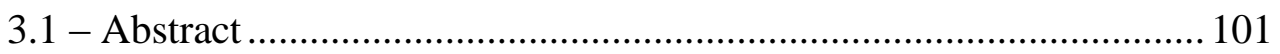

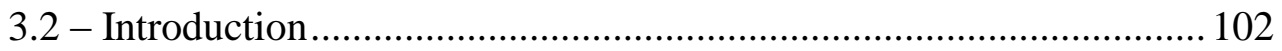

3.3 - Methods and Materials............................................................ 103

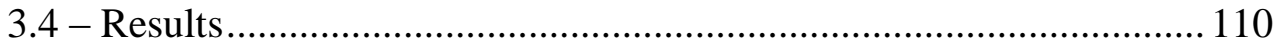

3.5 - Discussion ..................................................................... 113

3.6 - Perspectives and Significance............................................... 117

Chapter 4: Central serotonin prevents the development of hypertension in middleaged male rats: unravelling the mechanisms underlying a potentially novel form of

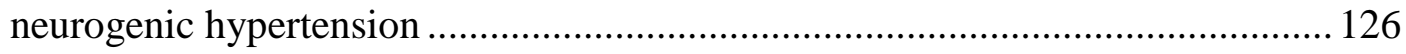

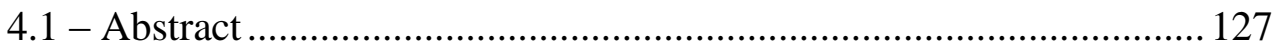

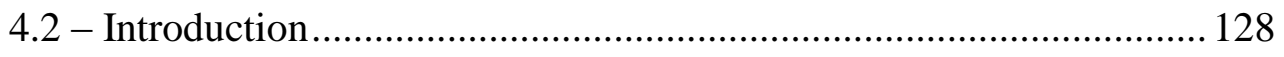

4.3 - Methods and Materials............................................................ 130

4.4 - Results........................................................................... 136

4.5 - Discussion ...................................................................... 147

4.6 - Perspectives and Significance .............................................. 157

4.7 - Acknowledgements ........................................................... 158

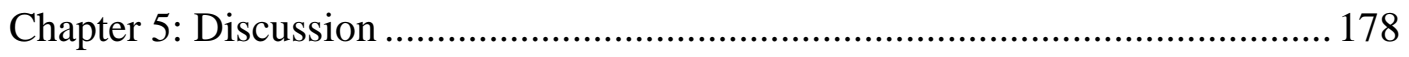

5.1: The role of central 5-HT in the control of ABP during sleep in neonatal rodents 179

5.2 - The role of central 5-HT on the control of ABP during sleep in adult rodents 187

5.3 - The role of central 5-HT in the control of ABP across development .... 
5.4 - Summary and Potential Future Studies ...................................... 198

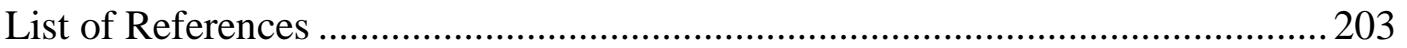

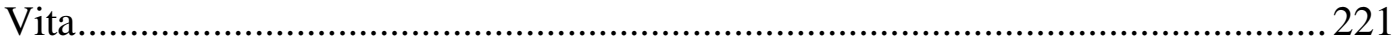




\section{LIST OF TABLES}

Table 1.1 - Summary of Sleep/Wake EEG and regulatory system activity ....................26

Table 2.1 - Summary of resting cardiorespiratory variables in saline and 5,7-DHT treated neonatal rats .88

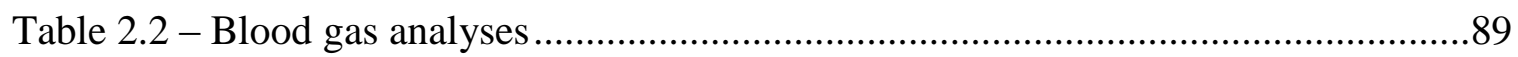

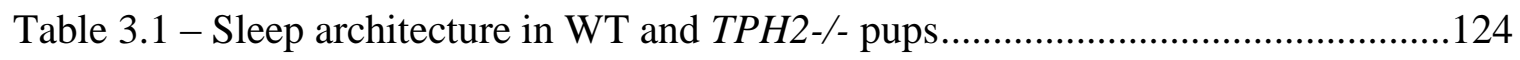

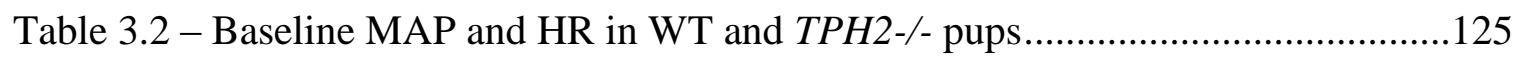

Table 4.1 - Sleep architecture in male WT and TPH2-/- adult rats.............................167

Table 4.2 - Sleep architecture in female WT and TPH2-/- adult rats...........................168

Table 4.3 - Baseline cardiovascular variables in male WT and TPH2-/- adult rats ........169

Table 4.4 - Baseline cardiovascular variables in female WT and TPH2-/- adult rats.....170

Table 4.5 - Baseline respiratory variables in male WT and TPH2-/- adult rats ..............171

Table 4.6 - Baseline respiratory variables in female WT and TPH2-/- adult rats...........172 


\section{LIST OF FIGURES}

Figure 1.1 - Schematic of PSNS and SNS activation of ABP and HR .............................

Figure 1.2 - Schematic of wake and sleep-promoting neurons ..................................22

Figure 1.3 - Schematic of sleep/wake regulatory systems ..........................................25

Figure 1.4 - Summary of the functional interactions between forebrain projecting wake-

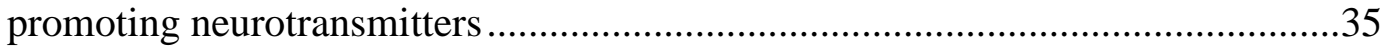

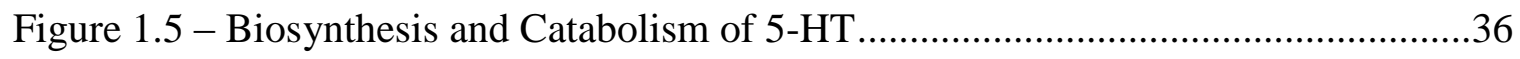

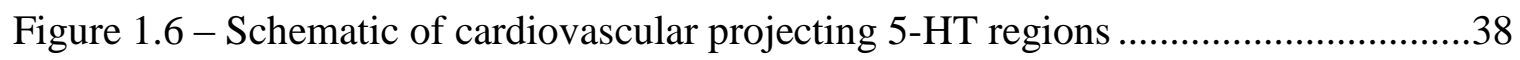

Figure 1.7 - Schematic of synaptic 5 -HT handling ............................................... 41

Figure 2.1 - Immunohistochemical analyses of medullary slices ...............................90

Figure 2.2 - Representative traces showing the cardiovascular response to an acute episode

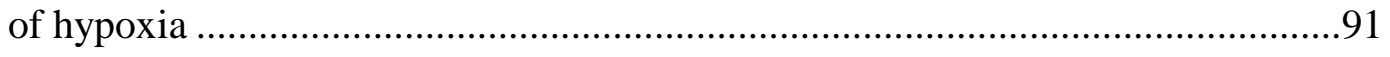

Figure 2.3 - Cardiovascular responses to acute intermittent hypoxia (Group 1) ............92

Figure 2.4 - Respiratory responses to acute intermittent hypoxia (Group 1) ..................93

Figure 2.5 - Cardiovascular responses to acute intermittent hypercapnia (Group 2).......94

Figure 2.6 - Respiratory responses to acute intermittent hypercapnia (Group 2) ............95

Figure 2.7 - Representative traces showing the cardiorespiratory responses to hypercapnic

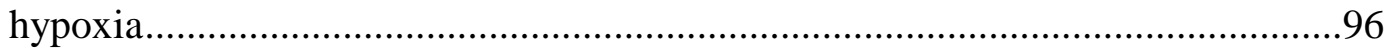

Figure 2.8 - Cardiovascular responses to acute intermittent hypercapnic hypoxia (Group 3)

Figure 2.9 - Representative blood pressure and respiratory traces pre- and post- acute intermittent hypercapnic hypoxia ..........................................................98

Figure 2.10 - Respiratory responses to acute intermittent hypercapnic hypoxia (Group 3)

Figure 3.1 - Example Traces of Arterial Pulse Pressure (PP) and nuchal electromyographic (EMG) records in neonatal rodents. 119

Figure 3.2 - Cardiovascular responses during sleep in neonatal rodents .120 
Figure 3.3 - Typical responses of MAP and HR to phentolamine in neonatal rodents...121 Figure 3.4 - Typical responses of MAP and HR to atropine and atenolol in neonatal rodents

Figure 3.5 - Summary of main findings .123

Figure 4.1 - Example Traces of Electromyography, Electroencephalograph, Mean Arterial Pressure (MAP), Heart Rate (HR) and Tidal Volume $\left(\mathrm{V}_{\mathrm{T}}\right)$ during NREM and REM

Figure 4.2 - MAP during sleep in adult rodents 160

Figure 4.3 - HR during sleep in adult rodents 161

Figure 4.4 - Typical responses of MAP and HR to ganglionic blockade in adult rodents.

Figure 4.5 - Time control of MAP and HR following ganglionic blockade in adult rodents

Figure 4.6 - Typical responses to Angiotensin Receptor blockade after ganglionic blockade in adult rodents. .164

Figure 4.7 - Typical responses to Angiotensin Receptor blockade prior to ganglionic blockade in adult rodents..... .165

Figure 4.8 - Example traces and data representing Extreme Hypertensive Events (EHE's) during REM sleep in adult rodents ........................................................ 166

Figures 4.9-4.13 - Supplemental Figures .......................................................... 173

Figure 5.1 - Schematic diagram showing proposed 5-HT projections to the HPA axis in the development of hypertension in 5-HT deficiency. .202 


\section{LIST OF NOMENCLATURE}

5,6-DHT: 5,6-dihydroxytryptamine

5,7-DHT: 5,7-dihydroxytryptamine

5-HT: 5-hydroxytryptamine; serotonin

5-HIAA: 5-hydroxyindoeacetic acid

5-HTP: 5-hydroxytryptophan

5-HTT: 5-HT transporter

6-FL: 6-fluorotryptophan

6-OHDA: 6-hydroxydopamine

ABP/BP: arterial blood pressure

Ach: acetylcholine

ACTH: adrenocorticotropic hormone

AIHH: acute intermittent hypercapnic hypoxia

Ang II: Angiotensin II

ARAS: ascending reticular activating system

AS: active sleep (neonatal rodents)

AT1: angiotensin receptor type I

AT2: angiotensin receptor type 2

AVP: vasopressin

BDNF: brain-derived neurotrophic factor

BF: basal forebrain

BötC: Bötzinger complex

CBS: cystathionine $\beta$-lyase 
CNS: central nervous system

CNS: central nervous system

CO: carbon monoxide

$\mathrm{CO}_{2}$ : carbon dioxide

CSE: cystathionine $\gamma$-lyase

CVLM/RVLM: caudal and rostral ventrolateral medulla

CVN: cardiac vagal neuron

DBH: dopamine $\beta$-hydroxylase

DMX: dorsal motor nucleus of the vagus

DOCA: deoxycorticosterone

DOI: 2,5-Dimethoxy-4-iodoamphetamine

DRN: dorsal raphe neurons

E: epinephrine

EH: Essential Hypertension

EMG: electromyography

EEG: electroencephalography

fB: breathing frequency

GABA: $\gamma$-Aminobutyric acid

$\mathrm{H}_{2} \mathrm{~S}$ : hydrogen sulfide

HCVR: hypercapnic ventilatory response

HO: heme oxigenase

HPA: hypothalamic pituitary axis

HR: heart rate 
$\mathrm{HR}_{\mathrm{INT}}$ : Intrinsic heart rate

HVR: hypoxic ventilatory response

i.c.v.: Intracerebroventricular injection

i.v.: intravenous

$\mathrm{IH}$ : intermittent hypoxia

IML: intermediolateral nucleus of the spinal cord

KFN: Kölliker Fuse Nucleus

LC-NA: Locus Coeruleus noradrenaline neurons

LdT: laterodorsal nuclei

LPBN: Lateral Parabrachial Nucleus

LPGi: lateral paragigantocellular nucleus

LTF: long-term facilitation

MAO: monoamine oxidase

MAP: mean arterial pressure

MRN: median raphe nucleus

NA: nucleus ambiguous

NE: norepinephrine

NET: norepinephrine transporter

NO: nitrous oxide

NOS: nitric oxide synthase (endothelial: eNOS; neuronal: nNOS)

NPY: neuropeptide Y

NREM: non-rapid eye movement sleep

NRM: raphe magnus 
NRO: raphe obscurus

NRPa: raphe pallidus

NTS: nucleus of the solitary tract

$\mathrm{O}_{2}$ : Oxygen

OX: orexin

$\mathrm{PaCO}_{2}$ : partial pressure of carbon dioxide

$\mathrm{PaO}_{2}$ : partial pressure of oxygen

p-CPA: para-chlorophenylalanine

PE: phenylephrine

PGO: pontogeniculate-occipital cortex

pLTF: phrenic long-term facilitation

PNMT: phenylethanolamine-N-methyltransferase

PNS: peripheral nervous system

PPT: pedunculopontine tegmental nucleus

pre-BötC: pre-Bötzinger complex

PSNS: parasympathetic nervous system

PVN: paraventricular nucleus of the hypothalamus

QS: quiet sleep (neonatal rodents)

REM: rapid eye movement sleep

RR: respiratory rate

RTN: retrotrapezoid nucleus

SCN: suprachiasmatic nucleus of the hypothalamus

SFO: subfornical organ 
SIDS: sudden infant death syndrome

sLTF: sympathetic long-term facilitation

SNA: sympathetic nerve activity

SNP: sodium nitroprusside

SNS: sympathetic nervous system

SubC: subcoeruleus

sBP: systolic blood pressure

dBP: diastolic blood pressure

TMN: Tuberomammillary nucleus

TPH2: tryptophan hydroxylase 2

TRH: thyrotropin releasing hormone

TrkB: tyrosine kinase B

$\mathrm{V}_{\mathrm{E}} / \mathrm{VO}_{2}$ : metabolic equivalent

$\mathrm{V}_{\mathrm{E}}$ : minute ventilation

VIP: vasoactive intestinal peptide

vlPAG: ventral periaqueductal gray

VLPO: ventrolateral preoptic nucleus

VMAT: vesicular monamine transporter

$\mathrm{VO}_{2}$ : metabolic rate

$\mathrm{V}_{\mathrm{T}}$ : tidal volume

vLF\%: very low frequency power spectral analysis

LF\%: low frequency power spectral analysis 


\begin{abstract}
We seek to address the extent to which a specific loss of 5-hydroxytryptamine (5HT) affects the control of respiration, arterial blood pressure (ABP) and heart rate (HR) across vigilance-states based on existing evidence suggesting that 5-HT defects increase the risk for Sudden Infant Death Syndrome (SIDS) and neurogenic hypertension. SIDS is the leading cause of infant mortality between 1 month and 1 year of age, occurs during sleep, and up to $70 \%$ of all SIDS cases have at least one 5-HT system abnormality. Neonatal rodents lacking central 5-HT exhibit severe apneas, and a reduced ABP and HR. Central 5-HT has been implicated in the etiology of neurogenic hypertension, presumably due to projections of 5-HT neurons within the midline raphe to vagal and presympathetic regions of the brain. However, data from studies examining the specific role of central 5-HT function is conflicting or inconclusive. Neurogenic hypertension accounts for more than $90 \%$ of all hypertensive cases and the normal fall in ABP that occurs during non-rapid eye movement sleep is absent in some patients with hypertension. Understanding the mechanisms associated with the development of hypertension is critical not only to lower blood pressure, but to lower its associated cardiovascular events. The purpose of this dissertation is to examine the role of central 5-HT in the control of ABP during sleep and reveal, mechanistically, the physiological role of 5-HT in the autonomic control of ABP in neonatal and adult rodents. The overarching hypothesis for this dissertation is that central 5-HT is required for the maintenance of $\mathrm{ABP}$ and autonomic tone at rest in both neonatal and adult rodents.
\end{abstract}




\section{CHAPTER 1: INTRODUCTION}

\section{1 - Dissertation Overview}

The cardiorespiratory system integrates a wide variety of sensory afferent inputs and controls a variety of efferent projections to maintain homeostasis. This dissertation seeks to identify the extent to which central serotonin (5-hydroxytryptamine, 5-HT) is involved in cardiorespiratory homeostasis in both infancy and adulthood, particularly in the regulation of mean arterial pressure (MAP) and heart rate (HR) at rest, during sleep, and the adaptations that follow intermittent hypoxia. Three studies (Chapters 2-4) were performed to investigate the overall hypothesis that central 5-HT maintains MAP and HR at rest and during sleep in neonatal and adult rodents and is required for the plasticity that follows intermittent hypoxia in neonatal rodents.

\section{$\underline{1.2}$ - Overview of cardiorespiratory and autonomic function}

Maintenance of breathing, MAP, and HR are among the most critical functions to life. Neural control of the cardiorespiratory system ensures that the metabolic demands of the body are met. There are two main sets of sensory receptors with respect to cardiorespiratory control. First, the baroreceptors, which are mechanoreceptors that sense changes in MAP, located in the carotid sinuses (at the bifurcation of the external and internal carotid arteries) and in the aortic arch. Second, the chemoreceptors, which sense oxygen content and $\mathrm{pH} / \mathrm{CO}_{2}$ of arterial blood, located in the carotid bodies (at the bifurcation of the common carotid artery) and in the aortic arch. Species differences in the presence of the aortic arch chemoreceptors exist; indeed, dogs and cats have aortic body chemoreceptors but they are generally absent in rabbits, rats, and mice [7]. When activated, 
action potentials from the baro- and chemo-receptors travel through the nodose and petrosal ganglia, respectively, ultimately traversing to the nucleus of the solitary tract (NTS), the first synapse of afferent information within the central nervous system (CNS). Afferent information from the baro- and chemo-receptors at the carotid sinuses/bodies travel via the glossopharyngeal nerve, whereas information from the aortic arch travels via the aortic depressor nerve. Decreased activity of the baroreceptors (in cases of low MAP) ultimately gives rise to an elevation of sympathetic and reduction in vagal efferent activity to increase cardiac output and systemic vascular resistance to ensure MAP remains constant. Similarly, following activation of the chemoreceptors in cases of low arterial $\mathrm{PO}_{2}$ or high arterial $\mathrm{PCO}_{2}$, there is a rise in the rate and depth of respiration to bring arterial $\mathrm{PO}_{2}$ and $\mathrm{PCO}_{2}$ within normal limits. Activation of the chemoreflex not only increases ventilation, but also increases sympathetic outflow to the muscle, splanchnic, and renal beds to increase blood pressure. In humans, cardiac sympathetic activity is also increased, which gives rise to an increase in HR and contractility. Thus, chemoreceptor activation is important in both the control of ventilation and cardiovascular function [7]. Overall, activation of the baroreceptors helps maintain MAP and HR while the chemoreceptors help maintain normal arterial $\mathrm{pH}, \mathrm{CO}_{2}$, and $\mathrm{O}_{2}[11,12]$.

Collections of afferent and efferent neurons link the CNS with visceral effectors that comprise the autonomic nervous system (sympathetic and parasympathetic divisions). Sympathetic activity is often referred to as the "fight-or-flight" response, while parasympathetic activity is referred to as the "rest-and-digest" response. Sympathetic innervation of the heart and blood vessels increases HR, cardiac contractility and elicits 
vasoconstriction. Alternatively, parasympathetic innervation inhibits cardiovascular function, reducing HR and cardiac contractility.

CNS coordination of the inspiratory and expiratory muscles, including the diaphragm, intercostal, and abdominal muscles, helps produce rhythmic inspiration and expiration. The spinal cord contains the cell bodies of the motor neurons that innervate these respiratory muscles. The phrenic nerve innervates the diaphragm, originating from phrenic motor nuclei within the C3-C5 region of the spinal cord in humans [13], C5-C6 in cats [14], and C3-C6 in rats [15]. The intercostal nerves originate between T1-T12 and the abdominal motor nerves originate from the T4-L3 region of the spinal column $[2,12,16]$.

\section{3 - Cardiovascular Control}

The medulla contains cell bodies for both arms of the autonomic nervous system. Parasympathetic (PSNS) control of HR stems from PSNS nuclei within the brainstem (e.g. the nucleus ambiguus and the dorsal motor nucleus of the vagus). Upon PSNS stimulation, the vagus releases acetylcholine (Ach) near the heart, which activates muscarinic Ach receptors to decrease HR. Anatomically, the vagus innervates both the SA and AV nodes, activating muscarinic receptors, of which there are three main subtypes. M1 is found mainly in the autonomic ganglia and the CNS, M2 is abundant in the heart, and M3 is found in smooth muscle and secretory cells [17]. While Ach is the primary neurotransmitter in the PSNS, vasoactive intestinal polypeptide (VIP) may also be co-released from parasympathetic terminals, as well as nitric oxide (NO) [2]. The sympathetic (SNS) arm plays a role in the control of both ABP and HR. SNS control of HR stems from presympathetic nuclei within the brainstem (e.g. the RVLM) as well as the sympathetic nerves 
that arise from the intermediolateral column of the spinal cord. Activation of the SNS elicits an increase in ABP through activation of $\alpha-1$ receptors on the vasculature, preferentially activated by norepinephrine. Activation of the SNS also elicits a rise in HR through $\beta-1$ receptors on the heart (SA node), which is equally activated by norepinephrine and epinephrine [18]. In rats, the inotropic (contractility) and chronotropic (rate) responses are primarily mediated via $\beta 1$-adrenergic receptors [19]. Activation of $\beta$-receptors can also elicit a rise in $\mathrm{ABP}$, mediated in part by elevating cardiac output, through chronotropic and inotropic mechanisms. Indeed, $\beta$-blockers were one of the most frequently prescribed antihypertensive agents until the 1990s [20,21]. While the sympathetic neurons primarily utilize norepinephrine as their primary neurotransmitter, other neuropeptides like neuropeptide Y (NPY) and galanin are co-released from sympathetic terminals. NPY and galanin act to decrease Ach release from adjacent PSNS terminals [2]. Similarly, PSNS release of Ach, released near adrenergic nerve endings in the heart, exerts an inhibitory effect on the release of norepinephrine and thus, reduces the amount of adrenergic neurotransmitter present near the heart [22]. Thus, there is an overall mutual modulation of HR between the SNS and PSNS. However, in adult mammals, vagal effects on the sinus node predominate over sympathetic effects [2]. These effects are simplified in Fig. 1.1. 

Parasympathetic Nuclei Presympathetic Nuclei
(e.g. NA; DMX)
(e.g. RVLM)

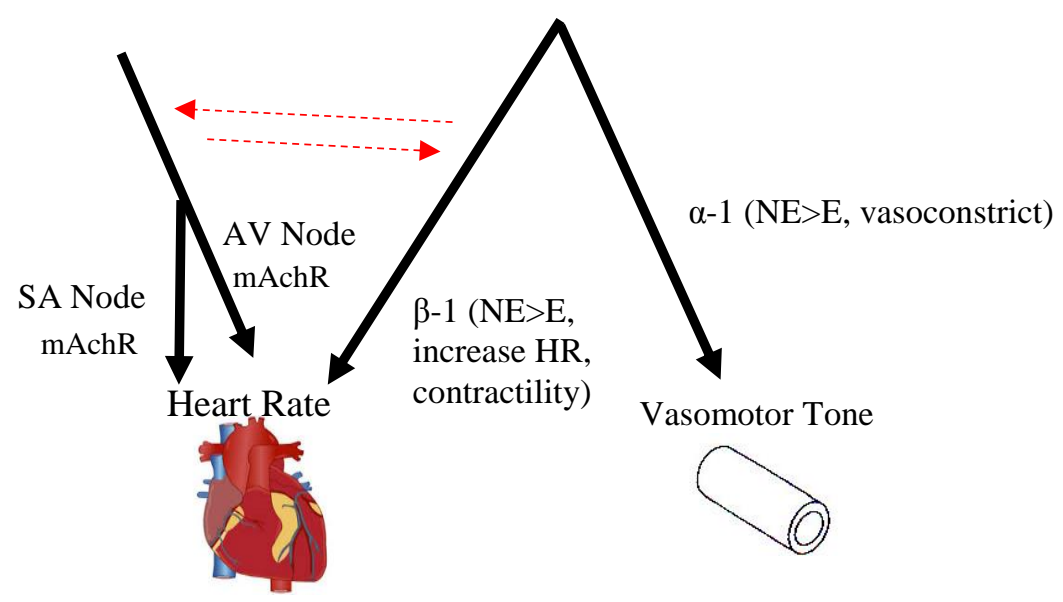

Fig. 1.1: Simplified schematic of PSNS and SNS activation of HR and ABP through muscarinic acetylcholine receptors (mAchR) and $\beta-1$ and $\alpha-1$ receptors, respectively. Red dashed lines indicate a mutual inhibitory effect of PSNS activity on SNS NA release and SNS inhibition of PSNS Ach activity [2].

MAP is tightly regulated but can fluctuate over the course of a given day. Both the afferent arm (e.g. the baro- and chemo-receptors) as well as circuitry within the CNS are responsible for helping remain MAP constant. Baroreceptors, located in the aortic arch and in the carotid sinuses at the bifurcation of the external and internal carotid arteries, send projections to the NTS via the aortic depressor and glossopharyngeal nerves, respectively. In general, activation of the baroreflex leads to inhibition of the SNS centers and excitation of the PSNS centers to produce an overall reduction in HR and stroke volume to mitigate the increased MAP. The opposite occurs during falls in pressure (hypotension) [11, 23]. While the baroreflex is essential for counteracting rapid changes in MAP, alteration of fluid volume helps determine the long-term level of MAP through the kidneys, by altering MAP through sodium handling and the regulation of blood volume. The CNS also plays a role in long-term control of ABP, but the exact mechanisms are not well understood [24]. 
Renin release can help maintain $\mathrm{ABP}$ within a normal range, centrally or peripherally, via activation of the Renin-Angiotensin-System (RAS). Renin release into the circulation by the kidneys can be stimulated by: sympathetic stimulation, hypotension, or decreased sodium delivery to the macula densa. Within the peripheral RAS, renal sympathetic nerve activity elicits renin secretion that leads to angiotensin II (Ang II) induced vasoconstriction. Ang II acts through one of two receptors, AT1 or AT2, but the importance of AT2 in the regulation of $\mathrm{ABP}$ is not completely understood. There is evidence that Ang II can activate the SNS by acting on the ganglia [25], at sympathetic nerve terminals [26], or by altering end-organ responsiveness [27], in addition to exerting its influence within the CNS [25]. Therefore, it is possible that Ang II could contribute to neural activation at any (or all) of these sites. Ang II also leads to the secretion of aldosterone (ALDO) or vasopressin (AVP), both of which mediate an increase in blood pressure. ALDO acts through expansion of blood volume through its actions at mineralocorticoid receptors in the distal tubules and collecting ducts of the nephron (influencing the reabsorption of sodium and excretion of potassium), whereas AVP elicits peripheral vasoconstriction and an increase in water reabsorption within the collecting ducts of the kidneys [28].

The RAS exists within the CNS as a distinctly separate system from the peripheral RAS; indeed, Ang II does not readily cross the BBB. However, brain RAS is not independent from the peripheral RAS as Ang II can interact with the brain RAS through the circumventricular organs (CVOs), special regions in the brain located around the third and fourth ventricles that lack the BBB. This provides a unique opportunity for the brain to sense changes in the concentration of hormones and ions within the circulation. AT1 
receptors are expressed in brain regions regulating autonomic responses, including the CVOs, PVN, ventrolateral medulla, and the NTS. Within these areas, the $\mathrm{AT}_{1 \mathrm{~A}}$ receptor is the most prevalent receptor subtype in rodents, and contributes to maintaining resting ABP and mediating pressor responses to Ang II administration. Similar to in the PNS, CNS AT2 receptor contribution to cardiovascular regulation remains elusive, despite its location in central cardiovascular regions. However, central administration of Ang II elicits AVP release predominately through AT1 receptors, but AT2 can also mediate the response [29].

The HPA axis plays an active role in the maintenance of homeostasis in the presence of aversive stimuli, including physiological stressors like hypotension, hypoxia, and hypovolemia. The principal effectors responsible for this homeostasis are the PVN, the pituitary gland, and the adrenal gland. The PVN contains neurons that synthesize and secrete corticotropin-releasing factor $(\mathrm{CRF})$, the principal regulator of the HPA. In response to stress, CRF activates its receptor in the pituitary gland, inducing the release of adrenocorticotropic hormone $(\mathrm{ACTH})$, which acts at the adrenal cortex to stimulate glucocorticoid (i.e. cortisol and corticosterone) synthesis and secretion. Glucocorticoids play an active role in the negative feedback mechanisms within the HPA axis to protect against prolonged activity. Many regions in the brain are important in regulating the HPA axis, including the NTS, hippocampus, and the lamina terminalis [30].

The rise in glucocorticoids typically following stress is typically thought of as advantageous to restore homeostasis. However, excess glucocorticoids, as seen in situations of chronic stress, have been implicated in the development of hypertension. The effects of glucocorticoids on BP may be regulated through the expression of glucocorticoid receptors (GR) on the vasculature. Indeed, glucocorticoid activation of GR's potentiates 
the effects of catecholamines and other vasoactivators on vascular smooth muscle via suppressing the production of vasodilators like NO. Further, mice treated with glucocorticoids develop hypertension by a mechanism involving GR inhibition of NO metabolites [31].

\section{4 - Respiratory Control}

The chief function of the lung is to exchange oxygen $\left(\mathrm{O}_{2}\right)$ and carbon dioxide $\left(\mathrm{CO}_{2}\right)$ between the blood and air to maintain normal partial pressures of oxygen $\left(\mathrm{PO}_{2}\right)$ and carbon dioxide $\left(\mathrm{PCO}_{2}\right)$. As described in detail in the following sections, various mechanisms contribute to the maintenance of an appropriate arterial $\mathrm{PO}_{2}\left(\mathrm{PaO}_{2}\right)$ and $\mathrm{PCO}_{2}\left(\mathrm{PaCO}_{2}\right)$, including muscles within the diaphragm to control breathing (effectors), sensors to detect changes of $\mathrm{PaO}_{2}$ and $\mathrm{PaCO}_{2}$ (chemoreceptors), and respiratory centers located within the brainstem, specifically in the pons and medulla, to centrally control inspiration and expiration.

\section{Effectors}

The control of ventilation requires various muscle groups working together, including muscles of the diaphragm as well as the intercostal and abdominal muscles. The inspiratory muscles are comprised of the diaphragm and external intercostal muscles. The expiratory muscles are comprised of the abdominal and the internal intercostal muscles. In rats, the phrenic nerves from cervical segments 3, 4, 5, and 6 supply the diaphragm and the intercostal nerves supply the intercostal muscles. The central controller plays a significant role in coordinating the actions of these muscles in a concerted fashion to maintain a normal 
ventilatory pattern [16]. The hypoglossal motoneurons also play a role in respiration at rest, in that they mediate contraction of the genioglossus muscle and contribute to regulation of upper airway patency [32]. The dorsal respiratory group, located bilaterally in the NTS, consists mainly of inspiratory neurons. These inspiratory neurons project to the contralateral spinal cord, are the principal initiators of phrenic nerve activity, and are responsible for maintaining diaphragmatic activity. Neurons within this region send many collateral fibers to the ventral respiratory group, which consists of both inspiratory and expiratory neurons, innervating the intercostal and abdominal muscles [33].

\section{Sensors}

Chemoreceptors respond to changes in the chemical composition of the blood, of which there are two types: peripheral and central. Peripheral chemoreceptors are located in the carotid bodies at the bifurcation of the common carotid artery and in the aortic bodies of the aortic arch. These receptors respond to decreases in arterial $\mathrm{PaO}_{2}$ and $\mathrm{pH}$ as well as increases in arterial $\mathrm{PaCO}_{2}$ [34], sending impulses through the glossopharyngeal and vagal nerves, via branches known as the carotid sinus and aortic nerves, respectively [7]. However, the aortic bodies don't play a significant role in peripheral chemoreception, at least in rats, unless the carotid bodies are denervated [35]. Central chemoreception stimulates breathing following an increase in brain $\mathrm{PaCO}_{2}$ and are involved in the minuteby-minute control of ventilation. Even a $1 \mathrm{mmHg}$ increase in $\mathrm{PaCO}_{2}$ increases ventilation in humans by $\sim 20-30 \%$ [34]. Central chemoreceptors also respond to changes in $\mathrm{pH}$, where an increase in $\mathrm{H}^{+}$increases ventilation and a decrease in $\mathrm{H}^{+}$reduces ventilation. It has been shown that even a 0.05 drop in $\mathrm{pH}$ can increase ventilation [16]. 
Within the carotid body, glomus cells depolarize in response to hypoxia and release neurotransmitters, including Ach, 5-HT, ATP, and substance $\mathrm{P}$ [36]. These neurotransmitters activate impulses in afferent fibers that travel to the medulla within the CNS via the carotid sinus nerve. The reflex response to chemoreceptor stimulation includes an increase in ventilation and an increase in sympathetic outflow to the muscle, splanchnic, and renal beds to increase blood pressure. In humans, cardiac sympathetic activity is also increased, which gives rise to an increase in HR and contractility. Thus, chemoreceptor activation is important in both the control of ventilation and cardiovascular function. The activation of chemoreceptors that increases sympathetic outflow to the vasculature compensates for direct vasodilating effects of hypoxia on blood vessels and redistributes blood flow to essential organs -- organs with the highest oxygen demands like the heart and brain [7].

More recent evidence also implicates gasotransmitters like nitric oxide (NO), carbon monoxide $(\mathrm{CO})$ and hydrogen sulfide $\left(\mathrm{H}_{2} \mathrm{~S}\right)$ in carotid body $\mathrm{O}_{2}$ sensing. Neurons containing nitric oxide synthase (NOS) are located within the petrosal ganglion, which innervates the carotid body. Neuronal NOS (nNOS) expression has also been observed in some ganglion cells of the carotid body whereas the endothelial NOS isoform (eNOS) is expressed in carotid body blood vessels [37]. Interestingly, the chemoreceptor responses differ with respect to nNOS and eNOS. Mice deficient in nNOS exhibit enhanced carotid body activity and an augmented ventilatory response to hypoxia [37, 38]. However, mice lacking eNOS have a significantly reduced peripheral chemoreceptor sensitivity and ventilatory response to hypoxia [39]. 
$\mathrm{CO}$ is synthesized by the enzyme heme oxygenase (HO) during degradation of heme in mammalian cells. Two forms of $\mathrm{HO}$ exist, with HO-2 being expressed by type I cells of the carotid bodies. As seen in experimental studies where inhibition of $\mathrm{HO}$ by $\mathrm{Zn}$ protoporphyrin-9 augments carotid body sensory activity and exogenous $\mathrm{CO}$ reverses the effect, it has been suggested that endogenously generated CO inhibits carotid body sensory activity [37]. Dysregulated CO signaling may lead to sleep apnea. Like sleep apnea patients, mice lacking HO-2 also exhibit high apnea and hypopnea indices during both NREM and REM sleep but not wakefulness. These mice also exhibited abnormal arterial blood gases, indicative of hypoxemia and hypercapnia. Further, since HO-2-null mice exhibit carotid body hypersensitivity, it may be that the elevated apnea indices in these mice are mediated by chronic activation of the carotid body chemoreflex [40].

$\mathrm{H}_{2} \mathrm{~S}$ can also regulate neuronal transmission as a gasotransmitter like $\mathrm{NO}$ and $\mathrm{CO}$. Generation of $\mathrm{H}_{2} \mathrm{~S}$ is mediated enzymatically via cystathionine $\gamma$-lyase (CSE, predominately peripheral) and cystathionine $\beta$-lyase (CBS, predominately in the brain). CSE is localized to type I cells of rat and mouse carotid bodies and hypoxia increases $\mathrm{H}_{2} \mathrm{~S}$ generation in the carotid body of wild-type but not CSE deficient mice. CSE knockout mice appear to display a selective impairment of carotid body responses to hypoxia but not $\mathrm{CO}_{2}$, demonstrating that in the carotid body, $\mathrm{H}_{2} \mathrm{~S}$ is an excitatory gasotransmitter [37]. It has also been shown that ablation of CSE normalizes breathing in HO-2 knockout mice suggesting that $\mathrm{H}_{2} \mathrm{~S}$ activation of the carotid body, and thus the chemoreflex, potentiates apneas in rodents with reduced CO [40]. In summary, these results implicate gasotransmitters in carotid body $\mathrm{O}_{2}$ sensing. 
Numerous brain manipulations (e.g. lesions) have produced results that show the specific CNS regions that contribute to long-term regulation of blood pressure, baroreflex function, breathing, and the chemoreflex. The following is a brief description of some of these regions.

\section{Nucleus of the Solitary Tract (NTS)}

The NTS is the primary site of termination and integration for peripheral sensory afferents, including the baroreceptors and chemoreceptors, and thus plays a role in both cardiovascular and respiratory control. Located in the dorsal brainstem, the NTS receives direct input from vagal and glossopharyngeal sensory nerves originating in the heart and large arteries. The primary neurotransmitter released at the NTS from sensory afferents is glutamate [41]. The NTS projects to several areas within the forebrain, brainstem and spinal cord to modulate cardiorespiratory control $[42,43]$. Within the NTS, there is an intricate network of both inhibitory (GABAergic) and excitatory (glutamatergic) neurons [44]. Both NMDA and non-NMDA (AMPA and kainate) receptors contribute to the actions of glutamate within the NTS. In vivo studies blocking NMDA receptors in the NTS with AP-5 blocks the bradycardic response following activation of the baroreflex with phenylephrine [45]. Similarly, blockade of non-NMDA receptors with 6,7dinitroquinoxaline-2,3-dione (DNQX) blocks the bradycardic response to electrical stimulation of the aortic depressor nerve [46]. NTS circuitry also contains neurons that are a part of the dorsal respiratory column, thus can contribute to eupneic breathing [47, 48]. Inhibition of ionotropic glutamatergic neurotransmission induces marked changes in respiratory activity [47]. In support of the involvement of the NTS in chemoreflex 
activation, agonism of $\gamma$-aminobutyric acid $\left(\mathrm{GABA}_{\mathrm{A}}\right)$ receptors with muscimol reduces the bradycardic response to chemoreflex activation [49]. Some reports indicate that 5-HT within the NTS can modulate the responses to baro- and chemo-reflex activation. For instance, agonism of 5- $\mathrm{HT}_{3}$ receptors has been shown to inhibit the cardiac component of the baroreflex and the Bezold-Jarisch reflex, without affecting their sympathetic components [50-52]. Similarly, antagonism of 5-HT $\mathrm{H}_{7}$ receptors attenuates the cardiovascular responses to chemo-, baro-, and cardiopulmonary reflexes [53]. Thus, the NTS is an important contributor to the ABP and HR responses to baro- and chemo-receptor activation and can contribute to respiratory control.

\section{Caudal and Rostral Ventrolateral Medulla (CVLM and RVLM)}

The caudal ventrolateral medulla (CVLM) plays a critical role in cardiovascular regulation, best known for its role in reflex control of MAP. Anatomically, the CVLM is within the baroreceptor reflex arc; receiving input from the NTS, the CVLM then projects to the RVLM, which contains neurons that excite sympathetic preganglionic neurons in the spinal cord [54]. Excitation of the CVLM produces a decrease in ABP due to decreased sympathetic nerve activity and decreased total peripheral resistance [54]. This effect is mediated by GABAergic CVLM neurons that project to the RVLM. In contrast, inhibition of the CVLM has been shown to increase ABP, consistent with its role as an inhibitor of the RVLM [55]. Thus, the CVLM is an important contributor to the baroreflex control of ABP.

In the early 1980s, the RVLM was identified as a key regulator in the control of ABP [56]. Now well regarded as a group of tonically active sympathoexcitatory neurons, 
the RVLM plays an essential role in the maintenance of sympathetic vasomotor activity and resting ABP. Indeed, studies have shown that blockade of the RVLM decreases ABP [55]. These neurons are also a site of convergence of central pathways that mediate cardiovascular responses evoked by stimulation of the baro- and chemo- receptors, as well as higher centers within the brain. Inputs to the RVLM are both excitatory (e.g. glutamate from the NTS) or inhibitory (e.g. GABA from the CVLM) [57].

The $\mathrm{C} 1$ neurons in the ventrolateral medulla have been implicated in many physiological processes, including the control of ABP. The defining characteristic of $\mathrm{C} 1$ adrenergic neurons is that they contain the catecholamine synthetic enzymes necessary for the production of epinephrine, including tyrosine hydroxylase (TH), dopamine $\beta$ hydroxylase (DBH), and phenylethanolamine-N-methyltransferase (PNMT). However, the bulbospinal -- brainstem-originating neurons that influence neurons within the spinal cord -- RVLM C1 adrenergic neurons also contain enkephalin, substance P, and calbindin. The projection pattern of $\mathrm{C} 1$ cells may be dependent on co-localized neuropeptides; for instance, upwards of $96 \%$ of NPY-containing C1 neurons project to the hypothalamus rather than the spinal cord. The non-C1 RVLM neurons make up 30-50\% of the bulbospinal neurons within the RVLM, which contain most of the peptides mentioned for C1 neurons, but exclude the presence of PNMT or TH [58].

The baroreflex arc sends projections from the NTS, through the CVLM and then onto the RVLM. The RVLM also receives chemoreflex input directly from the NTS, bypassing the CVLM [57]. Thus, the RVLM also plays a role in respiratory control. The neurons within the RVLM that contain the Phox2B transcription factor are putative chemoreceptors. Indeed, previous reports have indicated that mutations in the Phox2B lead 
to reduced $\mathrm{CO}_{2}$ chemosensitivity [59]. Photostimulation of these neurons through use of optogenetics produces increased breathing and a small sympathetically mediated rise in ABP [60]. Together, these studies point to the CVLM and RVLM as a key mediators in ABP and HR responses to baro- and chemo-receptor activation.

\section{Parasympathetic Nuclei}

Anatomical and electrophysiological studies of the cell bodies of cardiac preganglionic vagal neurons (CVN's) innervating the heart have been localized in the dorsal motor nucleus of the vagus (DMX) and the nucleus ambiguus (NA). These neurons are subject to tonic GABAergic inhibitory tone. The DMX and NA have distinct characteristics. The DMX has non-myelinated axons, a low firing rate and is unaffected by stimulation of the baroreceptors. The NA also has a low firing rate in anesthetized animals, but has small myelinated axons and exhibits an increase in firing following excitatory input from the baroreceptors. Furthermore, the activity of CVNs within the NA depend on the phase of breathing, being most active during post-inspiration; this feature does not occur in the DMX. In most mammals, the NA has a greater importance than the DMX in the control of HR $[61,62]$. This is especially true for dogs, cats, neonatal pigs, and rats, where tracer studies indicate that the majority of neurons innervating the heart were located in the external subnucleus of the NA. Although, the relative contribution of the NA and DMX in the control of HR does vary between species [62].

Pontine Respiratory Group -- Kölliker Fuse Nucleus and Lateral Parabrachial Nucleus 
Both the Kölliker Fuse Nucleus (KFN) and the lateral parabrachial nucleus (LPBN) are located within the parabrachial complex and are important in respiratory control. These regions include post-inspiratory neurons, which are important in the transition between inspiration and expiration as well as for maintaining the stability of respiratory rhythm [63].

The parabrachial/KF complex receives projections from the retrotrapezoid nucleus (RTN), described below, and the caudal regions of the NTS. These neuroanatomical features suggest a possible role for these regions in the chemoreflex [63]. Indeed, following hypoxia, hypercapnia, or stimulation of the carotid sinus nerve, c-Fos immunoreactivity, as a marker of neuronal activity, has shown that the KFN is intensely activated $[63,64]$ and inhibition of KF neurons reduces the respiratory response to central and peripheral chemoreceptor activation [63]. The KF contains laryngeal premotor neurons that control upper airway resistance [65] and spinally projecting neurons that control phrenic motoneuron activity [66]. Together, these studies suggest the pontine respiratory group as a key mediator in the basal respiration and the respiratory response to chemoreceptor activation.

\section{Retrotrapezoid Nucleus/parafacial respiratory group (RTN/pFRG)}

The RTN is a bilateral cluster of glutamatergic neurons located in the rostral medulla oblongata close to the ventral surface of the brainstem. It innervates portions of the ventral respiratory column as well as the KFN, the LBPN, and the NTS. Following activation, the RTN increases breathing [67]. A subset of RTN neurons expressing Phox2b are known to respond to $\mathrm{CO}_{2}$; indeed mice lacking Phox $2 \mathrm{~b}$ respond poorly to $\mathrm{CO}_{2}$ and 
suffer fatal central apneas at birth $[59,68]$. It has also been shown that even small lesions within the RTN can cause apnea [69]. Previous studies have also assessed the role of RTN neurons in breathing by utilizing viral vectors and the Phox $2 \mathrm{~b}$ promotor to acutely excite or inhibit the RTN. Optogenetic stimulation of these RTN neurons elicits an increase in respiratory rate and amplitude of breathing in adult rats. Inhibition of RTN neurons does the opposite [68]. Together, these studies point to the RTN as a key mediator in maintaining ventilatory drive and in the ventilatory response to $\mathrm{CO}_{2}$.

\section{pre-Bötzinger (pre-BötC) and Bötzinger (BötC) complexes}

Within the ventral respiratory column resides the pre-BötC, which is a bilateral region of glutamatergic interneurons that have a burst like firing pattern and operates as a rhythm generator for breathing [70]. Even in an isolated brainstem slice preparation, the pre-BötC continues to generate respiratory motor activity [71], which persists after disrupting synaptic inhibition [72]. The pre-BötC also contains glycinergic and GABAergic neurons, which provide phasic inspiratory inhibition in the brainstem, including inhibition of BötC expiratory neurons [73]. In vivo, disruption of the rhythmic activity in the pre-BötC elicits an irreversible loss or disruption in breathing [71].

Many studies have also utilized the presence of neurokinin-1 receptor immunoreactivity (NK1R-ir) as an approach for assessing chemoreceptor sites due to their high presence within these regions, providing an approach for cell specific lesions $[34,74]$. Indeed, this approach has been successful in evaluating the role of pre-BötC in the control of breathing in adult rats. In rats, loss of NK1R expressing neurons within the pre-BötC elicits an ataxic breathing pattern and a reduced ventilatory response to changes in inspired 
$\mathrm{CO}_{2}$ [34]. Together, these studies point to the pre-BötC as a key mediator in respiratory rhythm generation and in the ventilatory response to $\mathrm{CO}_{2}$.

The Bötzinger Complex (BötC) is caudal to the facial nucleus, mainly contains inhibitory glycinergic expiratory neurons [75], and is involved in the control of the transition between inspiratory and expiratory activities in the network. This feature is fundamental to the rhythmic inspiratory-expiratory alternation essential for normal breathing [76]. The BötC has extensive inhibitory connections with other respiratory neurons, including within other ventral respiratory column compartments. Further, reports suggest that BötC varicosities are seen in the RVLM. However, the main output of the BötC is towards respiratory neurons [77]. Further, the BötC projects to the cervical spinal cord, innervating the phrenic motor nucleus [78], and thus has the potential to control diaphragm activity (depth of breathing).

\section{5 - Overview of the sleep-wake cycle and arousal from sleep}

Understanding the normal mammalian sleep cycle allows for a unique understanding of sleep-to-wake transitions. Mammalian sleep is fairly predictable in a normal 24-hr day, with increased drowsiness and a desire to sleep towards the evening hours. This process is called the drive to sleep [79]. The suprachiasmatic nucleus (SCN) of the hypothalamus was identified as a critical brain structure that helps maintain the timing of the 24-hour circadian rhythm and drive to sleep in studies using rodents or non-human primates. This process is exemplified in humans: as daylight decreases and night approaches, there is an increased desire to sleep and drowsiness. However, the timing of this process is species-specific. Organisms sleep when the need for vigilance is minimized 
to reduce the risk of predation [79]. Loss of wakefulness and sleep consolidation occurs if there is a loss of input from the SCN [80], a feature that occurs in both nocturnal and diurnal species [81].

While progress in determining the molecular basis for oscillations in circadian rhythms have been made, the specific neural mechanisms are still unknown. The SCN may inhibit neural circuits that induce sleep, thereby promoting arousal during the active phase. Alternatively, the SCN may inhibit arousal circuits in the rest phase to promote sleep. However, the SCN may function to do both, depending on the period [82]. Identifying the neuronal processes involved in transitions between sleep states, as well as arousal from sleep, has been of interest to researchers for decades. Early work in the field led to the concept of an ascending reticular activating system (ARAS), consisting of dorsal and ventral pathways that modulate wake-promoting and sleep-promoting systems of the CNS [5]. A balance between these wake-promoting (arousal) and sleeppromoting regions in the brain exist to help initiate and sustain sleep or wakefulness. Anatomical and physiological techniques have allowed for the identification of these regions within the CNS [83].

The transition from wakefulness to non-rapid eye movement (NREM) sleep involves a balance between inhibition of wake-promoting areas and activation of sleeppromoting areas. Within the ventrolateral preoptic (VLPO) area, GABAergic neurons are thought to play a role in this transition from wakefulness to NREM. During wakefulness, the VLPO is suppressed by the wake-promoting monoaminergic and cholinergic neurons located in the arousal centers in the brainstem and diencephalon. During the transition period, the VLPO sleep-promoting neurons increase firing, causing an inhibition of wake- 
promoting neurons. Additionally, they also increase firing proportionally with increasing sleep depth. This process is mirrored by a progressive decrease in the firing rates of wakepromoting neurons in the brainstem-thalamic activating system [5, 84].

NREM to rapid-eye movement (REM) transitions are also thought to reciprocally cooperate with the cholinergic and monoaminergic populations in the brainstem as well as the glutamatergic neurons in the subcoeruleus (SubC) and GABAergic neurons of the ventrolateral periaqueductal gray (vlPAG). The monoaminergic REM-off neurons of the dorsal raphe nucleus (DRN) and LC cease firing in the transition from NREM to REM. The REM-on neurons of the two main cholinergic mesopontine nuclei then become disinhibited: the laterodorsal nuclei (LdT) and the pedunculopontine nucleus (PPT). Once the LdT/PPT neurons are activated/disinhibited, they in turn activate neurons within the reticular formation in a positive feedback style that generates REM sleep events. Transitioning between REM and NREM can occur, which involves the cessation of cholinergic REM-on neuron activity. The mechanism ultimately leads to a reciprocal interaction where the REM-off LC and DRN neurons inhibit the cholinergic REM-on neurons until they stop firing and REM ends [5, 84]. In REM sleep, experimental studies have shown that the glutamatergic population of neurons located ventral to the LC, the SubC, produces atonia and REM sleep-like EEG activity, likely mediated via its projections to the ventromedial medulla and ventral horn of the spinal cord. The SubC is also strongly inhibited by GABAergic REM sleep-suppressing neurons in the mid-pons vlPAG [85]. GABAergic neurons within the lateral pontine tegmentum (LPT) are also thought to inhibit REM sleep since lesioning of this region increases REM sleep in both mice and rats, similar to what occurs with lesioning of the vlPAG GABAergic population 
[5]. Melanin-concentrating hormone ( $\mathrm{MCH})$ neurons are also REM-on neurons and contain the neurotransmitter GABA. While these neurons innervate similar regions as the orexinergic neurons, they have an opposite activity profile - inhibiting the same targets during sleep that orexin neurons activate during wakefulness. However, the exact role of $\mathrm{MCH}$ neurons remains unclear since mice lacking MCH don't exhibit differences in REM sleep compared to WT counterparts [6]. More recently, a heterogeneous population of neurons located near the RVLM, called the lateral paragigantocellular nucleus (LPGi), was implicated in the control of sleep. This region contains neurons that are both REM-On and REM-Off. It has been postulated that the REM-On neurons of the LPGi are either GABAergic or glycinergic, but the neurotransmitter expressed has not been validated. Stimulation of the LPGi elicits an inhibitory response in a portion of LC neurons, supporting the idea that at least some of the LPGi contains an inhibitory neurotransmitter [86]. REM-On LPGi neurons are perfectly situated to mediate some of the sleep-state dependent changes in cardiorespiratory control. Anterograde labeling indicates that LPGi neurons project to the NA, where preganglionic CVNs are located. Indeed, activation of the LPGi with orexin elevates HR, which is at least partially mediated via inhibition of PSNS activity to the heart [86]. A summary schematic of these regions is in Fig. 1.3. 


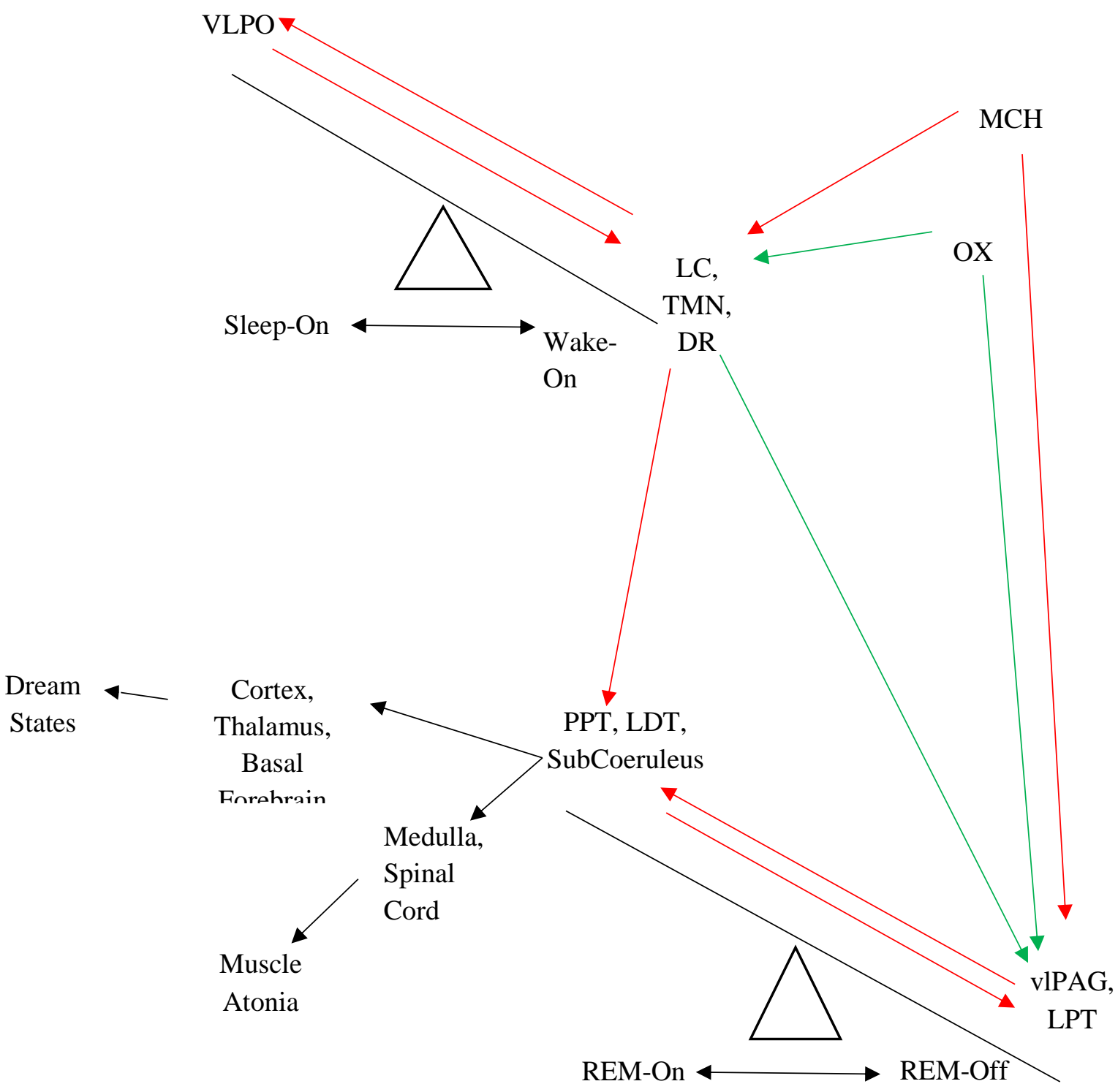

Fig. 1.3: Summary schematic of wake and sleep-promoting neurons shown as components of a "switch". REM-on and REM-off populations are located in the lower right. Green arrows are excitatory and red arrows are inhibitory. The monoaminergic neurons (locus coeruleus, LC; tuberomammillary nucleus, TMN; dorsal raphe, DR) inhibit the ventrolateral preoptic (VLPO) nuclei and the REM-on neurons (laterodorsal nuclei (LdT) and pedunculopontine nucleus (PPT)) and excite the REM-off neurons (lateral pontine tegmentum (LPT) and ventrolateral periaqueductal gray (vlPAG)) during wakefulness - making it unlikely for normal individuals to transition from wakefulness directly into REM sleep. Orexinergic (OX) and melaninconcentrating hormone $(\mathrm{MCH})$ neurons project to the same regions, but have opposing actions (e.g. the OX neurons maintain wakefulness by exciting monoaminergic and REM-off neurons). As an example of how this model works, during REM sleep, when the balance of REM-off and REM-on neurons tips towards REM-on neurons, there is greater activation of regions like the PPT/LDT and less activation of monoaminergic regions in the brainstem, producing typical PGO waves of REM via projections to the cortex/forebrain. Further, during REM sleep, the SubCoeruleus contributes to muscle atonia. Adapted from [6] 


\section{6 - Cardiorespiratory function during sleep}

Autonomic control of cardiorespiratory function is modulated differently in each of the sleep-states and depends on the balance of activity between sleep- and wake-promoting nuclei, many of which project to key areas involved in cardiorespiratory control. In both animals and humans, PSNS activity is highest in NREM, as evidenced by an elevated high frequency component of heart rate variability, which is thought to reflect PSNS tone to the heart. Relative to wakefulness and REM sleep, HR and ABP is lower and is more stable in NREM[87, 88]. However, compared to wakefulness and NREM, there are distinct fluctuations in cardiorespiratory rhythms and core body temperature during REM [79]. Overall, $\mathrm{ABP}, \mathrm{HR}$, and respiratory rate (RR) increase in REM compared to NREM. However, all three variables are lower in NREM and REM when compared to wakefulness [89]. In addition to a reduced ventilation during NREM sleep, metabolic rate also falls [5]. Further, the reduced ventilation is associated with a larger tidal volume $\left(\mathrm{V}_{\mathrm{T}}\right)$ and slower $\mathrm{RR}$, accompanied by a relative increase in $\mathrm{PaCO}_{2}$ and concomitant decrease in $\mathrm{PaO}_{2}$ [90]. Respiration is profoundly irregular during REM, associated with sudden changes in $\mathrm{V}_{\mathrm{T}}$ and $\mathrm{RR}$, which varies by species. $\mathrm{PaCO}_{2}$ decreases, approaching awake values, while $\mathrm{PaO}_{2}$ remains the same or is slightly elevated compared to NREM [93]. These changes in respiration are accompanied by PSNS withdrawal [94].

Respiratory stimuli, including changes in $\mathrm{PaO}_{2}, \mathrm{PaCO}_{2}$, and respiratory mechanoreceptor activity can induce arousal [95]. Hypercapnia increases ventilation, ABP and SNA [96]. Both peripheral and central chemoreceptors are thought to play a role in the arousal process, especially as it relates to diseases like sleep apnea. Activation of central chemoreceptors (hypercapnia) or selective activation of the carotid bodies (hypoxia) is 
sufficient to cause arousal, including in humans [97]. In dogs, both hypoxia and airway occlusion cause arousal; removal of the carotid bodies attenuates this effect, suggesting that the carotid bodies contribute to hypoxia-induced arousal $[98,99]$. Additionally, the arousal response to hypercapnia, hypoxia, and upper airway stimulation was attenuated in dogs during REM [100]. Records also indicate that increased $\mathrm{CO}_{2}$ can cause arousal, but requires seemingly high levels of $\mathrm{CO}_{2}$ to do so. Inspired $\mathrm{CO}_{2}$ levels of $<5 \%$ substantially increased ventilation but did not affect arousal [101]. The $\mathrm{CO}_{2}$ arousal threshold varies between individuals, species, and on the method used to induce hypercapnia. Additionally, the combination of hypercapnia and hypoxemia is a more potent stimulus to arousal than hypoxia alone [102].

Mechanoreceptors in or near the respiratory tract may also play a role in the arousal process. The respiratory muscles, chest wall, and upper and lower airways are potential sites where mechanoreceptors may contribute to the arousal stimulus during increased respiratory effort. However, the mechanoreceptor contribution to the arousal stimulus remains unclear [102]. For instance, studies in patients with chronic neurologically complete spinal cord injuries above $\mathrm{C} 3$, thus lacking central connections from chest wall mechanoreceptors, have shown that hypercapnia can consistently induce arousal from sleep. Hypercapnia induced arousal in these patients was most likely caused by central mechanisms such as changes in respiratory drive projecting to arousal neurons or direct effects of $\mathrm{CO}_{2}$ on arousal nuclei [95]. If the mechanoreceptors do contribute to the arousal stimulus, their importance likely depends on location as well as receptor properties and levels of respiratory effort reached prior to arousal [102]. 
Several brainstem and forebrain systems are involved in mediating sleep and wakefulness, including the arousal process. These sleep/wake regulatory systems include GABAergic nuclei of the VLPO, 5-HT, Ach, histamine, noradrenaline, and orexin/hypocretin. Here, I will briefly discuss their roles in sleep/arousal and potential influences on cardiorespiratory control during sleep. A diagram of their projections with respect to other sleep/wake systems is pictured in Fig. 1.3. Their effect on cortical activity, as measured by EEG, as well as eye movements and muscle tone are summarized in Table 1.1.

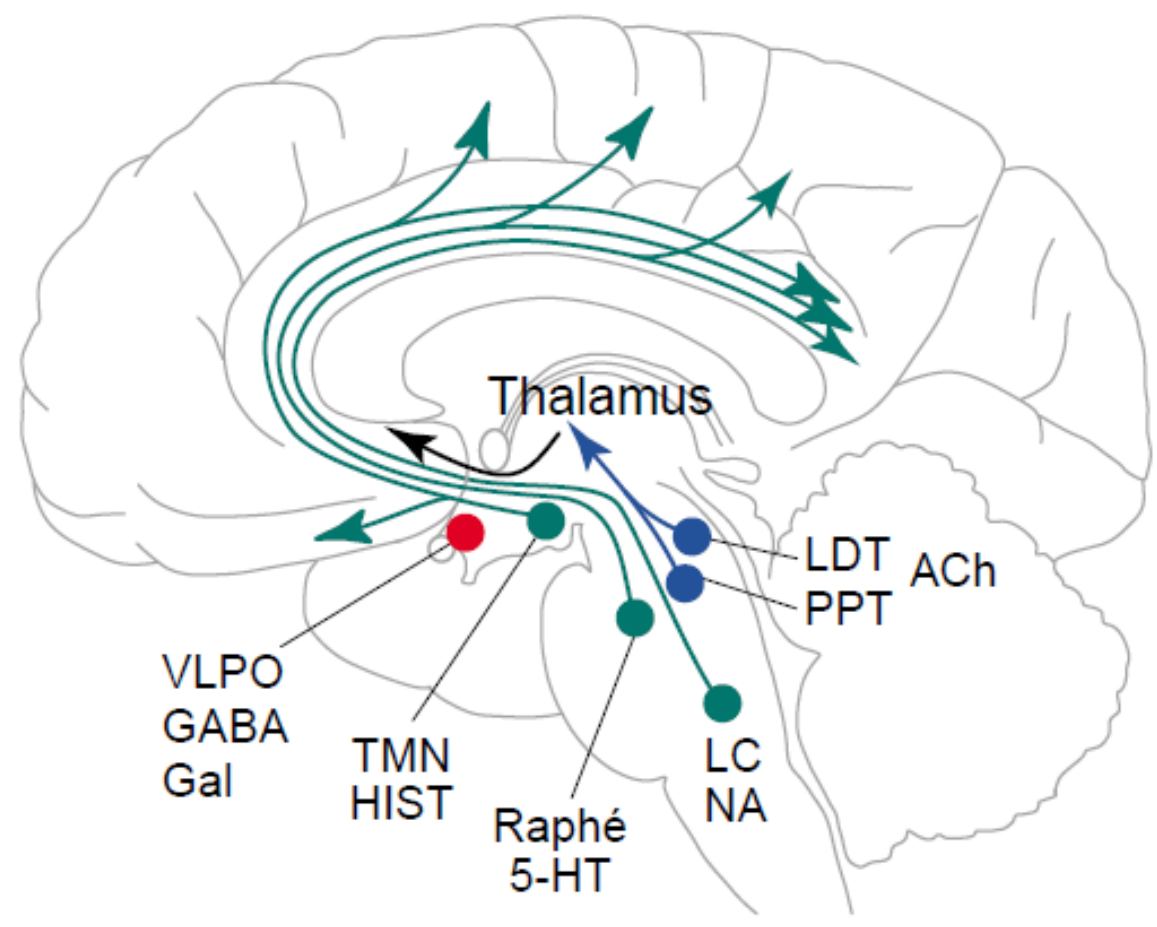

Fig 1.3: The ascending arousal system (ARAS) sends projections from the posterior hypothalamus and brainstem throughout the forebrain. Neurons of the pedunculopontine tegmental nuclei and laterodorsal tegmental nuclei (PPT and LDT, blue) send cholinergic (Ach) projections to many forebrain targets to regulate cortical activity. Monoaminergic nuclei (green) regulate the activity of cortical and hypothalamic targets directly. The tuberomammillary nucleus contains histaminergic neurons, the raphe nuclei contain serotonergic neurons and the locus coeruleus contains noradrenergic (NA) neurons. Sleep promoting neurons of the ventrolateral preoptic nucleus (VLPO, red) contain GABA and galanin (Gal). [3] 


\begin{tabular}{llll} 
& Wakefulness & NREM sleep & REM sleep \\
\hline EEG & Fast, low voltage & Slow, high voltage & Fast, low voltage \\
Eye movement & Vision related & Slow, infrequent & Rapid \\
Muscle tone & $\uparrow \uparrow$ & $\uparrow$ & 0 \\
LDT/PPT & $\uparrow$ & 0 & $\uparrow \uparrow$ \\
LC/DR/TMN & $\uparrow \uparrow$ & $\uparrow$ & 0 \\
VLPO cluster & 0 & $\uparrow \uparrow$ & $\uparrow ?$ \\
VLPO extended & 0 & $\uparrow ?$ & $\uparrow \uparrow$ \\
Orexin/hyprocretin & $\uparrow \uparrow$ & $0 ?$ & $0 ?$
\end{tabular}

Table 1.1 This table summarizes electroencephalography (EEG) measurements as well as relative intensity of eye movements during each of the sleep states. It also summarizes the firing rates as follows: two arrows $=$ rapid firing, one arrow $=$ slower firing, $0=$ little or no firing. Question marks represent a hypothesized firing pattern from the literature where there is not firm evidence. LdT/PPT = laterodorsal and pedunculopontine tegmenti (acetylcholine); LC/DR/TMN: locus coeruleus/dorsal raphe/tuberomammillary nucleus (noradrenaline/serotonin/histamine); VLPO: ventrolateral preoptic nucleus (GABA). [3]

\section{$\boldsymbol{G A B A}$}

$\gamma$-aminobutyric acid (GABA) neurons within the VLPO are the main inhibitory neurons within the CNS modulating sleep-state. Reports have demonstrated that these neurons discharge at a higher rate during sleep than wakefulness. Additionally, GABAergic neurons within the VLPO display an increased c-Fos expression during sleep, consistent with their role in sleep modulation. It has been proposed that these neurons project widely; innervating several neuronal groups including the serotonergic neurons, histaminergic neurons of the tuberomammillary nucleus (TMN), noradrenergic LC neurons, and orexinergic neurons. Thus, these neurons are well situated to play a role in sleep/wake modulation and arousal. In support of their role during sleep, studies have shown that extracellular levels of GABA are increased during sleep and pharmacological experiments have reported that $\mathrm{GABA}_{\mathrm{A}}$ receptor activity enhances NREM in adults (quiet sleep in pups) [80]. Many studies have implicated a role for GABA in cardiorespiratory control, through 
GABAergic neurons and receptors on key sites involved in the regulation of respiration and ABP. A subpopulation of VLPO neurons project to the PVN, which projects to sympathetic preganglionic neurons of the spinal cord and presympathetic motor neurons in the RVLM [103]. The VLPO neurons have been implicated in decreasing SNA during NREM via its inhibitory projection to PVN sympathoexcitatory neurons [104]. Despite the vast projections of these GABAergic VLPO neurons, direct evidence for their role in cardiorespiratory control during sleep is lacking.

\section{Acetylcholine (Ach)}

Cholinergic neurons within the LdT/PPT provide inputs the thalamus and to arousal and attention-promoting regions of the hypothalamus, including the orexin neurons of the lateral hypothalamus and histaminergic neurons of the TMN [5]. Ach has also been proposed to influence sleep/wake cycle through the basal forebrain (BF). The BF is a major source of cholinergic projections to the cortex and BF cholinergic neurons exert an excitatory influence on cortical targets. They also play an important role in behavior, arousal and EEG desynchronization. During both wakefulness and REM sleep, the BF cholinergic neurons have a high firing rate and Ach release into the cortex is high. During NREM in adults (quiet sleep in pups), these neurons are relatively quiet and cortex Ach levels are low [105]. This demonstrates the BF nuclei as an important wake-promoting region.

\section{Histamine}


Histaminergic nuclei, located in the TMN, project to most regions of the CNS, many of which contain one or more of the histamine receptors $\left(\mathrm{H}_{1}, \mathrm{H}_{2}\right.$, and $\left.\mathrm{H}_{3}\right)$ [106] and are implicated in the sleep/wake cycle. Targeted neurons include 5-HT neurons within the medullary raphe, orexin $(\mathrm{OX})$ neurons of the hypothalamus, noradrenergic neurons within the locus coeruleus, and cholinergic neurons of the mesopontine tegmentum and BF [107]. Similar to other wake-promoting nuclei, these neurons are active during wakefulness and cease firing during NREM in adults (quiet sleep in pups) and REM in adults (active sleep in pups) $[80,106]$. c-Fos studies in rats have also shown that histaminergic neurons are quiescent during the light or inactive phase of the circadian light cycle [108]. Similar to 5HT neurons, the histaminergic neurons are thought to be inhibited during sleep via GABAergic innervation from the VLPO, a region that shows high activity during NREM $[106,109]$. Several reports also suggest that histamine is required for wakefulness. Blockade of histamine has been shown to promote sleep [110]; indeed, antagonizing $\mathrm{H}_{1}$ receptors increases NREM in adult rodents and mice lacking histamine show a deficit in waking and attention [106].

Histamine neurons project to key cardiorespiratory nuclei, like the NTS, PVN and RVLM, implicating a role for histamine in cardiorespiratory homeostasis. Immunohistochemical studies have shown that rats exposed to $5 \% \mathrm{CO}_{2}$ exhibited an increase in c-Fos expression within the TMN. Thus, there may be a role for central histamine in modulating respiration [106]. Exogenously applied $\mathrm{H}_{1}$ and $\mathrm{H}_{3}$ agonists increase phrenic nerve frequency in the working-heart-brainstem-preparation. However, only $\mathrm{H}_{1}$ antagonists reduce respiratory frequency. These results suggest that the excitatory effects of histamine are likely through $\mathrm{H}_{1}$ receptors [111]. Additionally, the effects of 
histamine on cardiovascular control may be region specific. Local administration of histamine into the $\mathrm{PVN}$ increases $\mathrm{ABP}$ and $\mathrm{HR}$, a response likely through $\mathrm{H}_{1}$-receptors as administration of an $\mathrm{H}_{1}$-receptor antagonist abolished these effects. Plasma catecholamines are also increased after histamine injections into the PVN, indicating an increase in sympathetic drive. However, bilateral administration of histamine into the RVLM of anesthetized rats elicits hypotension and bradycardia, which is blocked by pretreatment with an $\mathrm{H}_{2}$-receptor antagonist. Additionally, activation of $\mathrm{H}_{1}$-receptors within the NTS elicits increases in both ABP and HR [112]. Thus, the role of histamine may vary depending on the receptor, and on whether anesthesia is present. In summary, histaminergic nuclei are well situated to play a role in cardiorespiratory control and the sleep/wake cycle.

\section{Noradrenaline Neurons of the Locus Coeruleus (LC-NA)}

The LC is the largest noradrenergic nucleus in the brain and projects to almost all brain regions. This includes projections to the hypothalamus, NTS, the DMX, and NA, to name a few [114]. Thus, these neurons are well situated to play a role in sleep/wake cycles and cardiorespiratory control.

Optogenetic studies have shed light on the role of LC-NA neurons in the arousal process. In one report, optogenetic stimulation of the LC-NA caused arousal from both REM and NREM [115]. The LC has reciprocal projections to several of the other wakepromoting regions, including the OX neurons in the hypothalamus, 5-HT neurons in the medullary raphe and Ach neurons in the LdT/PPT [5]. In one optogenetic study, photostimulation of the LC-NA intensified the effects of OX stimulation on wakefulness 
while photoinhibition of the LC-NA blocked the effects of OX stimulation. These results suggest that the LC-NA is a downstream effector of OX induced arousal [116].

Depletion of the Phox2a gene, which is responsible for differentiation of catecholaminergic neurons restricted to the LC proper, selectively blocks the LC-NA neurons from forming but leaves other noradrenergic centers intact. Phox $2 a^{-/-}$mice have been linked to severe decreases in breathing frequency. This finding implicates the LC-NA neurons in providing a tonic excitatory stimulus to maintain breathing frequency in addition to being necessary for the development of a normal respiratory rhythm [117]. LCNA neurons also appear to play a role in the in vivo HCVR during sleep. Lesioning of the LC-NA with 6-hydroxydopamine (6-OHDA) reduces NA neurons by approximately $84 \%$ and elicits a significant reduction in the response to $7 \% \mathrm{CO}_{2}$ by approximately $28 \%$ in sleep and wakefulness [117]. Taken together, these studies implicate the LC-NA neurons in the control of sleep/wakefulness, respiration, and chemoreception.

\section{$\operatorname{Orexin}(O X)$}

OX neurons have been implicated in a variety of processes, including sleep/wake cycles, arousal, cardiorespiratory control, and the chemoreflex [96]. OX neurons discharge during both wakefulness and just before waking but virtually cease firing during NREM and REM. However, some reports indicate that the firing rate of OX neurons is slightly higher in REM than NREM [118] and there is elevated orexin in the cerebrospinal fluid in REM compared to NREM [119]. Despite their low firing rate during sleep, OX neurons retain the capacity to sustain neural activity in the absence of synaptic input [120]. Furthermore, OX concentration varies almost two-fold during the circadian cycle in 
rodents, monkeys, and humans, being highest at the end of the dark/active period and lowest towards to end of the light/inactive period [121].

Despite an unclear mechanism of how OX neurons promote sleep-to-wake transitions, a role for $\mathrm{OX}$ in maintaining/promoting wakefulness is evident from the literature. Activation of $\mathrm{OX}_{1}$ receptors has been shown to promote wakefulness and suppress NREM; however, activation of $\mathrm{OX}_{2}$ receptors elicits the same results with higher efficacy. In addition, there is profound dysregulation of REM sleep control from a loss of signaling through both $\mathrm{OX}_{1}$ and $\mathrm{OX}_{2}$ receptors [122].

Several reports explain orexin's participation in the regulation of respiration and the hypercapnic ventilatory response (HCVR). Adult prepro-orexin knockout mice, which are devoid of OX, have normal basal ventilation during wakefulness, NREM and REM [123]. However, these mice have a significantly attenuated HCVR during wakefulness but not during NREM or REM [124]. Supplementation with OX in these mice partially restores the HCVR [125]. Despite a reduced HCVR, the knockout mice maintained both $\mathrm{PaO}_{2}$ and $\mathrm{PaCO}_{2}$ during the $5 \% \mathrm{CO}_{2}$ challenge [124] suggesting ventilation is matched to metabolic rate. $\mathrm{OX}$ is also implicated in the regulation of cardiovascular function. Intracerebroventricular administration of $\mathrm{OX}$ in conscious rats has been shown to increase ABP and HR [126]. Additionally, ABP and HR increases in both anesthetized and awake rats when OX is microinjected into the RVLM or NTS [127, 128]. Prepro-orexin knockout mice have been shown to exhibit a lower ABP at rest compared to controls, by about 20mmHg [129]. In the spontaneously hypertensive rats (SHR), antagonizing OX not only lowers $\mathrm{ABP}$, but reduces the elevated CSF and plasma noradrenaline and adrenaline 
detected in the model $[96,130]$. Taken together, these studies suggest a role for OX in the regulation of sleep/wakefulness, cardiorespiratory control and the chemoreflex.

\section{Serotonin (5-Hydroxytryptamine, 5-HT)}

Dorsal raphe (DRN) 5-HT neurons (rostral/superior group) are active during wakefulness and quiet sleep/NREM (QS in pups, NREM in adult rodents) but are virtually quiescent in active sleep/REM (AS in pups, REM in adult rodents) [5]. Furthermore, the activity of this rostral/superior group of 5-HT neurons has an inverse relationship with the occurrence of the sleep spindles and ponto-geniculo-occipital cortex (PGO) waves that are associated with NREM and REM sleep, respectively. This relationship does not hold for the medullary (caudal/inferior) 5-HT neurons. Medullary 5-HT neurons (raphe obscurus (NRO), raphe pallidus (NRPa) and raphe magnus (NRM)) also have a higher firing rate (by 30-60\%) than 5-HT neurons in the DRN during comparable vigilance-states [131]. Furthermore, the NRO/NRPa do not display as great of a decline in activity across the sleep-wake cycle, although they are still significantly suppressed during REM sleep compared to QW and NREM [131]. Although some reports suggest that the medullary raphe neurons have a similar firing pattern as DRN 5-HT neurons, being highest in wakefulness and absent during REM sleep [4]. Development does not appear to play a significant role in the characteristic firing patterns of DRN neurons; indeed, DRN 5-HT neurons have a slow and regular activity within the first postnatal week of the cat and an adult-like pattern of activity across sleep-wake cycles [131].

5-HT metabolism is influenced by the light-dark cycle. However, the activity of the DRN 5-HT neurons appears to be constant irrespective of phase of the light-dark cycle. It 
has been suggested that differences in 5-HT metabolism during the light-dark cycle may be attributed to differences in the gross activity of the animals. For instance, rats typically spend the majority of the dark cycle awake and active; thus they would have an increased 5-HT metabolism compared to the light cycle, in which rats are mostly sleeping [131].

5-HT neurons project to several regions involved in the control of sleep and wakefulness, including the VLPO, the histaminergic neurons within the TMN, cholinergic neurons within the LdT/PPT, and basal forebrain GABAergic neurons projecting to the hippocampus. 5-HT also plays a role in sleep-state architecture, i.e. the duration or frequency of QS/NREM or AS/REM [5, 132, 133]. Indeed, microinjection of 5-HT into the LdT of freely behaving adult rats suppresses REM sleep [132].

Furthermore, several of the 5-HT receptors have been associated with 5-HT's role in sleep, in particular, the 5- $\mathrm{HT}_{1 \mathrm{~A}}, 5-\mathrm{HT}_{1 \mathrm{~B}}, 5-\mathrm{HT}_{2 \mathrm{~A}}$ and $5-\mathrm{HT}_{7}$ receptors. Indeed, activation of 5- $\mathrm{HT}_{1 \mathrm{~A}}$ receptors in the medullary raphe nearly eliminates REM sleep in piglets [134]. Similarly, 5-HT $T_{1 B} /$ - mice have been shown to spend a significantly greater amount of time in REM sleep [135]. Similarly, activation of the 5- $\mathrm{HT}_{1 \mathrm{~B}}$ receptor has been shown to reduce REM sleep in rats [136] suggesting that 5-HT activation of 5-HT 1 B receptors reduces REM sleep similar to activation of $5-\mathrm{HT}_{1 \mathrm{~A}}$ receptors. Several reports indicate a role for $5-\mathrm{HT}_{7}$ receptors in sleep; for instance, 5-HT7 knockout mice spend less time in REM during the light phase comparted to WT mice [137]. Furthermore, infusion of SB-269970, a potent 5$\mathrm{HT}_{7}$ receptor antagonist, elicits a reduction in REM sleep [138]. Taken together, these findings suggest that 5-HT promotes wakefulness and inhibits REM sleep.

5-HT and 5-HT neurons also appear to be important in arousal from sleep, which has implications in both obstructive sleep apnea and sudden infant death syndrome (SIDS). 
For instance, mice with a genetic lesion of 5-HT neurons $\left(L m x 1 b^{f f f p}\right)$ do not arouse from sleep following a hypercapnic stimulus [139], mediated at least in part by the loss of 5$\mathrm{HT}_{2 \mathrm{~A}}$ receptor activation [140]. However, these mice do arouse to other stimuli like hypoxia or auditory stimuli [141]. Similarly, neonatal rat pups lacking tryptophan hydroxylase 2 (TPH2), the rate limiting enzyme in the synthesis of central 5-HT, have a delayed arousal to hypercapnia but not hypoxia [142], suggesting that 5-HT promotes arousal during hypercapnic conditions, as occurs during apnea.

The interactions of these neuromodulators with respect to each other is shown in Fig. 1.4. 


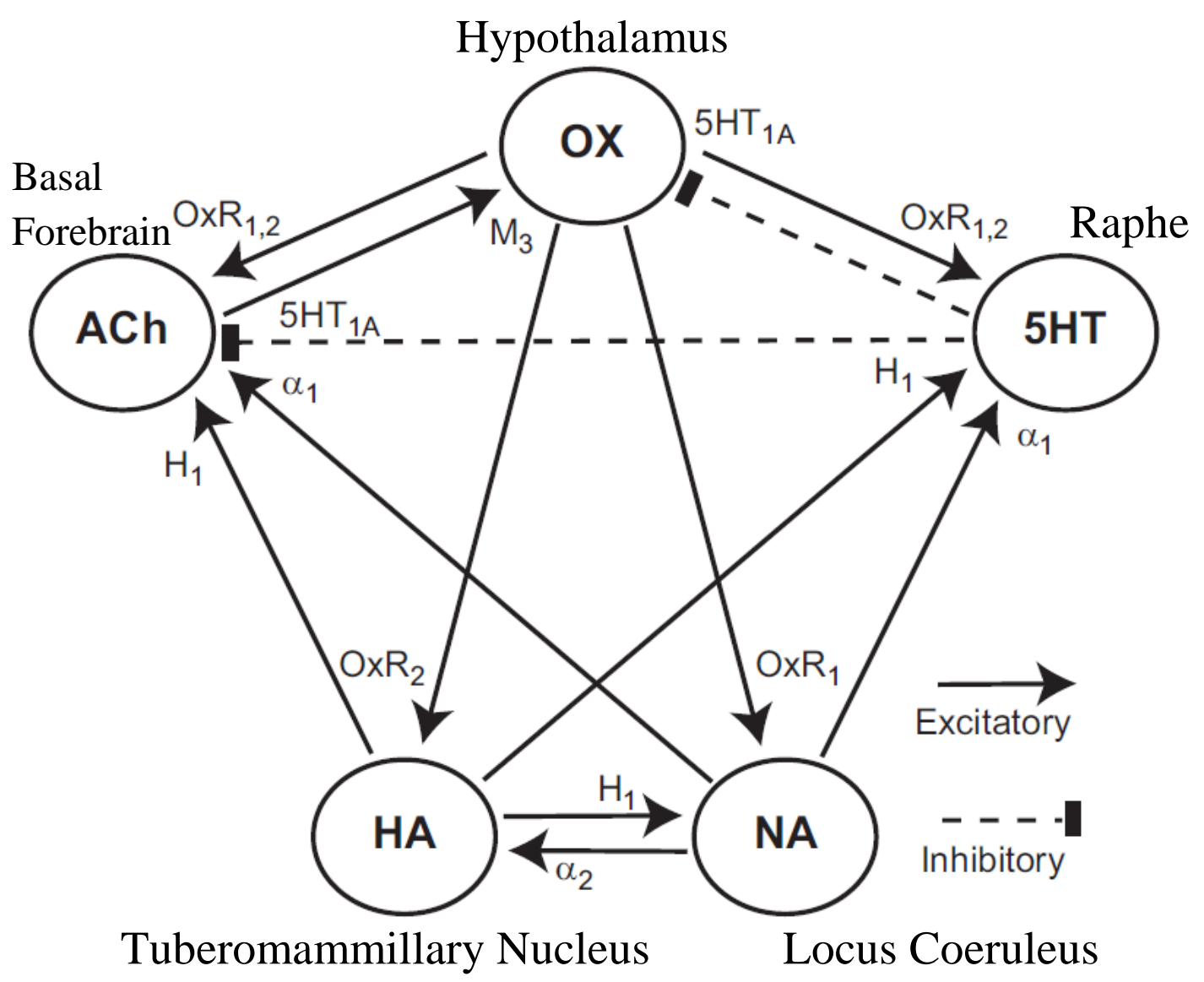

Fig. 1.4: Summary of the functional interactions between wake promoting neurotransmitters that project to the forebrain. Cholinergic (Ach) neurons of the basal forebrain (BF), orexinergic (OX) lateral hypothalamic, serotonergic (5-HT) raphe, noradrenergic (NA) locus coeruleus, and histaminergic (HA) tuberomammillary neurons interact to promote wakefulness. GABAergic mechanisms also play a role in sleep regulation and are described in the text. Even with lesioning of one region, other systems maintain the ability to control cortical activation/wakefulness. Receptors involved in this process include: $\alpha 1$, alpha-adrenergic type 1; $\alpha 2$, alpha-adrenergic type 2; H1, histaminergic type 1; M3, muscarinic cholinergic; OxR1, orexin receptor type 1; OxR2, orexin receptor type $2 ; 5-\mathrm{HT}_{1 \mathrm{~A}}$, serotonergic type $1 \mathrm{~A}$. From [5] 


\section{$\underline{\text { Serotonin }}$}

\section{Biosynthesis and Catabolism}

5-HT is a highly conserved and phylogenetically ancient biogenic amine [143] located in both the central (CNS) and peripheral (PNS) nervous system of mammals. Originally identified as enteramine, 5-HT plays a role as early as fetal life in morphogenesis and neural trafficking in addition to physiological processes like eating, reward, thermoregulation, locomotion, pain, memory, cognition, cardiorespiratory homeostasis, and the sleep/wake cycle to name a few [1].

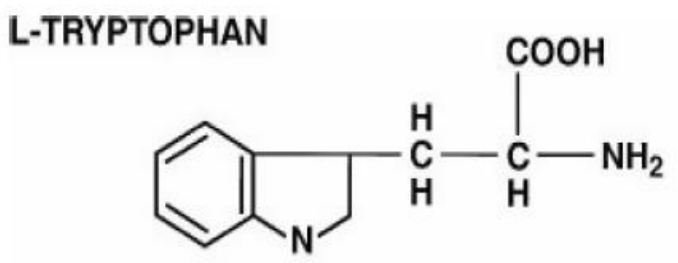<smiles>NC(C=CC1CNc2ccc(O)cc21)C(=O)O</smiles>

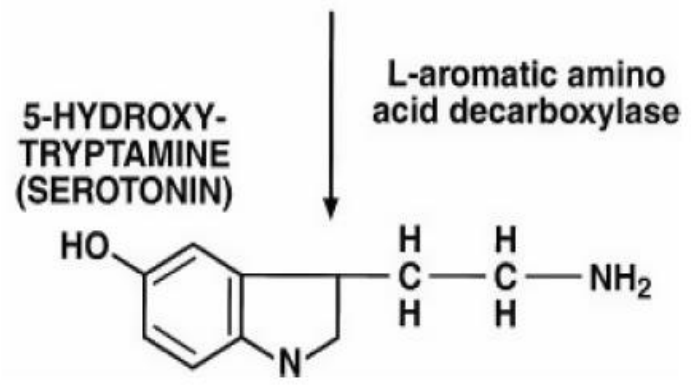

5-HT is synthesized from the amino acid tryptophan in two steps by

Fig 1.5: Biosynthesis and catabolism of 5$\mathrm{HT}$; in the pineal gland, 5-HT is converted to melatonin [1].

tryptophan hydroxylase (TPH) and amino acid decarboxylase (Fig 1.5). TPH is the rate limiting step in the synthesis of 5-HT, of which there are two forms, TPH1 and TPH2. TPH1 synthesizes peripheral 5-HT, whereas TPH2 synthesizes central 5-HT [144]. TPH can be inactivated by methylenedioxymethamphetamine (MDMA) and pchloroamphetamine, which elicits extensive damage to 5-HT neurons $[145,146]$. The mechanism is unknown but thought to be due to drug-induced production of reactive 
oxygen species (ROS) and/or nitric oxide (NO). At least with NO, inactivation of TPH is by selective attack on critical sulfhydryl groups [147].

In the CNS, 5-HT neurons are located within the raphe nuclei of the brainstem [131]. 5-HT neurons consist of a superior (rostral) and inferior (caudal) group of midline raphe neurons that were originally divided into nine clusters of cell bodies (B1-B9) [131]. The DRN consists of groups B6 and B7 while the median raphe (MRN) consists of groups B5 and B8. The inferior (caudal) group consists of 5-HT neurons within the raphe obscurus (NRO, B2), pallidus (NRPa, B1 and B4), and magnus (NRM, B3) as well as the ventral lateral medulla and area postrema. These inferior/caudal 5-HT neurons predominately influence cardiorespiratory control, including upper airway control, autoresuscitation (a sequence of respiratory and cardiovascular responses that reoxygenate the sinoatrial node to restore $\mathrm{HR}, \mathrm{ABP}$, and eventually normal breathing [148]), central chemoreceptor responses to hypercapnia, and cardiovascular control $[131,143]$. The dense projections to the IML are predominately through the NRO and NRPa, which also project to the NTS, NA, DMX, and brainstem respiratory groups $[4,131]$. Retrograde labeling has even shown dense fiber networks containing 5-HT surrounding cell bodies of the phrenic motor nucleus [149]. A depiction of serotonergic projections in relation to cardiovascular control are shown in Fig. 1.6. 5-HT neurons also express many other neurotransmitters, including substance P, thyrotropin releasing hormone (TRH), enkephalin, and GABA [150, 151]. Quantitative studies in the rat indicate that a large number of 5-HT neurons within the area postrema also contain norepinephrine [131].

5-HT fibers terminate in various regions throughout the brain, and participates in thermoregulation [152], sleep [153], aggression [154], and cardiorespiratory control [155- 
158]. This includes a dense serotonergic input to the sympathetic preganglionic neurons located in the intermediolateral nucleus (IML) of the spinal cord [159]. 5-HT neurons also receive inputs from several brainstem and forebrain areas involved in motor control, autonomic regulation, and pain modulation, including the periaqueductal gray,

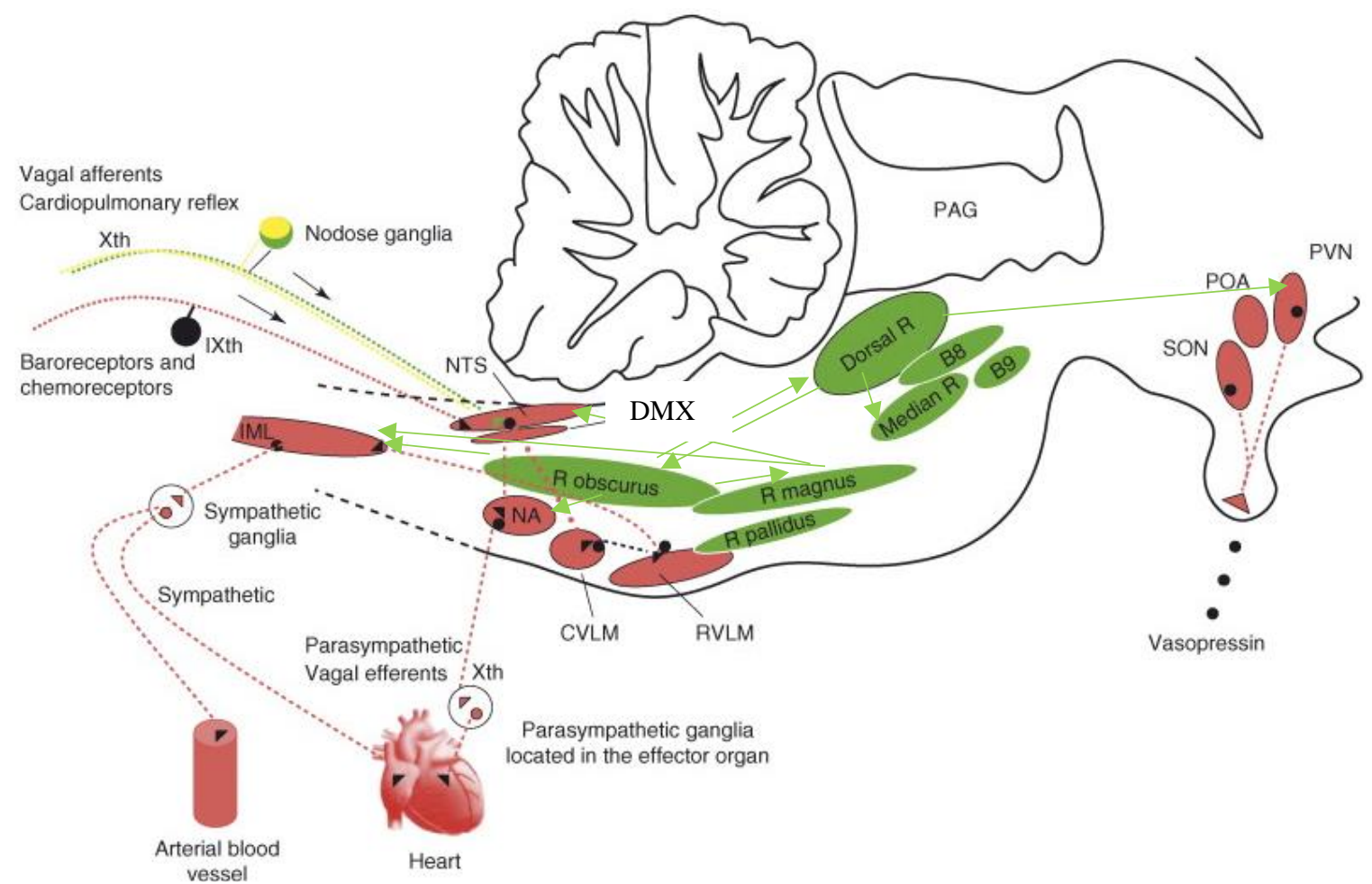

Fig. 1.6: Schematic diagram showing 5-HT containing neuronal groups (green) and projections (green arrows) in relation to central cardiovascular regions (red) in the brainstem and hypothalamus. Dotted lines show connections between cardiovascular regions involved in the baroreflex; dotted red lines = excitatory; dotted black lines $=$ inhibitory; dotted yellow $=$ glutamatergic vagal afferents $(X$ th cranial nerve); dotted green $=$ potential serotonergic containing afferents. Baroreceptor/Chemoreceptor afferents feed into the NTS, relaying information on pressure, $\mathrm{PO}_{2}, \mathrm{pH}$, etc; traveling mainly via the glossopharyngeal nerve (IXth cranial nerve) with cardiopulmonary afferents travelling via the vagus. Following integration at the NTS, information is sent to the RVLM (sympathetic) and nucleus ambiguus/dorsal vagal nucleus (PSNS tone to the heart). The baroreceptor pathway also includes a pathway from the NTS to the CVLM, which supplies tonic inhibitory (GABAergic) drive to premotor sympathetic neurons in the RVLM that innervate the preganglionic sympathetic neurons in the IML. Hypothalamic nuclei are also shown (e.g. the supraoptic nucleus and PVN) which are involved in the release of vasopressin and receive cardiovascular information from the NTS. The PVN sends efferents to the IML, the RVLM and the raphe nuclei as well as to the HPA axis. The B1-B4 5-HT groups overlap with some of the ventral surface cardiovascular areas and send projections caudally, some innervating the IML. The dorsal raphe (B6 and 7), median raphe (B5 and B8) and nucleus pontis oralis (B9) send projections rostrally, innervating cardiovascular areas in the hypothalamus in addition to the most of the forebrain. Modified from [8] and [10] 
parabrachial nucleus, the amygdala, hypothalamus and catecholaminergic pontomedullary groups [4]. This includes both glutamatergic [160] and GABAergic input [161]. At least in the DRN, one of the likely sources of the GABAergic tone is the LPGi [162].

Central 5-HT has also been shown to alter the release of other regulators of cardiovascular control, including AVP and renin. For instance, intracerebroventricular (i.c.v.) injection of 5-HT results in the release of AVP [163], which has a well-known role in cardiovascular control [164]. Similarly, lesioning of the dorsal raphe inhibits the effect stress has on plasma renin concentration [165]. However, plasma renin release is potentiated following a foot-shock stressor in rats with a lesion in the MRN [166]. These data suggest a potential relationship between the central 5-HT system and the reninangiotensin system.

Central 5-HT can also modify glutamate and GABA mediated effects, acting through one of the 14 functionally distinct receptors. Through modulating glutamate or GABA, 5-HT can potentially contribute to cardiorespiratory control and sleep. For instance, within the PVN, GABA and glutamate are major neurotransmitters where the vast majority of local synaptic inputs are GABAergic. 5-HT has been shown to inhibit GABA release from pre-sympathetic $\mathrm{PVN}$ neurons via $5-\mathrm{HT}_{1 \mathrm{~A}}$ receptors [167]. 5-HT can also modulate glutamatergic inputs to cardiac vagal neurons; following hypoxia/hypercapnia, 5-HT activates 5- $\mathrm{HT}_{1 \mathrm{~A}}$ receptors and reduces the activity of cardiac vagal neurons (CVNs) residing in the NA and DMX through inhibition of glutamatergic input [168]. 5-HT also promotes wakefulness, through depolarization of basal forebrain (BF) GABAergic neurons that project to the hippocampus. Further, 5-HT also suppresses NREM sleep, likely through a 5- $\mathrm{HT}_{1 \mathrm{~A}}$ receptor-mediated postsynaptic inhibition of the sleep-active GABAergic VLPO 
neurons [5]. In summary, 5-HT can modulate GABAergic and glutamatergic drive in the brain, modulating physiological functions including cardiovascular control and the control of sleep/wakefulness.

Within 5-HT neurons, there is evidence to suggest that 5-HT synthesis, calcium dependent exocytosis, reuptake, and metabolism operates in all neuronal elements (i.e. soma, dendrites, axons, and terminals) [12, 169]. Further, the concentration of 5-HT within the CNS is modulated by a variety of factors including negative feedback of 5-HT via binding to somato-dendritic 5-HT $\mathrm{HA}_{1 \mathrm{~A}}$ autoreceptors [170] as well as removal of 5-HT from the presynaptic membrane by the highly selective 5-HT transporter (5-HTT). 5-HTT is a member of the $\mathrm{Na}+\mathrm{Cl}-$ transporter family, which also includes dopamine, norepinephrine, GABA and glutamate transporters [1]. 5-HTT displays an affinity for 5-HT in the nanomolar range [171]. When 5-HT is recycled into presynaptic vesicles via 5-HTT, it is protected from metabolism, which only occurs in the cytoplasm through MAO [1]. Clearance of 5-HT is in the 100-200 millisecond range and is thought to reflect 5-HTT activity in vivo [172], where 5-HTT activity is highest within the DRN [173]. The alternative pathway for 5-HT is located in the pineal gland, where 5-HT converts to melatonin [1]. A depiction of the handling of 5-HT at the synapse is shown in Fig. 1.7. 


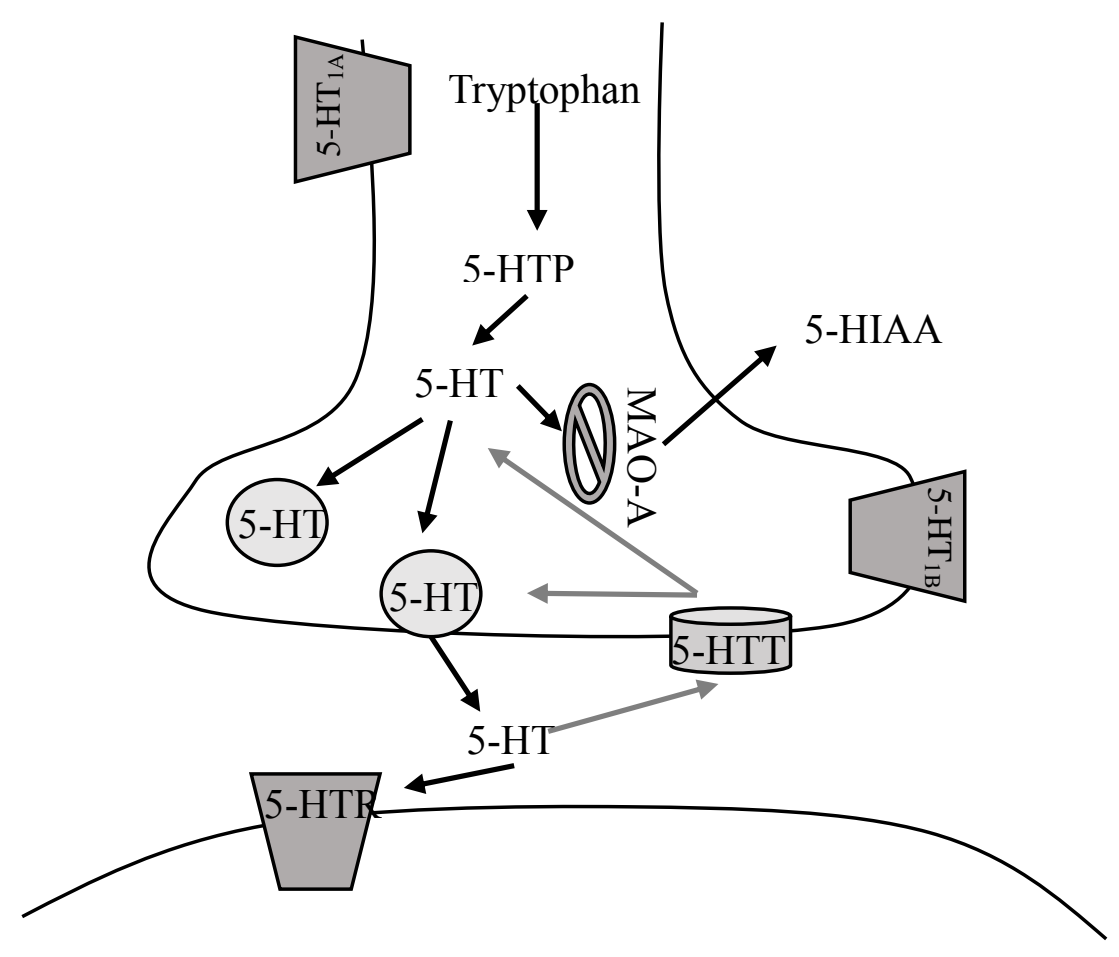

Fig 1.7: Depiction of synaptic handling of 5-HT from synthesis, storage, release, and uptake via 5-HTT and metabolism. Somatodendritic 5- $\mathrm{HT}_{1 \mathrm{~A}}$ receptors can also contribute to neuronal firing and some reports suggest that $5-\mathrm{HT}_{1 \mathrm{~B}}[4]$ receptors can inhibit neurotransmitter release at terminals (see text for description). Adapted from [9]

Evidence also indicates contribution of other factors in 5-HT clearance away from its release site. While 5-HTT's are primarily responsible for 5-HT clearance, the norepinephrine transporter (NET) has also been shown to clear locally applied 5-HT in the dentate gyrus [172]. This likely occurs similarly in other brain regions, although this has yet to be established. Mimicking studies based on the ability of the dopamine (D2) autoreceptor to inhibit the dopamine transporter, it has also been shown that the $5-\mathrm{HT}_{1 \mathrm{~B}}$ autoreceptor, $\alpha 2$-adrenoreceptors, and the adenosine receptor can regulate 5-HTT activity [173]. 
In the periphery, 5-HT is extensively synthesized in the enterochromaffin cells of the gastrointestinal tract, transported to platelets via the 5-HT transporter (5-HTT) and stored in platelets through the action of the vesicular monoamine transporter VMAT2 [144, 174]. 5-HT's concentration is dependent on its rate of synthesis, metabolism, and clearance. Monoamine oxidase (MAO) is the primary enzyme for the metabolism of 5-HT, which exists in two forms: MAO-A and MAO-B. 5-HT is primarily inactivated by MAOA into 5-hydroxyindoleacetic acid (5-HIAA). MAO-B is located in human platelets and represents a small portion of 5-HT metabolism. 5-HIAA is excreted in urine rapidly metabolized by MAO - within 24 hours of 5-HT administration, the majority of 5-HIAA is excreted [1]. Presumably both peripherally and centrally derived 5-HIAA is excreted through urine as more than $75 \%$ of central 5-HIAA can be removed directly from the brain to blood via an active transport system [175].

\section{Serotonergic Receptors}

The 5-HT system has seven families of receptors with at least 14 receptors that are genetically, pharmacologically, and functionally distinct. These receptors are located on pre- and/or post-synaptic cell membranes [176]. Most of the 5-HT receptors belong to the G-protein-coupled receptor (GPCR) family with the exception of the 5- $\mathrm{HT}_{3}$ receptor, which is a ligand-gated ion channel [177]. As 5-HT acts mainly through G-protein coupled receptors, post-synaptic responses are slower than through ligand-gated ion channels.

5-HT receptors have diverse function within the central nervous system, with a variety of physiological outputs based on the specific receptor activated. Most 5-HT receptors are associated with specific physiological responses ranging from modulation of 
neuronal activity and neurotransmitter release to behavioral modifications (e.g. 5-HT abnormalities contribute to the development of depression [178]). Within the CNS, 5-HT neurons within the NRO and NRPa innervate autonomic areas in the brainstem and spinal cord to mediate serotoninergic regulation of the cardiovascular and respiratory systems, predominately-through 5- $\mathrm{HT}_{1 \mathrm{~A}}$ and 5- $\mathrm{HT}_{2 \mathrm{~A}}$ receptor subtypes, which are heavily concentrated within the RVLM [144].

\section{5-HT 1 Receptors}

The 5-HT $1 \mathrm{~A}$ receptor was the first to be fully sequenced, identified by screening a genomic library for homologous sequences to the $\beta 2$-adrenoreceptor [179]. 5-HT $\mathrm{HT}_{1 \mathrm{~A}}$ activation of Gi subunits results in the inhibition of adenylate cyclase and a subsequent decrease in cAMP $[180,181]$. 5- $\mathrm{HT}_{1 \mathrm{~A}}$ receptors can act as both autoreceptors (acting on 5-HT neurons) and as heteroceptors (acting post-synaptically). Within the raphe nuclei, 5$\mathrm{HT}_{1 \mathrm{~A}}$ receptors act as autoreceptors to inhibit 5-HT neuronal firing via their location on the soma and dendrites of 5-HT neurons [177]. Activation of 5-HT $1 \mathrm{~A}$ autoreceptors induces a fall in 5-HT release in the rat forebrain in vivo [179]. Post-synaptic 5- $\mathrm{HT}_{1 \mathrm{~A}}$ receptors are distributed throughout the CNS and are coupled to inhibitory G-proteins. Thus, activation of these receptors causes post-synaptic hyperpolarization, through G-protein coupled $\mathrm{K}^{+}$ channels [177]. However, in the raphe, these receptors reduce calcium currents and evoked calcium influx [183].

As previously mentioned, 5- $\mathrm{HT}_{1 \mathrm{~A}}$ heteroceptors are involved in the regulation of sleep. For instance, activation of 5- $\mathrm{HT}_{1 \mathrm{~A}}$ receptors in the medullary raphe nearly eliminates REM sleep in piglets [134]. 5-HT $1 \mathrm{~A}$ heteroceptors are also located in regions involved in 
the control of $\mathrm{HR}$ and $\mathrm{ABP}$. In adult rats, 5-HT binding to 5- $\mathrm{HT}_{1 \mathrm{~A}}$ receptors reduces the activity of cardiac vagal neurons (CVNs) residing in the NA and DMX through inhibition of glutamatergic input [168]. The primary site of 5-HT $1 \mathrm{~A}$ mediated sympatho-inhibition is the RVLM [8]. Indeed, microinjection of 8-OH-DPAT, a 5-HT 1 A agonist, into the RVLM induces a fall in ABP and HR that can be blocked by 5-HT $1 \mathrm{~A}$ antagonists [163].

5- $\mathrm{HT}_{1 \mathrm{~A}}$ receptors are also implicated in the control of breathing during sleep. In addition to reducing REM sleep, 5- $\mathrm{HT}_{1 \mathrm{~A}}$ receptor activation with 8-OH-DPAT in the PPT of adult rats increases both respiratory and genioglossus activity [133]. Furthermore, neonatal $5-H T_{1 A} /-$ mice have reduced ventilation at rest, compared to WT littermates [184]. 5-HT $1 \mathrm{~A}$ receptors are also implicated in the central chemoresponse. Indeed, inhibition of raphe magnus 5-HT neurons with 8-OH-DPAT, a 5-HT 1 A receptor agonist, decreases the $\mathrm{CO}_{2}$ response [69]. Furthermore, inhibition of 5-HT neurons by focal microdialysis of 8-OH-DPAT into the medullary raphe attenuates the ventilatory response to hypercapnia in piglets older than 8 days [185].

5-HT $\mathrm{HB}_{1 \mathrm{~B}}$ receptors are also located both in 5-HT terminals (autoreceptors) as well as in non-5-HT terminals (heteroceptors), where they inhibit neurotransmitter release [4]. 5$\mathrm{HT}_{1 \mathrm{~B}}$ receptors are most prevalent in human cerebral arteries and has been implicated in the pathogenesis of migraine [181]. 5-HT $1 \mathrm{~B}$ receptors are linked to adenylate cyclase and are involved in the regulation of the synaptic release of 5-HT as well as Ach, NE, GABA and glutamate, exemplifying its role as both an auto- as well as hetero-ceptor [186]. As previously mentioned, 5- $\mathrm{HT}_{1 \mathrm{~B}}$ receptors are involved in the regulation of sleep. Similar to activation of 5- $\mathrm{HT}_{1 \mathrm{~A}}$ receptors, activation of 5- $\mathrm{HT}_{1 \mathrm{~B}}$ receptors on the ventral surface of the 
cat medulla elicits a fall in ABP and HR [187]. Taken together, these studies support a role for $5-\mathrm{HT}_{1 \mathrm{~A}}$ and $5-\mathrm{HT}_{1 \mathrm{~B}}$ receptors in cardiorespiratory control and in sleep/wake regulation.

\section{5-HT 2 Receptors}

Unlike 5- $\mathrm{HT}_{1 \mathrm{~A}}$ receptors, $5-\mathrm{HT}_{2}$ receptors primarily exert a post-synaptic excitatory effect through coupling to $\mathrm{G}_{\mathrm{q} / 11}$, activating phospholipase $\mathrm{C}$, and producing inositol triphosphate 3 (IP3) and diacylglycerol (DAG). The activation of IP3 stimulates $\mathrm{Ca}^{2+}$ release from the endoplasmic reticulum while DAG activates protein kinase $\mathrm{C}$ to inhibit $\mathrm{K}^{+}$channels [179].

5- $\mathrm{HT}_{2}$ receptors have long been implicated in the control of ABP. Indeed, similar to $5-\mathrm{HT}_{1 \mathrm{~A}}$ receptors, the major site of action of 5-HT2 receptors is the RVLM. However, NTS neurons can also be activated by $5-\mathrm{HT}_{2}$ receptors [8]. Activation of NTS 5-HT2 receptors by 2,5-Dimethoxy-4-iodoamphetamine (DOI) produces the typical responses of baroreceptor activation - hypotension and bradycardia [188, 189]. This suggests that 5$\mathrm{HT}_{2}$ receptors in the NTS play a facilitatory role in the reflex regulation of ABP. I am unaware of whether 5-HT interacts with signaling downstream of 5-HT. However, 5-HT has been shown to act at regions like the RVLM. For instance, microinjection of DOI into the subretrofacial nucleus of the RVLM increases ABP [163], suggesting that activation of 5- $\mathrm{HT}_{2 \mathrm{~A}}$ receptors within the RVLM are mediating at least part of this response. On the other hand, blockade of 5- $\mathrm{HT}_{2}$ receptors in the CNS does not lower ABP, but it does prevent the development of deoxycorticosterone acetate (DOCA)-salt hypertension. The mechanism is likely through $5-\mathrm{HT}_{2 \mathrm{~A}}$ mediated activation of a central angiotensinergic pathway eliciting the release of AVP, a component essential for the development of 
DOCA-salt hypertension [8], where blocking 5- $\mathrm{HT}_{2 \mathrm{~A}}$ receptors prevents the development of DOCA-salt hypertension. Activation of forebrain 5- $\mathrm{HT}_{2}$ receptors are also implicated in the sympathoexcitatory effects of 5-HT. Sympathetic nerve activity, ABP, and HR increase in adult rats treated with DOI in the lateral ventricle [190].

5- $\mathrm{HT}_{2}$ receptors also play a role in the control of breathing. For instance, activation of hypoglossal motoneurons by $5-\mathrm{HT}$ is mimicked by the $5-\mathrm{HT}_{2}$ agonist DOI. Furthermore, 5-HT is known to stimulate the $\mathrm{RTN}$, a major site for central $\mathrm{CO}_{2}$ chemoreception, an effect that is mostly 5- $\mathrm{HT}_{2 \mathrm{~A}}$ dependent [157]. As will be discussed in more detail later, the 5-HT dependent long-term increase in phrenic activity following intermittent hypoxia is dependent on the $5-\mathrm{HT}_{2}$ receptor $[191,192]$.

\section{5-HT Receptors $_{3}$}

$5-\mathrm{HT}_{3}$ receptors are unique, in that among the 5-HT receptors, it is the only ligandgated ion channel. These receptors trigger a rapid depolarization due to a transient inward current, following the opening of nonselective cation channels $\left(\mathrm{Ca}^{++}\right.$influx, $\mathrm{K}^{+}$efflux, $\left.\mathrm{Na}^{+}\right)$ [177]. Centrally, the NTS contains the highest density of 5- $\mathrm{HT}_{3}$ receptors, most of which

are located on vagal afferent terminals $[8,193]$; however, diffuse staining for $5-\mathrm{HT}_{3}$ receptors has been observed in forebrain regions like the hippocampus, amygdala and, to a lesser extent, the reticular and paraventricular thalamic nuclei [194]. Activation of 5-HT3 receptors within the NTS elicits a relatively consistent excitatory response, despite 5-HT within the NTS having a variety of effects including excitation, inhibition and biphasic responses to activation of other 5-HT receptor types (i.e. 5- $\mathrm{HT}_{1 \mathrm{~A}}$ and $5-\mathrm{HT}_{2}$ ) [61]. Within the NTS, agonism of 5- $\mathrm{HT}_{3}$ receptors has been shown to inhibit the cardiac component of 
the baroreflex and the Bezold-Jarisch reflex, without affecting their sympathetic components [50-52]. In vivo, application of either 5-HT or the selective 5- $\mathrm{HT}_{3}$ receptor agonist 1-phenylbiguanide (PBG), increases the activity of the DMX, an effect that could be attenuated by 5-HT3 antagonism [195], suggesting that 5- $\mathrm{HT}_{3}$ receptors reduce HR.

Widespread evidence implicates the $5-\mathrm{HT}_{1 \mathrm{~A}}$ and $5-\mathrm{HT}_{2}$ receptors in regulating sleep but less is known about the role of central 5- $\mathrm{HT}_{3}$ receptors in sleep/wakefulness. Agonism of 5- $\mathrm{HT}_{3}$ receptors has been shown to reduce REM sleep whereas antagonism of 5- $\mathrm{HT}_{3}$ receptors prevented the effects of 5- $\mathrm{HT}_{3}$ agonism on REM sleep [196]. Overall, these reports suggest that $5-\mathrm{HT}_{3}$ receptors may play a role in the cardiovascular control and sleep/wakefulness.

\section{5-HT 4 Receptors}

5- $\mathrm{HT}_{4}$ receptors are one of the three 5-HT receptors (in addition to 5- $\mathrm{HT}_{6}$ and 5$\mathrm{HT}_{7}$ ) positively coupled to heterotrimeric Gs proteins, exerting its excitatory effects through the activation of adenylyl cyclase. This process increases intracellular cAMP levels to induce an increase in cellular excitability. Several lines of evidence suggest a role for 5- $\mathrm{HT}_{4}$ receptors in mood, cognition, and learning [197], but to my knowledge, a role for $5-\mathrm{HT}_{4}$ receptors in the sleep/wake cycle has not been determined.

5- $\mathrm{HT}_{4}$ receptors are abundantly expressed in the pre-BötC, co-localized in a subset of NK1 receptor positive neurons; stimulating 5- $\mathrm{HT}_{4}$ receptors increases respiratory activity [198]. Peripheral 5- $\mathrm{HT}_{4}$ receptors are thought to play a role in cardiovascular regulation. For instance, 5-HT increases atrial function and arrhythmias as well as positive inotropic effects at the ventricle, primarily through the 5-HT4 receptor [199]. However, 
few studies have addressed the role of central $5-\mathrm{HT}_{4}$ mediated actions on cardiovascular control. Reports suggest that activation of $5-\mathrm{HT}_{4}$ receptors in the NTS attenuate the cardiopulmonary reflex induced bradycardia and tachypnea [200]. However, future experiments are necessary to assess the physiological relevance of this $5-\mathrm{HT}_{4}$ mediated depression of the cardiopulmonary reflex, including the mechanisms that can activate 5$\mathrm{HT}_{4}$ receptors within the NTS (peripheral afferent and/or central projections) [200]. These results suggest that $5-\mathrm{HT}_{4}$ receptors may play a role in cardiovascular and respiratory control; the extent to which $5-\mathrm{HT}_{4}$ receptors contribute to sleep regulation remains to be elucidated.

\section{5-HT5 Receptors}

5-HT5 receptors have long been considered an "orphan" receptor, in that little is known about them. The transductional mechanisms associated with $5-\mathrm{HT}_{5}$ receptors in native tissue are still in question, although in vitro studies suggest that it does not couple with either cAMP or $\mathrm{IP}_{3}$ pathways [201], while others suggest it couples to Gi/o signaling pathways $[181,202]$. It has been suggested that $5-\mathrm{HT}_{5}$ receptors reduce sympathetic drive, but considering the absence of specific agonists and antagonists, it is difficult to discern the specific role of 5- $\mathrm{HT}_{5}$ receptors in cardiorespiratory control [203]. Overall, research is needed to assess the role of 5-HT5 receptors in cardiorespiratory control and sleep/wake regulation.

\section{5-HT6 Receptors}


The 5- $\mathrm{HT}_{6}$ receptor is one of the last 5-HT receptors to be discovered and is one of three serotonin receptors positively coupled to the Gs protein, inducing cAMP production [177]. Its functional role in the brain is ill-defined. However, the 5-HT6 receptor is most abundant in forebrain regions like the frontal cortex, implicating it as a modulator of cognition and mood [204]. To my knowledge, well-defined roles for 5-HT 6 receptors in cardiorespiratory control and in the regulation of sleep/wakefulness are yet to be assessed. However, recent reports do suggest that blockade of $5-\mathrm{HT}_{6}$ receptors increases wakefulness and reduces NREM and REM sleep [205], which may be related to inhibition of neurotransmitter systems crucial for NREM and REM sleep (i.e. the preoptic area or the LdT/PPT).

\section{5-HT 7 Receptors}

The 5- $\mathrm{HT}_{7}$ receptor is one of three serotonin receptors positively couple to the Gs protein, inducing cAMP production [177]. 5- $\mathrm{HT}_{7}$ receptors have garnered attention due to its moderate affinity for 8-OH-DPAT, a 5-HT agonist thought to be specific to $5-\mathrm{HT}_{1 \mathrm{~A}}$ receptors [206]. 5- $\mathrm{HT}_{7}$ receptors have been implicated in the modulation of cardiovascular reflexes. For instance, antagonism of $5-\mathrm{HT}_{7}$ receptors attenuates the cardiovascular responses to chemo-, baro-, and cardiopulmonary reflexes [53]. 5- $\mathrm{HT}_{7}$ receptors also appear to play a role in the plasticity that followings intermittent hypoxia; episodic activation of the $5-\mathrm{HT}_{7}$ receptor in spinal motor neurons elicits phrenic motor facilitation in anesthetized rats [207]. The 5-HT 7 receptor has also been implicated in the sleep/wake cycle. For instance, 5-HT 7 knockout mice spend less time in REM. Further, microinjection of 5- $\mathrm{HT}_{7}$ receptors in the $\mathrm{DRN}$ reduces REM sleep. It was proposed that this is mediated 
by activation of GABAergic neurons in the DRN that project to the LdT/PPT to elicit the reduction in REM sleep [208]. In summary, 5- $\mathrm{HT}_{7}$ receptors appear to play a role in cardiovascular reflexes, plasticity following intermittent hypoxia, and in the sleep/wake cycle.

\section{The role of 5-HT in cardiorespiratory control at rest}

Several pharmacological studies suggest a functional role for central 5-HT in the control of $\mathrm{HR}$ and $\mathrm{ABP}$. In adult rats, $5-\mathrm{HT}$ binding to $5-\mathrm{HT}_{1 \mathrm{~A}}$ receptors reduces the activity of cardiac vagal neurons (CVNs) residing in the NA and DMX, increasing HR. This is due to 5- $\mathrm{HT}_{1 \mathrm{~A}}$ mediated inhibition of glutamatergic input, and thus the overall balance of glutamatergic and GABAergic tone CVNs is shifted towards GABA, eliciting a reduction in PSNS activity. This may be relevant in SIDS infants, who may have reduced 5-HT signaling, and could therefore have an overall increase in PSNS activity through this pathway [168], possibly reducing HR. Further studies utilizing adult rats show that 5-HT3 receptor activation within the commissural NTS elicits hypertension [209]. In addition, cardiac sympathetic nerve activity, $\mathrm{HR}$ and $\mathrm{ABP}$ increase in adult rats treated i.c.v. with DOI [190]. However, adult rats systemically treated with para-chlorophenylalanine ( $p$ CPA), a brain permeant 5-HT synthesis inhibitor that depletes CNS and peripheral 5-HT content, had a significant rise in baseline ABP [210]. Thus, the overall effect of central 5-HT on HR and ABP is unclear, and has not been investigated in the neonatal period. Infant rats and mice deficient in 5-HT neurons also demonstrate cardiovascular abnormalities that could put them at risk for sudden death. Pet-1-/- mice with a genetically induced deficiency of 5-HT neurons exhibit reduced resting HR [158]. In addition, we have 
recently shown that neonatal rats treated centrally with 5,7-dihydroxytryptamine $(5,7-$ DHT, a neurotoxin selective to 5-HT neurons) exhibit a reduced resting ABP and HR compared to saline injected controls [156]. These pups also have more severe apneic induced bradycardias [211]. Given that 5-HT neurons contain other neuromodulators like substance $P$, thyrotropin releasing hormone and even glutamate [157], how a specific loss of 5-HT signaling contributes to these phenotypes is unclear.

In adult animals, there is a lack of consensus regarding the effect of central 5-HT on the strength of the baroreflex. Following drug-induced depletion of central 5-HT content, adult rats have been shown to be hypertensive; with reduced gain of the phenylephrine (PE) and sodium nitroprusside (SNP) evoked cardiac baroreflexes [210]. However, rats in which 5-HT neuronal projections are lesioned specifically in the NTS display an augmented SNP-induced reflex tachycardia [212].

Infant mice and rats deficient in 5-HT neurons also demonstrate respiratory abnormalities similar to those of SIDS cases. In the neonatal period, mice deficient in $\operatorname{Lmx} 1 \mathrm{~b}\left(\operatorname{Lmx} 1 b^{f f / p}\right)$, which selectively lack 5-HT neurons, have frequent and severe apneas. These apneas can be as long as 55s in length and are associated with a marked decrease in ventilation [213]. However, 5-HT neurons contain other neuromodulators like substance $\mathrm{P}$, thyrotropin releasing hormone and even glutamate [157]. Recent data in neonatal rats lacking TPH2 suggest that a specific loss of 5-HT also plays a role in respiratory function. Like $L m x 1 b^{f f / p}$ mice, as neonates, TPH2-/- rats hypoventilate at rest and have frequent and severe apneas [155]. Interestingly, most of the apneas demonstrated by these animals occur during active (REM) sleep [214]. 
5-HT neurons are also putative chemoreceptors [215]. In studies utilizing the protein c-Fos, 5-HT neurons exhibited an increase in c-Fos expression after hypercapnic loading. This finding is consistent with in vitro studies showing that neurons within the raphe exhibit $\mathrm{CO}_{2}$ dependent changes in membrane potential and firing rate [110]. Adult mice with a near complete absence of 5-HT neurons $\left(L m x 1 b^{f f f / p}\right)$ have a blunted hypercapnic ventilatory response (HCVR), with a normal baseline ventilation [216]. Pet-1 knockout mice, which lack $\sim 60-80 \%$ of brainstem 5-HT neurons, also display respiratory dysfunction. These mice have a normal resting ventilation but a reduced ventilatory response to hypercapnia (both $7 \%$ and $10 \% \mathrm{CO}_{2}$ ) [217]. Additionally, inhibition of raphe magnus 5-HT neurons with 8-OH-DPAT, a 5- $\mathrm{HT}_{1 \mathrm{~A}}$ receptor agonist, decreases the $\mathrm{CO}_{2}$ response [69]. 5-HT may be important for an appropriate ventilatory response to hypoxia (HVR). Indeed, the HVR has been recently shown to be blunted in adult mice lacking TPH2 [218]. Taken together, these data suggest that 5-HT neurons are important in chemoresponses to hypercapnia and hypoxia.

5-HT is involved in the ABP and HR responses to severe hypoxia in neonatal rodents. For example, neonatal rodents treated with 6-fluorotryptophan (6-FL, a competitive inhibitor of tryptophan hydroxylase) exhibit a premature deterioration of $\mathrm{ABP}$ and HR following successive episodes of severe hypoxia [148]. Additionally, neonatal mice lacking TPH2 have a delayed HR recovery following severe hypoxia [219]. These data suggest that 5-HT is involved in the acute ABP and HR responses to severe hypoxia and adds to previous reports that failure of autoresuscitation may not be completely attributable to a failure of the gasping mechanism but due to impaired cardiac function [220]. These finding may be particularly relevant to SIDS cases, who die following one of 
likely many episodes of hypoxia [221] and up to $70 \%$ of SIDS cases have one or more abnormalities in the 5-HT system [222].

\section{The role of 5-HT in cardiorespiratory control during sleep}

As mentioned previously, 5-HT neurons project not only to regions involved in cardiorespiratory homeostasis but also to regions involved in sleep/wakefulness. Coupled with the state-dependency of their firing patterns [223], 5-HT neurons are anatomically well positioned to play a role in cardiorespiratory control during sleep, yet few studies have addressed this possibility. Telemetric monitoring demonstrates that adult $\mathrm{TPH} 2-/-$ mice have reduced $\mathrm{ABP}$ and $\mathrm{HR}$ at night [224]. However, sleep state was not monitored in this study, nor is it clear how a specific loss of 5-HT content affects the control of ABP and HR during sleep in infancy. Research in our lab has recently shown that TPH2-/- pups have reduced ventilation and $\mathrm{HR}$ during all sleep states (QW, QS, and AS). These pups also exhibit prolonged episodes of AS and a profound increase in the apneic index during AS compared to WT controls [225]. Two of my aims are directed towards elucidating the role of central 5-HT modulation of ABP and HR during sleep in neonatal and adult rodents.

\section{Serotonin and the plasticity that follows intermittent hypoxia}

Infants that die of SIDS have one of likely many episodes of hypoxia [221]. Following repetitive episodes of hypoxia (intermittent hypoxia (IH)), a long lasting (>1Hr) increase in respiration, phrenic, and sympathetic activity occurs. This plasticity is collectively termed long-term facilitation (LTF - respiratory, rLTF; phrenic, pLTF; sympathetic, sLTF). This phenomenon is only present when hypoxia is intermittent in 
nature and is generally considered a protective mechanism [34, 226]. There is evidence that both pLTF and sLTF are 5-HT dependent [227]. However, few studies have assessed how a specific loss of 5-HT alters the magnitude of LTF and the extent to which 5-HT is required for plasticity in neonatal life. Vigilance state may also play a role in vLTF. Recent work has demonstrated that vLTF exhibits profound vigilance state dependence in freelybehaving rats, with the greatest response in NREM sleep [228]. Whether this is true of sLTF is unknown.

Various experimental paradigms evaluate the mechanisms associated with rises in phrenic and sympathetic nerve activities following IH. The most extensively studied is the plasticity in phrenic motor output that follows IH. pLTF is typically studied in anesthetized, paralyzed, and ventilated rats with a standard IH protocol of three 5-min challenges of hypoxia. Following this IH protocol, the long-term increase in phrenic activity is dependent on the 5- $\mathrm{HT}_{2}$ receptor. $5-\mathrm{HT}_{2}$ receptor activation leads to synthesis of brain-derived neurotrophic factor (BDNF) which activates the high affinity tyrosine kinase B (TrkB) receptor to strengthen glutamatergic signaling on the phrenic motoneuron. This pathway is collectively coined the "Q" pathway, because it follows activation of a Gq protein. Moderate IH activates the Gq pathway. Severe IH activates the "S" pathway through Gs protein coupled receptors, like adenosine- $2 \mathrm{~A}$ and $5-\mathrm{HT}_{7}$. This pathway is $\mathrm{BDNF}$ independent through activation of immature intracellular TrkB receptors for enhancement of glutamatergic signaling on the phrenic motoneuron $[207,229,230]$. How the shift between the Q and S pathways occurs with severe IH is unknown but crosstalk inhibition between the pathways has been proposed [191, 192]. While sLTF is known to be 5-HT dependent like pLTF, the exact cellular mechanisms are currently unknown [227]. 
Recent work has shown that adult mice lacking TPH2 have ventilatory LTF, but the magnitude is significantly reduced compared to control littermates [218]. Previous reports have also assessed if this plasticity is present during neonatal life. Indeed, it has been shown that neonatal rodents exposed to episodic hypoxia exhibit an increase in genioglossus muscle activity [231] as well as rLTF [232]. Whether central 5-HT is required for pLTF in neonatal rodents is unknown.

\section{$\underline{1.8 \text { - Clinical Relevance }}$}

We seek to address the extent to which a specific loss of 5-HT affects the autonomic control of $\mathrm{HR}$ and $\mathrm{ABP}$ across vigilance-states based on existing evidence suggesting that 5-HT defects increase the risk for SIDS and neurogenic hypertension. SIDS occurs during sleep and SIDS cases also experience severe bradycardia and hypotension immediately before death, suggesting defects in the autonomic control of HR and ABP [221]. Neonatal rodents lacking 5-HT experience bradycardia and hypotension, resembling SIDS cases [156]. 5-HT has also been postulated as one of the mechanisms by which neurogenic hypertension manifests [233]. However, the mechanisms are poorly understood. Reports suggest that serotonergic over-activity in some brainstem nuclei contribute to the development of spontaneous hypertension [234] and blockade of serotonin production with 5,6-dihydroxytryptamine (5,6-DHT) blunts the development of spontaneous hypertension [235]. The goal of my project was to determine the mechanisms behind altered ABP in neonatal and adult rodents in the face of central 5-HT deficiency in an effort to tease out possible mechanisms in the development of these etiologies. 


\section{Sudden Infant Death Syndrome}

Physiological changes in cardiovascular and respiratory control occur during sleep. The prevalence of sleep-related breathing disorders is increasing. Sudden Infant Death Syndrome (SIDS), the leading cause of infant mortality between 1 month and one year of age, occurs during sleep [236, 237]. Infants that die of SIDS have dramatic and rapid changes in normal autonomic and respiratory patterns. Rare recordings from SIDS cases show that these infants experienced episodic tachycardia and bradycardia for hours to days prior to death, as well as overt bradycardia and hypotension immediately before death. These observations strongly suggest that a failure of autonomic mechanisms governing HR and MAP underlies SIDS [221].

Following the recognition that prone (facedown) sleeping was associated with an increased incidence of SIDS, consideration turned to factors that might trigger infant death in this position. This includes factors like asphyxia due to rebreathing of exhaled gases or airway compression due to sleeping in the prone position in addition to a compromised arousal in response to asphyxia when sleeping facedown. Drs. Filano and Kinney proposed the Triple Risk Factor model that encapsulates what occurs in infants that die of SIDS [221]. All three factors must occur for SIDS to occur, including: 1) an underlying vulnerability in the infant (i.e. in brainstem neurotransmitter systems); 2) a critical period of development (2-6 months of age); and 3) an exogenous stressor (e.g. excessive heat or hypoxia) [221]. In addition to this Triple Risk Factor Model, there are additional risk factors associated with an increased incidence of SIDS. This includes premature birth, being of male gender, and prenatal exposure to substances like cigarette smoke or alcohol 
[221]. It is unclear the extent to which these risk factors are associated with defects in neurotransmitter systems within the brainstem (e.g. noradrenaline or 5-HT) [221].

Mounting evidence suggests that infants dying of SIDS have an underlying vulnerability in one or more neurotransmitter systems that puts them at higher risk [221, 237]. Defects within 5-HT neurons that synapse on brainstem and midbrain regions controlling sleep and respiratory, autonomic, and cardiovascular homeostasis occur in the majority of SIDS cases [236, 238, 239]. These defects include increased numbers of immature 5-HT neurons, reduced tryptophan hydroxylase (TPH) and 5-HT content, and reduced expression of inhibitory $5-\mathrm{HT}_{1 \mathrm{~A}}$ receptors $[236,240]$. Our goal is to further reveal how 5-HT system defects contribute to the sudden death of a sleeping infant.

\section{Neurogenic Essential Hypertension}

Hypertension of unknown origin (or essential hypertension (EH)) accounts for more than $90 \%$ of all hypertensive cases. Diagnosis of hypertension is made when diastolic $\mathrm{ABP}$ is $\geq 90 \mathrm{mmHg}$ or systolic $\mathrm{ABP}$ is $\geq 140 \mathrm{mmHg}$ on more than two subsequent medical exams [241]. Understanding the mechanisms of hypertension is critical for the development of targeted therapies to not only lower ABP [242], but also lower its associated cardiovascular events (comorbidities), which include stroke, myocardial infarction, and heart failure [241, 242]. The characteristic reduction in ABP, sympathetic activity, and HR seen during NREM sleep [104] is absent or blunted in some hypertensive patients [243]. There may be a circadian component to $\mathrm{EH}$, in that there is a peak incidence of cardiovascular events during the early morning [244, 245]. Coincidentally, there is also an increased incidence of REM sleep during the early morning period [246]. During REM 
sleep, there is a marked increase in HR and BP instability [104]; in fact, spontaneously hypertensive rats exhibit marked BP surges during REM sleep [247].

Several regions within the brain have been implicated in the circuitry mediating $\mathrm{EH}$, including the monoaminergic circuitry integrated by noradrenaline, adrenaline, dopamine as well as GABA, glutamate, and 5-HT neurons located at the pontine, suprapontine, medullary, and spinal regions. 5-HT has been postulated as one of the mechanisms by which neurogenic hypertension manifests, including both the dorsal and median raphe 5-HT neuronal populations [233]. Indeed, both urapidil (5-HT ${ }_{1 \mathrm{~A}}$ agonist) and ketanserin (5- $\mathrm{HT}_{2 \mathrm{~A}}$ antagonist) are marketed as therapies for $\mathrm{EH}$ [248], although their mechanisms of action are not completely clear. Studies involving these drugs may be confounded by the specificity of urapidil and ketanserin for non-5-HT receptors. For instance, urapidil is also an $\alpha 1$-receptor antagonist. However, the hypotensive effects of urapidil are blocked in the presence of the $5-\mathrm{HT}_{1 \mathrm{~A}}$ antagonist spiroxatrine but maintained in the presence of the $\alpha 1$-receptor antagonist prazosin [163]. Furthermore, pretreatment with ketanserin prevents the increase in sympathetic nerve discharge induced by 2,5Dimethoxy-4-iodoamphetamine (DOI, a 5- $\mathrm{HT}_{2 \mathrm{~A}}$ receptor agonist) [163]. These results suggest, at least in part, that the effects of urapidil and ketanserin are mediated by the 5$\mathrm{HT}_{1 \mathrm{~A}}$ and 5-HT $2 \mathrm{~A}$ receptors, respectively.

\section{9 - Overview of Techniques}

\section{Whole Body Plethymosography}

Use of whole body plethysmography (the barometric method) allows for the assessment of ventilation in freely behaving rodents. Rodents are placed inside a sealed or 
semi-sealed chamber attached to a pressure transducer, which serves to detect changes in pressure within the chamber as the animal breathes. Boyle's law $\left(\mathrm{P}_{1} \times \mathrm{V}_{1}=\mathrm{P}_{2} \times \mathrm{V}_{2}\right)$ is used to calculate the relative volume and pressure changes during breathing -- due to the expansion of air as it is warmed and humidified when moving through the animals' lungs during inspiration. Based on Boyle's law, if temperature is held constant, there is an inverse relationship between the volume and pressure of a gas (e.g. air). The signal from the pressure transducer, i.e. the signal related to the volume and pressure changes that occur during breathing when the air is warmed and humidified during inspiration, is converted from an analog to digital signal, filtered, and amplified. We can use these data to calculate minute ventilation $\left(\mathrm{V}_{\mathrm{E}}\right)$, breathing frequency $(\mathrm{fB})$, and tidal volume $\left(\mathrm{V}_{\mathrm{T}}\right)$ using: 1) the pressure changes within the chamber; 2) chamber water vapor pressure; 3) chamber internal temperature; 4) animal body temperature and 5) the pressure/volume response of a known volume displacement [249, 250].

\section{Sleep/Wake Cycle}

Electroencephalography (EEG) and electromyography (EMG) can be used in the adult mammalian brain to define behavioral states. In our studies in adult rats, we used EEG and EMG electrodes to assess sleep-state. Detailed surgical procedures have been described previously [251]. Briefly, two EMG electrodes are implanted into the dorsal neck muscles. Three EEG electrodes are screwed into the skull, located as follows: the first electrode at $2 \mathrm{~mm}$ rostral to bregma and $2 \mathrm{~mm}$ lateral to the midline, the second electrode at $3 \mathrm{~mm}$ caudal to bregma and $2 \mathrm{~mm}$ lateral to the midline ipsilateral to the first screw, and a "ground" electrode is placed slightly between the two contralateral to midline and caudal 
to bregma. EMG and EEG screws and electrodes are secured in a six-prong pedestal and secured with dental cement. The electrophysiological signals from these EEG and EMG probes are then amplified with a Grass Model 15LT bipolar physiodata amplifier system and recorded in LabChart.

During wakefulness, EEG is dominated by low-voltage fast activity in the beta (16$30 \mathrm{~Hz}$ ) and gamma $(>30 \mathrm{~Hz})$ range. Sleep spindles and slow (delta) waves of large amplitude dominate the EEG activity during non-rapid eye movement (NREM) sleep, spindles around $12-15 \mathrm{~Hz}$. The number and amplitude of slow waves (slow wave activity, $0.5-4.5 \mathrm{~Hz}$ ) is used as a marker of NREM sleep intensity. Rapid eye movement (REM) sleep is characterized by tonically activated EEG, similar to wakefulness, but can be distinguished from wakefulness due to the presence of rapid eye movements and twitching of the limbs, which can be detected using EMG of the neck muscle [5]. In the rodent, EEG activity is discontinuous prior to $\sim \mathrm{P} 12$, and thus EEG activity is generally not used to assess sleep states during this period [252]. However, muscle activity and general behavioral characteristics resembles that of the adult rodent, providing a method for determining sleep state during the neonatal period.

\section{$\underline{1.10}$ - Specific Aims and Hypotheses}

There is a growing body of evidence that suggests a role for central 5-HT in cardiorespiratory homeostasis in rodents. This dissertation sought to investigate the extent to which 5-HT modulates ABP and HR during sleep, the mechanisms associated with the reduced $\mathrm{ABP}$ and $\mathrm{HR}$ phenotype in central 5-HT deficiency in neonatal rats, and the cardiorespiratory plasticity that follows intermittent hypoxia in freely behaving neonatal 
rodents. This project also investigated the extent to which a specific loss of central 5-HT affected $\mathrm{ABP}$ and $\mathrm{HR}$ during sleep and the mechanisms associated with the elevated $\mathrm{ABP}$ found in freely behaving adult rodents lacking central 5-HT. The overarching hypothesis for this dissertation is that central 5-HT is required for the maintenance of $\mathrm{ABP}$ and autonomic tone at rest in both neonatal and adult rodents and that 5-HT neurons are required for the plasticity that follows intermittent hypoxia in neonatal rodents.

\section{Aim 1:}

Assess the role of central 5-HT neurons in the increase in respiration and blood pressure that follows intermittent hypoxia and/or hypercapnia in neonatal rodents.

Hypothesis: A loss of central 5-HT neurons reduces the respiratory plasticity and rise in blood pressure that follows intermittent hypoxia in neonatal rodents.

Rationale: As described above, central 5-HT neurons project widely to brain regions involved in cardiorespiratory control. However, the specific role of 5-HT neurons in the control of ABP at rest and following intermittent hypoxia/hypercapnia (IH/IHH) has not be elucidated in the neonatal period. Respiratory plasticity does occur during the neonatal period [253] and is thought be a protective mechanism against apnea. However, whether this is a 5-HT dependent mechanism in neonatal rodents as seen in adults is not known. We are particularly interested in the role of 5-HT neurons in the acute and longterm regulation of $\mathrm{ABP}$ during and following hypoxia because SIDS is associated with a modest $(\sim 30 \%)$ reduction in 5-HT content and increased numbers of immature 5-HT neurons $[254,255]$. Therefore, we sought to address whether 5-HT neurons would contribute to the plasticity that follows $\mathrm{IH} / \mathrm{IHH}$ during the neonatal period. Ultimately, we 
found that pups lacking 5-HT neurons had a reduced BP and HR compared to saline treated controls. Further, while both groups exhibited vLTF, only pups with a modest loss of 5HT neurons exhibited a rise in MAP, predominately through an elevated sBP. Our data ultimately suggest that 5-HT neurons contribute to resting BP and $\mathrm{HR}$ and the rise in $\mathrm{BP}$ following AIHH but not the ventilatory responses to AIHH.

\section{Aim 2:}

Assess the role of central 5-HT in the control of MAP and HR in neonatal rodents during sleep. Additionally, reveal mechanistically how central 5-HT contributes to autonomic control of MAP and HR in neonatal rodents during sleep.

Hypothesis: A loss of central 5-HT reduces MAP and HR and influences autonomic control of MAP and HR during the neonatal period specifically during QS, when the 5-HT neurons are still firing.

Rationale: As described above, 5-HT neurons help maintain cardio-respiratory homeostasis by projecting to, and modifying the activity of, cardiovascular, respiratory and autonomic neurons in the brainstem and spinal cord [238, 239]. Furthermore, the activity of brainstem 5-HT neurons changes across states of vigilance, being highest in wakefulness and lowest in REM sleep (i.e. active sleep (AS) in infants) [223]. 5-HT neurons also express many other neurotransmitters, including substance P, TRH, enkephalin, and GABA [150, 151]. Some studies have demonstrated that the autonomic dysfunction that occurs in infants that eventually succumb to SIDS may be dependent on states of vigilance $[256,257]$. Therefore, we sought to address the specific role of central 5-HT, including the physiological mechanisms involved, in the control of ABP during sleep in neonatal rodents. 
We found that pups lacking central 5-HT had a reduced ABP and HR, specifically in QS. The ABP phenotype was associated with reduced vascular sympathetic tone, as assessed by a reduced response to phentolamine. The HR phenotype was associated with elevated PSNS tone to the heart, as indicated by a greater rise in HR following i.v. injection with atropine methyl nitrate. These results suggest that central 5-HT is important in maintaining $\mathrm{ABP}$ and HR by elevating sympathetic drive to the vasculature and reducing PSNS to the heart, respectively.

\section{Aim 3:}

Assess the role of central 5-HT in the control of MAP and HR in adult rodents during sleep. Additionally, assess the role of central 5-HT, and the physiological mechanisms involved in the autonomic control of MAP and HR in adult rodents during sleep.

Hypothesis: A loss of central 5-HT leads to dysfunction in the control of ABP and HR in wakefulness and NREM sleep, when 5-HT neurons are active.

Rationale: Similar to Aim 2, which addressed the role of central 5-HT in the control of $\mathrm{ABP}$ during sleep in neonatal rodents, we sought to address whether the effects of a loss of central 5-HT would persist into adulthood. Contrary to our findings in neonatal rodents lacking central 5-HT, we found that in adults, central 5-HT prevents ABP from rising, an effect that is particularly evident in REM sleep. Therefore, we also sought to address the mechanism behind the increased ABP in the face of 5-HT deficiency. Ultimately, we found that neither sympathetic drive nor angiotensin II contribute to the elevated ABP in 5-HT deficiency. 
CHAPTER 2: Plasticity in breathing and arterial blood pressure following acute intermittent hypercapnic hypoxia in infant rat pups with a partial loss of 5-HT $\underline{\text { neurons }}$

Jennifer Magnusson and Kevin J. Cummings

Department of Biomedical Sciences, College of Veterinary Medicine, University of Missouri, Columbia, MO, USA

Running Head: 5-HT neurons and LTF in infant rats 


\section{1 - ABSTRACT}

The role of serotonin (5-HT) neurons in cardiovascular responses to acute intermittent hypoxia $(\mathrm{AIH})$ has not been studied in the neonatal period. We hypothesized that a partial loss of 5-HT neurons would reduce arterial blood pressure (ABP) at rest, increase the fall in ABP during hypoxia, and reduce the long-term facilitation of breathing (vLTF) and ABP following AIH. We exposed 2 week-old, 5,7-dihydroxytryptamine (5,7DHT)-treated and controls to $\mathrm{AIH}\left(10 \% \mathrm{O}_{2} ; \mathrm{n}=13\right.$ control, 14 treated), acute intermittent hypercapnia (AIHC; 5\% $\mathrm{CO}_{2} ; \mathrm{n}=12$ and 11 ), or acute intermittent hypercapnic hypoxia (AIHH; $10 \% \mathrm{O}_{2}, 5 \% \mathrm{CO}_{2} ; \mathrm{n}=15$ and 17). We gave five 5-min challenges of $\mathrm{AIH}$ and $\mathrm{AIHC}$, and twenty $20 \mathrm{sec}$ challenges of AIHH to mimic sleep apnea. Systolic (sBP), diastolic $(\mathrm{dBP})$, mean arterial pressure (MAP), heart rate $(\mathrm{HR}), \dot{V}_{E}$ and metabolic rate $\left(\dot{V}_{\mathrm{O}_{2}}\right)$ were continuously monitored. 5,7-DHT induced an $\sim 35 \%$ loss of 5-HT neurons from the medullary raphe. Compared to controls, pups deficient in 5-HT neurons had reduced resting $\mathrm{sBP}(\sim 6 \mathrm{mmHg}), \mathrm{MAP}(\sim 5 \mathrm{mmHg}), \mathrm{HR}$ (56 beats/min), and experienced a reduced drop in ABP during hypoxia. AIHH induced vLTF in both groups, reflected in increased $\dot{V}_{E}$ and $\dot{V}_{E / V} \dot{\mathrm{O}}_{2}$, and decreased arterial $\mathrm{PCO}_{2}$. The sBP of pups deficient in 5-HT neurons, but not controls, was increased 1-hr following AIHH. Our data suggest that a relatively small loss of 5-HT neurons compromises resting $\mathrm{ABP}$ and $\mathrm{HR}$, but has no influence on ventilatory plasticity induced by AIHH. AIHH may be useful for reversing cardiorespiratory defects related to partial 5-HT system dysfunction. 


\section{2 - INTRODUCTION}

Neonatal mammals are prone to respiratory and cardiovascular instabilities due to relatively immature control systems and hence can experience repeated episodes of hypoxia and hypercapnia. In addition to the ventilatory response, chemo- and baroreflexmediated cardiovascular responses are necessary during hypoxia to increase blood flow to critical tissues in order to meet metabolic demands. The maintenance of arterial blood pressure (ABP) in the face of hypoxia-induced vasodilation is a key component of the integrated response; ultimately any change in ABP depends on the ventilatory, autonomic, and neurohumoral responses to hypoxia [7]. ABP of adult rats typically falls in response to acute hypoxia, notwithstanding the concurrent, baroreflex-mediated tachycardia [258260]. Acute hypercapnia, on the other hand, generally has modest effects on ABP [260, 261]. There have been few studies that have examined the effects of hypoxia and hypercapnia on $\mathrm{ABP}$ in the neonatal period; in lambs, hypoxia elicits a mild decrease in ABP with a concomitant increase in HR [262, 263].

Serotonergic neurons residing within the medullary raphe nuclei help maintain cardio-respiratory homeostasis by projecting to, and modifying the activity of, cardiovascular, respiratory and autonomic neurons in the brainstem and spinal cord [238, 239]. Adult mice with a specific loss of central 5-HT have reduced heart rate (HR) and ABP [224]. In neonatal rat pups, a systemic loss of 5-HT (i.e. from the CNS, autonomic ganglia and blood) leads to a premature, severe loss of ABP during severe hypoxia [148]. However, the specific role of 5-HT neurons in the control of resting ABP, and the ABP responses to moderate hypoxia and hypercapnia in the neonatal period has not previously been explored. 
Acute intermittent hypoxia (AIH) or asphyxia induces an increase in ventilatory and sympathetic motor activity that can persist for hours after the termination of the stimulus (i.e. ventilatory (vLTF) and sympathetic long-term facilitation (sLTF) [227, 264]). It is generally thought that these forms of plasticity are adaptive, ostensibly reducing the chances of apnea and ensuring appropriate oxygen transport to the most metabolically active tissues. There is ample evidence from adult animals that the expression of vLTF depends on, among other factors, serotonergic projections from the medullary raphe to the phrenic motor nucleus $[265,266]$. There is some evidence that SLTF also depends on 5HT [266]. Although vLTF has been shown in neonatal rats in response to AIH [253], the degree to which this phenomenon depends on 5-HT neurons is unknown. Further, the effects of $\mathrm{AIH}$ on the long-term regulation of blood pressure at an age roughly equivalent to infancy (i.e. effects owing at least partially to SLTF) have not been investigated.

We are particularly interested in the role of 5-HT neurons in the acute and longterm regulation of ABP during and following hypoxia because the Sudden Infant Death Syndrome (SIDS) is associated with, among other immunohistochemical defects, a 30\% reduction in 5-HT content and increased numbers of immature 5-HT neurons [254, 255]. In addition, there are records that suggest SIDS cases succumb following one of possibly several hypoxic and hypercapnic episodes [267, 268]. It may be that even a relatively small lesion to medullary 5-HT system predisposes an infant to sudden death because of compromised cardiovascular responses to the hypercapnic hypoxia occurring during sleep apnea, or from a failure to elicit long-lasting cardiovascular and respiratory adaptations to these challenges (i.e. LTF). This study addresses the hypotheses that a partial loss of 5-HT 
neurons exacerbates the drop in $\mathrm{ABP}$ during hypoxia and reduces the magnitude of both vLTF and the increase in ABP following repetitive hypoxic episodes.

\section{$\underline{2.3 \text { - METHODS AND MATERIALS }}$}

\section{Ethical Approval}

All experimental protocols were approved by the Institutional Animal Care and Use Committee of the University of Missouri at Columbia, MO.

\section{Animals and treatments}

We used a total of 37 vehicle (saline) control Sprague-Dawley rat pups along with 39 pups treated with 5,7-dihydroxytryptamine (5,7-DHT). Pups were derived from 10 breeding pairs. Males and females were used, as in preliminary experiments, we did not see any effect of gender. Dams were fed ad libitum on standard rat chow, and kept on a $12 \mathrm{hr}$ light-dark cycle. On any given day, we tested at least one treated and one control littermate.

Surgery (5,7-DHT injection)

5,7-DHT was injected into postnatal day (P)10-12 rat pups, as previously described [269]; experiments were performed 4 days later at P14-16. Prior to 5,7-DHT injection, pups were injected with desipramine (50ul of a $10 \mathrm{mg} / \mathrm{ml}$ solution, in saline, i.p) in order to preserve catecholaminergic neurons. While under $1.5-2 \%$ isoflurane pups were injected (i.c.v., via the cisterna magna) with $3 \mu \mathrm{l}$ of $13 \mathrm{mg} / \mathrm{ml}$ 5,7-DHT (40 $\mu$ g total) or vehicle $(0.9 \%$ saline). We previously found that this dose resulted in an incomplete loss of 5-HT neurons 
(personal observation). After each injection, tissues were sutured and pups were allowed to recover in a small chamber over a heating pad and returned to the dam. Treated pups nearly always lost 1-2 grams in the 24 hrs following surgery, before gaining weight over the following 3 days (albeit at a reduced rate compared to controls). Nevertheless, we observed that treated pups always behaved normally in the litter, with no signs of dehydration, lethargy, or any other behavioural abnormalities.

\section{Surgery (femoral catheter)}

We implanted a femoral catheter into pups four days following 5,7-DHT or vehicle injection (P14-16), as previously described [148]. Prior to surgery, the tip of the PE 10 catheter was heated, stretched to the appropriate diameter, and prefilled with heparinized saline solution $(100 \mathrm{ul} / \mathrm{ml})$. Under $2 \%$ isoflurane a $\sim 1 \mathrm{~cm}$ skin incision was made in the left groin and a 20X dissecting microscope was used to dissect out the left femoral artery. The artery was then tied with 5-0 surgical suture just distal to the epigastric branch. An incision was made on the left femoral artery for insertion of a PE10 catheter. The catheter tip was advanced $\sim 0.6$ to $0.8 \mathrm{~cm}$, close to the inguinal ligament. Tissues were sutured using 5-0 surgical silk.

\section{Experimental Setups}

\section{Whole-body plethysmography}

Similar to previous studies in our lab, we used whole body plethysmography to measure cardiorespiratory variables of interest [148]. For these whole-body measurements, we used a jacketed glass chamber (volume $=100 \mathrm{ml}$ ) attached to a programmable water 
bath/pump (Fisher Scientific, Pittsburgh, PA) to hold chamber temperature constant at 30$31{ }^{\circ} \mathrm{C}$, monitored with a thermocouple (Omega Engineering Inc., Stanford, CT) inserted into the chamber. Air was provided by a wall outlet and hypoxia $\left(10 \% \mathrm{O}_{2}\right.$, balance $\left.\mathrm{N}_{2}\right)$ and hypercapnia $\left(5 \% \mathrm{CO}_{2}\right.$, balance air) from pre-mixed cylinders. Air, hypoxia and hypercapnia were passed through a flask of water to control for the effects of day-to-day variability in humidity on the magnitude of the respiratory signal (see below). Hypercapnic hypoxia $\left(10 \% \mathrm{O}_{2}, 5 \% \mathrm{CO}_{2}\right.$, balance $\left.\mathrm{N}_{2}\right)$ was produced by blending $\mathrm{N}_{2}, \mathrm{O}_{2}$ and $\mathrm{CO}_{2}$ from cylinders. The three gases were passed through a flowmeter with three independent channels and a common output (Cole-Parmer, Vernon Hills, IL). With $\mathrm{O}_{2}$ (AEI Technologies, Pittsburgh, PA) and $\mathrm{CO}_{2}$ analysers (CWE, Inc., Ardmore, PA) we empirically determined the ratios of $\mathrm{N}_{2}, \mathrm{O}_{2}$ and $\mathrm{CO}_{2}$, as well as the duration of their application, that were sufficient to take the $\%$ inspired $\mathrm{O}_{2}$ from $\sim 21$ to $10 \%$, and the $\%$ inspired $\mathrm{CO}_{2}$ from 0 to $5 \%$, in $\sim 20-25 \mathrm{sec}$. This third protocol was designed to more closely mimic blood gas perturbations associated with sleep apnea. All gases were sent through a final flowmeter (Cole-Parmer) in order to regulate the flow to $\sim 300 \mathrm{ml} / \mathrm{min}$ (hypoxia and hypercapnia) or $600 \mathrm{ml} / \mathrm{min}$ (hypercapnic hypoxia). In addition to its higher flow, hypercapnic hypoxia was delivered directly into the chamber without humidification in order to further minimize gas wash-in time. Chamber pressure was kept close to atmospheric by pulling gas from the opposite end of the chamber via wall vacuum, also run through a flowmeter to precisely balance the gas flows. Inflow and outflow gas were run through $20 \mathrm{G}$ needles in order to create a nearly-sealed chamber to maximize the pressure signal related to breathing (i.e. minimizing the "leak" of pressure from the chamber), while still being able to deliver continual flow of gas. In order to determine $\dot{V}_{\mathrm{O}_{2}}$ 
during normoxia, hypoxia or hypercapnia, a separate pump (AEI Technologies, Naperville, IL) pulled a sub-sample of gas through the $\mathrm{O}_{2}$ analyzer, which was single-point calibrated at the start of each experiment with humidified room air to prevent an overestimation of $\dot{V}_{\mathrm{O}_{2}}$ resulting from $\mathrm{O}_{2}$ being diluted with $\mathrm{H}_{2} \mathrm{O}$ from the lung. We could not determine $\dot{V}_{\mathrm{O}_{2}}$ in pups exposed to AIHH because of the high flow rate and short wash-in time for the gas. A differential pressure transducer (Validyne Engineering, Northridge, CA), connected to the animal chamber and a reference chamber was used to ensure the nearatmospheric pressure within the chamber, and to detect changes in chamber pressure related to breathing. The pressure signal generated during inspiration is due to the heating and humidification of the inspired air. To calculate $\mathrm{V}_{\mathrm{T}}$, we used the equation describing the relationship between this pressure signal and the heating and humidification of a volume of air entering the lungs [270]. Body temperatures were measured in a subset of treated and control pups following experimentation, and both were found to be $\sim 36^{\circ} \mathrm{C}$. To account for thermal drift within the chamber during the experiment, the two chambers were connected with a $\sim 10 \mathrm{~cm}$ length of small-diameter tubing. The femoral catheter was fed through a 20G needle and attached to a blood pressure transducer mounted outside the chamber, calibrated at the beginning of each experiment day with a sphygmanometer. Analog signals from respiratory and arterial pressure transducers were fed into a Powerlab data acquisition system (ADInstruments, Colorado Springs, $\mathrm{CO}$ ) and analyzed in LabChart 7.3.7 (ADInstruments).

Experimental Protocol 
We monitored MAP, HR and breathing in treated and control pups at P14-16 (four days following 5,7-DHT injection), at rest and during hypoxia, hypercapnia and hypercapnic hypoxia. Prior to the first challenge, pups were allowed to warm up in the chamber for at least $20 \mathrm{~min}$. We started recordings data when pups displayed full arousal from anaesthesia and full recovery and stability of $\mathrm{HR}$, breathing, $\mathrm{ABP}$ and metabolic rate

$\left(\dot{V}_{\mathrm{O}_{2}}\right)$. We first recorded variables for $15 \mathrm{~min}$ in room air. In a cohort of control and treated pups ( $\mathrm{n}=14$ control and 11 treated from Group 1 and 2, see below) baseline $\mathrm{PaO}_{2}$ and $\mathrm{PaCO}_{2}$ were measured using $~ 90 \mathrm{ul}$ of whole blood, collected via the arterial catheter and immediately transferred to a blood gas analyser (ABL80Flex Co-Ox; Radiometer, Brønshøj, Denmark). Pups were then subjected to one of three protocols: intermittent hypoxia (AIH), hypercapnia (AIHC) or hypercapnic hypoxia (AIHH). Each protocol lasted a total of $120 \mathrm{~min}$. In initial experiments, pups were exposed to five 5-min episodes of AIH (Group 1: $\mathrm{n}=13$ control, 14 treated), or AIHC (Group 2: $\mathrm{n}=12$ control, 11 treated). Episodes were interspersed with $5 \mathrm{~min}$ of room air. Arterial blood gases were measured during the last episode of hypoxia ( $\mathrm{n}=6$ control and 6 treated) or hypercapnia ( $\mathrm{n}=8$ control, 5 treated). A third protocol used a more severe stimulus that better reflects conditions experienced during sleep apnea: Twenty $~ 20-25 \mathrm{sec}$ challenges of intermittent hypercapnic hypoxia (AIHH; Group 3: n=15 control, 17 treated). Each episode of hypercapnic hypoxia was interspersed with 2 min of room air. We were unable to measure blood gases in this group to the transient nature of the stimulus. For all pups we continued recording variables for 60 min following the last challenge. At this point a final arterial blood sample was taken from four pups (2 from each group) to obtain $\mathrm{PaCO}_{2}$ in order to validate observed changes in $\dot{V}_{E}$ 
$\dot{V}_{\mathrm{O}_{2}}$. At the end of the experiment, animals were anesthetized and perfused with $4 \%$ PFA to fix brainstem tissue for immunohistochemistry.

\section{Immunohistochemistry}

Brainstems were cut on a vibratome (VT 1000S, Leica) at 30-40um and freefloating sections were stored in cryoprotectant until immunostaining. The medulla was sectioned starting caudal to the emergence of the raphe obscurus and ending at roughly the dorsal cochlear nucleus. Immunohistochemistry was performed on every sixth section; upon staining, one row of tissue sections was transferred to PBS for washing. Sections were rinsed 3 times in PBS for 5 minutes each. The sections were then blocked with 10\% normal donkey serum (NDS) (Millipore, Temecula, CA) in $0.3 \%$ Triton in PBS for 30 minutes and washed 3 times (5 minutes each) in PBS. Sections were incubated overnight in goat-anti-5-HT (1:1000) (ImmunoStar, Hudson, WI) and mouse-anti-TH (1:1000) (Millipore) in $1 \%$ NDS and $0.3 \%$ Triton in PBS. After the overnight incubation period, sections were washed 3 times in PBS (10 minutes each) and incubated for 2 hours with respective secondary antibodies (donkey anti-goat Alexa 647 and donkey anti-mouse Alexa 488; both 1:200) (Jackson ImmunoResearch, West Grove, PA). After secondary antibody binding, the sections were washed 3 times (10 minutes each) in PBS, mounted on 0.5\% gelatin slides, dried and coverslipped with Prolong Gold (Life Technologies, Grand Island, NY).

Immunohistochemistry images were acquired on an Olympus BX51 microscope containing a three-axis motorized stage (Ludl Electronic Products Ltd., Hawthorne, NY) with a monochrome digital camera (ORCA-AG, Hamamatsu, Bridgewater, NJ) along with 
Neurolucida software (Version 10, MicroBrightField, Willston, VT) using Cy2 and Cy5 filters. Images were acquired individually and were subsequently combined and analyzed with Image J (version 1.46r; National Institutes of Health) for 5-HT and TH containing neurons.

\section{Data and Statistical Analysis}

Baseline variables were determined across 1 min of quiet room air breathing, prior to the first challenge. For Groups 1 and 2 , we calculated respiratory frequency $\left(f_{\mathrm{B}}, \min ^{-1}\right)$, tidal volume $\left(\mathrm{V}_{\mathrm{T}}, \mathrm{ml} / \mathrm{kg}\right), \dot{V}_{\mathrm{E}}\left(f_{\mathrm{B}} \mathrm{x} \mathrm{V}_{\mathrm{T}} ; \mathrm{ml} \cdot \mathrm{min}^{-1} \cdot \mathrm{kg}^{-1}\right), \dot{V}_{\mathrm{O}_{2}}$ (determined using the formula: $\dot{V}_{\mathrm{O}_{2}}=\left(\right.$ fractional $\mathrm{O}_{2}$ inflow - fractional $\mathrm{O}_{2}$ outflow $) \times$ flow $\left(\mathrm{mL} \mathrm{min}^{-1}\right) /$ mass $(\mathrm{kg})$, ventilatory equivalent $\left(\dot{V}_{E} \dot{V}_{\mathrm{O}_{2}}\right)$, systolic arterial blood pressure (sBP), diastolic arterial blood pressure (dBP), mean arterial pressure (MAP, mmHg), and HR (beats.min ${ }^{-1}$ ) from the raw respiratory and $\mathrm{ABP}$ traces. Baseline blood gases, $\mathrm{pH}$ and haematocrit were also determined in a subset of Group 1 and 2 animals. Variables were measured from the last min of each of the 5 normoxic, pre-challenge periods, as well as the last min of each of the 5 min hypoxic or hypercapnic challenges. Due to the short wash-in and wash-out periods of hypercapnic hypoxia (Group 3), and the transient nature of the ABP response, within each of the 20 challenges the maximum $\dot{V}_{E}, \mathrm{ABP}$, and HR responses were measured within a $5 \mathrm{sec}$ window (see Fig. 2.7). Every attempt was made to measure variables from signals that were not contaminated by movement artefact.

For some animals, the arterial pressure signal was lost during the protocol. This was associated with movement and/or a change in the pup's body position within the chamber 
and kinking of the catheter. In addition, the catheters of some control and treated pups became dislodged following movement within the chamber and therefore had to be sacrificed prior to completing the protocol. We indicate animal numbers within the Results and Figures, where appropriate.

The average number of 5-HT-positive neurons within the midline raphe pallidus and obscurus was determined for each pup, using 5-10 sections per animal. Care was taken to ensure that, between groups, counts were done on sections taken from the same rostralcaudal position within the medulla. TH-positive neurons were counted along the extent of the ventrolateral medulla and nucleus of the solitary tract. The rostral-caudal position of each section was confirmed using easily identifiable landmarks (e.g. inferior olives, area postrema, $4^{\text {th }}$ ventricle), and referring to Paxinos and Watson's Rat Brain Stereotaxic Atlas [271].

We analysed the effect of 5,7-DHT on baseline parameters using Student's twotailed t-tests. Effects of each gas and 5,7-DHT treatment on the change in $\dot{V}_{E}, \dot{V}_{\mathrm{O}_{2}}, \dot{V}_{E /}$ $\dot{V}_{\mathrm{O}_{2}}$, MAP, HR, and blood gases were assessed with 2-factor repeated-measures ANOVAs. These tests included data for all animals during the last min of each of the five (or 20) gas challenges and the intervening normoxic, normocapnic periods. To discern whether each gas elicited plasticity (i.e. changes in the variable from baseline to 1-hr postchallenges), we used 2-factor repeated-measures ANOVAs on data from the baseline period and from 1-hr following the last challenge (i.e. only from animals surviving the whole protocol). In line with the changes in $\dot{V}_{E /} \dot{V}_{O_{2}}$, hypocapnia appeared in all four pups (2 control and 2 treated) $1-\mathrm{hr}$ following $\mathrm{AIHH}$; thus $\mathrm{PaCO}_{2}$ data were combined for 
statistical analyses. When significant main effects were found, we used Tukey's post hoc analyses for pair-wise comparisons. Differences between groups with respect to 5-HT- and TH-positive cell counts were determined with a Student's t-test.

\section{4 - RESULTS}

\section{Effects of a partial loss of 5-HT neurons on resting variables}

5,7-DHT treatment reduced the number of 5-HT-positive neurons in the raphe obscurus and pallidus by $33 \%$ and $37 \%$, respectively $(\mathrm{P}<0.001$ for both; Fig. $2.1 \mathrm{a}-\mathrm{c})$, with no effect on the number of TH-positive neurons in the VLM or NTS (Fig. 2.1d). On the day of testing, pups with reduced 5-HT neurons weighed $~ 20 \%$ less than controls (Veh: $31.1 \pm 0.8 \mathrm{~g} ; 5,7$-DHT treated: $24.6 \pm 0.7 \mathrm{~g} ; \mathrm{P}<0.001)$. However, as pups behaved normally following 5,7-DHT injection, this finding is unlikely to be due to gross malnutrition or dehydration. There was a tendency for the $\dot{V}_{\mathrm{O}_{2}}$ of treated pups to be slightly lower than controls, but this effect did not reach statistical significance $(\mathrm{P}=0.06$; Table 1$)$. Treatment had no significant influence on resting $f_{\mathrm{B}}$ (control: $121 \pm 5$; treated: $111 \pm 5$ breaths $/ \mathrm{min}$; $\mathrm{P}=0.14), \mathrm{V}_{\mathrm{T}}(\mathrm{ml} / \mathrm{kg}$, control: $11.3 \pm 0.7 ;$ treated: $11.2 \pm 0.9 ; \mathrm{P}=0.94), \dot{V}_{E}($ Table 1$)$ or $\dot{V}_{E}$ $\dot{V}_{\mathrm{O}_{2}}$ (Table 1). The lack of an effect on $\dot{V}_{E /} \dot{V}_{\mathrm{O}_{2}}$ is also reflected in the lack of an effect of treatment on resting $\mathrm{PaCO}_{2}$ (Table 1). However, baseline $\mathrm{PaO}_{2}$ was slightly but significantly elevated in treated pups compared to controls (Table 1). Finally, HR ( $\mathrm{P}<0.001)$, sBP $(\mathrm{P}=0.01)$ and MAP $(\mathrm{P}=0.01)$ were all significantly reduced in treated pups compared to controls (Table 1). 


\section{Effects of a partial loss of 5-HT neurons on cardio-respiratory responses to acute intermittent hypoxia (Group 1: AIH)}

Typical ABP and HR responses of a control and treated pup to a single episode of hypoxia are shown in Fig. 2.2. While hypoxia induced hypotension in both groups, the loss of $\mathrm{ABP}$ experienced by treated pups was on average $\sim 9 \mathrm{mmHg}$ less than controls (treatment $\mathrm{x}$ hypoxia: $\mathrm{P}=0.02$; Fig. 2.3a), solely because of a reduced drop in sBP (treatment $\mathrm{x}$ hypoxia: $\mathrm{P}=0.002$; not shown). In the $1-\mathrm{hr}$ following hypoxia there were no significant changes in $\mathrm{ABP}$ in either treated or control pups $(\mathrm{P}=0.69$; Fig. 2.3a). In both groups, the drop in ABP was accompanied by tachycardia (Fig. 2.3a). Unlike ABP, a loss of 5-HT neurons had no influence on the magnitude of the HR response (Fig. 2.3a). However, the increase in $\mathrm{HR}$ relative to the fall in $\mathrm{ABP}(\Delta \mathrm{HR} / \Delta \mathrm{BP})$ was more than 3-times greater in 5HT-deficient pups compared to controls ( $\mathrm{P}<0.001$; Fig. 2.3b). Over the 1-hr following AIH, the HR of treated pups remained significantly lower compared to controls, with no evidence of plasticity (Fig. 2.3a).

For both groups, hypoxia elicited a significant increase in $\dot{V}_{E}$. However, control pups had a slightly but significantly greater hypoxic ventilatory response compared to pups deficient in 5-HT neurons (treatment $\mathrm{x}$ gas: $\mathrm{P}=0.03$; Fig. $2.4 \mathrm{a}$ ), solely because the increase in $f_{\mathrm{B}}$ was $\sim 40$ breaths/min greater in controls that in treated pups (treatment $\mathrm{x}$ gas: $\mathrm{P}<0.001$ ).

Relative to baseline values, there was no change in the $\dot{V}_{E}$ of either control or treated pups in the hr following intermittent hypoxia (P=0.11; Fig. 2.4a). Hypoxia had no significant influence on $\dot{V}_{\mathrm{O}_{2}}$ either acutely or 1-hr following the challenges (Fig. 2.4b). The increase in $\dot{V}_{E} / \dot{V}_{\mathrm{O}_{2}}$ across the five hypoxic episodes was not significantly influenced by a loss of 
5-HT neurons (Fig. 2.4c), reflected also by an equivalent decrease in $\mathrm{PaCO}_{2}$ between the two groups during hypoxia (Table 2.2). Compared to baseline, $\dot{V}_{E} / \dot{V}_{\mathrm{O}_{2}}$ was unaltered 1-hr following $\mathrm{AIH}$ ( $\mathrm{P}=0.16$; Fig. 2.4c).

\section{Effects of a partial loss of 5-HT neurons on cardio-respiratory responses to acute intermittent hypercapnia (Group 2: AIHC)}

Acute hypercapnia led to a small but statistically significant increase in $A B P$ $(\mathrm{P}<0.001)$, and this effect was not influenced by a loss of 5-HT neurons (Fig. 2.5). In both groups, $\mathrm{ABP}$ was unaltered 1-hr following $\mathrm{AIHC}(\mathrm{P}=0.75$; Fig. 2.5). Accompanying the increase in ABP during acute hypercapnia was a slight but significant decrease in HR in control and treated pups $(\mathrm{P}<0.001$; Fig. 2.5). There was no significant influence of a loss of 5-HT neurons on $\Delta \mathrm{HR} / \Delta \mathrm{BP}$ (not shown). The HR of control pups remained higher than treated pups 1-hr post-AIHC; however there was no significant change in HR in either group over this period (Fig. 2.5).

In response to acute hypercapnia, $\dot{V}_{E}$ increased to the same extent in both groups (gas: $\mathrm{P}<0.001$; Fig. 2.6a). As was the case with AIH, there was no evidence of vLTF 1-hr following AIHC ( $\mathrm{P}=0.87$; Fig. 2.6a). In both groups there was a slight but significant increase in $\dot{V}_{\mathrm{O}_{2}}$ over the course of the 5 challenges (P<0.001; Fig. 2.6b), but after 1-hr, $\dot{V}_{\mathrm{O}_{2}}$ was not statistically different from baseline values (P=0.13; Fig. 2.6b). As with $\dot{V}_{E}$, acute hypercapnia induced a significant hyperventilation, but 1-hr following AIHC $\dot{V}_{E}$ I $\dot{V}_{\mathrm{O}_{2}}$ had not changed from baseline values $(\mathrm{P}=0.37)$. 


\section{Effects of a partial loss of 5-HT neurons on cardio-respiratory responses to acute intermittent hypercapnic hypoxia (Group 3: AIHH)}

Group 3 animals were exposed to twenty $20 \mathrm{sec}$ episodes of AIHH in order to more closely mimic conditions associated with sleep apnea. Importantly, the total duration of this protocol was the same as AIH and AIHC protocols. We measured the peak ventilatory, $\mathrm{ABP}$ and $\mathrm{HR}$ responses (within a $5 \mathrm{sec}$ window) to each episode of hypercapnic hypoxia (Fig. 2.7). Similar to AIH, AIHH led to a significant drop in ABP (gas: $\mathrm{P}<0.001$, and the drop was smaller in pups with a partial loss of 5-HT neurons compared to controls (treatment x gas: $\mathrm{P}=0.005$; Fig. 2.8a). 1-hr following AIHC, the ABP of control pups had not changed relative to pre-challenge ABP. Surprisingly, however, and unlike the AIH or AIHC protocols, AIHH only induced a rise in sBP and MAP in pups deficient in 5-HT neurons (treatment $\mathrm{x}$ time interaction: $\mathrm{P}=0.001$ and 0.01 , respectively; Fig. $2.8 \mathrm{a}, \mathrm{b} \& 2.9$ ). In both groups, acute AIHH increased HR, but 1-hr after the challenges HR was unchanged from baseline (Fig. 2.8a).

On average, acute hypercapnic hypoxia increased $\dot{V}_{E} \sim 3$-fold, and a partial loss of 5-HT neurons did not influence the response (Fig. 2.10a). Unlike AIH and AIHC, AIHH induced vLTF in both control pups and pups deficient in 5-HT neurons $(\mathrm{P}<0.001$; Fig. 2.10 a,b). In both groups an increase in both $f_{\mathrm{B}}$ and $\mathrm{V}_{\mathrm{T}}$ contributed to vLTF ( $\mathrm{P}=0.02$ for both). In addition, in both groups $\mathrm{AIHH}$ induced a long-term depression of $\dot{V}_{\mathrm{O}_{2}}(\mathrm{P}=0.004$; Fig. 2.10c), a unique feature of this paradigm. As a result, $\dot{V}_{E} / \dot{V}_{\mathrm{O}_{2}}$ nearly doubled in both groups from the beginning to the end of the AIHH protocol ( $\mathrm{P}<0.001$; Fig. 2.10d), reflected also in a significant decline in $\mathrm{PaCO}_{2}$ (Fig. 2.10e). Thus, among our three paradigms, AIHH 
was the only one to elicit long-term plasticity in breathing and blood pressure regulation, and these effects were not dependent on the presence of a full complement of 5-HT neurons. On the contrary, AIHH induced an increase in ABP only in pups deficient in 5HT neurons.

\section{$\underline{2.5}$ - DISCUSSION}

In this study, we treated two-week old rat pups with 5,7-DHT to induce a partial loss of 5-HT neurons, with the goal of modelling the partial 5-HT system dysfunction described in SIDS cases. We examined the consequences of this partial lesion for ABP and the control of breathing during and following acute intermittent hypoxia, hypercapnia and hypercapnic hypoxia. Our hypotheses were that a partial loss of 5-HT neurons would compromise $\mathrm{ABP}$ regulation at rest and in response to acute hypoxia, and that 5-HTneurons are not only important for respiratory LTF but also for the increase in ABP following acute intermittent hypoxia. Most of the previous studies exploring the role of 5HT neurons in cardiorespiratory homeostasis have used rodent models harboring extensive lesions to the medullary 5-HT system [213, 269]. Our findings reveal that cardiorespiratory homeostasis is altered in infant rat pups following even a relatively small ( $\sim 35 \%)$ loss of medullary 5-HT neurons. A loss of these neurons led to reduced resting sBP, MAP and $\mathrm{HR}$, as well as a reduced fall in ABP during acute hypoxia. In addition, we found that AIHH induced respiratory and metabolic plasticity at this age, characterized by increased $\dot{V}_{E}$, decreased $\dot{V}_{\mathrm{O}_{2}}$, and decreased $\mathrm{PaCO}_{2}$. A small loss of 5-HT neurons had no impact on this response. 


\section{Implications for a partial loss of 5-HT neurons on cardiorespiratory homeostasis at rest in infant rat pups}

Despite normal feeding and overall behavior, pups deficient in 5-HT neurons were significantly lighter than controls. Although we have no obvious explanation, we note that a loss of 5-HT neurons or content in the neonatal period is consistently associated with reduced growth rate and body mass $[211,213,269]$. Resting ABP values were similar to those reported previously for rat pups at this age [272]. We found that in resting, normoxic conditions, infant rat pups deficient in 5-HT neurons had reduced sBP, MAP and HR compared to their control littermates. Reduced MAP has been observed in adult mice totally devoid of central 5-HT [224], but to our knowledge this is the first report indicating a role for 5-HT neurons in resting $\mathrm{ABP}$ in rodents of an age roughly equivalent to infancy. Since only sBP was reduced following a loss of 5-HT neurons suggests that at this age, central 5-HT signalling contributes to the sympathetic outflow to the cardiac ventricles, as has been found by others in adult animals [273]. HR of treated pups is significantly reduced compared to controls, a finding that has been previously reported in neonatal rodents deficient in 5-HT neurons [158]. Reduced sympathetic drive to the sinoatrial node could also explain this finding; however, given that 5-HT neurons send projections to nuclei containing cardiac vagal neurons [274], and that 5-HT activates a number of receptors within these nuclei [61], it is equally possible that there is increased vagal drive with a loss of 5-HT signalling. A partial loss of 5-HT neurons had no influence on resting $f_{\mathrm{B}}, \mathrm{V}_{\mathrm{T}}, \dot{V}_{E}$, $\dot{V}_{E} / \dot{V}_{\mathrm{O}_{2}}$, or $\mathrm{PaCO}_{2}$. However, we know from several previous studies that a more extensive loss of 5-HT neurons leads to reduced $f_{\mathrm{B}}$ and apnea $[158,213]$. Interestingly we did find that the $\mathrm{PaO}_{2}$ of treated pups was $\sim 8 \mathrm{mmHg}$ greater than controls. Given there was no effect 
of 5-HT neuron loss on resting $\mathrm{PaCO}_{2}$, it may be that 5- $\mathrm{HT}$ neurons influence the autonomic control of pulmonary vascular resistance and hence the proper matching of lung

$\dot{V}_{E}$ and perfusion. $\dot{V}_{E}$-perfusion mismatch minimally affects $\mathrm{PaCO}_{2}$ because the arteriovenous $\mathrm{PCO}_{2}$ gradient is much smaller than the $\mathrm{PO}_{2}$ gradient.

\section{Effects of a loss of 5-HT neurons on the cardiovascular and respiratory responses to acute hypoxia}

We hypothesized that the fall in ABP elicited by AIH or AIHH would be greater in pups deficient in 5-HT neurons. This hypothesis was based on data from a previous study from our lab in which we systemically injected rat pups with a chemical inhibitor of tryptophan hydroxylase (6-fluorotryptophan, 6-FL) to acutely reduce central 5-HT content. In that study, we found that treated pups had a much greater loss of ABP during repeated episodes of severe hypoxia [148]. However, in the current study we found that pups with reduced 5-HT neurons actually had a reduced fall in ABP in response to moderate hypoxia. This discrepancy may be explained by the treatment (major loss of 5-HT content then, partial depletion of 5-HT neurons now) and/or the severity of the hypoxic stimulus (anoxia then, moderate hypoxia now) used between the two studies. Perhaps more importantly, the systemic application of 6-fluorotryptophan presumably decreased 5-HT levels in the blood, autonomic ganglia and vascular endothelium, tissues that not only store and release 5-HT but that also help regulate vasomotor tone and hence ABP [199].

We also demonstrate that in pups deficient in 5-HT neurons the increase in HR relative to this drop in ABP during hypoxia was $\sim 3$-fold greater than that experienced by controls. Given that the hypoxic stimulus was the same as experienced by control pups 
(Table 2), this finding suggests that the baroreflex is enhanced by a partial a loss of medullary 5-HT signalling. Supporting this idea are studies demonstrating that 5-HT, acting on $5-\mathrm{HT}_{3}$ receptors within the nucleus of the solitary tract, inhibits the cardiac component of the baroreflex [50,275]. As the absolute increase in HR in treated pups was not greater than in controls, it may be that 5-HT neurons influence the sympathetic component of the baroreflex regulating vasomotor tone.

We found that pups deficient in medullary 5-HT neurons exhibited a reduced ventilatory response to hypoxia over the course of the five challenges, due to a reduced hypoxic $f_{\mathrm{B}}$ response. However, we note that neither the increase in $\dot{V}_{E} / \dot{V}_{\mathrm{O}_{2}}$ nor the fall in $\mathrm{PaCO}_{2}$ during hypoxia were affected by a loss of 5-HT neurons. Thus, pups deficient in 5HT neurons hyperventilate to the same degree as controls.

\section{Effects of a loss of 5-HT neurons on the plasticity of breathing and blood pressure}

A major goal of this study was to resolve the effects of a relatively minor loss of 5HT neurons on cardiovascular and respiratory plasticity in response to intermittent hypoxia and/or hypercapnia. Our most salient finding in this regard is that in both control and treated pups alike, AIHH elicited vLTF 1-hr following AIHH. AIHH also induced a long-term

depression of $\dot{V}_{\mathrm{O}_{2}}$. In both groups, the increase in $\dot{V}_{\mathrm{E}}$ and decrease in $\dot{V}_{\mathrm{O}_{2}}$ resulted in a near-doubling of $\dot{V}_{E} / \dot{V}_{\mathrm{O}_{2}}$ from baseline to 1 -hr after the challenges. Arterial blood gas analysis of four samples confirmed that $\mathrm{PaCO}_{2}$ was significantly reduced following $\mathrm{AIHH}$. These findings advance those from previous studies that have demonstrated vLTF in the neonatal period $[232,253,276]$, suggesting that the increase in $\dot{V}_{E}$ following AIHH is not 
simply the result of increased metabolic drive. Rather, AIHH induces a leftward relocation along the isometabolic line defining the relationship between $\mathrm{PCO}_{2}$ and alveolar ventilation, reducing plant gain to help prevent apnea following a transient ventilatory overshoot [277].

The magnitude of vLTF was not influenced by a $~ 35 \%$ loss of 5-HT neurons. And, to our surprise, following $\mathrm{AIHH}$ an increase in $\mathrm{ABP}$ was only observed in pups deficient in 5-HT neurons. This suggests that 5-HT neurons, by some unknown mechanism, actually constrain the development of sympathetic LTF following AIHH. We can only speculate on the underlying mechanisms for this finding. Recently it has been shown that 5-HT signalling through $5-\mathrm{HT}_{7}$ receptors, via PKA activation, can constrain the development of phrenic LTF [278], as can adenosinergic signalling through $\mathrm{A}_{2 \mathrm{~A}}$ receptors [279]. It may be that there is more activity through these pathways in control pups. Alternatively, it may be that, unlike pups deficient in 5-HT neurons, there exists a "ceiling effect" in control pups whereby serotonergic pathways contributing to resting $\mathrm{ABP}$ are saturated and not amenable to plasticity.

\section{$\underline{\text { 2.6 - Methodological Considerations }}$}

Although we were not specifically interested in whether vLTF was a unique effect of hypercapnic hypoxia, it is possible we could have observed vLTF following AIH if we had used the same experimental paradigm; i.e. twenty $\sim 20 \mathrm{sec}$ challenges, rather than five 5-min challenges. Indeed, others have demonstrated vLTF in neonatal rat pups using protocols involving brief exposures to many episodes of hypocapnic hypoxia [253]. In addition, given the technical limitations we encountered, we have not included a group 
exposed only to air alone (i.e. time control). However, the lack of cardiorespiratory plasticity following either AIH or AIHC (protocols of the same duration) suggests minimal influence from time alone on our measured variables.

Although we injected 5,7-DHT into the cisterna magna it is nevertheless possible that midbrain 5-HT neurons, those that send projections to the forebrain, were also lesioned. This is an important caveat given that nuclei in the hypothalamus (e.g. the paraventricular nucleus) project to the medulla and have well-described effects on cardiorespiratory control [280]. In addition, this rostral group of neurons innervates regions of the pons and midbrain that are involved in sleep state transitions and arousal [153], so it may be that the cardiovascular phenotypes we describe in pups deficient in neurons is at least in part due to altered sleep regulation.

Finally, it is worth pointing out that 5-HT neurons co-release Substance P and Thyrotropin-Releasing Hormone, among others, the cardiovascular effects we describe in treated pups may not be specifically due to a loss of 5-HT signalling.

\section{$\underline{2.7 \text { - Perspectives and Significance }}$}

We have shown that even a minor loss of 5-HT neurons reduces the resting HR and $\mathrm{ABP}$ of infant rat pups. Another novel finding is that infant rat pups display cardiovascular and respiratory plasticity following AIHH. Hyperventilation develops 1-hr following the

termination of the stimulus, by way of both increased $\dot{V}_{E}$ and reduced $\dot{V}_{\mathrm{O}_{2}}$. This response effectively stabilizes breathing by reducing plant gain and hence the chances of $\mathrm{PaCO}_{2}$ dropping below the apneic threshold during a transient ventilatory overshoot. We also show that 5-HT neurons are not necessary for the development of this plasticity. On the contrary, 
at this age signaling from 5-HT neurons - by some unknown mechanism - constrains the increase in $\mathrm{ABP}$ following $\mathrm{AIHH}$. Based on these findings, an intriguing possibility is that AIHH can be utilized as a strategy to reverse other cardiorespiratory defects resulting from a loss of, or dysfunction within, 5-HT neurons.

Ultimately, our goal is to better understand the role of 5-HT system dysfunction in the pathophysiology of SIDS. Previous recordings from SIDS cases indicate that infants die during one of likely several severely hypoxic episodes; death is preceded by severe bradycardia and presumably low blood pressure. There is evidence that the vast majority of SIDS cases have one or more 5-HT abnormalities within the brainstem 5-HT system, including a $\sim 30 \%$ reduction in 5-HT content and increased numbers of 5-HT neurons, most of which are of a granular, immature phenotype [255]. Our experiments were therefore designed around the notion that the medullary 5-HT system is partially down-regulated in SIDS. There are other immunohistochemical abnormalities, beyond the increased numbers of 5-HT neurons, that others cite as evidence that the 5-HT system is actually upregulated in SIDS [255, 281]. Nevertheless, we have shown that even a relatively minor depletion of 5-HT neurons alters cardiovascular function in a way that might increase the risk of sudden death for an infant who is confronted with other intrinsic or environmental stressors.

\section{$\underline{2.8 \text { - Acknowledgments }}$}

Funding for this work was provided by an American Heart Association Scientist Development Grant (14SDG18560022; PI: KJC) and University of Missouri (MU) College of Veterinary Medicine Faculty Research Grants. The authors thank Dr. Eileen Hasser 
(MU) for assistance with immunohistochemistry, Dr. Craig Emter (MU) for use of a blood gas analyzer, and Ms. Jane Chen (MU) for technical assistance and animal husbandry. 


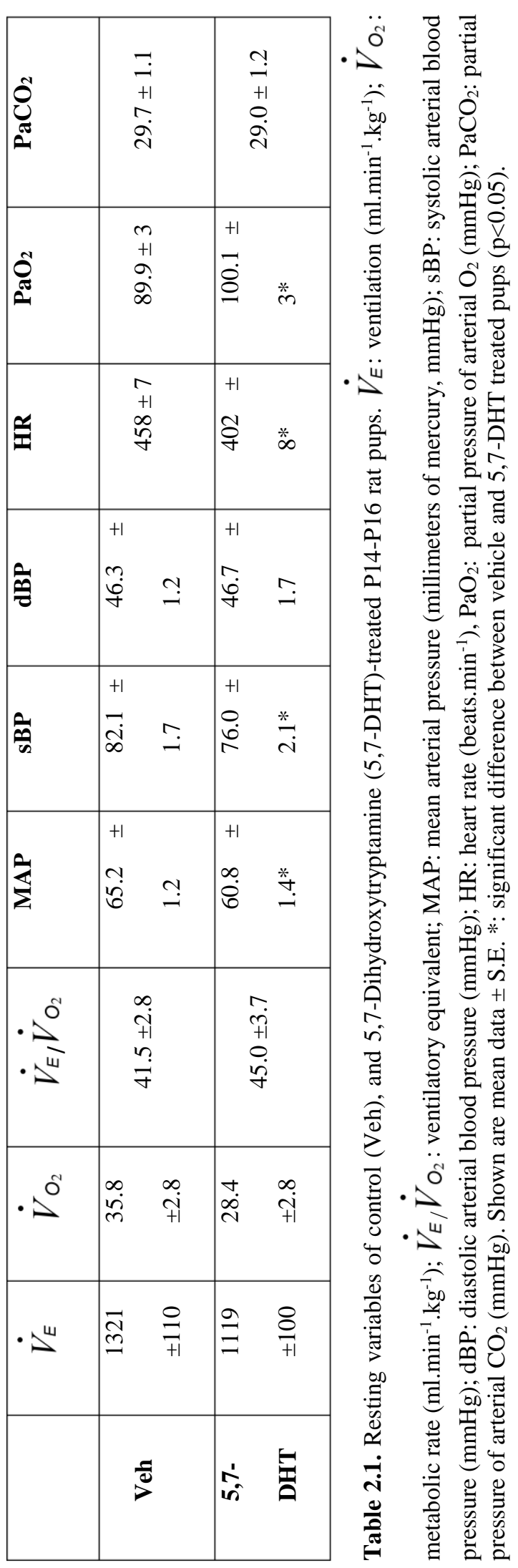




\begin{tabular}{|c|c|c|c|c|c|}
\hline \multirow{5}{*}{$\frac{F}{9}$} & $\nabla$ & 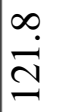 & $\begin{array}{l}0 \\
0 \\
+1\end{array}$ & $\begin{array}{l}+1 \\
\stackrel{+}{N} \\
\text { N }\end{array}$ & $n$ \\
\hline & 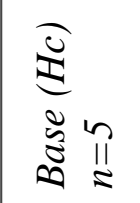 & & & & \\
\hline & $\sum$ & $\begin{array}{l}+1 \\
\stackrel{+}{\sigma} \\
\stackrel{\gamma}{\sigma}\end{array}$ & $\stackrel{\sim}{\check{\gamma}}$ & $\begin{array}{l}+1 \\
0 \\
\\
-\end{array}$ & $\stackrel{\circ}{+}$ \\
\hline & 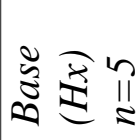 & $\frac{+1}{8}$ & $\begin{array}{l}\infty \\
\sim\end{array}$ & กุ & $\begin{array}{l}0 \\
\dot{+} \\
+1\end{array}$ \\
\hline & 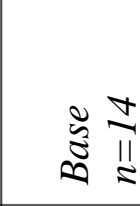 & & & กे & $\frac{\mathfrak{r}}{+1}$ \\
\hline \multirow{5}{*}{$\frac{0}{\frac{0}{0}}$} & $\vec{v}$ & $\frac{n}{n}$ & $\frac{0}{+1}$ & $\begin{array}{l}0 \\
\dot{n} \\
m\end{array}$ & $\begin{array}{l}0 \\
\dot{r} \\
+1\end{array}$ \\
\hline & : & & $m$ & $\stackrel{+1}{\stackrel{n}{n}}$ & 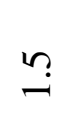 \\
\hline & $\mathbb{Z}$ & $\stackrel{\nabla}{\dot{\sigma}}$ & $\stackrel{n}{+1}$ & $\begin{array}{l}+1 \\
0 \\
0\end{array}$ & $\ddot{m}$ \\
\hline & 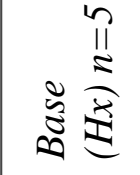 & के & $\begin{array}{l}N \\
\dot{r} \\
+1\end{array}$ & $\stackrel{n}{2}$ & 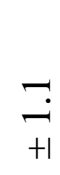 \\
\hline & $\sum_{\infty}^{\infty} \stackrel{\infty}{=}$ & ส่ & $\begin{array}{l}r \\
\\
+1\end{array}$ & ลें & $\underset{+1}{ت}$ \\
\hline & & \multicolumn{2}{|c|}{ ర్ర } & \multicolumn{2}{|c|}{ 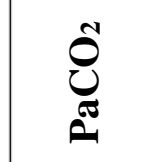 } \\
\hline
\end{tabular}

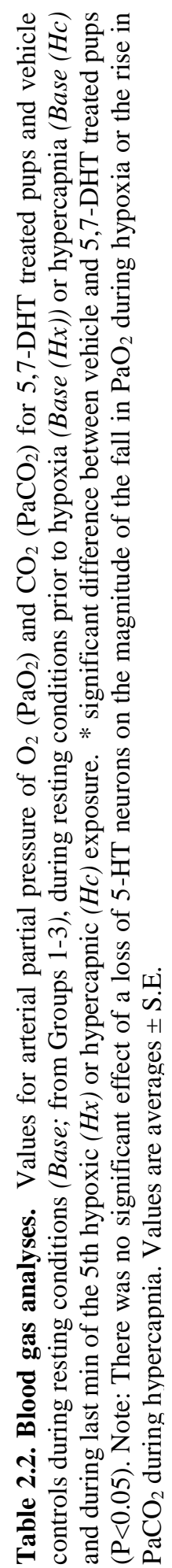


A

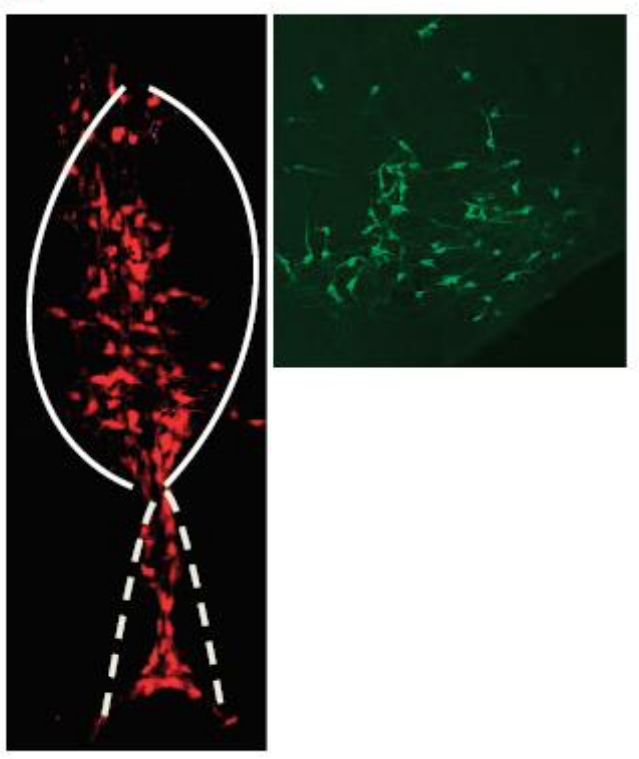

C

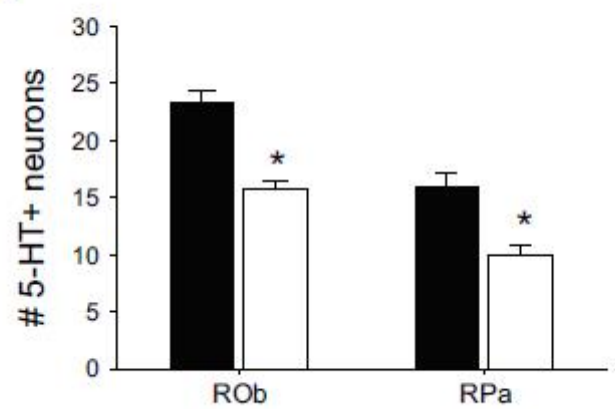

B

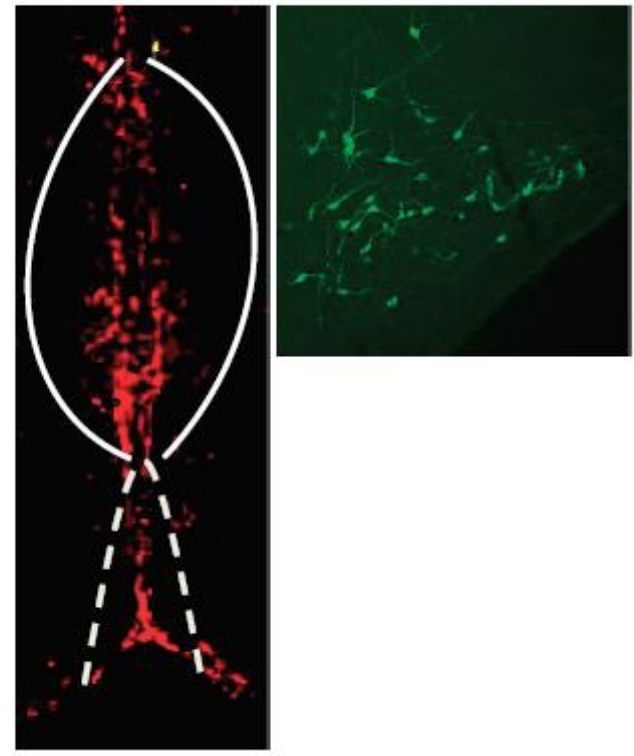

D

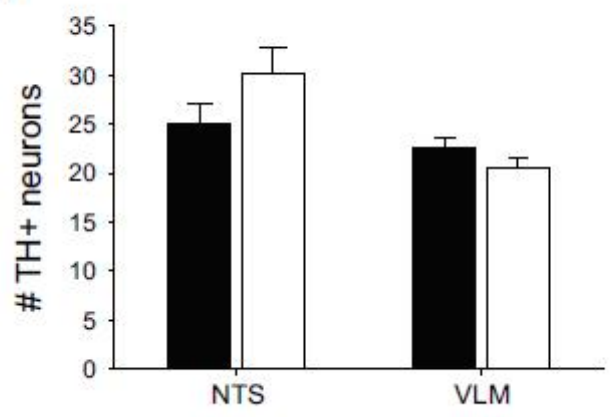

Figure 2.1. Immunohistochemical analyses of medullary slices. (a) Shown are epifluorescent photomicrographs of representative coronal sections (bregma $-12.8 \mathrm{~mm}$ ) stained for 5-HT (red) and tyrosine hydroxylase (green), from control (Veh) $(a)$ and a littermate $(b)$ treated centrally with 5,7dihydroxytryptamine (5,7-DHT). (c) 5,7-DHT treatment resulted in a 33\% and 37\% loss of 5-HT-positive neurons from raphe obscurus (ROb, area within the solid lines), and raphe pallidus ( $\mathrm{RPa}$, area within dashed line), respectively. (d) Compared to control, 5,7-DHT treatment did not significantly affect the number of tyrosine hydroxylase-positive neurons within the nucleus of the solitary tract (NTS) or ventrolateral medulla (VLM). 


\section{Veh}
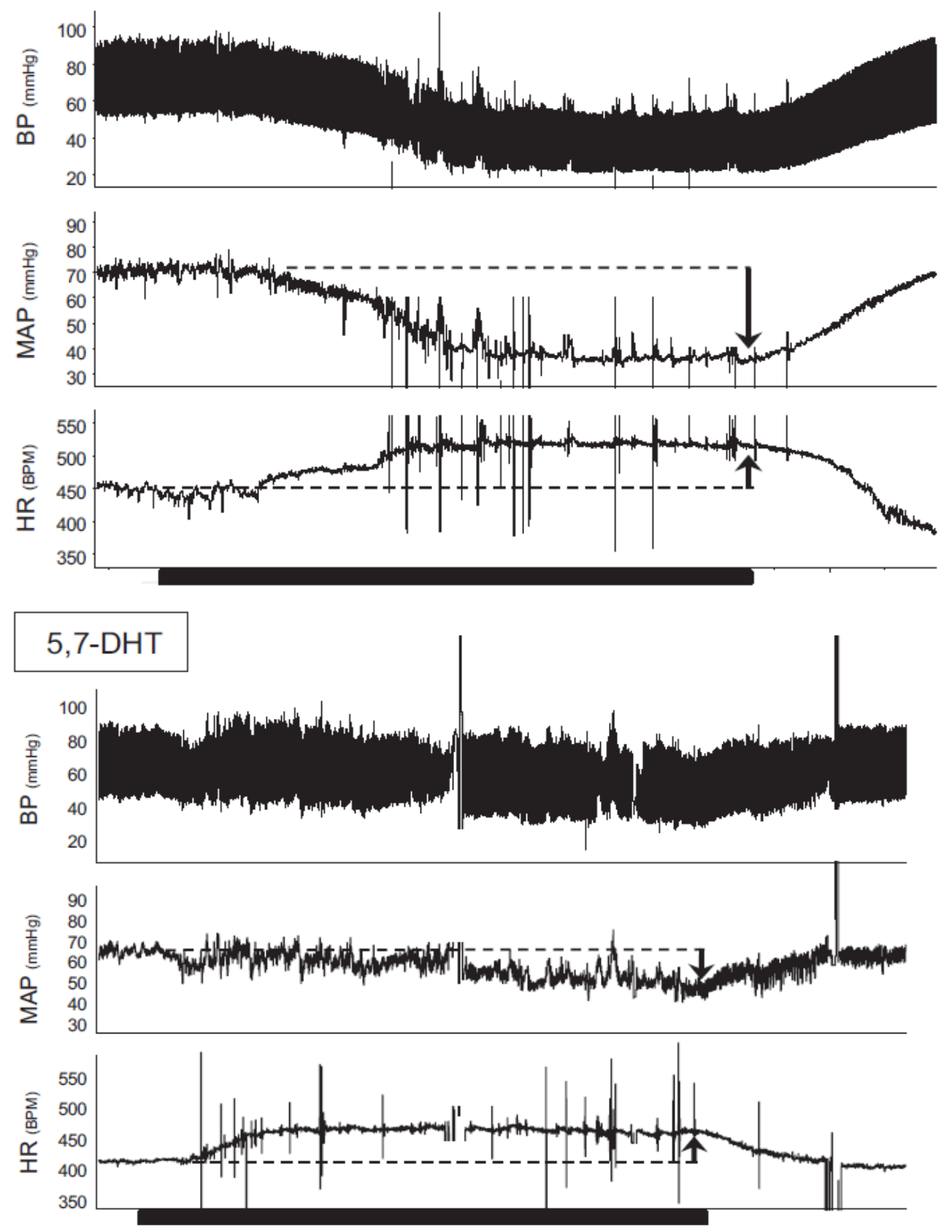

Figure 2.2. Representative traces showing the cardiovascular response to an acute episode of hypoxia. Shown are the responses of arterial blood pressure (ABP), mean arterial pressure (MAP) and heart rate (HR) in a control (top) and 5,7-DHT-treated pup (bottom). 5-min period of hypoxia is denoted with a black bar. Arrows indicate the hypoxia-induced fall in MAP and concomitant increase in HR. 

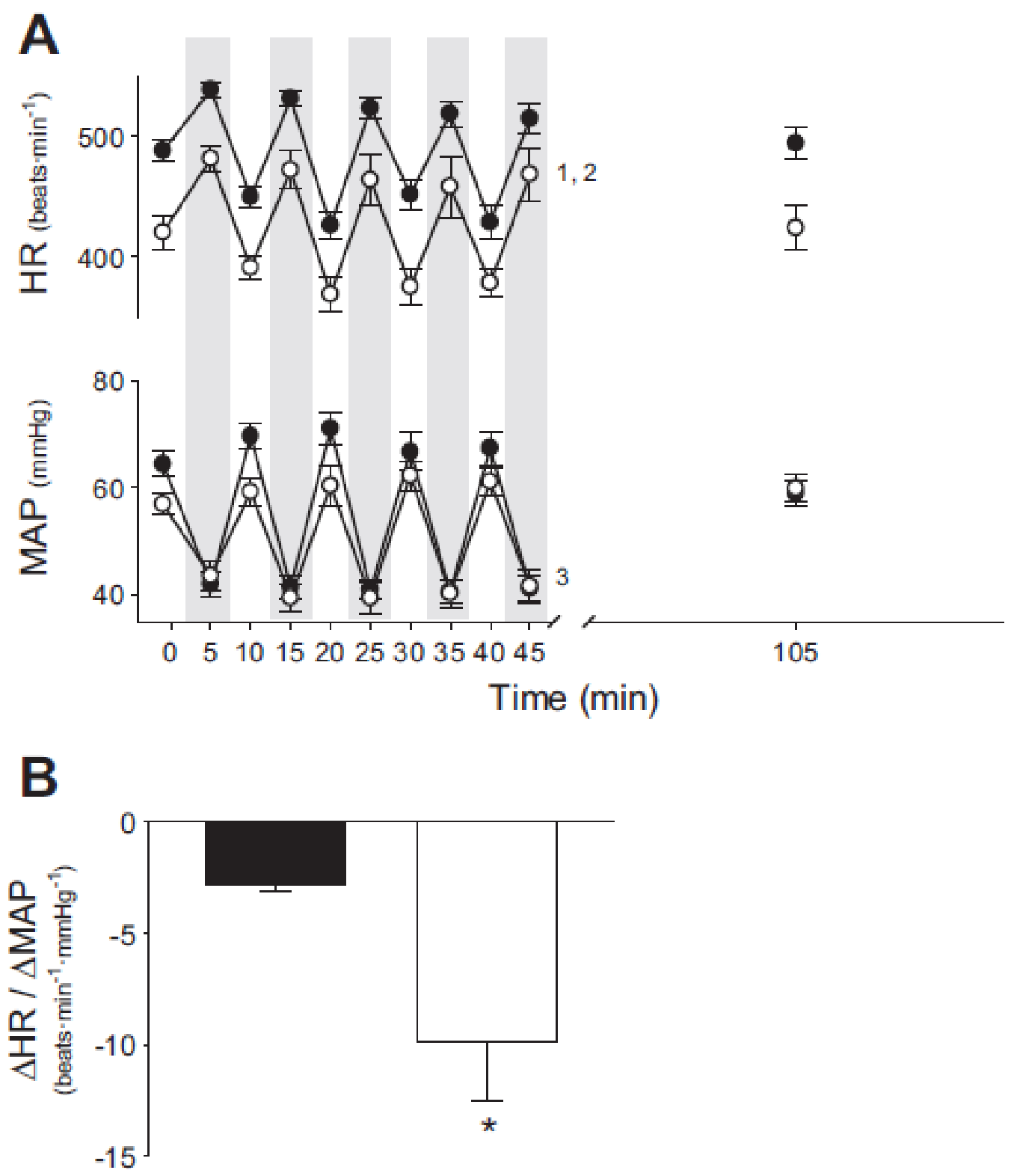

Figure 2.3. Cardiovascular responses to acute intermittent hypoxia (Group 1). (a) Responses of heart rate (HR) and mean arterial pressure (MAP) during each of the five episodes of hypoxia (shaded) the intervening normoxic periods, and 1-hr following the challenges, of vehicle (closed symbols, $\mathrm{n}=13$ ) and 5,7DHT-treated littermates (open symbols, $\mathrm{n}=14$ ). 8 control and 9 treated pups survived the entire protocol and maintained reliable systolic and diastolic BP readings. (b) Change in HR per mmHg change in MAP in response to acute hypoxia. All data are means \pm S.E. ${ }^{1}$ significant effect of treatment; ${ }^{2}$ significant effect of hypoxia; ${ }^{3}$ significant interaction between treatment and hypoxia. Significance evaluated at $\mathrm{P}<0.05$. 

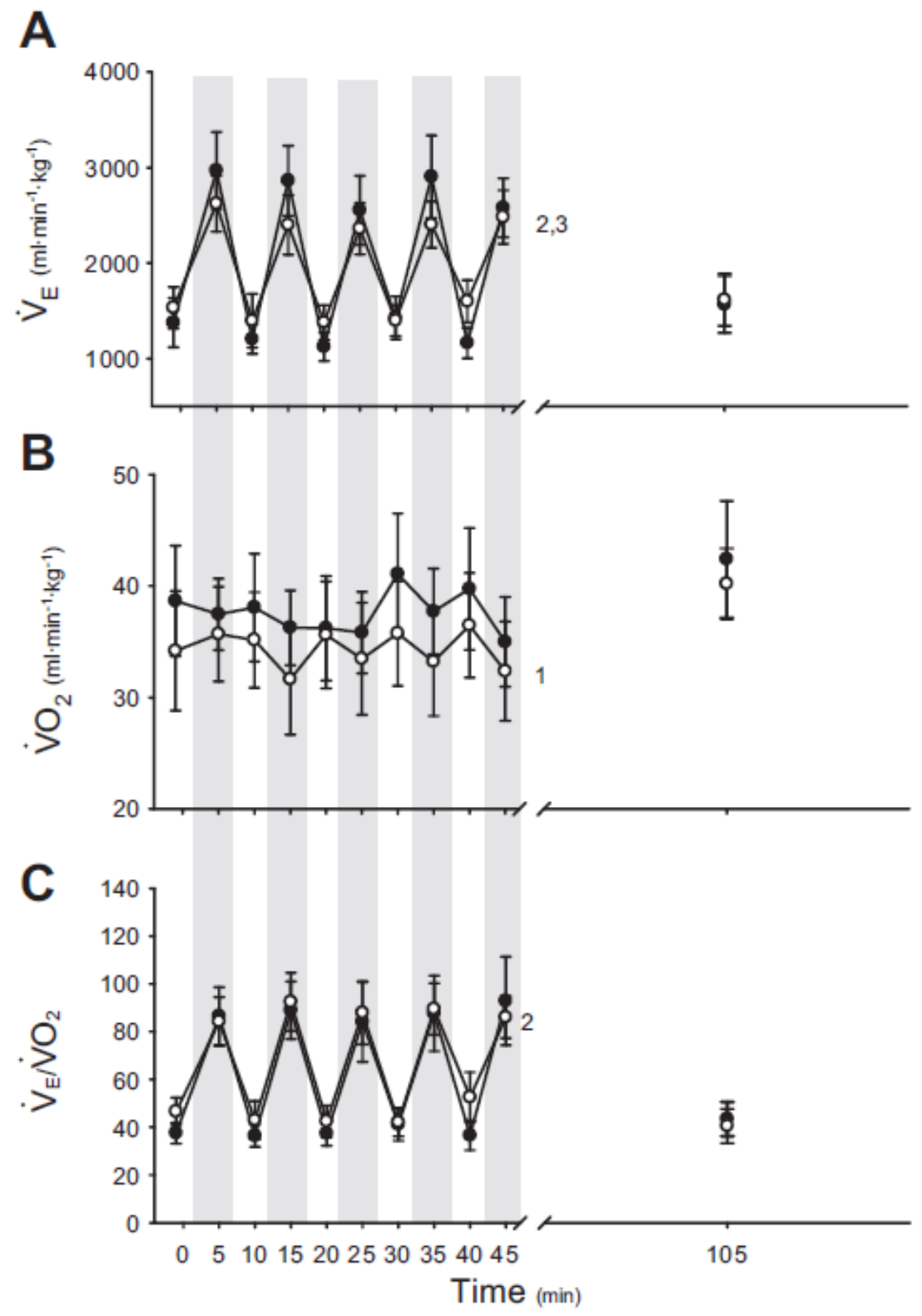

Figure 2.4. Respiratory responses to acute intermittent hypoxia (Group 1). (a) Ventilatory $\left(\dot{V}_{E}\right)$ responses of control (closed circles, $n=13$ ) and 5,7-DHT-treated pups (open circles, $n=14$ ) during each of the five episodes of hypoxia (shaded), the intervening normoxic periods, and 1-hr following the challenges. 11 control and 10 treated pups survived the entire protocol. $b$ ) Responses of metabolic rate $\left(\dot{V}_{\mathrm{O}_{2}}\right)$ in control and treated pups during and following the hypoxic challenges. (c) Ventilatory equivalent $\left(\dot{V}_{E} / \dot{V}_{\mathrm{O}_{2}}\right)$ of control and treated pups during and following the challenges. All data are means \pm S.E. ${ }^{1}$ significant effect of treatment; ${ }^{2}$ significant effect of hypoxia; ${ }^{3}$ significant interaction between treatment and hypoxia. Significance evaluated at $\mathrm{P}<0.05$. 


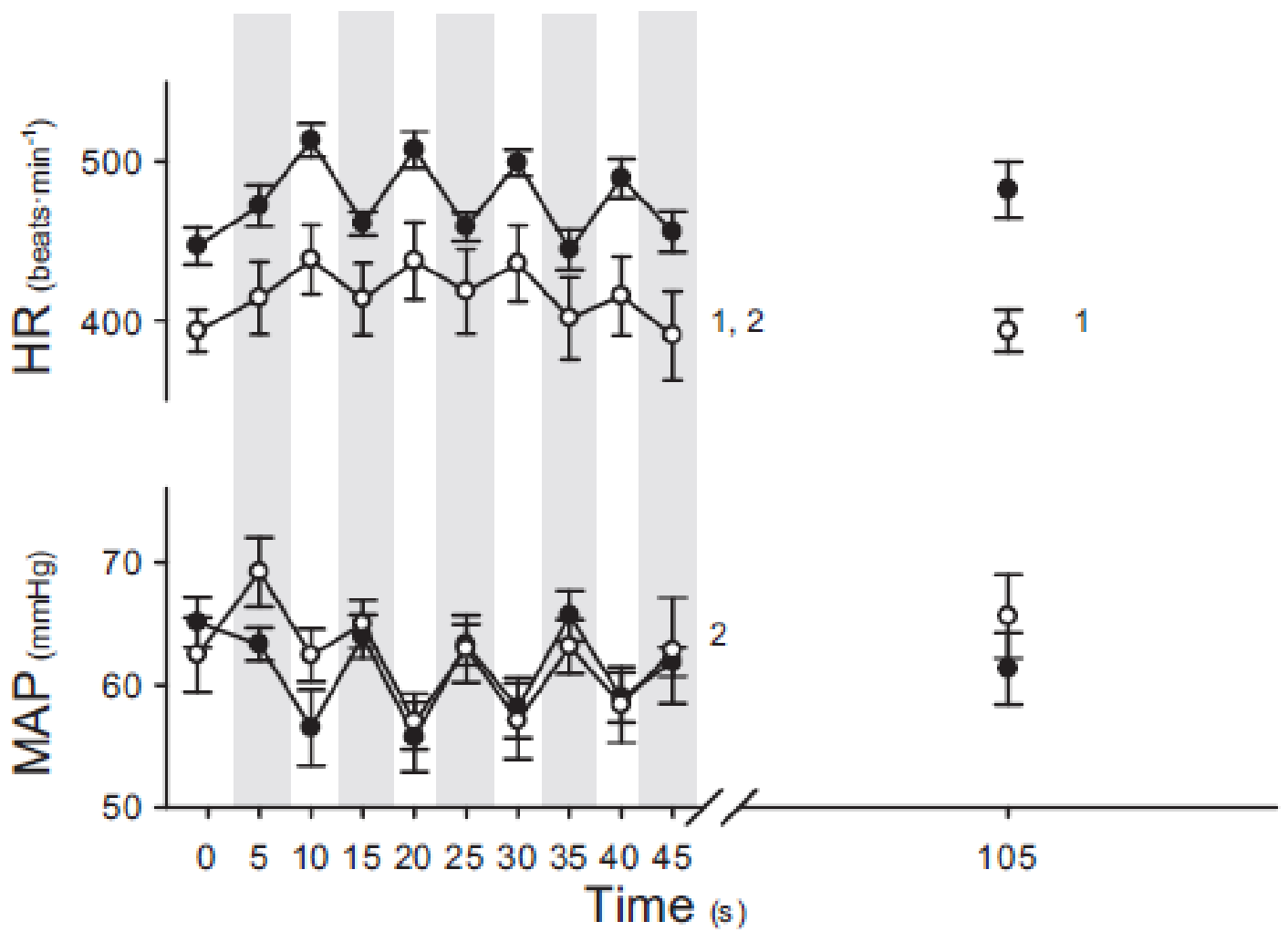

Figure 2.5. Cardiovascular responses to acute intermittent hypercapnia (Group 2). Responses of heart rate (HR) and mean arterial pressure (MAP) during each of the five episodes of hypercapnia (shaded), the intervening normocapnic periods, and 1-hr following the challenges, of vehicle (closed symbols, $\mathrm{n}=12$ ) and 5,7-DHT-treated littermates (open symbols, $n=11$ ). 8 control and 7 treated pups survived the entire protocol and maintained reliable systolic and diastolic BP readings. All data are means \pm S.E. ${ }^{1}$ significant effect of treatment; ${ }^{2}$ significant effect of hypoxia. Significance evaluated at $\mathrm{P}<0.05$. 

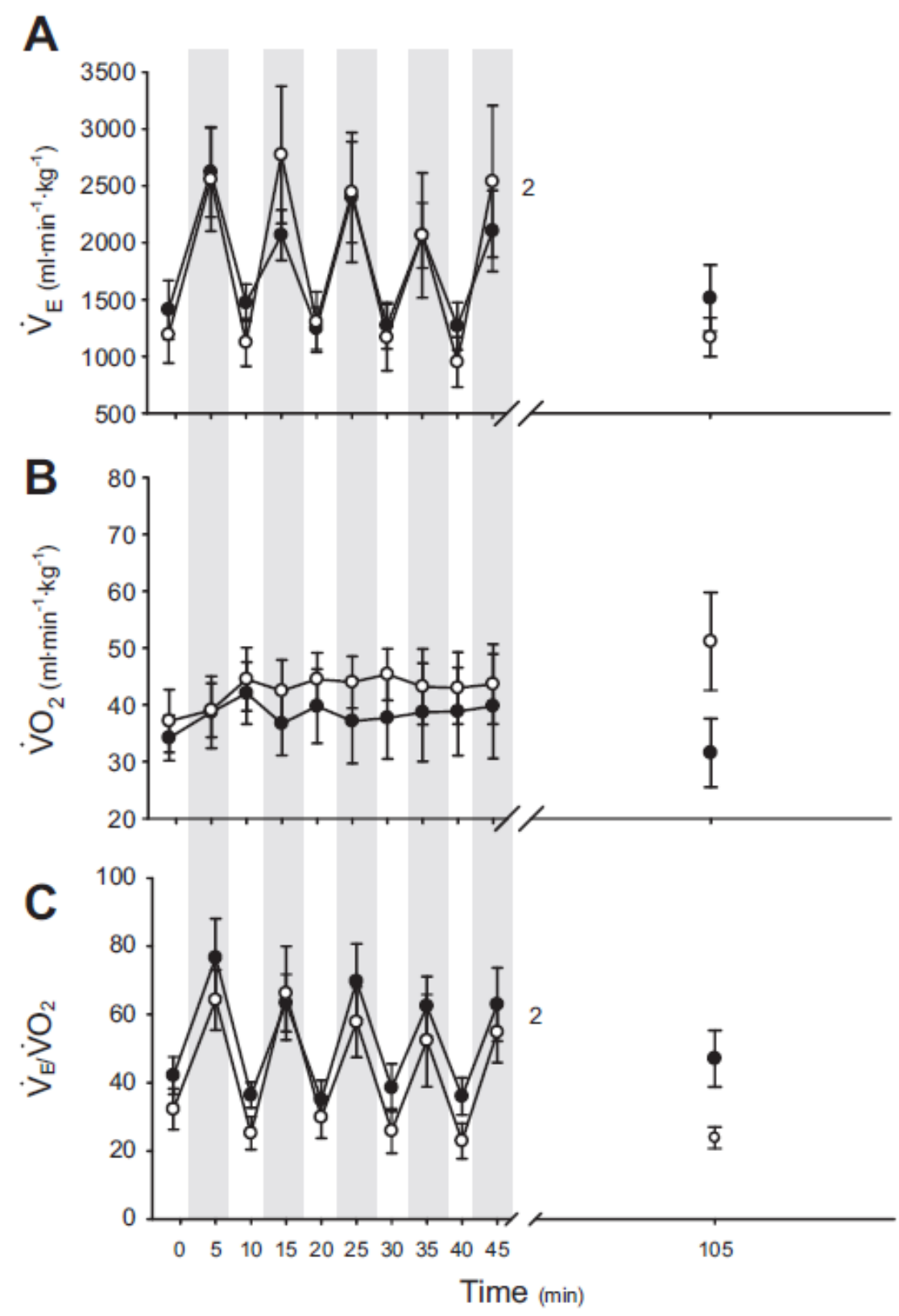

Figure 2.6. Respiratory responses to acute intermittent hypercapnia (Group 2). (a) Ventilatory $\left(\dot{V}_{E}\right)$ responses of control (closed circles, $\mathrm{n}=12$ ) and 5,7-DHT-treated pups (open circles, $\mathrm{n}=11$ ) during each of the five episodes of hypercapnia (shaded), the intervening normocapnic periods, and 1-hr following the challenges. 9 control and 7 treated pups survived the entire protocol. $b$ ) Responses of metabolic rate $\left(\dot{V}_{\mathrm{O}_{2}}\right)$ in control and treated pups during and following the hypercapnic challenges. (c) Ventilatory equivalent $\left(\dot{V}_{E}\right.$ $\dot{V}_{\mathrm{O}_{2}}$ ) of control and treated pups during and following the challenges. All data are means \pm S.E. ${ }^{2}$ significant effect of hypercapnia. Significance evaluated at $\mathrm{P}<0.05$. 

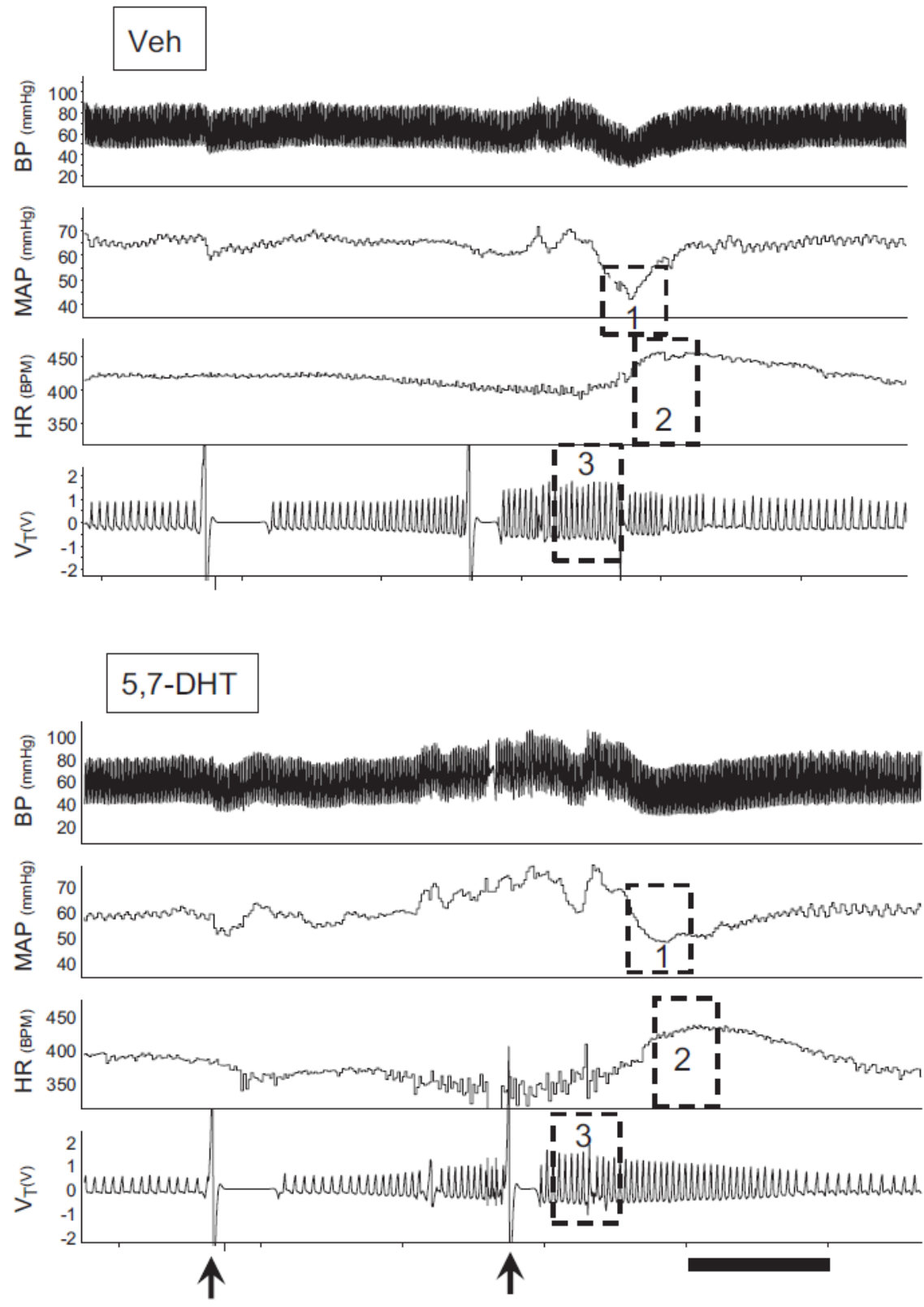

Figure 2.7. Representative traces showing the cardiorespiratory responses to hypercapnic hypoxia. Shown is the raw arterial blood pressure trace (ABP), mean arterial pressure (MAP), heart rate (HR) and breathing $\left(\mathrm{V}_{\mathrm{T}}\right)$ of a vehicle control pup (top) and a 5,7-DHT-treated littermate (bottom) before, during and following an $\sim 20 \mathrm{sec}$ exposure to acute hypercapnic hypoxia. Arrows denote the initiation and termination of the exposure. Note that due to the wash-in time the maximum responses of ABP and HR and breathing are slightly delayed relative to the gas exposure. Boxes indicate the $5 \mathrm{sec}$ segments analyzed for ABP (1), HR (2) and breathing (3). The respiratory trace was not identifiable for $\sim 3 \mathrm{sec}$ when gases were being switched, due to small pressure fluctuations. 

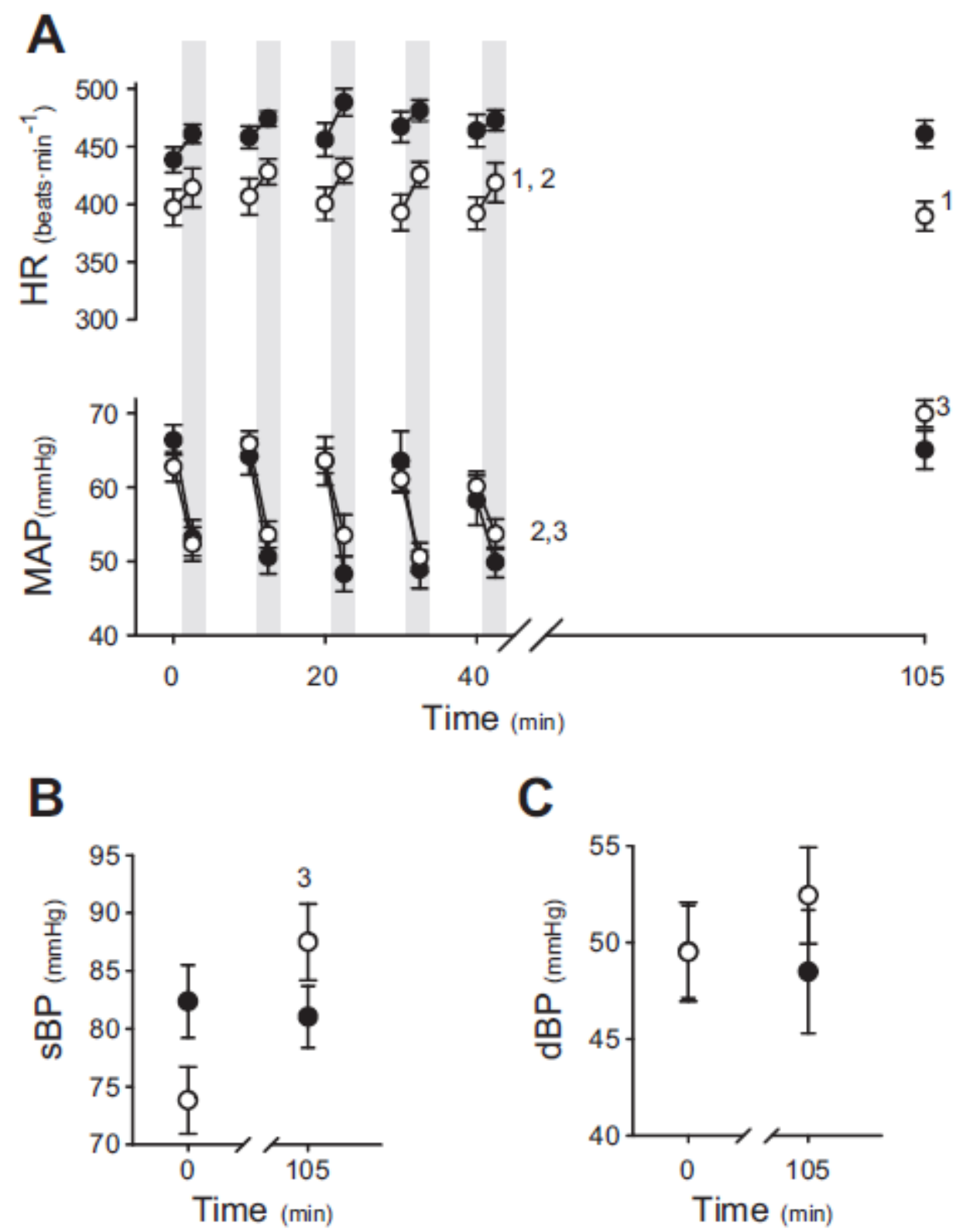

Figure 2.8. Cardiovascular responses to acute intermittent hypercapnic hypoxia (Group 3). (a) Responses of mean arterial pressure (MAP) and heart rate (HR) during the $1^{\text {st }}, 5^{\text {th }}, 10^{\text {th }}, 15^{\text {th }}$ and $20^{\text {th }}$ episodes of hypercapnic hypoxia (shaded), the intervening normocapnic normoxic periods, and 1-hr following the challenges, of vehicle control (closed symbols, $n=15$ ) and 5,7-DHT-treated littermates (open symbols, $n=17$ ). 9 control and 14 treated survived the entire protocol and with reliable systolic and diastolic BP readings. Average systolic (sBP, $b$ ) and diastolic $(\mathrm{dBP}, c)$ arterial blood pressure at baseline (time $=0 \mathrm{~min}$ ) and 60 minutes following the last exposure (time $=105 \mathrm{~min})$. All data are means \pm S.E. ${ }^{1}$ significant effect of treatment; ${ }^{2}$ significant effect of hypercapnic hypoxia. ${ }^{3}$ significant interaction between treatment and hypercapnic hypoxia. Significance evaluated at $\mathrm{P}<0.05$. 


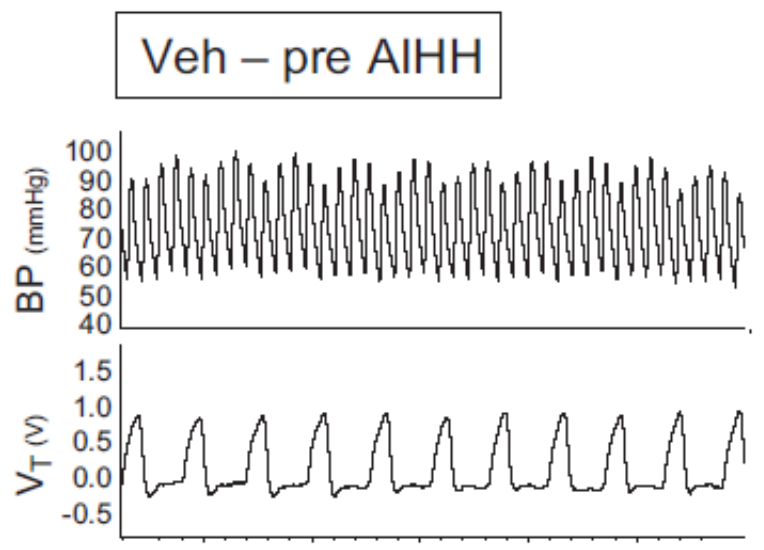

\section{Veh - post AlHH}
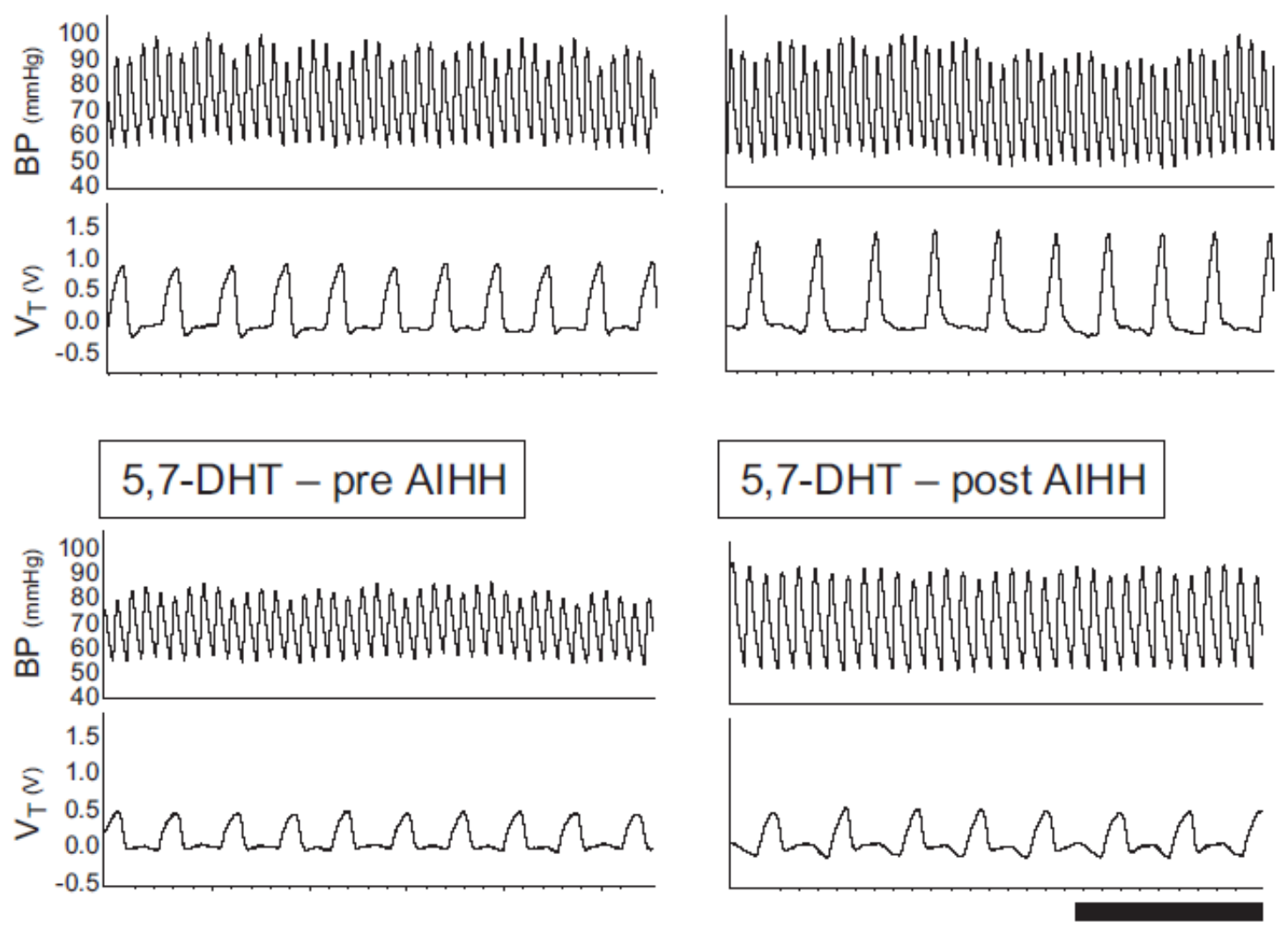

Figure 2.9. Representative blood pressure and respiratory traces pre- and post- acute intermittent hypercapnic hypoxia. Shown are raw arterial blood pressure (ABP) and respiratory volume $\left(\mathrm{V}_{\mathrm{T}}\right)$ traces for a control (top) and 5,7-DHT-treated pup (bottom) prior to (pre-AIHH) and after (post-AIHH) acute intermittent hypercapnic hypoxia. Note the increase in $\mathrm{V}_{\mathrm{T}}$ from pre-AIHH to post-AIHH as well as the increase in systolic blood pressure in the treated pup. 

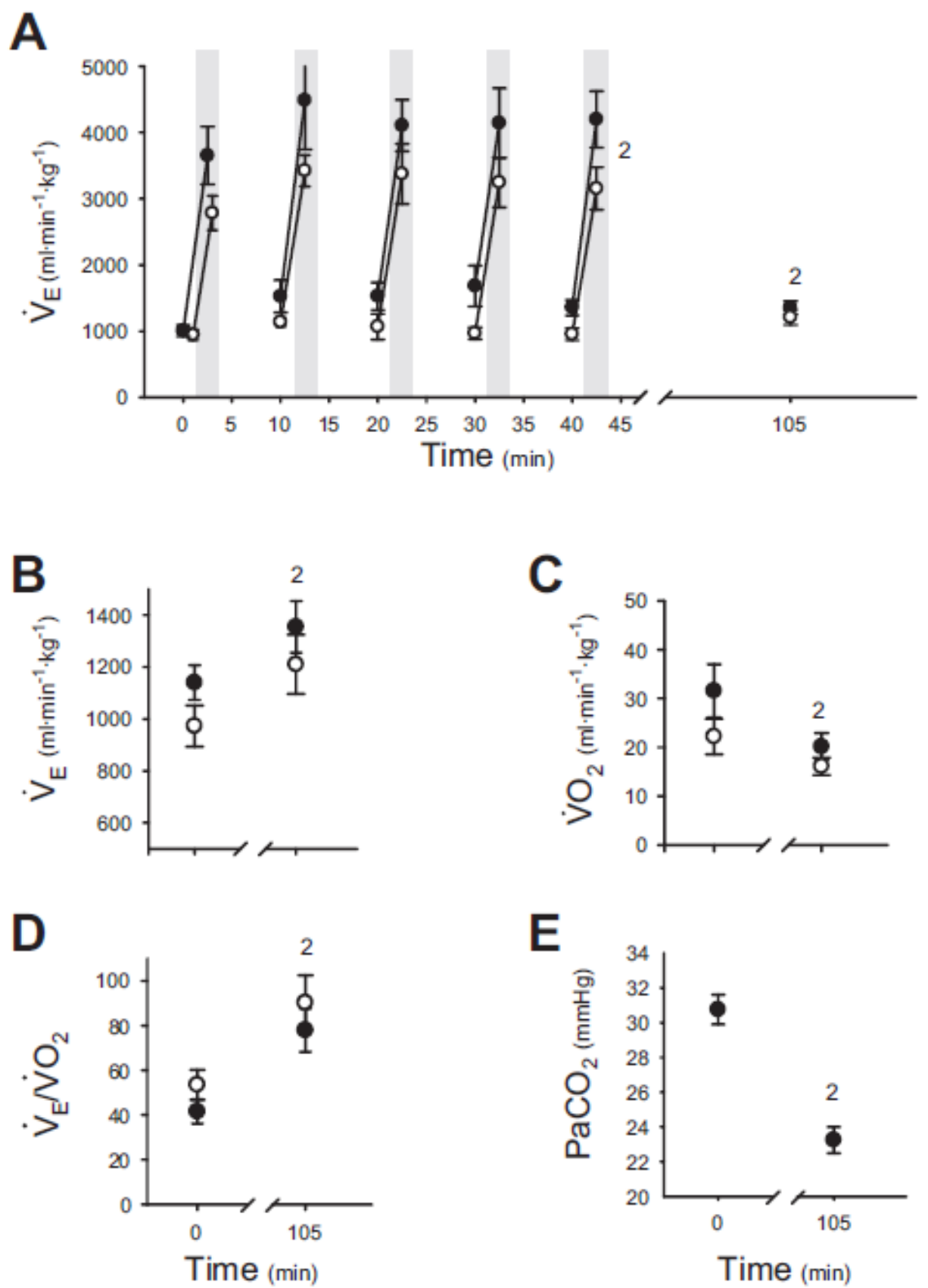

Figure 2.10. Respiratory responses to acute intermittent hypercapnic hypoxia (Group 3). (a) Ventilatory $\left(\dot{V}_{E}\right)$ responses of control (closed circles, $\mathrm{n}=15$ ) and 5,7-DHT-treated pups (open circles, $\mathrm{n}=17$ ) during the $1^{\text {st }}, 5^{\text {th }}, 10^{\text {th }}, 15^{\text {th }}$ and $20^{\text {th }}$ episodes of hypercapnic hypoxia (shaded), the intervening normocapnic normoxic periods, and $1 \mathrm{hr}$ following the challenges. (b) Average $\dot{V}_{E}$ at baseline (time=0 min) and $60 \mathrm{~min}$ after (time $=105 \mathrm{~min}$ ) the last hypercapnic hypoxic exposure. 10 control and 16 treated pups survived the entire protocol. Average metabolic rate $\left(\dot{V}_{O_{2}}, c\right)$, ventilatory equivalent $\left(\dot{V}_{E} / \dot{V}_{O_{2}}, d\right)$ and arterial partial pressure of $\mathrm{CO}_{2}\left(\mathrm{PaCO}_{2}, e\right)$ of control and treated pups at baseline (time $\left.=0 \mathrm{~min}\right)$ and 60 minutes following the last hypercapnic hypoxic exposure (time $=105 \mathrm{~min})$. All data are means \pm S.E. ${ }^{1}$ significant effect of treatment; ${ }^{2}$ significant effect of hypercapnic hypoxia. Significance evaluated at $\mathrm{P}<0.05$. 
CHAPTER 3: Central serotonin and the control of arterial blood pressure and heart $\underline{\text { rate in infant rats: influence of sleep state and sex }}$

Jennifer Magnusson and Kevin J. Cummings

Department of Biomedical Sciences, College of Veterinary Medicine, University of

Missouri, Columbia, MO, USA 


\section{1 - ABSTRACT}

Sudden Infant Death Syndrome (SIDS) is associated with serotonin (5-HT) neuron abnormalities. There is evidence of autonomic dysfunction during sleep in infants eventually succumbing to SIDS, as well as cardiovascular collapse prior to death. Neonatal rodents deficient in central 5-HT display hypotension and bradycardia. We hypothesized that central 5-HT reduces cardiac vagal tone and increases sympathetic vascular tone and, given the firing pattern of 5-HT neurons, that these effects are greater in quiet sleep (QS) than in active sleep (AS). We tested these hypotheses using two week-old, male and female rat pups lacking tryptophan hydroxylase 2 (TPH2-/-) and wildtype littermates (WT). Arterial blood pressure (ABP) and HR were measured over 3-hr, during periods of QS and AS. We also gave atropine or atenolol (each $1 \mathrm{mg} / \mathrm{kg}$ i.v.), or phentolamine $(5,50$ and 500 $\mathrm{ug} / \mathrm{kg}, i . v$.) to separate groups to assess the effects of 5-HT deficiency on autonomic tone to the heart or sympathetic vascular tone, respectively. Compared to WT, male and female TPH2-/- pups had reduced ABP in QS, but not in AS. Atropine induced a greater HR increase in female $\mathrm{TPH} 2-/$ - compared to female WT pups, an effect absent in male TPH2/- pups. Both genotypes experienced the same atenolol-induced drop in HR. In males only, phentolamine induced a smaller decrease in the ABP of $T P H 2-/$ - pups compared to WT. These data suggest that central 5-HT maintains ABP in QS, and HR in both states. In males, central 5-HT facilitates sympathetic vascular tone and in females, reduces cardiac vagal drive. 


\section{2 - INTRODUCTION}

Sudden Infant Death Syndrome (SIDS), the leading cause of death in the postneonatal period, occurs during sleep periods and has a higher incidence in males than females [221]. There is evidence of autonomic nervous system dysfunction in infants eventually succumbing to SIDS - some studies have demonstrated this dysfunction is more apparent in specific states of vigilance $[256,257]$ - and records from SIDS cases indicate that death is preceded by overt cardiovascular collapse (i.e. a substantial loss of heart rate and presumably blood pressure) $[282,283]$. Immunohistochemical and audioradiographic abnormalities within brain stem serotonin (5-hydroxytryptamine, 5-HT) neurons are common in SIDS cases, including an increased proportion of immature 5-HT neurons, reduced tryptophan hydroxylase (TPH) expression and 5-HT content, and reduced 5-HT $\mathrm{HT}_{1 \mathrm{~A}}$ receptors $[236,238,239]$. There is a vast amount of evidence from adult animals that brain stem 5-HT neurons influence the autonomic control of cardiovascular function [284]. In addition, there is mounting evidence that serotonergic defects underpin the autonomic and/or cardiorespiratory dysfunction associated with SIDS. For example, a loss of serotonergic neurons or central 5-HT content in the early postnatal period leads to lower resting arterial blood pressure (ABP) and heart rate (HR), and leads to cardiovascular collapse during exposure to severe hypoxia [148, 156, 158, 211]. This latter phenotype, in particular, is reminiscent of the pathophysiology of SIDS.

The activity of brain stem serotonergic neurons changes across states of vigilance. Their activity is highest in wakefulness and lowest in REM sleep (i.e. active sleep (AS) in infants), with intermediate levels of activity in NREM sleep (i.e. quiet sleep (QS) in infants) [223]. With this in mind, it may be that serotonergic abnormalities underlie not only the 
autonomic and cardiovascular dysfunction apparent in SIDS cases, but also the sleep-state dependency of this dysfunction that has been previously reported [256, 257].

This study addresses two hypotheses; first, that a loss of central 5-HT leads to bradycardia and hypotension due to altered parasympathetic and sympathetic tone to the heart and vasculature, respectively; and second, given the firing pattern of serotonergic neurons, that the influence of 5-HT on ABP and HR are greater in QS compared to AS. To test these hypotheses, we conducted experiments on tryptophan hydroxylase 2 knockout (TPH2-/-) rat pups that have a specific loss of central 5-HT and increased mortality in the second postnatal week (10\%, compared to $1 \%$ for wildtype controls) [155], an age that may be akin to human infancy [285]. Our data lend partial support to these hypotheses and, surprisingly, indicate that the effects of 5-HT on the control of ABP and HR depend on sex. For example, central 5-HT facilitates sympathetic vascular tone in males, but not in females, and reduces cardiac vagal drive in females, but not in males. The implications of these findings for the role of serotonergic dysfunction in male and female SIDS cases is discussed.

\section{3 - METHODS AND MATERIALS}

\section{Ethical Approval}

All experimental procedures were approved by the Institutional Animal Care and Use Committee at the University of Missouri at Columbia, MO, in accordance with national guidelines.

\section{Animals}


This study utilizes tryptophan hydroxylase 2-deficient (TPH2-/-) and wildtype (WT) rat pups at postnatal days 14-16. The generation and genotyping of the TPH2-/- rat line on Dark Agouti background has been previously described [155, 225]. TPH2-/animals have a specific loss of central 5-HT, with unchanged central catecholamines (10). Adult male and female $\mathrm{TPH} 2+/$ - rats were used as breeding pairs to generate WT and TPH2-/- pups. TPH2+/- dams were fed ad libitum on standard rat chow, and kept on a $12 \mathrm{hr}$ light-dark cycle. Pups fed ad libitum until they were removed from the dam for surgery and testing. We used a total of 69 TPH2-/- pups ( $\mathrm{n}=34$ males and 35 females) and 69 agematched WT littermates ( $\mathrm{n}=38$ males and 31 females). TPH2-/- pups weighed significantly less than controls (TPH2-/-: 17.0g $\pm 0.4 \mathrm{~g} ; \mathrm{WT}: 26.7 \mathrm{~g} \pm 0.7 \mathrm{~g} ; \mathrm{p}<0.001)$, an observation that has been previously reported [155, 225].

\section{Determining vigilance state}

Wakefulness, QS and AS were determined using a combination of nuchal electromyography (EMG) along with established behavioral criteria that we and others have previously described $[225,286]$. Specifically, quiet wakefulness $(\mathrm{QW})$ was associated with relatively high EMG amplitude (Fig. 3.1a). When awake, pups typically displayed voluntary, exploratory behavior within the chamber. This unfortunately introduced significant artifacts in the $\mathrm{ABP}$ and $\mathrm{HR}$ records, preventing us from being able to include cardiovascular data from this state. QS was associated with reduced EMG amplitude (Fig. 3.1b), complete immobility without the presence of myoclonic twitching, with the head resting on the forelimbs or floor of the chamber. EMG amplitude decreased further in AS (Fig. 3.1c), when involuntary, myoclonic twitching of the facial muscles, limbs and trunk 
musculature was observed; this muscle activity generated bursts of activity on the nuchal EMG record (Fig. 3.1c).

In preliminary experiments, we noted that the implantation of catheters and EMG electrodes inhibited the appearance of AS, at least in comparison with previously published data from our lab (26). Thus, to facilitate the appearance of AS, we implanted both EMG electrodes and catheters in only a subset of animals ( $\mathrm{n}=6 \mathrm{TPH}-/-; \mathrm{n}=6 \mathrm{WT})$, in order to confirm the behavior normally associated with QS and AS [225]. For all subsequent experiments, we utilized behavioral observation alone (see animal numbers below for Groups 1-4). For EMG electrode implantation, pups were removed from the dam and anesthetized using isoflurane (induction with 3\%, maintained on $2 \%$ ). Noxious paw pinch was used to assess anesthesia depth. Two insulated stainless steel wire electrodes, with suture pads at the end, were used for each animal (Part \#: E363/76, PlasticsOne, Roanoke, VA); pad dimension: Thickness: .66mm; width: 1mm; length: $3.18 \mathrm{~mm}$. Wire dimensions: Diameter: $0.25 \mathrm{~mm}$; length: $37.5 \mathrm{~mm}$. Electrode pads were sutured in place, one under the nuchal muscle and the other in flank muscles (ground). Lidocaine $(\sim 100-200 \mathrm{ul}$ of $20 \mathrm{mg} / \mathrm{ml}$ solution) was applied to each of the electrode sites.

\section{Femoral Artery and Venous Catheterization}

Femoral arterial and venous catheters were implanted into pups immediately prior to testing, as described previously [156]. Briefly, catheters consisting of PE10 tubing were heated, stretched and filled with sterile heparinized $0.9 \%$ saline $(100 \mathrm{ul} / \mathrm{ml})$ prior to surgery. While the pups were under $\sim 2 \%$ isoflurane, a skin incision was made in the left groin for visualization and dissection of the left femoral artery under an X20 dissecting microscope. 
Using 5-0 surgical suture, the artery was tied just distal to the epigastric branch and an incision was made in the artery for insertion of the PE10 catheter. The tip of the catheter was advanced near the inguinal ligament $(\sim 0.6-0.8 \mathrm{~cm})$. Femoral venous catheters were also implanted in pups from Groups 2-4 for drug delivery (see below). Following surgery, lidocaine was applied to the surgical site.

\section{Experimental Setup}

Data were recorded from unrestrained animals while they cycled naturally between wakefulness, QS and AS within a water-perfusable glass chamber (volume: 100ml), attached to a programmable water bath/pump. Chamber temperature, monitored with a thermocouple and digital thermometer (Omega Engineering Inc., Norwalk, CT), was maintained at $31^{\circ} \mathrm{C}$, within the thermoneutral range for pups this age [225, 287]. We have previously demonstrated that under these conditions $\mathrm{TPH}-/$ - and WT pups have the same metabolic rate [225]. Air $\left(21 \% \mathrm{O}_{2}\right.$, balance $\left.\mathrm{N}_{2}\right)$ from a gas cylinder or wall air was passed through a flowmeter prior to entering the chamber via a $20 \mathrm{G}$ needle pushed through one of the rubber stoppers that seal the chamber. Chamber pressure was kept near atmospheric by pulling the gas from the opposite end of the chamber with a pump, also through a 20G needle. Air flow through the chamber was held constant at $300 \mathrm{ml} / \mathrm{min}$. The femoral arterial catheter was exteriorized via a separate $20 \mathrm{G}$ needle and attached to a blood pressure transducer which was calibrated each experimental day with a sphygmomanometer. Analog signals from both arterial pressure and respiratory transducers were fed into a Powerlab data acquisition system (ADInstruments, Colorado Springs, CO) and analyzed in LabChart 7.3.7 (ADInstruments). 


\section{Experimental Groups and Protocols}

Experiments were performed during the daytime $(8 \mathrm{am}-5 \mathrm{pm})$ on four groups of animals. For all groups, animals were allowed to recover from isoflurane anaesthesia for $30 \mathrm{~min}$. In our hands, $30 \mathrm{~min}$ is sufficient for the full recovery of HR, ABP, breathing and metabolic rate $[156,225]$. Pups started displaying QS at this time, and usually the first AS episode occurred within an hour of recovery. Group 1 animals (TPH2-/-: n=11 male, 12 female; WT: $\mathrm{n}=12$ male, 11 female), left unperturbed in the chamber for a total of $3 \mathrm{hrs,}$ were used solely to investigate how central 5-HT deficiency affected ABP and HR in QS and AS, under resting conditions. Animals freely behave in the chamber for 2-3 hrs, in order to acquire data across at least one episode of QS and AS.

Group 2 pups (TPH2-/-: $\mathrm{n}=8$ male, 7 female; WT: $\mathrm{n}=11$ male, 6 female) were used to assess the effects of central 5-HT deficiency on sympathetic tone to the vasculature. Pups naturally behaved in the chamber until an episode of AS was observed (i.e. to ensure pups were cycling naturally between vigilance states). Again, in most animals this occurred within 1-hr. If AS was not observed by $1.5 \mathrm{hrs}$, the experiment commenced regardless. Autonomic tone to the heart was first blocked by simultaneously administering atropine methyl nitrite and atenolol (both at $1 \mathrm{mg} / \mathrm{kg}$, i.v.) (Sigma-Aldrich, St. Louis, MO). Atropine and atenolol were allowed to act for $15 \mathrm{~min}$ in order to reach the steady-state HR response. We then sequentially injected three increasing doses of phentolamine (Sigma-Aldrich), an $\alpha$-adrenoreceptor blocker (5ug/ $\mathrm{kg}, 50 \mathrm{ug} / \mathrm{kg}$ and $500 \mathrm{ug} / \mathrm{kg}$ i.v.), to reduce vascular tone and thus induce a fall in ABP. These doses were determined empirically; at concentrations higher than $500 \mathrm{ug} / \mathrm{kg}$, the drop in blood pressure induced by phentolamine lasted longer than 15 min, making it difficult to give multiple doses to the animal. Animals received 
doses of phentolamine in QS, except for 2 of 17 wildtype and 1 of 15 TPH2-/- pups that had reduced sleep following autonomic blockade and therefore received $5 \mathrm{ug} / \mathrm{kg}$ and 500 $\mathrm{ug} / \mathrm{kg}$ doses while in wakefulness. We gave $15 \mathrm{~min}$ between each dose to allow for recovery of blood pressure. The maximum fall in blood pressure occurred within $\sim 30 \mathrm{sec}$ of each injection. Given the transient nature of the drop in ABP, as well as the fact that all animals woke up upon injection, we were unable to report the ABP response to phentolamine in either QS or AS. In a subset of pups, we injected additional doses of atropine and atenolol $(1 \mathrm{mg} / \mathrm{kg})$ at the end of the experiment; the lack of a change in HR confirmed that autonomic blockade at the level of the heart was maintained throughout the experiment.

Groups 3 (TPH2-/-: n=6 male, 6 female; WT: n=5 male, 7 female) and 4 (TPH2-/$: n=10$ male; 10 female; WT: $n=8$ male, 6 female) were used to assess the effects of central 5-HT deficiency on sympathetic and vagal drive to the heart, respectively. As with Group 2 pups, baseline cardiovascular variables were recorded for $1.5 \mathrm{hrs}$ to allow for the appearance of AS. Subsequently, we administered either atropine methyl nitrate or atenolol, as a 10ul i.v., bolus dissolved in saline (final dose for each: $1 \mathrm{mg} / \mathrm{kg}$ ) during QS. To ensure that drugs acted effectively over the course of the experiment, the same dose of atropine or atenolol was given $\sim 30$ min following the initial injection. HR and ABP were measured over the course of following 1-hr, across multiple episodes of QS and AS (but see below regarding effect of atropine on AS occurrence). Saline alone was injected into a cohort of animals (TPH2-/-: n=6; WT: $n=5)$ to control for the effects of the injection alone on ABP and HR.

Some WT and TPH2-/- did not display any AS episodes following atropine treatment. Despite the reduction in animals numbers, we still report the change in HR in 
AS (TPH2-/-: n=4 males, 3 females; WT: n=5 males, 6 females). At the end of each experiment, intrinsic $\mathrm{HR}\left(\mathrm{HR}_{\mathrm{INT}}\right.$; i.e. HR following complete autonomic blockade) was determined by administering atenolol to pups treated initially with atropine, and atropine to pups treated initially with atenolol.

As we did not observe any significant differences in resting cardiovascular variables between the four groups, the pre-drug periods from animals in Groups 2-4 (1-1.5 hrs) were combined with Group 1 data (3-hr recordings) for analyses (total animals for baseline variables in QS and AS (Fig. 3.2) - TPH2-/-: n=34 male, 35 female; WT: $n=38$ male, 31 female).

\section{Data and Statistical Analysis}

For all experimental groups, we recorded raw pulse pressure, from which systolic (sBP), diastolic (dBP) and HR were derived. We recorded and analyzed cardiovascular variables following the 30 min settling period, from QS and AS episodes lasting at least 10 sec in duration. Statistical analyses on variables of interest were performed using the averages for each animal across each sleep state. Significant effects of genotype, sleep state and sex on sleep variables (\# and duration of AS and QS episodes), resting MAP, sBP, dBP and HR (and interactions) were resolved using 3-factor, repeated measures analyses of variance (3FRMA) (IBM SPSS software, Armonk, NY). Given that state was the withinanimal factor, 3FRMA was performed only on pups displaying both QS and AS (TPH2-/: n=17 male, 19 female; WT: $n=28$ male, 23 female). 2-factor ANOVA (2FA) (Sigmaplot 12.5) was used to assess the effects of genotype and sex on ABP and HR within QS and AS, using the data collected from all animals, irrespective of whether they experienced an 
AS episode or not (TPH2-/-: n=34 male, 35 female; WT: $n=38$ male, 31 female). Effects of genotype and sex on the change in HR following atropine and atenolol treatment were resolved with 2FA (Sigmaplot 12.5). Effects of genotype, sex and the dose of phentolamine on the drop in MAP were resolved with a 3FRMA (IBM SPSS software) and 2FRMA (Sigmaplot 12.5) within each sex. When significant main effects were resolved, pairwise multiple comparisons were performed using Tukey's post hoc analyses. Regression analyses were also performed to examine the potential relationship between cardiovascular variables of $T P H 2-/$ and their reduced body mass.

\section{4 - RESULTS}

Sleep architecture during baseline conditions

We analyzed the number of AS and QS episodes, the duration of these episodes, as well as the \% total time Group $1 \mathrm{WT}$ and TPH2-/- pups spent in AS and QS (Table 3.1). Most animals displayed at least one episode of AS and QS (1/8 male WT, 1/9 female WT; 2/7 male $T P H 2-/$ and 1/10 female TPH2-/- did not experience an AS episode). Both male and female $\mathrm{TPH}$-/- animals had significantly fewer episodes of QS and AS over the 3-hr period (3FRMA, genotype: $\mathrm{p}=0.01$ ), and had longer episodes of AS compared to WT littermates $(\mathrm{p}=0.05)$. However, as they had fewer episodes of QS and AS, TPH2-/- pups spent $\sim 10-15 \%$ more time in wakefulness compared to WT (3FRMA, genotype $\mathrm{x}$ state: $\mathrm{p}<0.001)$.

Central 5-HT maintains arterial blood pressure during quiet sleep 
We hypothesized that central 5-HT prevents ABP and HR from falling during QS, when serotonergic neurons are firing and releasing synaptic 5-HT [223]. There was no significant influence of genotype on sBP, dBP, or MAP during AS (Fig. 3.2a-c; Table 3.2). On the other hand, the sBP, dBP and MAP of $T P H 2-/$ - pups were all significantly reduced during QS, compared to WT ( $<0.03$ for all; Fig. 3.2a-c, Table 3.2). The effect of genotype on blood pressure emerged in QS because, in WT pups, pressure was elevated in QS compared to AS, whereas in $\mathrm{TPH} 2-/$ pups pressure was unaffected by state (genotype $\mathrm{x}$ state interaction: $\mathrm{p}<0.001$ for all; Fig. 3.2a, Table 3.2). Our statistical analyses indicated that both male and female $T P H 2-/$ pups had reduced MAP compared to WT controls (genotype $\mathrm{x}$ sex interaction: $\mathrm{p}=0.06$ ). However, on average, the difference in MAP between male TPH2-/- and WT pups during QS $(\sim 7 \mathrm{mmHg})$, was greater than the difference between female TPH2-/- and WT pups ( 2 mmHg) (Fig. 3.2a; Table 3.2).

Central 5-HT deficient pups had reduced HR compared to WT but, unlike MAP, the magnitude of this effect did not depend on sleep state. In both states, the HR of male and female $T P H 2-/$ - pups was $~ 50-100$ beats/min lower than WT littermates $(\mathrm{p}<0.001$ in both states; Fig. 3.2d). Similar to ABP, the HR of WT pups was higher in QS compared to AS, whereas the HR of $\mathrm{TPH} 2-/$ - was not influenced by state (genotype $\mathrm{x}$ state interaction: p $<0.001$; Fig. 3.2d).

\section{Central 5-HT facilitates sympathetic vascular tone in infant males}

To test the hypothesis that central 5-HT facilitates sympathetic vascular tone in QS, we treated male and female $T P H 2-/$ and WT littermates with increasing doses of phentolamine $(5,50$ and $500 \mathrm{ug} / \mathrm{kg}$, i.v. in saline) to block vascular $\alpha 1$-adrenoreceptors, 
reduce vascular tone and decrease ABP. Surprisingly, the ABP response to phentolamine not only depended on genotype, but also on sex. Representative traces from male and female WT and TPH2-/- pups are shown in Fig. 3.3a and 3.3b, demonstrating the fall in pressure induced by $500 \mathrm{ug} / \mathrm{kg}$ phentolamine. In males, this dose of phentolamine induced a larger decrease in the MAP of WT pups $(\sim 13 \mathrm{mmHg})$ compared to TPH2-/- littermates $(\sim 8 \mathrm{~mm} \mathrm{Hg})(\mathrm{p}<0.001$; Fig. 3.3c). In contrast, female pups experienced the same drop in MAP irrespective of genotype $(\sim 12 \mathrm{mmHg}$; Fig. 3.3b and 3.3c). Across all three doses, male TPH2-/- pups had reduced sensitivity to phentolamine compared to male WT (2FRMA, genotype $x$ dose: $p=0.004$; Fig. 3.3c), whereas the sensitivity of female pups did not depend on genotype (2FRMA, genotype $\mathrm{x}$ dose: $\mathrm{p}=0.70$; 3FRMA, genotype $\mathrm{x}$ dose $\mathrm{x}$ sex interaction: $p=0.04$; Fig. $3.3 \mathrm{c}$ ). The injection of saline alone had no significant effect on MAP (TPH2-/- in QS: $3 \pm 2 \mathrm{mmHg}$; WT in QS: $-1 \pm 1 \mathrm{mmHg}$ ).

\section{Central 5-HT reduces cardiac vagal drive in infant females}

To test the hypothesis that central 5-HT maintains HR in QS, we treated both TPH2/- and WT pups with atropine and atenolol. Like the ABP response to phentolamine, the HR response to atropine depended on genotype and sex. The rise in HR following i.v. administration of atropine was greater in female TPH2-/- pups compared to female WT, an effect not observed in male TPH2-/- animals. In both QS and AS, the HR of female TPH2/- pups increased $\sim 50$ beats/min more than that of WT littermates (genotype $\mathrm{x}$ sex $\mathrm{p}=0.016$ and 0.028 in QS and AS, respectively; Fig. 3.4c), an effect unrelated to their smaller body size $\left(\mathrm{R}^{2}=0.20 ; \mathrm{p}=0.22\right)$. These data suggest that central 5 -HT reduces vagal drive to the heart in females, but not in males. There was no effect of either genotype $(\mathrm{p}=0.38)$ or sex 
$(\mathrm{p}=0.73)$ on the HR response to atenolol (nor was there any significant interaction $(\mathrm{p}=0.24))$. The injection of saline alone had no significant effect on HR (TPH2-/- in QS: $8 \pm 13$ beats/min; WT in QS: $-1 \pm 11$ beats/min). Intrinsic HR (HR INT $)$ was revealed in each group following complete autonomic blockade with atropine and atenolol. HR INT $_{\text {of }}$ male and female TPH2-/- pups was lower than their WT counterparts (genotype: $\mathrm{p}<0.001$; Fig.3.4d). Regression analyses showed a relatively weak, but significant correlation between the body mass of TPH2-/- pups and their HR $\mathrm{INT}_{\text {; }}$ smaller pups had lower HR $\mathrm{INT}_{\mathrm{IN}}$ compared to larger pups $\left(\mathrm{R}^{2}=0.27 ; \mathrm{p}=0.04\right)$.

\section{$\underline{3.5}$ - Discussion}

In this study we investigated whether the bradycardia and hypotension evident in pups deficient in central 5-HT manifests in a sleep state-dependent fashion, and whether sympathetic or parasympathetic dysfunction might underlie these phenotypes. We addressed two specific hypotheses: first, that central 5-HT reduces vagal drive to the heart and facilitates sympathetic vascular tone, and second, that central 5-HT has a greater influence in QS, when serotonergic neurons fire, compared to AS when the neurons are silent. Our experiments lend partial support to these hypotheses. First, both male and female TPH2-/- pups had reduced MAP in QS, but not in AS; HR, on the other hand, was lower in TPH2-/- pups in both sleep states. Second, we showed evidence that cardiac vagal drive was higher and sympathetic vascular tone lower, in $T P H 2-/-$ pups compared to controls. Another important, unexpected finding was that the degree to which central 5-HT influenced autonomic drive to the heart and vasculature depended on sex. Central 5-HT 
facilitates sympathetic vascular tone only in males, and inhibits cardiac vagal drive only in females.

\section{Central 5-HT maintains arterial blood pressure in QS but not AS}

We previously showed that $\mathrm{ABP}$ was reduced in two week-old rat pups following a partial loss of 5-HT neurons [156]. As 5-HT neurons synthesize and release numerous neuromodulators, the current study is the first to demonstrate that 5-HT is necessary for the maintenance of ABP in infancy. Previous studies examining the role of 5-HT in ABP regulation in adult animals have produced varying results. Alenina and colleagues showed that adult male and female $T P H 2-/$ - mice have low MAP and HR [224]. Interestingly, the phenotypes of adult $\mathrm{TPH} 2 /$ - mice are most obvious in the afternoon and early evening and, generally, occur coincidentally with decreased activity. Although here we did not ask about a circadian influence, we show that in males and female pups alike, a loss of central 5-HT leads to reduced MAP solely in QS. The difference in MAP between the genotypes during QS exists essentially because the MAP of WT pups is elevated in QS compared to AS, whereas no such increase occurs in $T P H 2-/$ pups. This observation strongly suggests that the increase in MAP experienced by WT pups in QS is due to increased release of synaptic 5-HT by serotonergic neurons coincident with their increased firing in QS, an event that does not occur in 5-HT-deficient TPH2-/- animals [223].

\section{Central 5-HT facilitates sympathetic drive to the vasculature in males}

Statistically our data suggest that a loss of central 5-HT affects the MAP of males and females to the same degree in QS (sex x genotype: $\mathrm{p}=0.06$ ). However, it may be that 5-HT 
makes a stronger contribution to the regulation of MAP in males compared to females. We showed, for example, that the vascular component of sympathetic drive is facilitated in males by the action of central 5-HT, evidenced by the larger drop in ABP following phentolamine treatment in WT compared to littermate $T P H 2$-/ pups. In fact, phentolamine essentially eliminated the difference in ABP between the two genotypes that existed prior to treatment. This effect was absent in females. This finding is consistent with pharmacological data obtained from adult cats [288]; vascular resistance significantly increased in these animals following the application of a $5-\mathrm{HT}_{2 \mathrm{~A}}$ receptor agonist to the rostral ventrolateral medulla (RVLM), without any chronotropic or inotropic effects on the heart [288]. Reduced vascular resistance in male $T P H 2-/$ - pups may therefore be due to a lack of $5-\mathrm{HT}_{2 \mathrm{~A}}$ receptor signaling in the RVLM. But why is sympathetic vascular tone reduced only in male TPH2-/- pups? At this point we can only speculate. We may assume that the expression of $\alpha 1$-adrenoreceptors in vascular smooth muscle is not influenced by a loss of central 5-HT (although this needs testing). Differences in the levels of sex hormones, even at these young ages, could be a contributing factor. The reduced vascular tone of $\mathrm{TPH} 2 /$ - males may be related to the vasodilating properties of testosterone [289] which, even at these young ages, is found at higher concentrations in males compared to females [290]. Still, an interaction between central 5-HT and estrogens in male pups cannot be fully discounted; indeed, there may be slightly reduced plasma estradiol in males compared to females at these ages, and the sympathoinhibitory role of estrogens have been well described [291]. Exactly how sex-specific factors influence the function of central 5HT in the control of blood pressure awaits further investigation. 


\section{Central 5-HT inhibits cardiac vagal drive in females}

We showed that in both QS and AS, pups deficient in central 5-HT have reduced HR compared to WT. We then assessed the extent to which each arm of the autonomic nervous system contributed to this cardiac phenotype. We demonstrated that in both states, the atropine-induced rise in HR was greater in female TPH2-/- compared to WT pups (an effect not observed in male TPH2-/- pups), suggesting that in infant females, a loss of central 5-HT ultimately leads to enhanced vagal drive to the heart, reducing HR in both states. There are multiple 5-HT receptors expressed on cardiac vagal neurons, including inhibitory $5-\mathrm{HT}_{1 \mathrm{~A}}$ receptors, and it has been shown that $5-\mathrm{HT}_{1 \mathrm{~A}}$ signaling can suppress excitatory glutamatergic drive to these neurons following hypoxic and hypercapnic conditions [168]. Recently we showed that TPH2-/- pups experience prolonged apnea during AS, and hypoventilate in both states [225]. Increased glutamatergic drive to cardiac vagal neurons, subsequent to chronic hypoxia and hypercapnia, may therefore contribute to the increased vagal drive and bradycardia displayed by female, 5-HT-deficient animals. Of course, this does not explain why there is increased vagal drive solely in females. There is relatively recent data suggesting the estrogens are capable of depolarizing cardiac vagal neurons in the nucleus ambiguus, via $G$ protein-bound estrogen receptors [292]. As mentioned, serum estradiol concentrations may be higher in two week-old female rats, compared to age-matched males [290]. While speculative, it may be that a loss of inhibitory 5- $\mathrm{HT}_{1 \mathrm{~A}}$ signaling tips the balance towards estrogen-mediated excitation, increasing vagal nerve activity.

Unlike sympathetic drive to the vasculature, there was little influence of central 5HT on sympathetic drive to the heart, given that the drop in HR following atenolol 
treatment was the same in TPH2-/- and WT pups. Following complete autonomic blockade, HR remained lower in both male and female TPH2-/- pups compared to WT, indicating reduced $\mathrm{HR}_{\mathrm{INT}}$ in $\mathrm{TPH} 2-/-$ pups. That there is a small, yet statistically significant correlation between HRINT and the body size of TPH2-/- pups implies that there are yet-tobe identified developmental factors that also contribute to the lower resting HR of TPH2/- pups.

\section{6 - Perspectives and Significance}

SIDS remains a leading cause of infant death, and occurs more frequently in males than females. Perhaps as high as $\sim 70 \%$ of SIDS cases have at least one defect within the serotonergic system of the brainstem, including reduced 5-HT and expression of TPH2 [221]. There is considerable evidence of autonomic and cardiovascular dysfunction in infants eventually succumbing to SIDS [256, 257, 282, 283, 293]. These infants ultimately succumb during periods of sleep; in some cases, death follows overt cardiovascular collapse [282, 283]. A better understanding of how 5-HT contributes to cardiovascular control during sleep is therefore important in our efforts to reduce SIDS incidence, including the potential for 5-HT to function differently in males than females, or between different states of vigilance.

Herein we show that in males, central 5-HT increases vascular tone to prevent a fall in arterial blood pressure in quiet sleep (Fig. 3.5a). Further, in females, our data suggest that central 5-HT dampens vagal drive in order to increase heart rate (Fig. 3.5b). Given that $\mathrm{TPH} 2-/-$ rat pups are smaller than wildtype controls, it may be that altered development within the CNS or in peripheral tissues contributes to these phenotypes [294]. However, 
we note that at the same age, rat pups in which 5-HT neurons are acutely lesioned with 5,7dihydroxytryptamine (a selective 5-HT neurotoxin) also have reduced ABP and HR. Thus, it seems likely that 5-HT operates physiologically to facilitate the maintenance of ABP and HR in infancy during sleep. Serotonergic dysfunction may therefore put male infants at risk during quiet sleep, especially in the presence of other factors that could lower vasomotor tone and/or arterial blood pressure (e.g. severe hypoxia). Females may be at risk during both quiet and active sleep, due to inappropriately elevated vagal drive and bradycardia. 


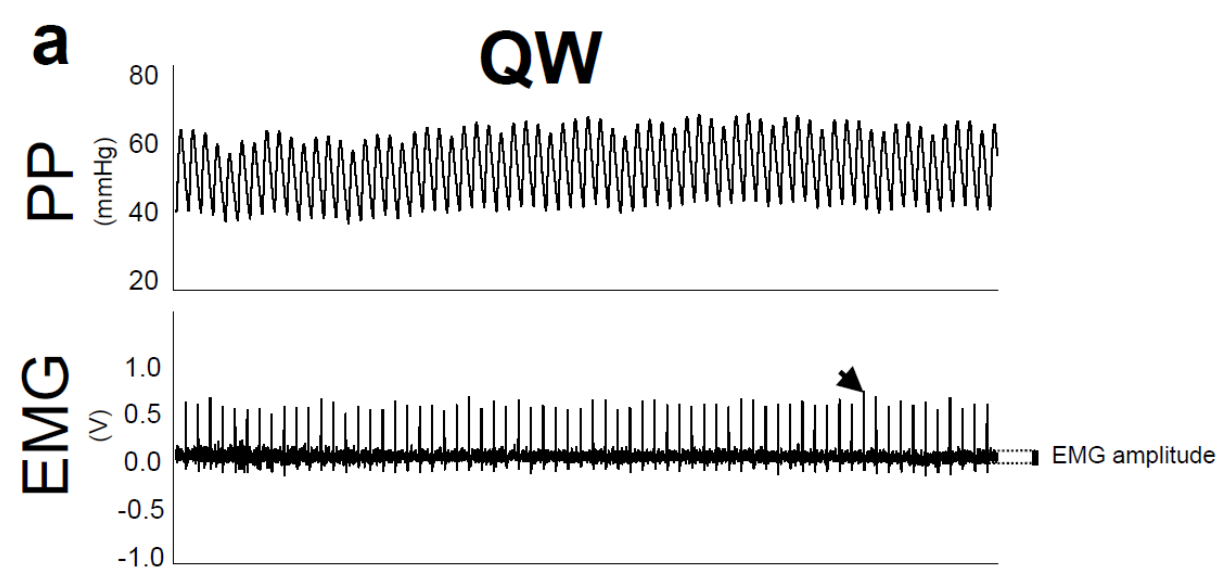

b
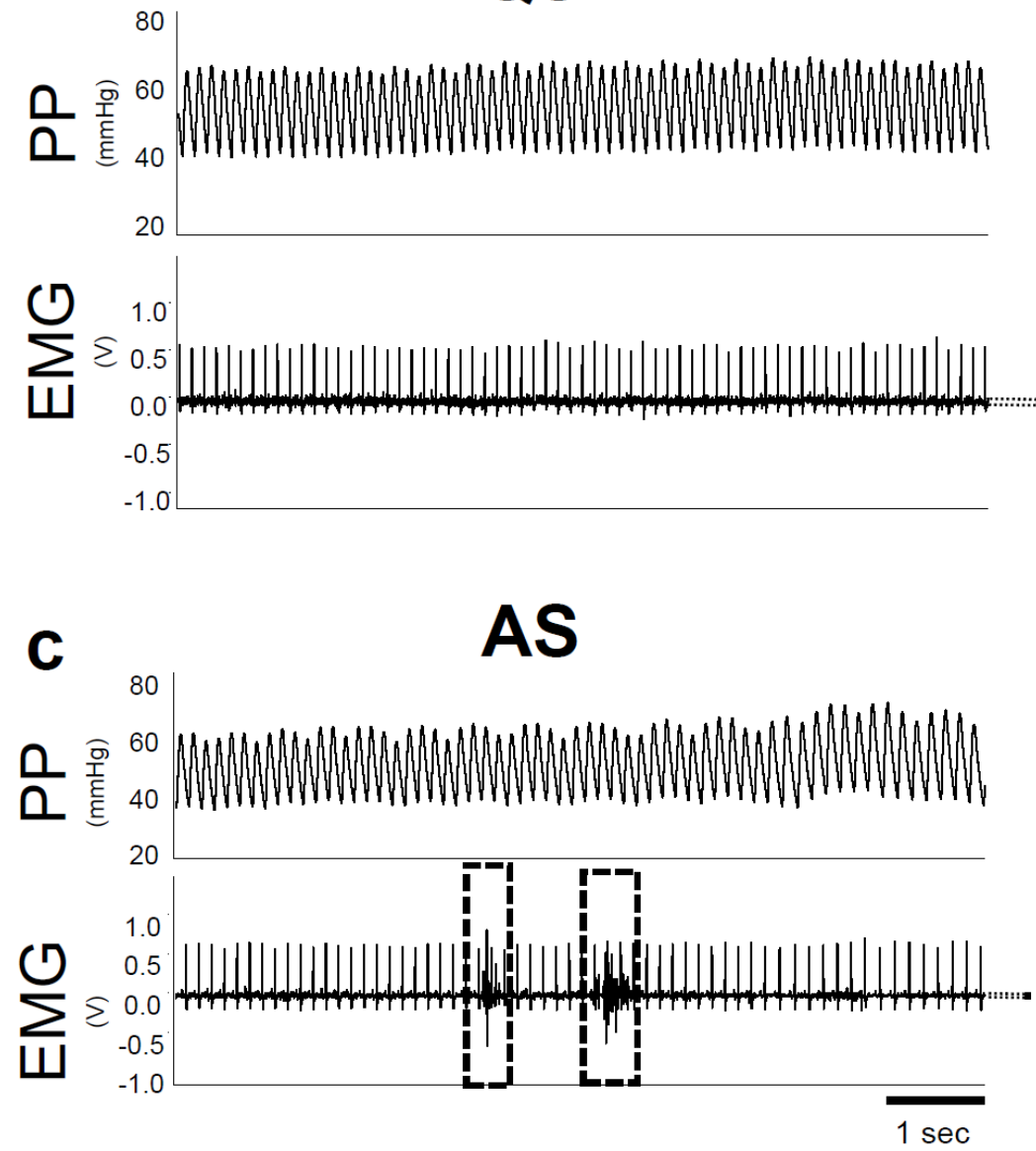

Figure 3.1. Arterial pulse pressure (PP) and nuchal electromyographic (EMG) records obtained from short periods of quiet wakefulness (QW, $a$ ), quiet sleep (QS, $b$ ), and active sleep (AS, $c$ ). Amplitude of nuchal EMG record (dark black section of trace) is indicated by dotted lines with the black vertical bar. Compared to QW, EMG amplitude is lower in QS, and is reduced further in AS. During AS, burst of activity associated with myoclonic twitching appear in the EMG record (dashed boxed regions). Nuchal EMG is superimposed on higher amplitude R-waves of the electrocardiographic record (indicated by arrowhead). 


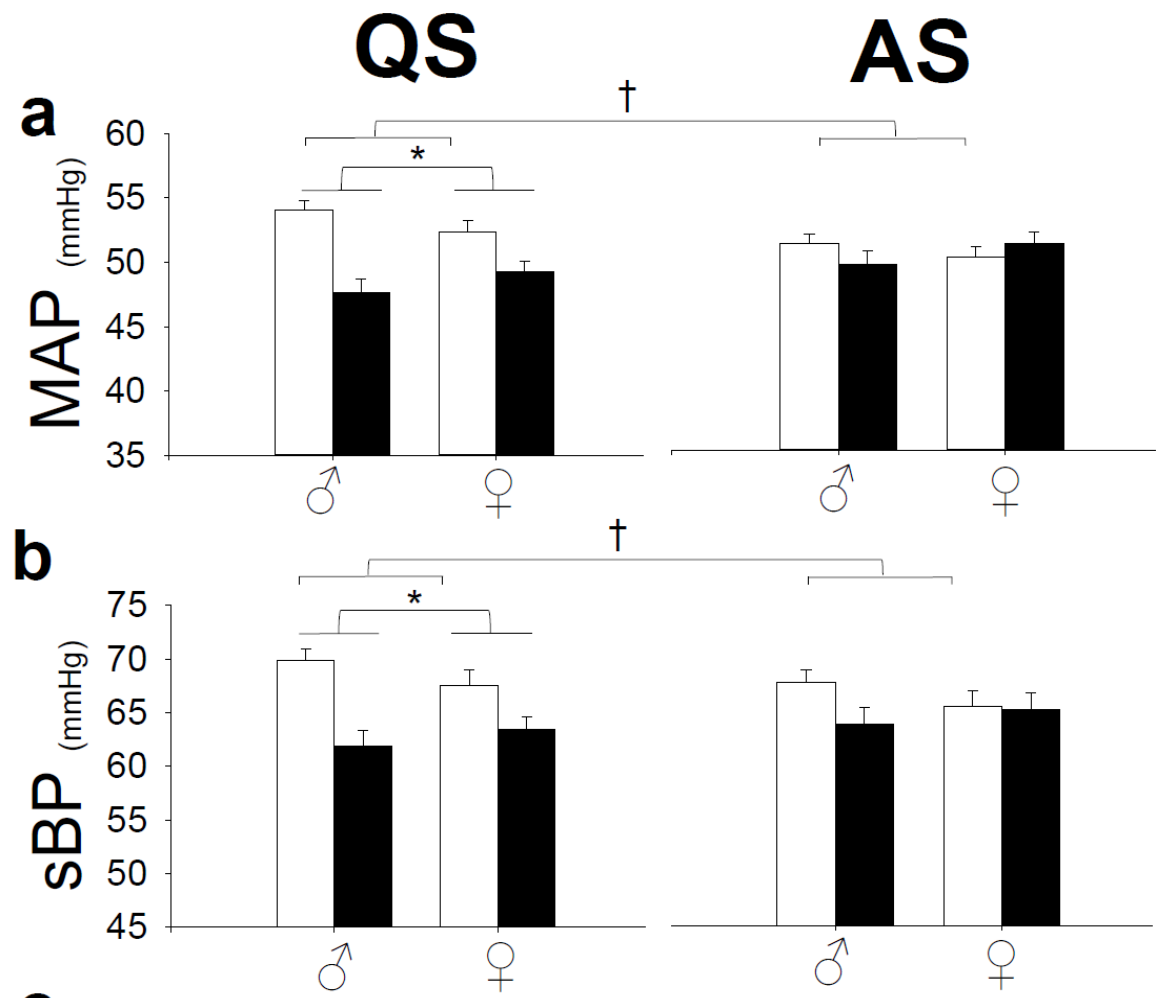

C
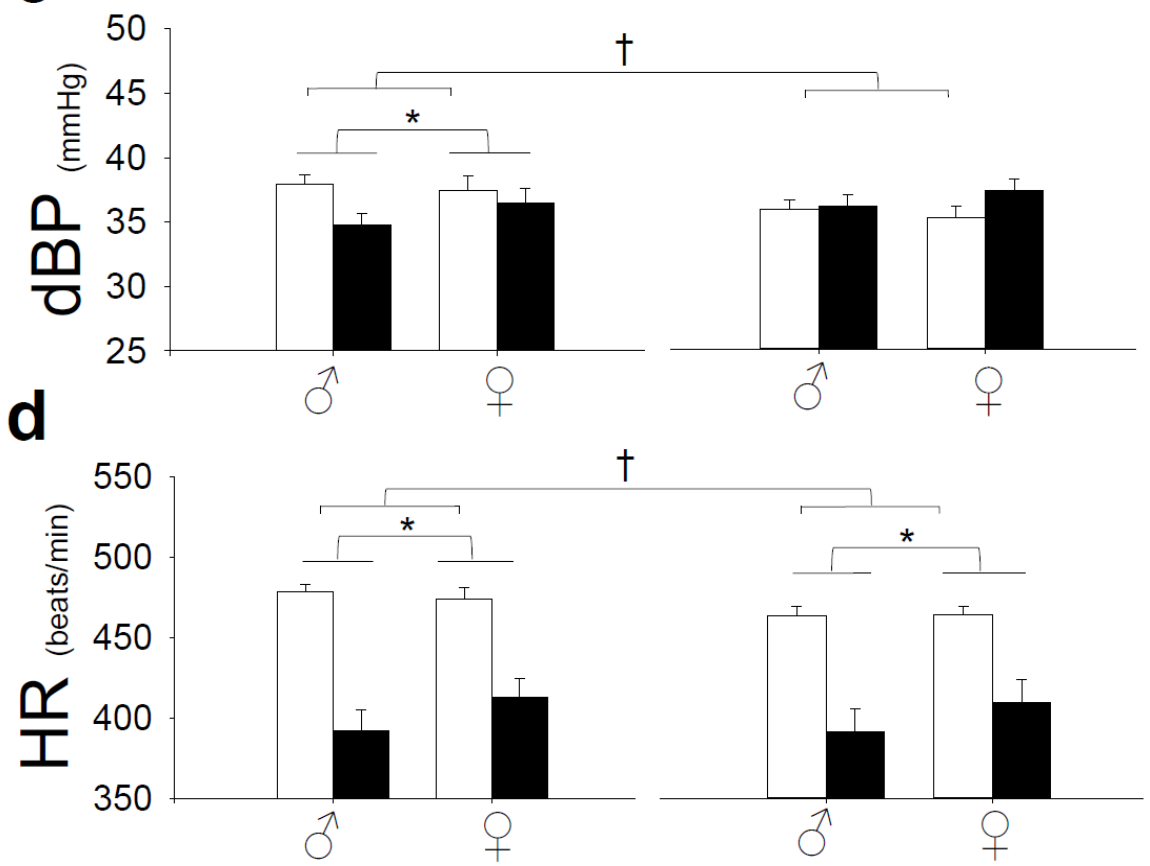

Figure 3.2. Central 5-HT deficiency leads to reduced blood pressure in quiet sleep, and reduced heart rate in both sleep states. Shown are: $a$ ) mean arterial pressure (MAP), b) systolic blood pressure (sBP), $c$ ) diastolic blood pressure (dBP), and $d$ ) heart rate (HR) for TPH2-/- rat pups (solid bars; $\mathrm{n}=34$ male, 35 female) and wildtype littermates (open bars; $\mathrm{n}=38$ male, 31 female) in quiet sleep (QS, left panels) and active sleep (AS, right panels). *: significant effect of genotype (2FA; $<<0.01$ for all variables). $\uparrow$ : significant interaction between genotype and sleep state, testing data from animals displaying both QS and AS (3FRMA; $<<0.001$ for all variables). For this and subsequent figures, data are mean values \pm standard error of the mean. 

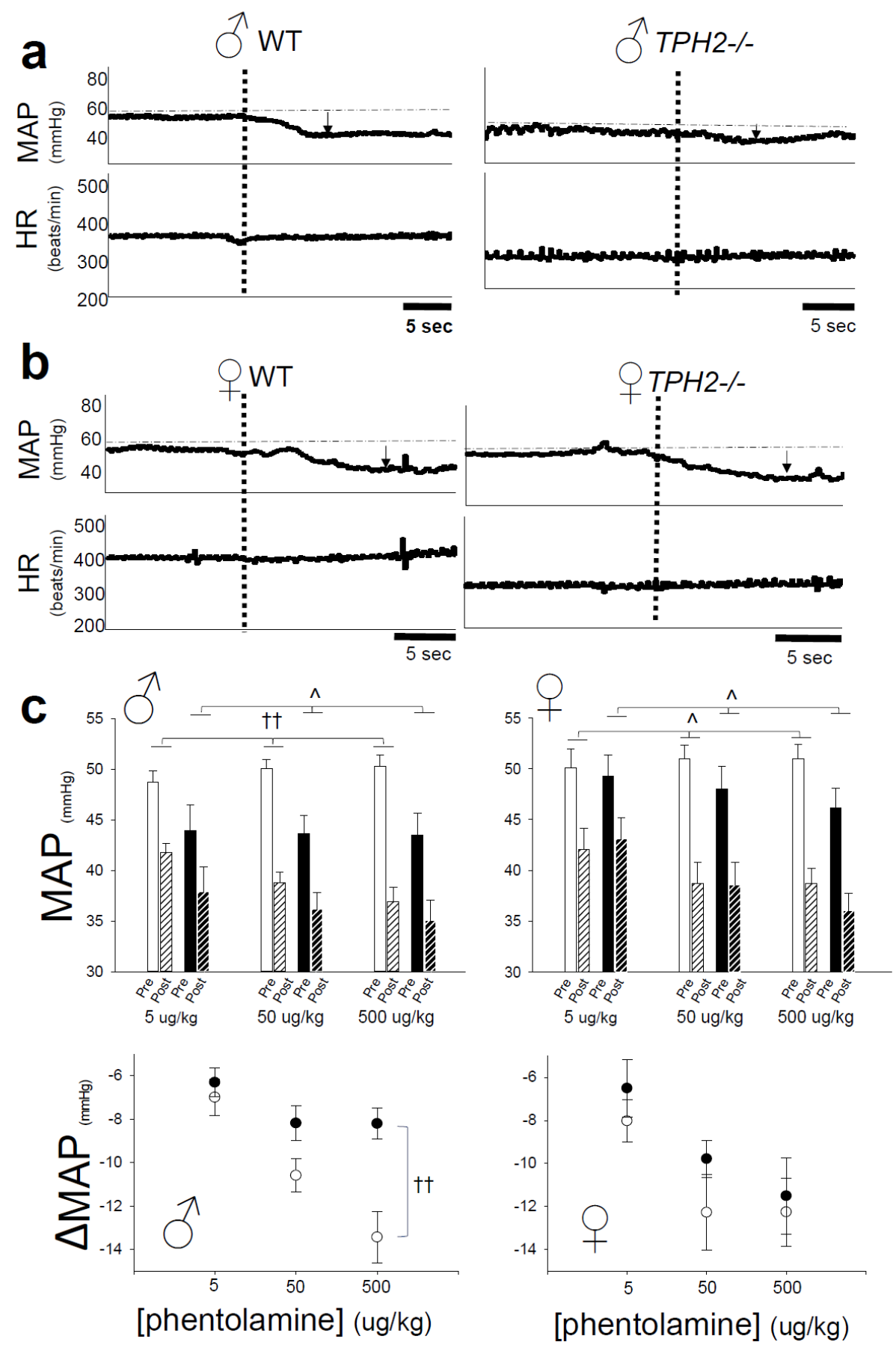

Figure 3.3. A loss of central 5-HT reduces sympathetic tone to the vasculature in males. Typical responses of mean arterial pressure (MAP) and heart rate (HR) to phentolamine for $a$ ) male wildtype (WT) and $T P H 2-/$ - pups and $b$ ) female WT and TPH2-/- pups. Note that phentolamine injection (at vertical dashed line) induced a smaller decrease in MAP in the male TPH2-/- pup compared to its WT counterpart, an effect not seen in females. $c$ ) top panels: average values for the MAP of WT (open bars, $\mathrm{n}=11$ males (left) and 6 females (right)) and TPH2-/-pups (closed bars; $\mathrm{n}=8$ males (left) and 7 females (right)) before (non-hatched bars) and after (hatched bars) i.v. phentolamine at 5,50 and $500 \mathrm{ug} / \mathrm{kg}$. ${ }^{\wedge}$ : significant effect of dose (3FRMA, $\mathrm{p}<0.001$ ); $\dagger \dagger$ : significant interaction between genotype, sex and dose on the fall in MAP (3FRMA: $p=0.04$ ). bottom panels: same data as in upper panels, expressed as the change in MAP from pre- to post-phentolamine treatment, in WT (open circles) and TPH2-/- pups (closed circles). 

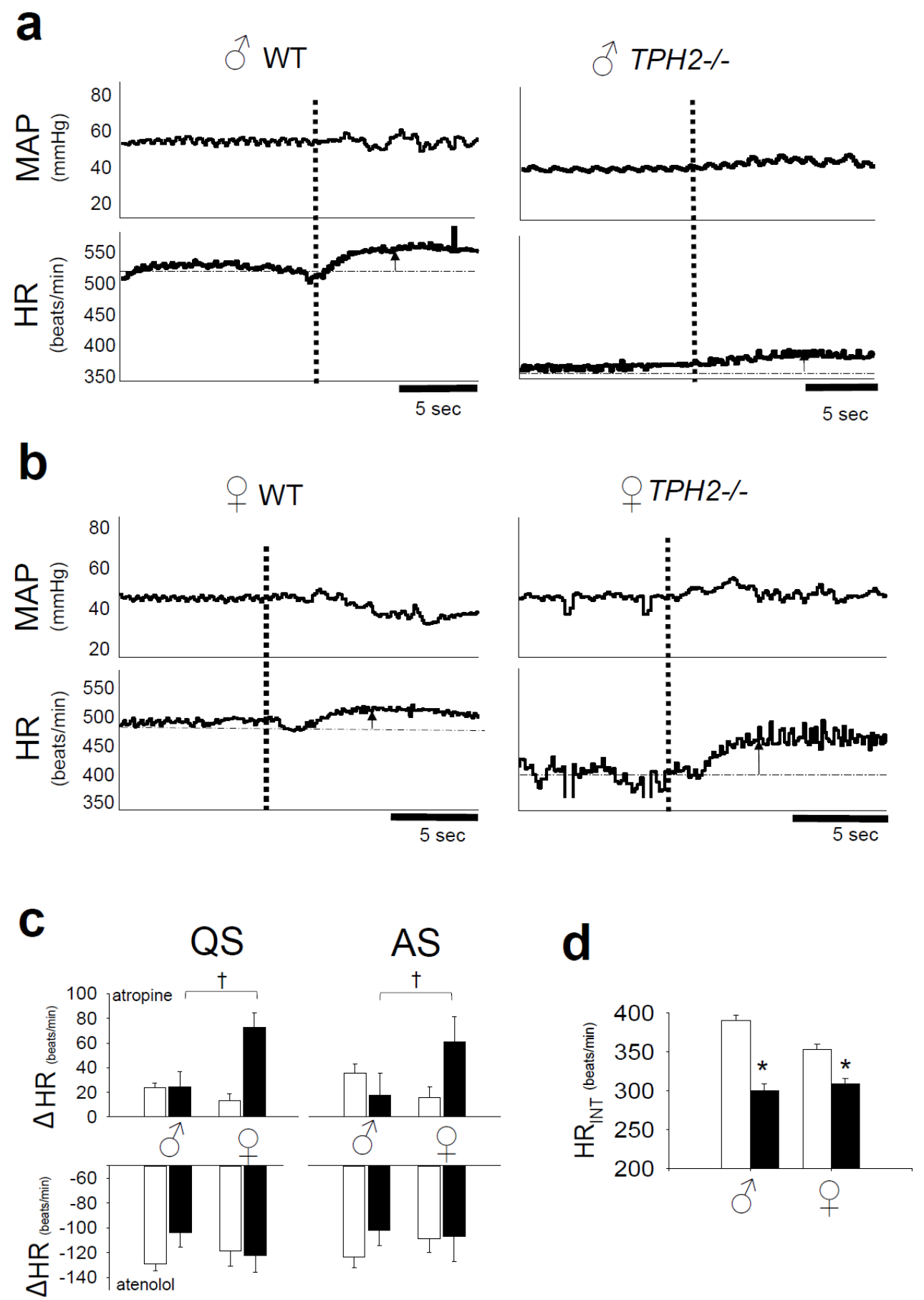

Figure 3.4. A loss of central 5-HT increases cardiac vagal tone in females. Typical responses of mean arterial pressure (MAP) and heart rate (HR) to atropine for $a$ ) male wildtype (WT) and TPH2-/- pups and $b$ ) female WT and TPH2-/- pups. Note that atropine injection (at vertical dashed line) induced a larger HR increase in the female TPH2-/- pup compared to its wildtype counterpart, an effect not seen in the male pups. c) change in HR in response to atropine (top panels) of WT (open bars; $\mathrm{n}=5$ male, 7 female) and TPH2-/pups (closed bars; $\mathrm{n}=6$ male, 6 female) and atenolol (bottom panels) (WT: $\mathrm{n}=8$ male, 6 female; TPH2-/-: $\mathrm{n}=10$ male, 10 female) in quiet (QS) and active sleep (AS). $\uparrow$ : significant genotype $\mathrm{x}$ sex interaction (2FA, $\mathrm{p}=0.016(\mathrm{QS})$ and $\mathrm{p}=0.028(\mathrm{AS}))$. d) Intrinsic HR ( $\left.\mathrm{HR}_{\mathrm{INT}}\right)$ of male and female WT (open bars) and TPH2-/pups (closed bars). *: significant effect of genotype $(2 \mathrm{FA}, \mathrm{p}<0.001)$. 

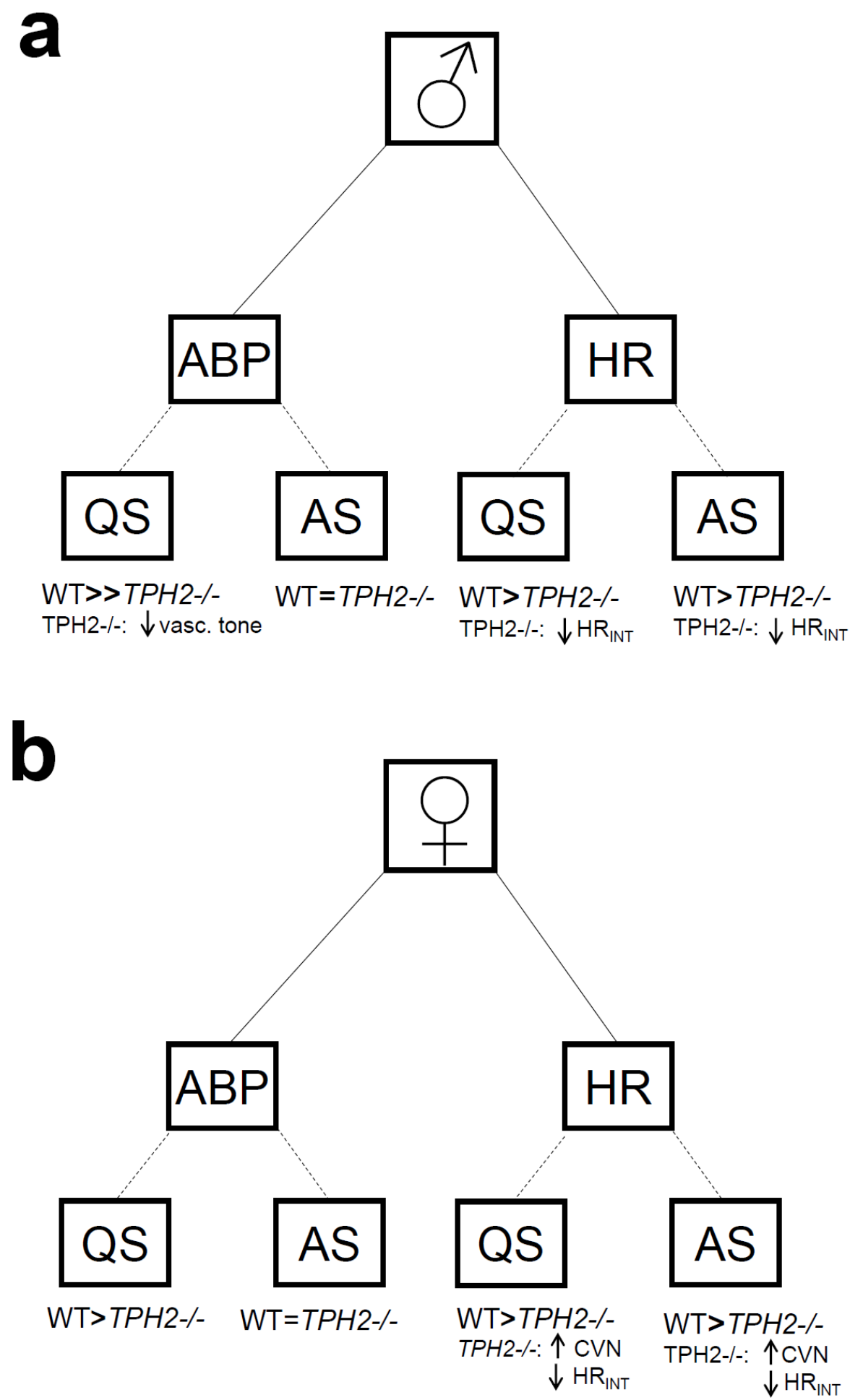

Figure 3.5. Summary of main findings. In both sexes, $T P H 2-/$ - pups have reduced arterial blood pressure (ABP) in quiet sleep (QS), but not in active sleep (AS). In males, but not females, central 5-HT helps maintain ABP by increasing sympathetic vascular tone. Both male and female TPH2-/- pups have reduced heart rate (HR) compared to wildtype littermates (WT), in part because of reduced intrinsic HR (HR $\mathrm{HNT}_{\mathrm{INT}}$ ). In both QS and AS, increased cardiac vagal nerve activity (CVN) contributes to the reduced HR of $\mathrm{TPH} 2-/$ - females. 


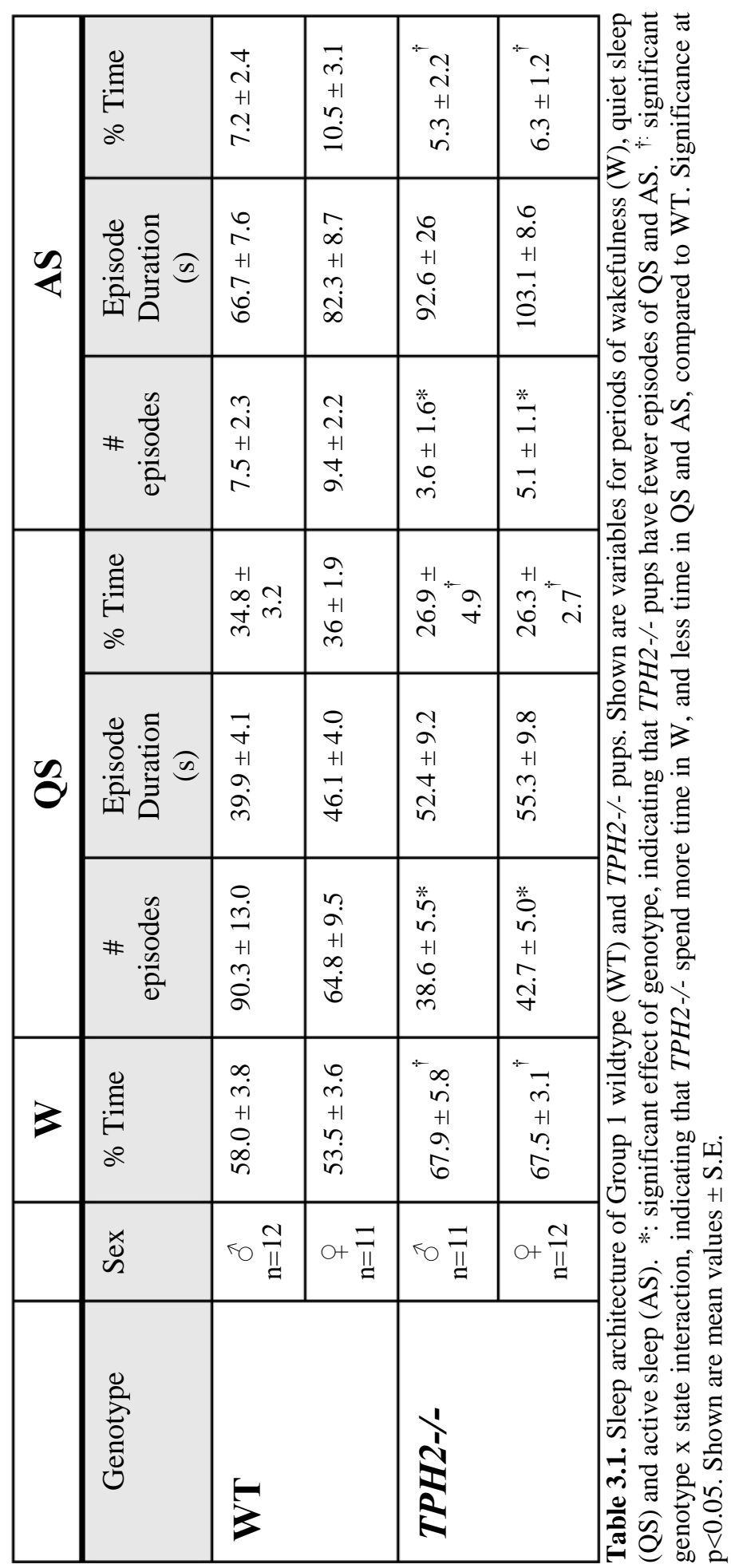




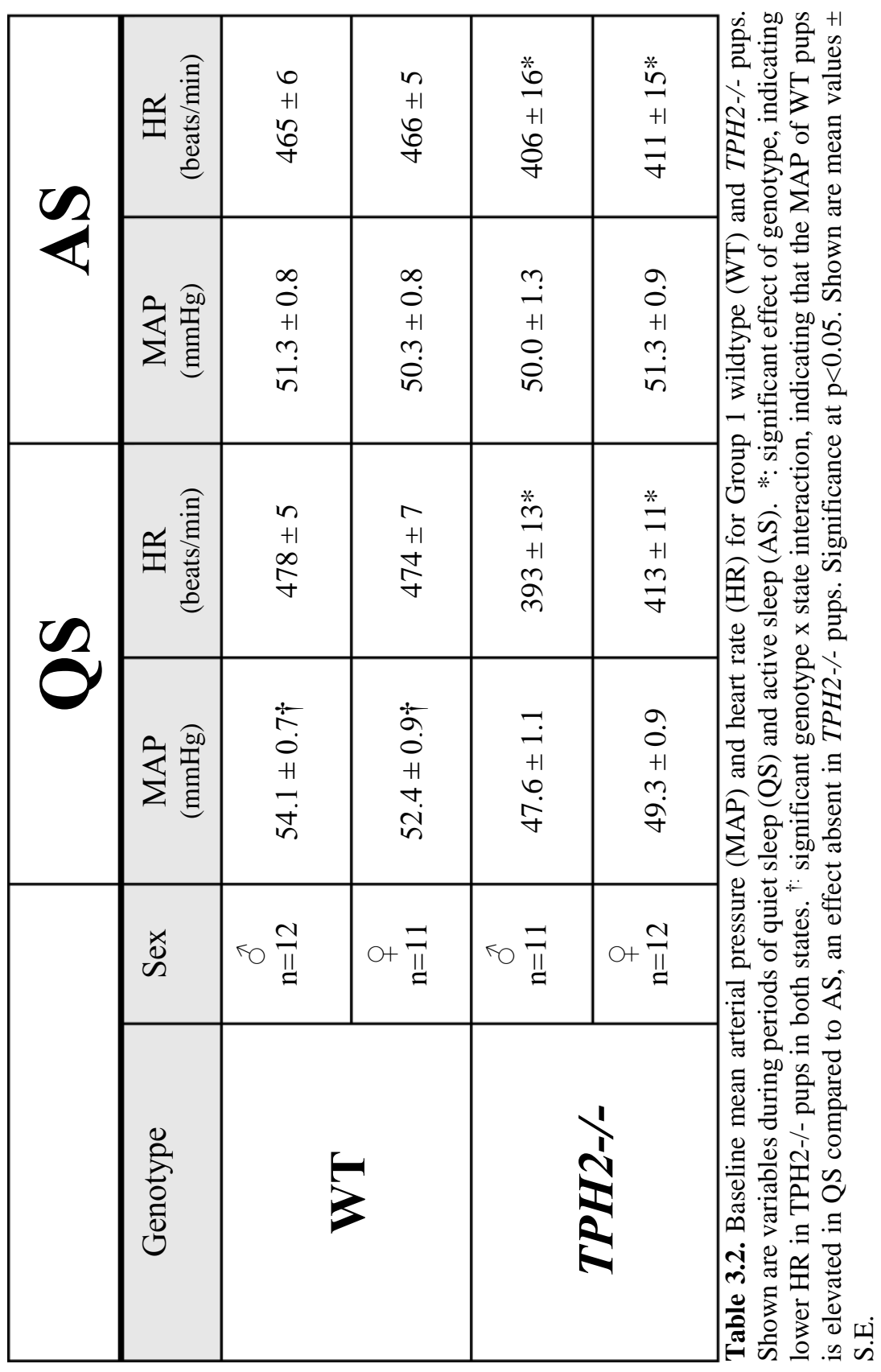


CHAPTER 4: Central serotonin prevents the development of hypertension in middleaged male rats: unravelling the mechanisms underlying a potentially novel form of neurogenic hypertension

Jennifer L. Magnusson and Kevin J. Cummings

Department of Biomedical Sciences, College of Veterinary Medicine, University of Missouri, Columbia, MO, USA 


\section{1 - ABSTRACT}

5-HT neurons within the midline raphe project to vagal and pre-sympathetic regions in the brain, yet the data from studies examining the role of central 5-HT on mean arterial blood pressure (MAP) regulation are conflicting or inconclusive. Given the hypotension presented in adult mice and infant rats lacking central 5-HT, and the fact that 5-HT neurons fire in a state-dependent manner, we hypothesized that in adulthood, central 5-HT would facilitate sympathetic vascular tone, thereby increasing MAP, and that this effect is most evident in wakefulness (QW) and non-rapid eye movement sleep (NREM), when 5-HT neurons are active, and less so during rapid eye movement (REM) sleep, when these neurons are silent. Given also that a sex-specific increase in MAP can occur with aging, we tested our hypotheses using both male and female tryptophan hydroxylase 2 knockout rats (TPH2-/-) that are deficient in central 5-HT, along with wild-type (WT) controls at 3 different ages: 2-3 month, 5-8 month, and 12-13 months. MAP and heart rate (HR) were measured during periods of QW, NREM, and REM sleep. In a subset of 5-8 month animals (TPH2-/- male $\mathrm{n}=11$, female $\mathrm{n}=9$; WT male $\mathrm{n}=11$, female $\mathrm{n}=8)$, we injected atropine methyl nitrate $(i . v ., 1 \mathrm{mg} / \mathrm{kg}$ ) and hexamethonium $(i . v ., 30 \mathrm{mg} / \mathrm{kg})$ to indirectly determine the extent to which central 5-HT contributes to sympathetic vascular tone. In a separate set of animals (5-8 months, TPH2-/- male n=6; WT male $\mathrm{n}=6)$, we injected losartan $(10 \mathrm{mg} / \mathrm{kg}$, i.v., an Angiotensin II (Ang II) type 1 receptor antagonist) to indirectly determine the degree to which Ang II contributes to resting MAP. Compared to WT, male TPH2-/- rats had increased MAP at 5-8 and 12-13 months of age, a phenotype not seen in female rats. Contrary to our hypothesis, the increased MAP in male TPH2-/- rats was evident across all states of vigilance. The fall in MAP following ganglionic and Ang II blockade was not 
influenced by a loss of 5-HT. These data suggest that a loss of central 5-HT is a factor that contributes to essential hypertension in men, but not women. While the mechanisms underlying 5-HT's effect on MAP remain unknown, our results suggest that 5-HT does not act through pathways that control either sympathetic nerve or systemic Ang II.

\section{2 - INTRODUCTION}

Hypertension of unknown origin (or essential hypertension (EH)) accounts for more than $90 \%$ of all hypertensive cases. Understanding its mechanisms is critical for the development of targeted therapies to not only lower blood pressure [242], but also lower its associated cardiovascular events (comorbidities), including stroke, myocardial infarction, and heart failure [241, 242]. The pathogenesis of hypertension may be linked to the pathophysiology of sleep. Non-rapid eye movement sleep is characterized by a marked reduction in mean arterial blood pressure (MAP), sympathetic activity, and heart rate (HR) [104], a phenotype that is absent or blunted in some hypertensive patients [243]. On the contrary, rapid eye movement sleep (REM) is marked by sympathetic activation as well as BP and HR instability [104]; spontaneously hypertensive rats exhibit marked BP surges during REM sleep [247]. There is also an increased incidence of REM sleep during the early morning period [246], as well as a peak incidence of cardiovascular events [244, 245].

Serotonin (5-Hydroxytryptamine, 5-HT) has been implicated in the pathophysiology of EH [233], presumably due to projections of 5-HT neurons within the midline raphe to vagal and presympathetic regions in the brain [199]; yet data from studies examining the role of central 5-HT on cardiovascular function is conflicting or inconclusive [199, 210, 295]. For instance, systemic depletion of 5-HT content elicits a 
significant rise in resting MAP in adult rodents [210]. However, neonatal rats treated centrally with 5,7-dihydroxytryptamine (5,7-DHT, a neurotoxin selective to 5-HT neurons) exhibit a reduced resting MAP and HR compared to saline injected controls [156]. However, 5-HT neurons contain other neuromodulators like thyrotropin releasing hormone and substance P [157], which can also contribute to cardiovascular control [296, 297]. Adult mice lacking central 5-HT have a reduced MAP, specifically in the early evening [224], suggesting that central 5-HT contributes to resting MAP. However, the activity of 5-HT neurons also depends on vigilance state, with maximal firing during quiet wakefulness (QW), reduced firing in NREM, and virtually no firing in REM sleep [223]. We have recently shown that neonatal rats lacking tryptophan hydroxylase 2 (TPH2), the rate limiting enzyme in the synthesis of central 5-HT, and thus lacking 5-HT in the CNS, have a reduced MAP during quiet sleep (QS, similar to NREM in adults), but not during active sleep (AS, similar to REM in adults) [298].

To our knowledge, the specific role of central 5-HT on cardiovascular function across states of vigilance in adult animals has not been studied. Based on our findings in neonatal rats [298], we originally hypothesized that central 5-HT prevents MAP from falling mostly during QW and NREM when 5-HT neurons are active. However, we found that by $\sim 6$ months of age, rats lacking central 5-HT exhibited an elevated MAP compared to WT rats, a phenotype that was present across all states, but was most obvious during REM sleep. Therefore, we sought to assess the mechanisms associated with this phenotype. As neurogenic hypertension is associated with elevated sympathetic drive [299] and/or elevated angiotensin II (Ang II) activity [29], we hypothesized that central 5-HT reduces sympathetic drive and/or circulating Ang II. 


\section{3 - METHODS}

\section{Ethical Approval}

The Institutional Animal Care and Use Committee at the University of Missouri at Columbia, MO. approved all experimental procedures.

\section{Animals and treatments}

We used 144 rats divided as follows: $2-3$ month TPH2-/- rats (male $\mathrm{n}=8$, female $\mathrm{n}=6$ ) and WT controls (male $\mathrm{n}=9$, female $\mathrm{n}=9$ ); 5-8 month $T P H 2-/$ - rats (male $\mathrm{n}=28$, female $\mathrm{n}=15$ ) and WT controls (male $\mathrm{n}=31$, female $\mathrm{n}=-12$ ); and 12-13 month $T P H 2-/-$ rats (male $n=6$, female $n=4$ ) and WT controls (male $n=9$, female $n=7$ ). Information on the generation of this rat model has been previously published [155]. Male and female TPH2-/- rats weighed the same as sex- and age-matched controls; except, at 5-8 months, where male TPH2-/- rats weighed significantly more than WT controls (TPH2-/-: 361g $\pm 6 \mathrm{~g}$; WT: $331 \mathrm{~g} \pm 5 \mathrm{~g} ; \mathrm{p}=0.01)$. Animals had unrestricted access to water and standard rat chow and were kept on a $12 \mathrm{hr}$ light-dark cycle. Unless otherwise indicated, all rodents received the same surgical procedures, described as follows.

\section{Assessment of sleep utilizing EEG/EMG in adult rodents}

Rats were surgically implanted with electroencephalography (EEG) and electromyography (EMG) electrodes (Plastics One Inc., Roanoke, VA) to assess vigilance state. Detailed surgical procedures have been described previously [251]. Briefly, sterile surgery was performed under isoflurane anesthesia; depth of anesthesia was assessed using hindlimb withdrawal. Rats were fixed in a stereotaxic frame for implantation of EEG and 
EMG electrodes. Two EMG electrodes were implanted into the dorsal neck muscles and three EEG electrodes were screwed into the skull, located as follows: the first electrode at $2 \mathrm{~mm}$ rostral to bregma and $2 \mathrm{~mm}$ lateral to the midline, the second electrode at $3 \mathrm{~mm}$ caudal to bregma and $2 \mathrm{~mm}$ lateral to the midline ipsilateral to the first screw, and a "ground" electrode was placed slightly between the two contralateral to midline and caudal to bregma. Following implantation, the EEG/EMG electrode wires were connected to a six-prong plastic pedestal (Plastics One Inc., Roanoke, VA). Dental acrylic (Lang Dental Manufacturing Co. Inc; Wheeling, IL) was used to secure the cap and electrodes to the skull. Following the surgery, animals were given Banamine $(2.5 \mathrm{mg} / \mathrm{kg}$; Zoetis Inc, Kalamazoo, MI) to prevent postoperative pain and Baytril $(5-10 \mathrm{mg} / \mathrm{kg}$; Bayer Healthcare, LLC; Shawnee Mission, KS) to help prevent bacterial infection. Animals were allowed a minimum of a 7-day recovery from EEG/EMG surgery prior to testing to ensure regular cycling of vigilance states. At least once during the week prior to testing, animals were acclimated to the recording chamber for 1-2 hours.

\section{Assessment of sleep-wake states}

Sleep-wake (vigilance) states were determined by EEG/EMG signal (Group 1) as well as visual inspection of behavior during the study (Groups 1-3). Vigilance states were classified as Active Wakefulness (not included in analysis), Quiet Wakefulness (QW), NREM and REM sleep. Active wakefulness was defined as desynchronized, low voltage, fast EEG with large amplitude EMG. During active wakefulness, the animal was eating, grooming or sniffing around the chamber. EEG patterns from QW are similar to Active Wakefulness, but EMG activity was stable. In QW, rats had their eyes wide open, and 
behaviorally, did not exhibit NREM characteristics. NREM was defined as a continuous, synchronized, low frequency $(0.5-4 \mathrm{~Hz})$ and high voltage EEG with an amplitude two to three times greater than observed in QW. REM sleep was characterized by desynchronized, low-voltage, high frequency EEG with the lowest observed EMG amplitude. We confirmed entry into REM sleep by observing myoclonic twitching of the distal muscles, a characteristic feature of REM sleep. Cardiorespiratory variables were assessed during each vigilance state. For each state, individual episodes greater than 10 s in duration were used for analysis. For each animal, average values for each variable were obtained across every state. These average values were used for statistical comparisons to assess the effects of genotype and state for each variable.

\section{Femoral Artery/Venous Catheterization}

Two days prior to testing, all adult animals were implanted with a femoral arterial catheter for the measurement of MAP, as we have described in a previous publication [156]. Groups 2 and 3 were also implanted with a femoral venous catheter for injection of drugs. Briefly, rats were anesthetized under $2 \%$ isoflurane and a $\sim 1 \mathrm{~cm}$ skin incision was made in the left groin. A 20X dissecting microscope was used to dissect out the left femoral artery. The artery was then tied with 5-0 surgical suture just distal to the epigastric branch. An incision was made on the left femoral artery for insertion of the PE10 portion of the inlab made catheter of PE10 fused to PE50 tubing (BD; Sparks, MD). Similar procedures were used for insertion of the femoral venous catheter. After catheters were secured in place, they were tunneled through the back with a small portion exteriorized between the shoulder blades to allow easy access for flushing daily with sterile heparinized $0.9 \%$ saline 
(100units/ml) until testing and sacrifice. Lidocaine was applied to the surgical sites postsurgery and both incision sites were sutured with sterile 5-0 surgical silk.

\section{Whole-body plethysmography}

We used whole body plethysmography to assess cardiorespiratory variables of interest during sleep, similar to previous studies in our lab [225, 298]. On the day of testing, rats were lightly anesthetized to reduce the stress associated with hooking up an EEG guidewire and extended catheter. Animals were then placed into the whole body plethysmography system for 7-8 hours, and we used data from the last 3-4 hours for analysis. This allowed for sufficient time for the animal to recover from light anesthesia, and establish normal MAP and HR and cycling between vigilance states. Animals were provided air from the wall, passed through a flowmeter prior to entering the chamber (flow $=1 \mathrm{~L} / \mathrm{min})$. Chamber pressure was maintained close to atmospheric by pulling air from the opposite end of the chamber via a wall vacuum. Changes in chamber pressure related to breathing were measured using a differential pressure transducer (Validyne), connected to the animal chamber on one side and an empty reference chamber on the other. The reference chamber was used to account for thermal drift and was connected to the test chamber via a $\sim 10 \mathrm{~cm}$ length of small-diameter tubing. Analog signals from transducers for both arterial pressure and respiration were fed into a Powerlab data acquisition system (ADInstruments) and analysed in LabChart 7.3.7 (ADInstruments).

\section{Experimental Groups and Protocols}


Experiments were performed during the "light period" (8am-5pm) on three groups of animals. For all groups, animals were allowed to recover from light anaesthesia (used to setup an extended catheter tubing and EEG/EMG guidewire) for four hours. Group 1 animals (2-3 month $T P H 2-/$ - rats (male $\mathrm{n}=8$, female $\mathrm{n}=6$ ) and WT controls (male $\mathrm{n}=9$, female $\mathrm{n}=9$ ); 5-8 month $T P H 2-/$ - rats (male $\mathrm{n}=9$, female $\mathrm{n}=6$ ) and WT controls (male $\mathrm{n}=12$, female $\mathrm{n}=4$ ); and 12-13 month $\mathrm{TPH} 2-/$ - rats (male $\mathrm{n}=6$, female $\mathrm{n}=4$ ) and WT controls (male $\mathrm{n}=9$, female $\mathrm{n}=7)$ ) were then left unperturbed for an additional four hours beyond the initial acclimation period of 4 hours (total time in chamber: $\sim 8 \mathrm{hrs}$ ) and were solely used to investigate the extent to which central 5-HT deficiency affected MAP and HR during QW, NREM and REM sleep. Baseline (pre-drug) periods from animals in Groups 2 and 3 were pooled with animals in Group 1 as this period was statistically similar among groups.

Group 2 rats (5-8 month, $T P H 2-/-$ male $n=11$, female $n=9$; WT male $n=11$, female $\mathrm{n}=8$ ) were used to assess the effects of central 5-HT deficiency on baseline parasympathetic and sympathetic tone to the heart and vasculature. Following the initial four-hour acclimatization period, animals were left unperturbed for an additional 2.5-3 hours to assess resting MAP and HR during QW, NREM and REM sleep. We then injected atropine methyl nitrate $(1 \mathrm{mg} / \mathrm{kg}$, i.v., Sigma Chemical) to block parasympathetic tone followed by hexamethonium (30mg/kg, Sigma Chemical) to induce ganglionic blockade and allow for the quantification of the sympathetic contribution to ABP. As animals were pre-treated with atropine, we also measured the acute HR response to hexamethonium as a reflection of sympathetic drive to the heart in both $T P H 2-/$ rats and WT controls. In our initial experiments, we administered losartan after ganglionic blockade. However, in this small group of rats (TPH2-/- male $\mathrm{n}=6$; WT male $\mathrm{n}=6$ ), baseline ABP was not different in $T P H 2$ - 
/- rats compared to WT controls. Further, the MAP in the presence of ganglionic blockade was the same in both genotypes. Because of this, we were unable to confidently interpret the MAP data following the application of losartan in the absence of autonomics.

Group 3 rats were used to further address the mechanism behind the MAP phenotype in 5-8 month old rats, addressing the hypothesis that elevated angiotensin II may contribute to the elevated basal MAP in central 5-HT deficiency. Following the initial fourhour acclimatization period, animals were left unperturbed for an additional 2.5-3 hours to assess resting MAP and HR during QW, NREM and REM. We then injected losartan (a specific AT1 receptor blocker; 10mg/kg, i.v. Sigma Chemical; 5-8 months, TPH2-/- male $\mathrm{n}=6$; WT male $\mathrm{n}=6$ ) to block angiotensin signalling both centrally and peripherally followed by injection of atropine methyl nitrate and hexamethonium to block autonomic drive, similar to Group 2 rats.

\section{Data and Statistical Analysis}

In all animals, we measured the following from raw respiratory and BP traces: MAP $(\mathrm{mmHg})$, systolic arterial blood pressure $(\mathrm{sBP}, \mathrm{mmHg})$, diastolic arterial blood pressure (dBP, mmHg), HR (beats/min), respiratory frequency (fB; breaths/min), tidal volume $\left(\mathrm{V}_{\mathrm{T}}\right.$, $\mathrm{ml} / \mathrm{kg}$ ), ventilation $\left(\mathrm{V}_{\mathrm{E}}, \mathrm{fB} \times \mathrm{V}_{\mathrm{T}} ; \mathrm{ml} / \mathrm{min} / \mathrm{kg}\right.$ ), and the coefficient of respiratory period variability (CVP\%) - a measure of respiratory stability. In a subset of animals, we also measured metabolic rate $\left(\mathrm{VO}_{2}, \mathrm{ml} / \mathrm{min} / \mathrm{kg}\right)$ and the metabolic equivalent $\left(\mathrm{V}_{\mathrm{E}} / \mathrm{VO}_{2}\right)$. We also assessed the frequency of the spectral bands for very low (vLF, $0-0.2 \mathrm{~Hz}, \mathrm{mmHg}^{2}$ ) and low ( $\mathrm{LF}, 0.2-.075 \mathrm{~Hz}, \mathrm{mmHg}^{2}$ ) frequency components of systolic blood pressure variability. The vLF component reflects changes in sympathetic outflow related to 
hormonal activity or thermoregulation while the LF component reflects sympathetic nerve activity [300, 301].

For each animal, we averaged each variable during each sleep state (e.g. MAP during NREM; see tables 4.1 and 4.2 for the average number of times male and female rats of each age exhibited each sleep state). Graphically, all data presented are from the raw data. For statistical purposes, we performed a log transformation (IBM SPSS v25 software, Armonk, NY) on all data variables as most were non-normally distributed. Significant differences on all variables were assessed using a student's t-test, 2-Factor ANOVA, or a 2-Way RM ANOVA (2WRMA) using SigmaPlot 14 (Systat Software Inc, USA) or a 3Way RM ANOVA (3WRMA, IBM SPSS software, Armonk, NY) followed by Tukey’s post hoc analysis.

\section{4-RESULTS}

Sleep-architecture during baseline conditions

During the baseline period ( $\sim 3$ hours) that followed the initial acclimatization period, we analyzed the number of QW, NREM, and REM episodes, the duration of these episodes, as well as the total percent time spent in each vigilance state in male (Table 4.1) and female (Table 4.2) TPH2-/- rats and WT controls. 2-3 month-old male TPH2-/- rats had longer episodes of QW, NREM, and REM compared to WT controls (2WRMA, genotype $\mathrm{p}=0.003$ ). We also observed this sleep phenotype in female $T P H 2-/$ - rats, except for NREM duration, which was unaffected by central 5-HT deficiency (2WRMA, genotype $\mathrm{p}<0.001)$. Despite their longer REM episodes (2WRMA, genotype*vigilance-state $\mathrm{p}=0.001$ ), overall $\mathrm{TPH} 2-/$ - rats spent the same amount of time in each vigilance-state as 
sex-matched WT controls (expressed as \% total time; 2WRMA, genotype p>0.05), due to a reduced number of REM episodes in TPH2-/- rats (2WRMA, genotype*vigilance-state interaction $\mathrm{p}=0.021$ ).

In 5-8 month old male and female rats, the duration of NREM and REM, but not QW, episodes were longer in TPH2-/- compared to sex-matched WT controls (2WRMA, genotype $\mathrm{p}<0.001)$. Regardless, similar to younger rats, the overall percent time spent in each vigilance-state was not different between male and female TPH2-/- and sex-matched WT controls (2WRMA, genotype $\mathrm{p}>0.05$ ), again because of reduced numbers of REM and NREM episodes in TPH2-/- animals (2WRMA, genotype $\mathrm{p}<0.001)$. Male $T P H 2-/-$ rats spent less time, as a percentage, in QW compared to female TPH2-/- rats (3WRMA, genotype*vigilance-state*sex $\mathrm{p}=0.017)$, in part because they had fewer $\mathrm{QW}$ episodes (3WRMA, genotype*vigilance-state*sex $\mathrm{p}=0.048$ ). Female $T P H 2-/-$ rats spent less overall percent time in REM compared to male TPH2-/- rats (3WRMA, genotype*vigilancestate*sex $\mathrm{p}=0.017)$.

The duration of QW, NREM, and REM episodes was greater in 12-13 month-old male and female $T P H 2-/-$ rats compared WT counterparts (2WRMA, genotype p<0.001). However, as we observed in younger animals, the percent time that male and female TPH2/- rats spent in NREM and REM sleep were the same as controls, given that they had fewer NREM and REM episodes (2WRMA, genotype*vigilance-state $\mathrm{p}<0.001)$. The percent time that male $T P H 2-/-$ rats spent in QW was greater than WT counterparts (2WRMA, genotype* vigilance-state $\mathrm{p}=0.039)$.

In summary, central 5-HT maintains wakefulness and inhibits REM sleep generation, as evidenced by elongated REM episodes in rats lacking central 5-HT. 
Central 5-HT deficiency increases arterial blood pressure across all vigilance states in adult and middle-aged rats

We hypothesized that central 5-HT deficiency would reduce MAP, similar to previous reports in $T P H 2-/$ - adult mice [224], especially in QW and NREM when 5-HT neurons are active [223], similar to what we reported recently in TPH2-/- rat pups [298]. Given that MAP changes with age, and in a sex-specific manner, we tested this hypothesis at three ages: 2-3, 5-8, and 12-13 months. MAP data are shown in Figure 4.2; HR data are shown in Figure 4.3, and all other cardiovascular variables are shown in Tables 4.3 (males) and 4.4 (females).

At 2-3 months of age, there were no significant differences in MAP between male (Fig. 4.2a, left) or female (Fig. 4.2a, right) $T P H 2-/-$ rats and sex-matched WT controls (2WRMA, p>0.05). For both genotypes, there was a significant effect of vigilance-state on MAP and HR, irrespective of sex. MAP was greatest during REM (2WRMA, vigilancestate interaction $\mathrm{p}<0.001$, Fig. 4.2a), due to an elevated $\mathrm{sBP}$ and dBP during REM sleep (2WRMA, vigilance-state $\mathrm{p}<0.001)$.

In contrast to 2-3 month-old rats, at 5-8 months of age, both male and female TPH2/- rats had significantly increased MAP compared to WT controls, irrespective of vigilancestate (3WRMA, genotype $\mathrm{p}=0.001$, Fig. $4.2 \mathrm{~b}$ ). Although there was no statistical interaction between sex and central 5-HT deficiency on MAP regulation, the lack of central 5-HT appeared to be most profound in males (compare male vs female MAP in Fig. 4.2b). Both sBP and dBP were elevated in $T P H 2-/-$ rats compared to WT controls; however, dBP reached statistical significance only in QW and REM (3WRMA, genotype*vigilancestate*sex $\mathrm{p}=0.001$ ). In all rats (i.e. irrespective of genotype and sex), blood pressure 
variables were higher in REM sleep compared to NREM or QW (2WRMA, vigilance-state $\mathrm{p}<0.001)$.

At 12-13 months of age, MAP was elevated in male TPH2-/- compared to WT controls irrespective of vigilance state (2WRMA, genotype $\mathrm{p}=0.021$, Fig. $4.2 \mathrm{c}$, left); this phenotype was not observed in female TPH2-/- rats compared to WT controls (2WRMA, genotype p>0.05, Fig. 4.2c, right). In contrast to their MAP phenotype at 5-8 months of age, 12-13 month-old male $T P H 2-/$ rats had an elevated dBP, but not sBP, compared to WT controls (2WRMA, genotype $\mathrm{p}=0.008$ ). As was the case in 5-8 month-old rats, blood pressure variables in 12-13 month old rats were higher in REM sleep compared to NREM and QW, irrespective of genotype or sex (2WRMA, vigilance-state $\mathrm{p}<0.001$, Fig. 4.2c). Age did not appear to significantly affect ABP (e.g. compare Figure 4.2 a, b, and c).

In summary, by 5-8 months of age, central 5-HT is necessary to prevent MAP from increasing, a function independent of vigilance-state. Further, while female $T P H 2-/$ - rats had an elevated $\mathrm{ABP}$ at 5-8 months of age, by 12-13 months of age, female rats were protected from this phenotype.

Central 5-HT deficiency does not influence HR in adult rats

In 2-3 month old male rats, there were no significant influence of genotype on HR in any vigilance state (2WRMA, p>0.05, Fig. 4.3a, left). In female TPH2-/- rats, HR was greater in QW compared to NREM, but in WT controls, HR was greater in QW compared to REM sleep (2WRMA, genotype*vigilance-state interaction $\mathrm{p}=0.03$, Fig. $4.3 \mathrm{a}$, right), an effect of 5-HT deficiency that was not observed in male rats. 
The HR of 5-8 month-old TPH2-/- males was lower in NREM and higher in REM compared to WT controls (2WRMA, genotype*vigilance-sate $\mathrm{p}<0.001$, Fig. 4.3b, left). This phenotype was absent in female $T P H 2-/-$ rats (2WRMA, genotype p $>0.05$, Fig $4.3 b$, right). Irrespective of genotype or sex, HR was highest during QW (2WRMA, vigilancestate $\mathrm{p}<0.001$, Fig. $4.3 \mathrm{~b}$ ). HR was lower in male rats compared to female rats (3WRMA, sex, $\mathrm{p}<0.001$, Fig. 4.3b).

The HR of 12-13 month-old TPH2-/- male rats was higher during REM (2WRMA, genotype*vigilance-state $p=0.038$, Fig. $4.3 \mathrm{c}$, left). This was due to the fact that, in male WT rats, HR decreased across transitions from QW to NREM to REM, while in male TPH2-/- rats, HR increased across transitions from QW to REM (2WRMA, genotype*vigilance-state $\mathrm{p}=0.002$, Fig. $4.3 \mathrm{c}$, left). In female rats, there was no significant influence of genotype on HR (2WRMA, genotype p>0.05, Fig. 4.3c, right). Similar to 5-8 month old rats, HR was lower in 12-13 month old male rats compared to females (3WRMA, sex, p<0.001, Fig. 4.3c).

In summary, central 5-HT does not contribute to resting HR, irrespective of vigilance-state. Further, by 5-8 months, HR was lower in male rats compared to female rats.

Evidence that central 5-HT deficiency influences sympathetic vascular tone

To start addressing the physiological underpinnings of the high MAP in rats deficient in central 5-HT, we performed a power spectral analysis of sBP variability, analyzing the percentages of very low (vLF\%) and low (LF\%) frequency components of total sBP variability as an indirect indicator of sympathetic tone to the vasculature [300, 
301]. The vLF component reflects changes in sympathetic outflow related to hormonal activity, thermoregulation or blood flow to meet local metabolic demands while the LF component reflects sympathetic drive to the vasculature $[300,301]$. These data are presented in Tables 4.3 and 4.4. In 2-3 month-old male and female rats, vLF\% and LF\% were not influenced by genotype or vigilance state (2WRMA, p>0.05). In 5-8 month-old rats, vLF\% was greater in male $T P H 2-/-$ compared to WT controls (2WRMA, $\mathrm{p}=0.043$ ) but in female rats, vLF\% was not influenced by genotype (2WRMA, p>0.05). Likewise, LF\% was greater in male TPH2-/- compared to WT controls (2WRMA, genotype $\mathrm{p}<0.001$ ). LF\% was highest during QW irrespective of genotype (2WRMA, vigilance-state $\mathrm{p}<0.001)$ and was lower in males compared to females (3WRMA, sex, $\mathrm{p}<0.001$ ). In 12-13 month old male rats, there was no significant effect of genotype on vLF\% and LF\%, although TPH2/- tended to have higher values (2WRMA, $\mathrm{p}=0.058$ ). vLF\% and LF\% were greatest during REM irrespective of genotype and sex $(2 \mathrm{WRMA}$, vigilance-state $\mathrm{p}<0.001)$ and were lower in male compared to female rats (3WRMA, sex, $\mathrm{p}=0.043)$.

In summary, by 5-8 months of age, central 5-HT appears to be necessary in preventing an increase in sympathetic vascular tone, which may contribute to the elevated ABP we found in central 5-HT deficiency. However, by 12-13 months of age, 5-HT does not appear to affect sympathetic vascular tone. Therefore, other mechanisms may be contributing to the elevated MAP in central 5-HT deficiency at this age.

Altered autonomic drive to the heart or vasculature does not contribute to the elevated $A B P$ in adult male rats 
Elevated sympathetic drive can contribute to hypertension [299]. To assess the extent to which increased sympathetic vascular tone contributed to the increased MAP of TPH2-/- rats compared to WT, we treated 5-8 month-old TPH2-/- and WT controls with atropine methyl nitrate (i.v., $1 \mathrm{mg} / \mathrm{kg}$ ) and hexamethonium (i.v., $30 \mathrm{mg} / \mathrm{kg}$ ) to block parasympathetic and sympathetic drive, respectively. We measured the subsequent drop in MAP (following hexamethonium) as an indicator of sympathetic drive to the vasculature (Fig. 4.4a). Average absolute responses are shown in male (Fig. 4.4b, left) and female (Fig. $4.4 \mathrm{~b}$, right) rats. The rise in MAP following atropine (Atrop, 2WRMA, $\mathrm{p}>0.05$ ) and the fall in MAP following hexamethonium (Hex, 2WRMA, p>0.05) were not influenced by genotype or sex (Fig. 4.4b). In fact, when expressed as $\triangle \mathrm{MAP}$, if anything, TPH2-/- rats have a reduced fall in MAP compared to WT (although this is not significantly different than WT) (Fig. 4.4c). Thus, compared to WT, MAP was still elevated in male TPH2-/- rats following ganglionic blockade. Figure 4.5 shows the raw MAP as a time control for our experiments, showing MAP remained elevated in TPH2-/- rats compared to WT controls (2WRMA, genotype $\mathrm{p}=0.039$ ) following the immediate drop in MAP and compensatory rise in MAP following ganglionic blockade. We measured these time-points at roughly the time in the experiment that AT1 (AT1x TC) and V1A (V1Ax TC) blockade would have occurred in Group 2 animals.

In summary, at 5-8 months of age, central 5-HT does not contribute to autonomic tone to the heart or vasculature, suggesting that elevated sympathetic tone does not contribute to the MAP phenotype in central 5-HT deficiency.

Angiotensin II does not contribute to the elevated ABP in adult male rats 
To assess whether elevated angiotensin II (Ang II) signalling could be contributing to the elevated MAP of $T P H 2-/$ - rats, we utilized losartan, an Angiotensin type 1 receptor type antagonist (AT1x), to test the hypothesis that there is greater Ang II signalling in 5-8 month old male rats devoid of CNS 5-HT. In our initial experiments, we administered losartan after ganglionic blockade (Fig. 4.6). First, we noticed that in this subset of rats, MAP was not significantly different at rest in TPH2-/- compared to WT controls. Second, the response to ganglionic blockade was not different between genotypes (Fig. 4.6b), a finding inconsistent with our original dataset (Fig. 4.4b). Consequently, we were unable to accurately interpret the responses to AT1x and V1Ax in this dataset. We found that, compared to animals treated solely with ganglionic blockade (Fig. 4.5b, AT1x TC and V1Ax TC), the MAP in both TPH2-/- rats and WT controls was lower following ganglionic blockade + AT1x + V1Ax (Fig. 4.6b). This data suggests that Ang II and/or AVP may contribute to the compensation that occurs following the significant drop in MAP induced by ganglionic blockade. However, more animals are required to 1) address the lack of phenotype in $\mathrm{TPH} 2-/-$ rats compared to WT controls at rest and 2) following ganglionic blockade, assess whether in central 5-HT deficiency, Ang II or AVP contribute to vascular tone in the absence of autonomics.

Therefore, we decided to inject losartan in the absence of complete ganglionic blockade (atropine + hexamethonium) in order to avoid the confounding influence of neurohumoral compensation [302], thus allowing us to directly assess whether elevated Ang II signalling (be it central or peripheral) contributes to elevated MAP of TPH2-/- (Fig. 4.7). Following losartan treatment, the MAP of male $T P H 2-/-$ rats was still elevated compared to WT controls (AT1x, 2WRMA, p=0.034, Fig. 4.7b) suggesting that elevated 
Ang II signalling does not contribute to the elevated ABP in male TPH2-/- rats. In other words, the change in MAP ( $\triangle \mathrm{MAP})$ in response to losartan was not influenced by genotype (t-test, $\mathrm{p}>0.05$, Fig. 4.7c). At the end of these experiments, we assessed whether ganglionic blockade was complete by injecting phenylephrine (PE, i.v. $10 \mu \mathrm{g} / \mathrm{kg}$, Supplemental Fig. 4.11). We found that, at the time of injection, there was a small but significant drop in HR in response to the PE induced rise in MAP.

In summary, at 5-8 months of age, central 5-HT does not affect Ang II signalling, suggesting that elevated Ang II does not contribute to the ABP phenotype in central 5-HT deficiency.

Spontaneous hypertensive events do not contribute to the elevated ABP during REM sleep in male TPH2-/- rats

In both WT and TPH2-/- rats, we observed acute, severe increases in MAP (i.e. extreme hypertensive events (EHE's) during REM sleep, Figure 4.8). For both genotypes, we quantified the number and magnitude of these spikes to determine if they contributed to the elevated MAP in male and female TPH2-/-rats. EHE's were defined as any elevation in MAP that was at least $20 \mathrm{mmHg}$ above the average MAP during NREM sleep for that animal. In 2-3 month-old rats, the number of EHE's during REM was not different between TPH2-/- and WT controls (2-Factor ANOVA, p>0.05, Fig. 4.8b). By 5-8 months of age, both male and female $T P H 2-/-$ rats had significantly more EHE's than sex-matched WT controls (2-Factor ANOVA, p<0.004, Fig. 4.8c). However, by 12 months of age, both male and female TPH2-/- rats had the same number of EHE's as WT controls (2-Factor 
ANOVA, p>0.05, Fig. 4.8d). Overall, female rats had fewer EHE's compared to male rats (2-Factor ANOVA, p<0.001, Fig. 4.8d).

As an indirect measurement of the overall impact of EHE's on MAP, we also calculated the area under the curve (AUC, $\mathrm{mmHg} / \mathrm{s}$ ) of the spikes. To do this, we took the integral of each EHE during REM sleep. For each animal, we then took a sum (SumAUC) of these EHE integrals. In 2-3 month-old rats, the SumAUC was not different between TPH2-/- rats and WT controls, irrespective of sex (2-Factor ANOVA, p>0.05, Fig. 4.8e). By 5-8 months of age, male TPH2-/- had an increased SumAUC compared to WT controls (2-Factor ANOVA, p<0.001, Fig. 4.8f). Further, female TPH2-/- rats had a reduced SumAUC compared to male TPH2-/- rats (2-Factor ANOVA, genotype*sex interaction $\mathrm{p}<0.004)$. By 12-13 months of age, the SumAUC was not different between $T P H 2-/$ - rats and WT controls, irrespective of sex (2-Factor ANOVA, p>0.05, Fig. 4.8g). Female rats had a smaller SumAUC than male rats (2-Factor ANOVA, sex, p<0.001, Fig. 4.8g).

In summary, at 5-8 months of age, central 5-HT deficiency leads to an increased number of EHE's during REM sleep, irrespective of gender. Further, the SumAUC of these events was also elevated during REM sleep, but only in males. By 12-13 months of age, while both genotypes still exhibited EHE's during REM, there was no effect of genotype on the number of EHE's nor the SumAUC of these events.

\section{Central 5-HT does not contribute to the resting control of breathing}

Neonatal $\mathrm{TPH} 2$-/ rats hypoventilate in all states, and have increased numbers of apneas in active sleep [214]. Therefore, we monitored breathing during sleep to determine whether this phenotype persisted into adulthood. In 2-3, 5-8, and 12-13 month old male 
and female rats, breathing at rest was largely unaffected by chronic 5-HT depletion (Table 4.5 and 4.6).

In 2-3 month old rats, there was no significant effect of a loss of central 5-HT on $\mathrm{V}_{\mathrm{E}} / \mathrm{VO}_{2}$ in any state. However, there were significant effects of genotype on the pattern of breathing across vigilance states. For example, the frequency of breathing (fB) in male and female WT animals increased from NREM to REM sleep, while in TPH2-/- rats, fB decreased from NREM to REM (2WRMA, genotype*vigilance-state interaction $\mathrm{p}=0.013$ ).

Similar to 2-3 month old rats, there was no effect of central 5-HT deficiency on $\mathrm{V}_{\mathrm{E}} / \mathrm{VO}_{2}$ across states in 5-8 month old rats. Similar to younger rats, the fB of 5-8 month old male and female WT rats increased from NREM to REM, an effect of state that did not occur in TPH2-/- littermates (2WRMA, genotype*vigilance-state $\mathrm{p}<0.001$ ).

Similar to younger animals, $\mathrm{V}_{\mathrm{E}} / \mathrm{VO}_{2}$ was not influenced by a loss of central 5-HT in 12-13 month old rats. Interestingly, in contrast to younger TPH2-/- rats, fB of 12-13 month old $\mathrm{TPH} 2-/$ - rats increased from NREM to REM sleep; in females, this increase was more than double the increase observed in WT rats (2WRMA, genotype*vigilance-state $\mathrm{p}=0.007)$. We also calculated the coefficient of variability of the respiratory period $(\mathrm{CVP} \%)$ as a measure of respiratory stability. Similar to reports suggesting respiratory instability during REM sleep [92], CVP\% was greatest in REM at all three ages, but neither genotype nor sex had any influence (2WRMA, vigilance-state $\mathrm{p}<0.001)$.

In summary, central 5-HT does not contribute to overall resting ventilation in adult rats. However, the pattern of breathing (i.e. fB) across vigilance-states was affected by genotype. For instance, in 2-3 month old WT rats, fB increased from NREM to REM, but decreased from NREM to REM in $T P H 2-/-$ rats. 


\section{5 - DISCUSSION}

In this study, we addressed the hypotheses that central 5-HT prevents MAP from falling, mainly during QW and NREM sleep, when 5-HT neurons are active. However, we found that by $\sim 6-$ months of age, rats lacking central 5-HT had an elevated MAP compared to WT rats, across all vigilance states, but was most obvious during REM sleep. Therefore, we sought to determine the mechanisms associated with this phenotype. We hypothesized that central 5-HT reduces sympathetic drive [299] and/or angiotensin II (Ang II) activity [29], as neurogenic hypertension is associated with these phenotypes. However, we found that neither sympathetic drive nor circulating Ang II contribute to the elevated MAP seen in rodents lacking central 5-HT.

\section{Effects of a loss of central 5-HT on sleep in adult rodents}

Regardless of age and sex, TPH2-/- rats exhibited prolonged REM episodes compared to WT controls, similar to what we saw during the neonatal period [214]. This suggests that central 5-HT is involved in promoting wakefulness and inhibiting REM sleep generation, consistent with previous reports $[5,132,133,303]$. The mechanism is likely through the laterodorsal (LdT) and pedunculopontine (PPT) tegmentii cholinergic neurons, which send projections to and activate glutamatergic neurons of the pontine reticular formation to initiate and maintain REM sleep [5]. The LdT/PPT also receives 5-HT input from the dorsal raphe [304]. When applied to the LdT, 5-HT reduces REM sleep, presumably through activation of the inhibitory $5-\mathrm{HT}_{1 \mathrm{~A}}$ receptor $[132,305]$. Taken together, with the results of these previous studies, our findings suggest that prolonged 
REM episodes in TPH2-/- adults is through disinhibition of LdT and/or PPT resulting from a loss of $5-\mathrm{HT}_{1 \mathrm{~A}}$ mediated signaling.

Despite their prolonged REM episodes, overall time spent in REM is the same in male and female $T P H 2-/-$ and sex-matched WT rats. This is because $T P H 2-/-$ rats have fewer episodes of REM compared to WT controls. The LdT/PPT express excitatory 5-HT2 receptors [306] and activation of 5- $\mathrm{HT}_{2 \mathrm{~A}}$ receptors increases the number of REM episodes in adult rodents [307]. Overall, 5-HT deficiency may reduce the ability of cholinergic neurons to turn on (through a loss of excitatory $5-\mathrm{HT}_{2 \mathrm{~A}}$ receptor signaling) and turn off when activated (through a loss of inhibitory $5-\mathrm{HT}_{1 \mathrm{~A}}$ signaling). Thus, the consequences for TPH2-/- adult rats are fewer, but longer, REM episodes, similar to what we have seen during the neonatal period [214] .

\section{Effects of a loss of central 5-HT on resting MAP and HR during sleep in adult rodents}

The increased ABP in male and female 5-8 and male 12-13 month TPH2-/- rats are consistent with previous studies utilizing adult rats treated with $p$-chlorophenylalanine ( $p$ CPA), which had a significant rise in baseline MAP [210]. However, since $p$-CPA induces a systemic loss of 5-HT, whether this effect was primarily due to a loss of peripheral or central 5-HT was not clear. Our current data contradicts previous reports showing reduced, not increased, MAP in TPH2 knockout mice, mainly during the early evening [224]. This discrepancy may be attributed to species related differences in the control of MAP. However, we have previously found that neonatal $\mathrm{TPH} 2-/$ - rats had a reduced MAP during quiet sleep (similar to NREM sleep in adults) [298]. It may be that the reduced MAP of rats deficient in central 5-HT induces a mechanism that initially compensates for the loss 
of MAP, but which, when chronically active, eventually leads to an overshoot in MAP, leading to hypertension in adulthood. Many reports suggest that fetal programming during critical stages of development may lead to elevated MAP during adulthood [308].

The elevated MAP in 5-8 month old $T P H 2-/$ rats was mediated by both an elevated sBP and dBP while by 12-13 months, it was mediated via increased dBP only, irrespective of vigilance-state. As has been demonstrated by other studies using adult animals, our results suggest that central 5-HT signaling reduces vascular resistance, with little influence on ventricular contractility [288]. Given these results, we calculated the power spectral analysis of the low frequency (LF\%) component of sBP, an indirect measure of sympathetic nerve activity [300, 301]. At least in 6 month old rats, LF\% was elevated in male $\mathrm{TPH} 2$-/ rats compared to WT controls, further suggesting that sympathetic activity to the vasculature may be elevated. Therefore, we induced a full ganglionic block (with atropine and hexamethonium), as a more direct measure of sympathetic vascular tone [310]. Despite an elevated LF\% in male TPH2-/- rats, the response to ganglionic blockade was not influenced by genotype, suggesting that 5-HT does not contribute to sympathetic vascular tone. In 6-month old female rats, neither LF\% nor the response to ganglionic blockade were influenced by a loss of central 5-HT. Some reports in the spontaneously hypertensive rat suggest that LF\% of sBP does not reflect splanchnic nerve activity [311], which may at least partially explain why we saw genotype differences in male rats for LF\% but not in the response to ganglionic blockade. Hence, we think the responses to ganglionic blockade are more reflective of sympathetic nerve activity in our model. However, given the equivocacy of indirect measurements, direct sympathetic nerve recordings will be needed to assess whether central 5-HT influences sympathetic discharge. 
We also measured the very low (vLF\%) component of sBP, which is an indirect measure of sympathetic outflow related to hormonal activity or thermoregulation $[300$, 301]. Adult $T P H 2-/-$ rats appear to thermoregulate normally compared to WT controls [155]; therefore it is conceivable that the elevated vLF\% in male 6-month old TPH2-/- may reflect changes in sympathetic outflow related to hormonal activity. We hypothesized that altered neurohumoral control of MAP, through elevated angiotensin II (Ang II), might play a role in the development of hypertension in these $\mathrm{TPH}_{2}-\mathrm{I}_{-}$rats. Ang II is a potent vasoconstrictor [29, 299] and evidence suggests that Ang II can potentiate sympathetic activity via stimulating sympathetic ganglionic activity, and/or increasing epinephrine and norepinephrine release from the adrenal medulla [25]. Reports also suggest that the reninangiotensin system may interact with central 5-HT. Indeed, in adult rats, plasma renin activity is elevated following a stressor in rats where the median raphe is lesioned, compared to control rats [166], suggesting that a loss of serotonergic tone increases renin activity, and possibly the renin-angiotensin system.

Our original experiments sought to address the hypothesis that Ang II would be elevated in the absence of central 5-HT and its actions would predominately be mediated through the CNS. This was, in part, stemming from our experiments showing sympathetic drive did not appear to mediate the MAP phenotype in TPH2-/- rats (Fig. 4.3b, left). In our original set of experiments (Fig. 4.6), we performed a ganglionic blockade plus an AT1x and V1Ax to assess the contributions of Ang II and AVP to MAP in the absence of autonomics. However, in this small set of rats, we found that MAP was not elevated at rest in $T P H 2$-/- rats compared to WT controls. Further, the response to ganglionic blockade was not different between genotypes, as we saw in previous experiments (Fig. 4.4b). Therefore, 
we are unable to accurately interpret this data. It may be that in such a small sample size, our phenotype is lost. However, as seen in the larger dataset, it is clear that a loss of central 5-HT elevates MAP beginning at 5-8 months of age. In a separate set of rats, we decided to inject losartan in the absence of complete autonomic blockade, allowing us to directly assess whether elevated Ang II signaling (whether central or peripheral) contributes to the elevated MAP of TPH2-/- rats. Following AT1 blockade, the MAP phenotype still persisted in $\mathrm{TPH} 2-/-$ rats. These results suggest that Ang II does not contribute to resting MAP in our 5-HT deficient model, irrespective of its site of action.

Essential hypertensive patients exhibit an exaggerated rise in blood pressure in the morning, a phenomenon referred to as the morning ABP surge [312]. This surge is known to increase the risk of cardiovascular events [313]. Interestingly, ABP surges are prevalent during REM sleep [314]; interestingly, this phenotype is present in spontaneously hypertensive rats during REM sleep compared to controls [247]. We observed "extreme hypertensive events" ("EHE") or BP surges (periods of BP increases greater than $20 \mathrm{mmHg}$ over baseline) in REM sleep and sought to assess whether 5-HT might play a role in this phenomena. We found that only at 5-8 months of age, male and female TPH2-/- had a higher prevalence of these EHE's compared to WT rats. Further, when assessing the impact of these EHE's, we calculated the sum of the area under the curve (SumAUC). Only 5-8 month old male rats had an increased SumAUC, suggesting that the impact of EHE's to the elevated MAP during REM was greater in males than in females. Although there was no significant interaction between sex and central 5-HT deficiency on MAP regulation at this age, the lack of central 5-HT appeared to be especially deleterious for males. Ultimately, there was not a correlation between EHE's and MAP. Further, we were unable 
to determine the contribution of autonomics to EHE's; following ganglionic blockade, our rats did not return to REM sleep.

The elevated EHE's and SumAUC may be related to REM episode length, as both male and female $\mathrm{TPH} 2-/-$ rats had increased REM episode durations compared to WT controls, irrespective of age. However, given that at 12-13 months of age, male TPH2-/rats have an increased MAP during REM without any increase in the frequency of EHE's, it is unlikely that EHE's contribute to their increased MAP at this age. If anything, HR increased during EHE's, suggesting that an altered baroreflex sensitivity (BRS) is likely not contributing to these events during REM sleep. However, the relative contribution of BRS to MAP and EHE's during REM sleep remains to be elucidated in our model. Another contributing factor to EHE's may be midbrain dopaminergic neurons, which originate in the ventral tegmental area (VTA). Blockade of dopaminergic neurons within the VTA with 6-hydroxydopamine (6-OHDA) suppresses ABP surges during REM sleep [315], suggesting these neurons participate in the presence of EHE's. 5-HT may exert control over VTA dopaminergic neurons. Evidence suggests that blockade of 5- $\mathrm{HT}_{2}$ receptors within the VTA induces a dose-dependent increase in dopaminergic neurotransmission [316], suggesting that 5-HT exerts an inhibitory control of midbrain dopaminergic activity. It may be that, in the absence of 5-HT, dopaminergic signaling contributes to the increase in MAP surges during REM sleep. However, this remains to be tested, especially since by 12-13 months of age, the prevalence of EHE's is not affected by a loss of central 5-HT.

At this point, we can only speculate as to the mechanism mediating elevated MAP in male $T P H 2-/-$ rats. One potential mechanism is that baroreflex sensitivity (BRS) may be reduced in $\mathrm{TPH} 2-/-$ rats. Indeed, both the spontaneously hypertensive rat [317] and patients 
with EH [318] exhibit reduced BRS. Furthermore, evidence suggests that central 5-HT plays a role in BRS; 5-HT depletion with $p$-CPA not only increases MAP, but also reduces BRS [210]. While we still need to perform additional tests assessing BRS in our model, TPH2-/- tended to have a reduced BRS compared to WT controls (Supplemental Figure 4.9, 2WRMA, p=0.04). However, evidence from sinoaortic denervation (SAD) studies shows that SAD results in only a transient increase in MAP and sympathetic nerve activity in animal models, where both MAP and SNA eventually return to near normal levels, which argues against a role for altered BRS in the long term control of MAP [319]. Therefore, at least in older $\mathrm{TPH}$-/- rats, the baroreflex may have reset and other mechanisms are contributing to the elevating MAP seen in central 5-HT deficiency.

The elevated MAP phenotype in TPH2-/- rats may be mediated through the somatodendritic autoinhibition of 5-HT neurons with 5-HT 1 A autoreceptors [170]. Activation of $5-\mathrm{HT}_{1 \mathrm{~A}}$ autoreceptors potentially reduces the release of not only 5-HT, but also coexpressed neuromodulators. The lack of 5-HT in TPH2-/- rats reduces the possibility of 5$\mathrm{HT}_{1 \mathrm{~A}}$ mediated autoinhibition of 5-HT neurons. Therefore, it may be that $\mathrm{TPH} 2-/-$ rats have an elevated release (compared to WT counterparts) of these co-expressed neuromodulators like substance $\mathrm{P}$ and TRH $[150,151]$, which have well known roles in cardiovascular homeostasis [296, 297].

\section{Female rats are generally protected from the deleterious effects of 5-HT deficiency}

At 5-8 months of age, female TPH2-/- rats, like their male counterparts, have an increased MAP compared to WT controls, albeit to a lesser degree than male $T P H 2-/-$ rats. This phenotype was absent at 2-3 and 12-13 months of age (compare male vs. female Fig. 
4.2b). It appears that female $T P H 2-/$ - rats are generally protected from the elevated MAP seen in male $T P H 2-/$ - rats; although further testing in 5-8 month old female rats is warranted, to tease out the mechanisms associated with this age-dependent increase in MAP in 5-HT deficiency in female rats. It is well established that sex influences the control of MAP with aging. Indeed, pre-menopausal women ( $<50$ years old) have a lower incidence of hypertension than age-matched men, and have an increased incidence after age 55 [291]. Estrogen receptors (ER) are expressed within several brain regions key to the regulation of sympathetic nerve activity (SNA), HR, and MAP, including the paraventricular nucleus of the hypothalamus (PVN) and rostral ventrolateral medulla (RVLM) [320]. Within the RVLM, activation of ER $\alpha$ receptors with estrogen decreases SNA [321]. 5-HT receptors can be found in the same locations as estrogen receptors [4, $131,159,322,323]$ and ovarian steroids have been shown to modulate several genes of the 5-HT system, including tryptophan hydroxylase, the vesicular monoamine and 5-HT transporters, and several of the 5-HT receptors [324, 325]. Ovarian hormones increase tryptophan hydroxylase expression in rhesus monkeys [326]. This could increase 5-HT binding to inhibitory $5-\mathrm{HT}_{1 \mathrm{~A}}$ receptors located within the RVLM, promoting a reduction in MAP [163] in female WT controls that would be absent in TPH2-/- rats. Therefore, while it is possible that ovarian steroids are mediating their effects directly on 5-HT neurons [326], it is equally possible that estrogen is compensating for the loss of central 5-HT mediated inhibition of sympathoexcitatory regions (like the RVLM) to normalize the MAP through its effects on ER's [321].

Effects of a loss of central 5-HT on resting ventilation during sleep in adult rodents 
Several models of 5-HT deficiency produce respiratory abnormalities. For instance, infant mice deficient in Lmx1b $\left(\operatorname{Lmxl} b^{f f f p}\right)$, which selectively lack 5-HT neurons, have frequent and severe apneas, lasting up to 55s in length [213]. More recent data in neonatal TPH2-/- rats suggest that a specific loss of 5-HT also plays a role in respiratory function. Like $L m x l b^{f f f / p}$ mice, as neonates these rats hypoventilate at rest and have frequent and severe apneas [155], a phenotype particularly evident in active sleep [214]. Further, this phenotype is normalized by adulthood. Similar to the MAP phenotype described herein, these previous findings suggest that in the absence of central 5-HT, there are compensatory mechanisms in neural circuits governing ventilatory control [155]. Our data largely corroborates these results, showing that chronic CNS 5-HT depletion does not affect ventilation during sleep in adulthood. One potential mechanism includes the ontogeny of adenosinergic receptors, in particular the $\mathrm{A}_{2 \mathrm{~A}}$ receptor. These receptors are high during the second and third postnatal week and decline between 21 days and adulthood [327], suggesting that the effect of adenosine becomes less potent as a respiratory depressant in adult rats [328] - wherein 5-HT during the neonatal period may compensate for the inhibitory actions of adenosine to maintain normal respiration.

\section{Methodological Considerations}

At the end of our experiments, we determined the effectiveness of losartan on blocking AT1 receptors and hexamethonium on blocking sympathetic ganglia by injecting Ang II (i.v. $25 \mathrm{ng} / \mathrm{kg}$ ) and phenylephrine (PE, i.v. $10 \mu \mathrm{g} / \mathrm{kg}$ ), respectively. In rats treated with Losartan + ganglionic blockade, Ang II did not produce a significant change in MAP or HR in our model (Supplemental Figure 4.10), suggesting that blockade of these receptors 
was complete. Following ganglionic blockade (in the presence of AT1x), there was a small baroreflex mediated heart rate response following injection of phenylephrine (PE, Supplemental Figure 4.11), suggesting that, at the time of PE injection, the block was not complete. Both WT and TPH2-/- rats exhibited a similar fall in HR to the MAP following ganglionic blockade, suggesting that the block obtained in both groups was comparable. Furthermore, previous reports have suggested that: 1) hexamethonium at the dose we used for assessing sympathetic tone adequately blocked sympathetic output and 2) hexamethonium produced similar falls in pressure as other ganglionic blockers, like chlorisondamine, which show a more effective blockade of the baroreflex mediated change in HR [310, 329]. Taken together, these findings suggest that hexamethonium provides an accurate index of sympathetic drive to the vasculature. However, in light of the presence of a baroreflex mediated change in HR, it may be worthwhile to directly measure sympathetic nerve activity to obtain a more direct assessment of 5-HT's role on sympathetic nerve activity. The influence of central 5-HT on circulating norepinephrine or epinephrine concentrations could also be measured.

The elevated MAP in TPH2-/- rats does not appear to be mediated by elevated sympathetic drive or Ang II; therefore, we assessed whether elevated AVP may be mediating the phenotype (Supplemental Figure 4.12). We only utilized 2 male TPH2-/- and WT controls and, at this point, the responses to Manning Compound (and thus, the contribution of AVP to resting MAP) are inconclusive (Supplemental Figure 4.12b). Further, we noticed that following blockade of V1A receptors with Manning Compound, these rats still exhibited a small but significant response to AVP (Supplemental Figure 4.13); suggesting blockade of AVP was not complete. We note, however, that the doses we 
used in our experiments were consistent with previous reports where blockade of V1A receptors was complete [330]. Considering the low “n”, additional animals are needed to verify the contribution of AVP to resting MAP in our model.

An unanticipated finding was that, at 5-8 months of age, female TPH2-/- rats, like their male counterparts, also have increased MAP compared to WT, albeit to a lesser degree than male TPH2-/- rats. This phenotype was absent at 2-3 and 12-13 months of age. There are reports suggesting that brain 5-HT levels are influenced by the phases of the estrous cycle [325]. Although we did not determine estrous stage in our animals, the phases of the estrous cycle could contribute to the higher ABP of 5-8 month old TPH2-/- rats as well as the higher variability of ABP in our females compared to males (some of the TPH2-/- rats may be in estrous and therefore relatively "protected" from the influence of 5-HT deficiency). In addition to future studies to determine whether the elevated MAP is restricted to any portion of the estrous cycle, future studies could assess whether ovariectomy exaggerates the phenotype in female $\mathrm{TPH} 2$-/- rats or if gonadectomy reverses the phenotype in male $T P H 2-/$ rats.

\section{6 - PERSPECTIVES AND SIGNIFICANCE}

Ultimately, our goal is to better understand the role of central 5-HT in MAP regulation during sleep. Our findings suggest that a loss of central 5-HT may be a contributing factor to neurogenic hypertension [233]. This could be due to a loss of inhibitory $5-\mathrm{HT}_{1 \mathrm{~A}}$ signaling in key presympathetic regions in the brain, such as the RVLM [199, 309]. Given that the elevated MAP of TPH2-/- rats appears to not be related to increased sympathetic drive (at least indicated by our indirect measurements) or Ang II 
signaling, it may be that other neurohumoral agents, like AVP [331], contribute to the elevated $\mathrm{ABP}$ in $\mathrm{TPH} 2-/-$ rats. Alternatively, considering BRS contributes to MAP regulation in both the spontaneously hypertensive rat [317] and patients with EH [318], BRS may be reduced in TPH2-/- rats (Supplemental Fig. 4.9).

Females appear to be generally protected from the phenotype, suggesting that there may be an interaction between central 5-HT and female sex hormones. 5-HT and estrogen receptors can be found in the same locations that modulate cardiovascular control, including the PVN and RVLM [4, 131, 159, 322, 323]. Estrogen may compensate for the loss of central 5-HT mediated inhibition of sympathoexcitatory regions (like the RVLM) to normalize the MAP through its effects on ER's [321].

\section{7 - ACKNOWLEDGEMENTS}

We thank Jane Chen for technical assistance and animal husbandry, Dr. Matthew Hodges (Medical College of Wisconsin) for providing the animal model, and Drs. Eileen Hasser and Cheryl Heesch for helpful discussions throughout this study. 


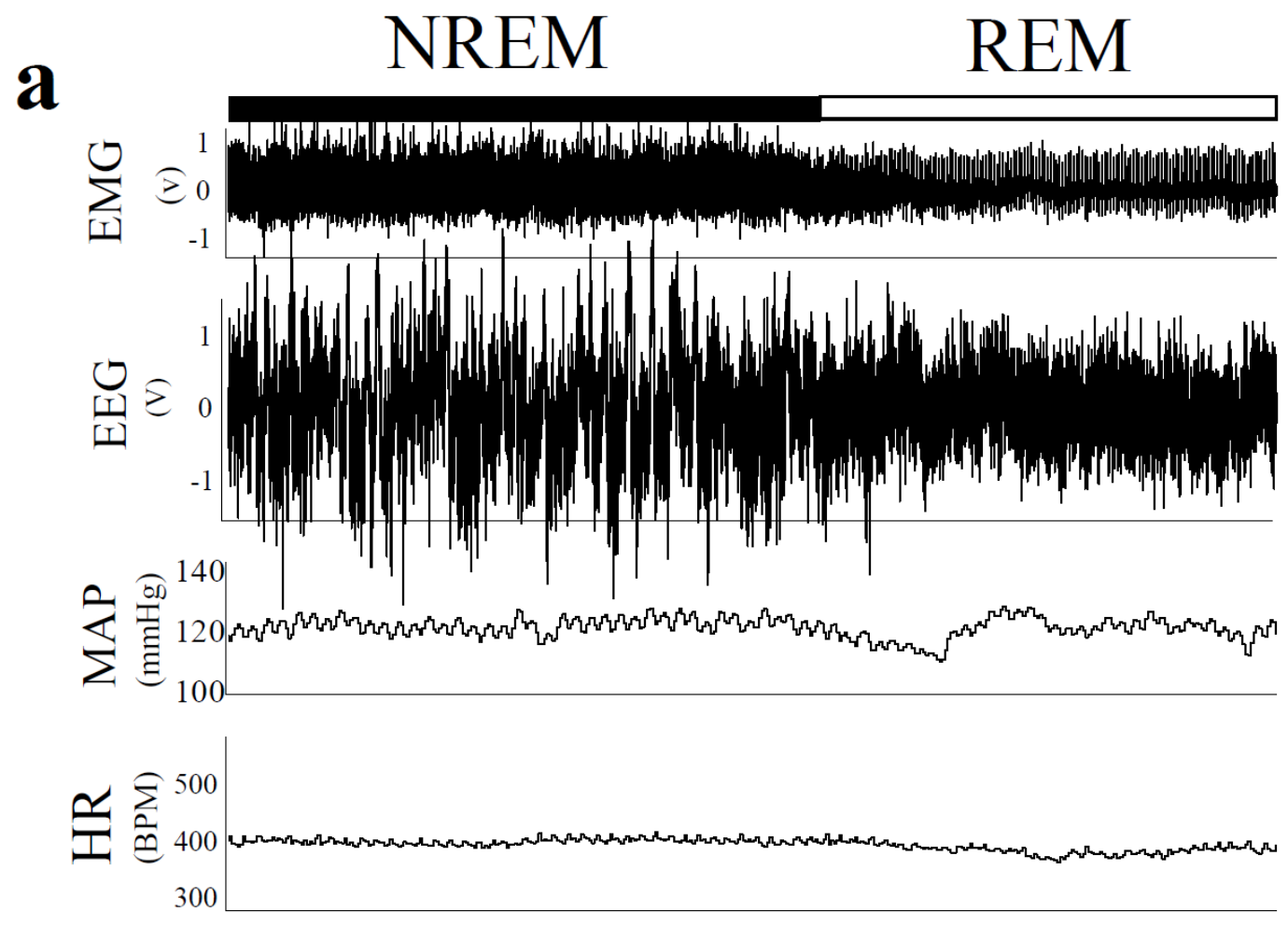

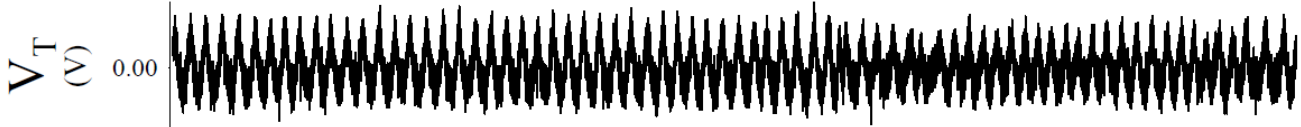

b

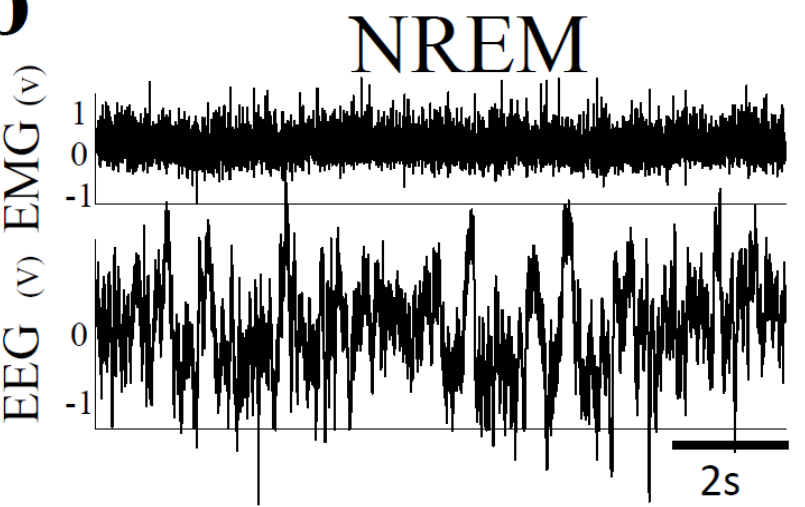

$10 \mathrm{~s}$

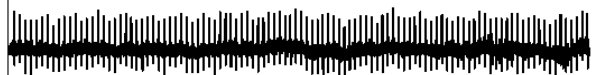

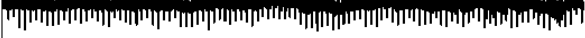

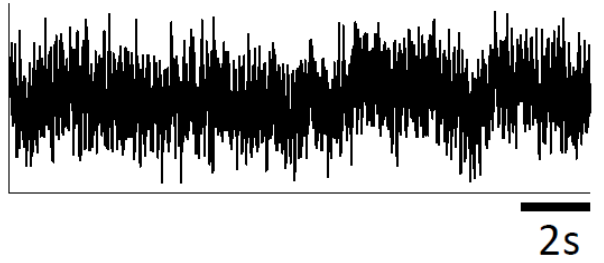

Figure 4.1: a) Electromyograph (EMG, v), Electroencephalograph (EEG, v), mean arterial pressure (MAP, $\mathrm{mmHg}$ ), heart rate ( $\mathrm{HR}$, beats/min), and tidal volume $\left(\mathrm{V}_{\mathrm{T}}\right)$ traces obtained during periods of NREM (black bar) and REM (white bar). Frequency of EEG and amplitude of EMG allow us to compare different sleep states for the measurement of cardiorespiratory variables. During acquisition, NREM was defined as a continuous, synchronized, low frequency $(0.5-4 \mathrm{~Hz})$ and high voltage EEG with an amplitude two to three times greater than observed in QW. REM sleep was characterized by desynchronized, low-voltage, high frequency EEG with the lowest observed EMG amplitude. Scale bar: 10s. b) Expanded view of NREM (left) and REM (right). Scale bar: $2 \mathrm{~s}$ 


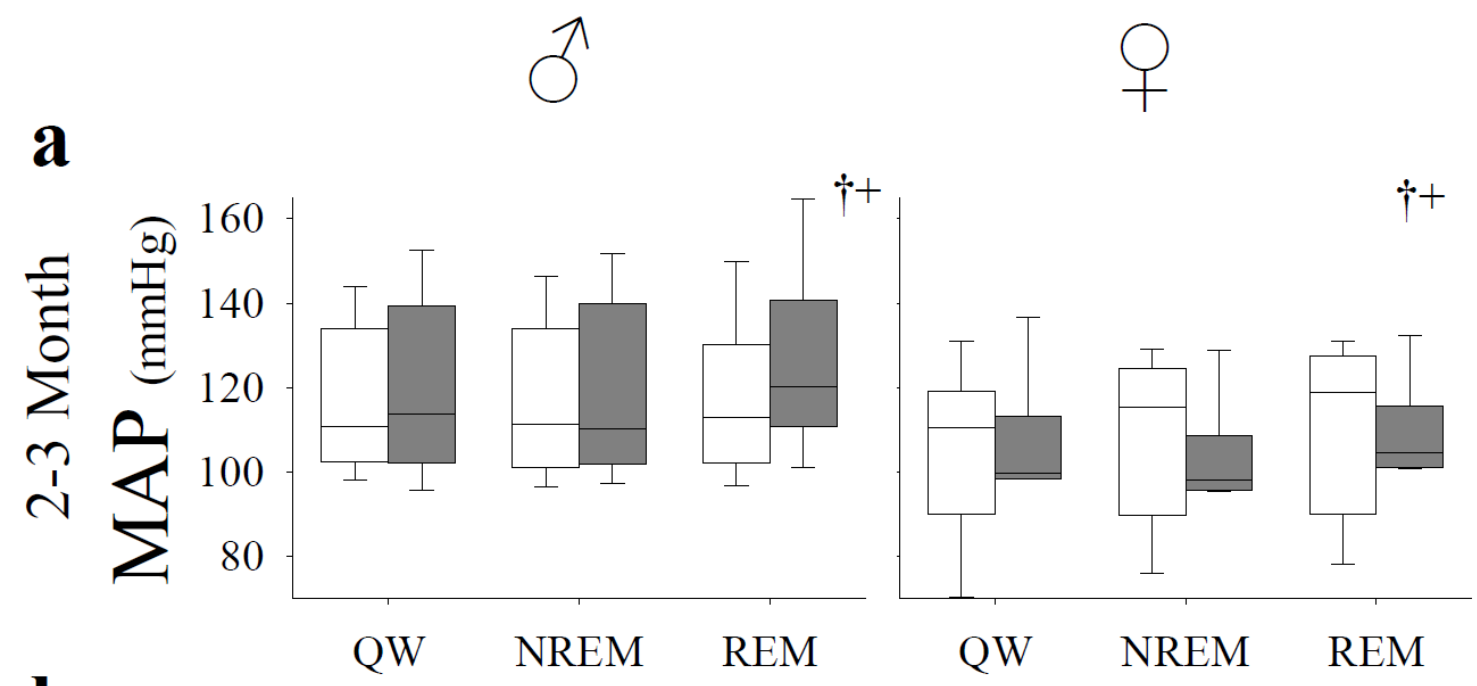

b
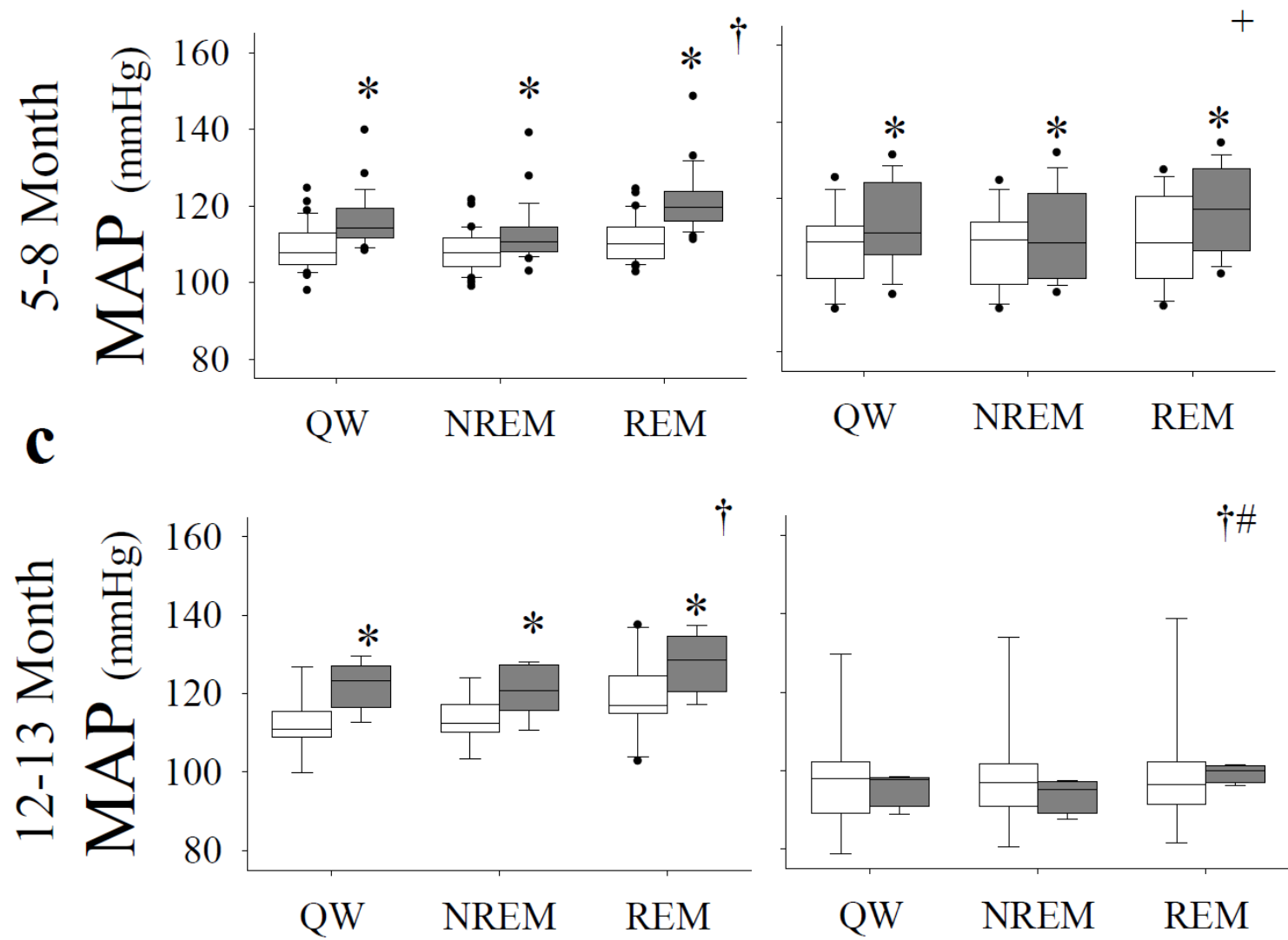

Figure 4.2: Central 5-HT deficiency leads to elevated blood pressure in 5-8 and 12-13 month-old male rats. Shown is mean arterial pressure (MAP, $\mathrm{mmHg}$ ) in male (left) and female (right) $\mathrm{TPH} 2-/$ (dark gray) and WT (open) rats at a) 2-3 month (TPH2-/- male $\mathrm{n}=8$, female $\mathrm{n}=6$ and WT male $\mathrm{n}=9$; female $\mathrm{n}=9$ ), b) 5-8 months (TPH2-/- male $\mathrm{n}=28$, female $\mathrm{n}=15$; WT male $\mathrm{n}=31$, female $\mathrm{n}=12$ ), and c) $12-13$ months $($ TPH2-/male $n=6$, female $n=4$; WT male $n=9$, female $n=7$ ) of age. *: genotype $p<0.05$; $\uparrow$ : vigilance-state $p<0.05$; \#: Sex $\mathrm{p}<0.005 ;+$ : genotype*vigilance-state interaction $\mathrm{p}<0.05$; $\$$ : genotype*vigilance-state*sex Interaction $\mathrm{p}<0.05$. Data are shown as box plots. Shown are the median (line through the box) as well as the $25^{\text {th }}$ and $75^{\text {th }}$ percentiles (bottom and top edges of box) and the $10^{\text {th }}$ and $90^{\text {th }}$ percentiles (error bars) with outliers shown as individual points. 


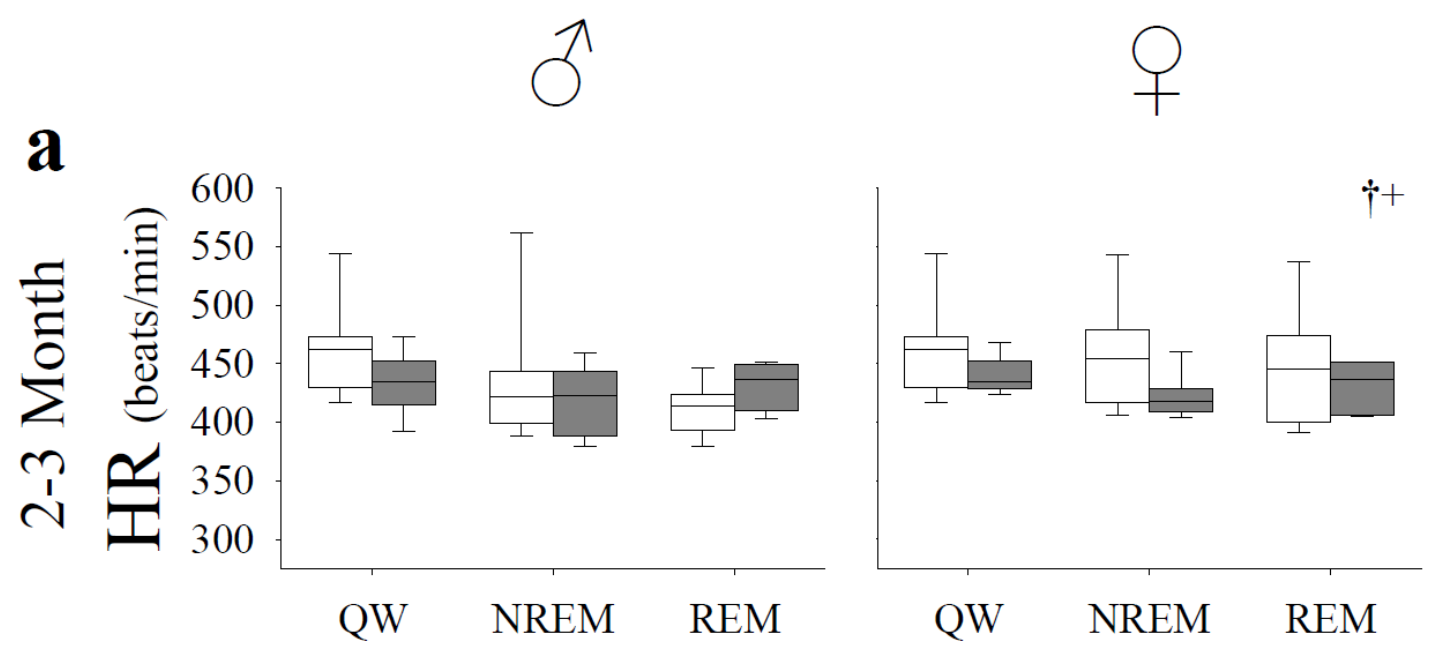

b
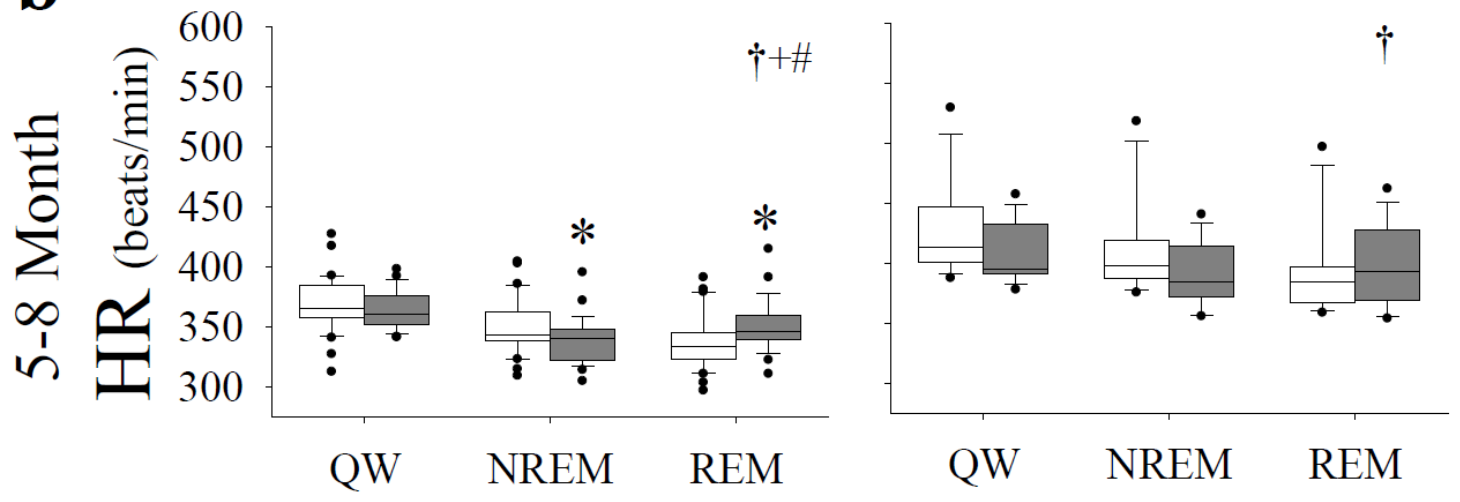

C
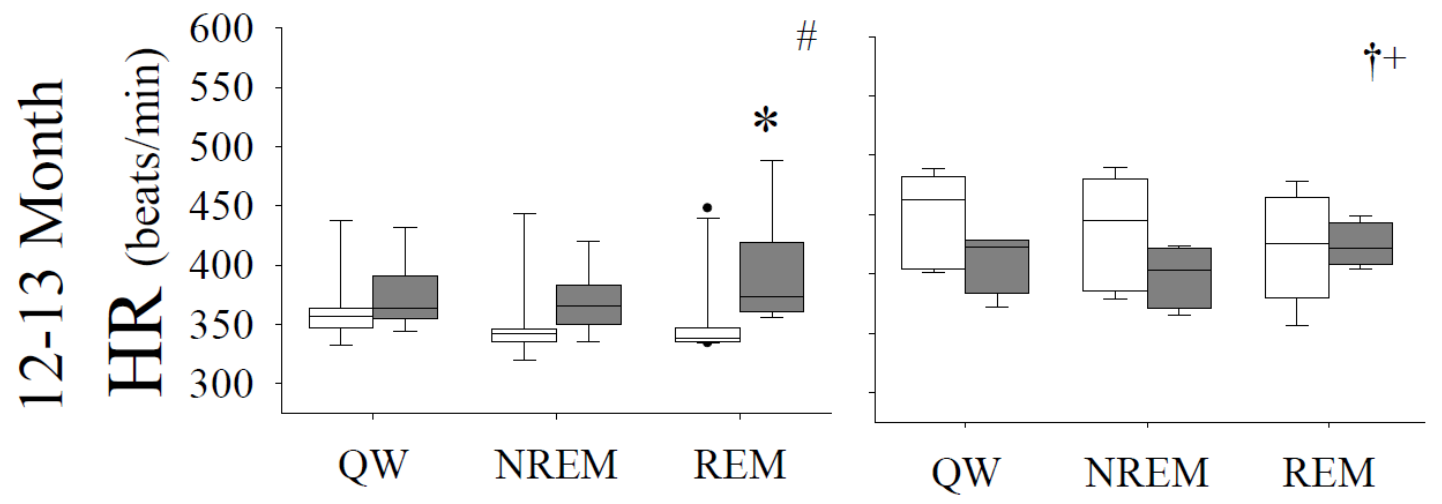

Figure 4.3: Central 5-HT deficiency does not affect HR during sleep in adult rats. Shown is heart rate (HR, beats/min) in male (left) and female (right) TPH2-/- (dark gray) and WT (open) rats at a) 2-3 month (TPH2-/- male $\mathrm{n}=8$, female $\mathrm{n}=6$ and WT male $\mathrm{n}=9$; female $\mathrm{n}=9$ ), b) 5-8 months (TPH2-/- male $\mathrm{n}=28$, female $\mathrm{n}=15$; WT male $\mathrm{n}=31$, female $\mathrm{n}=12$ ), and c) $12-13$ months (TPH2-/- male $\mathrm{n}=6$, female $\mathrm{n}=4$; WT male $\mathrm{n}=9$, female $\mathrm{n}=7$ ) of age. *: genotype $\mathrm{p}<0.05$; $\uparrow$ : vigilance-state $\mathrm{p}<0.05$; \#: sex $\mathrm{p}<0.005 ;+$ : genotype*vigilancestate interaction $\mathrm{p}<0.05 ;+$ : genotype*vigilance-state*sex interaction $\mathrm{p}<0.05$. Data are shown as box plots. Shown are the median (line through the box) as well as the $25^{\text {th }}$ and $75^{\text {th }}$ percentiles (bottom and top edges of box) and the $10^{\text {th }}$ and $90^{\text {th }}$ percentiles (error bars) with outliers shown as individual points. 


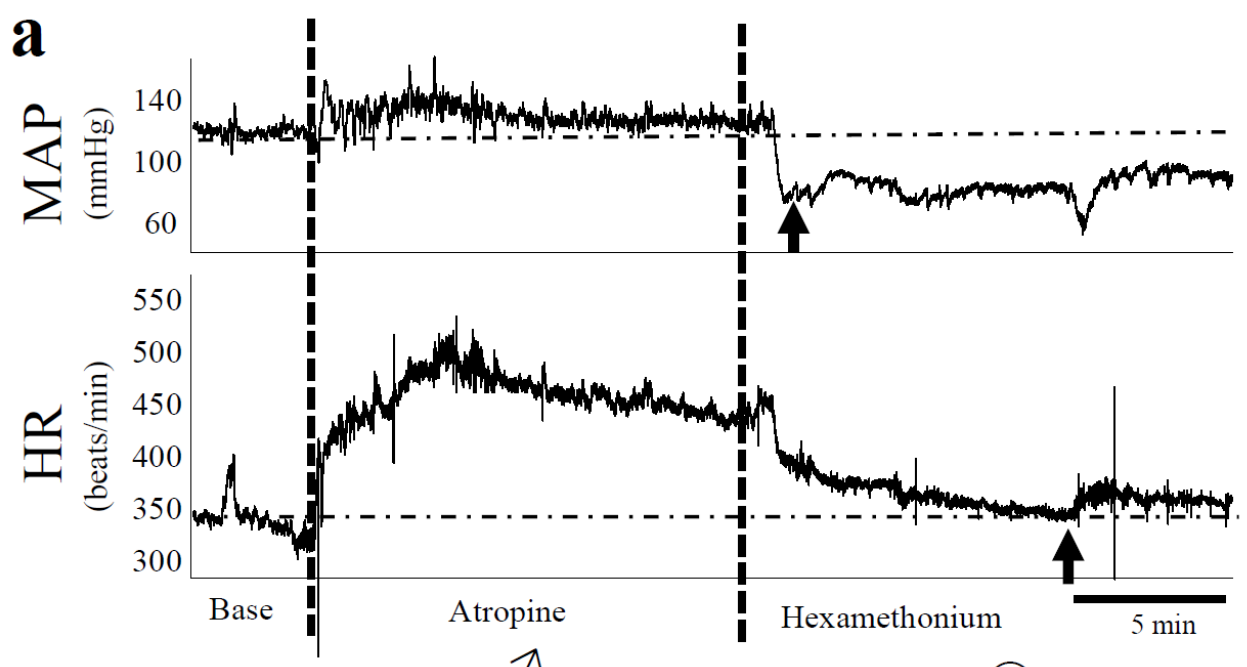

b

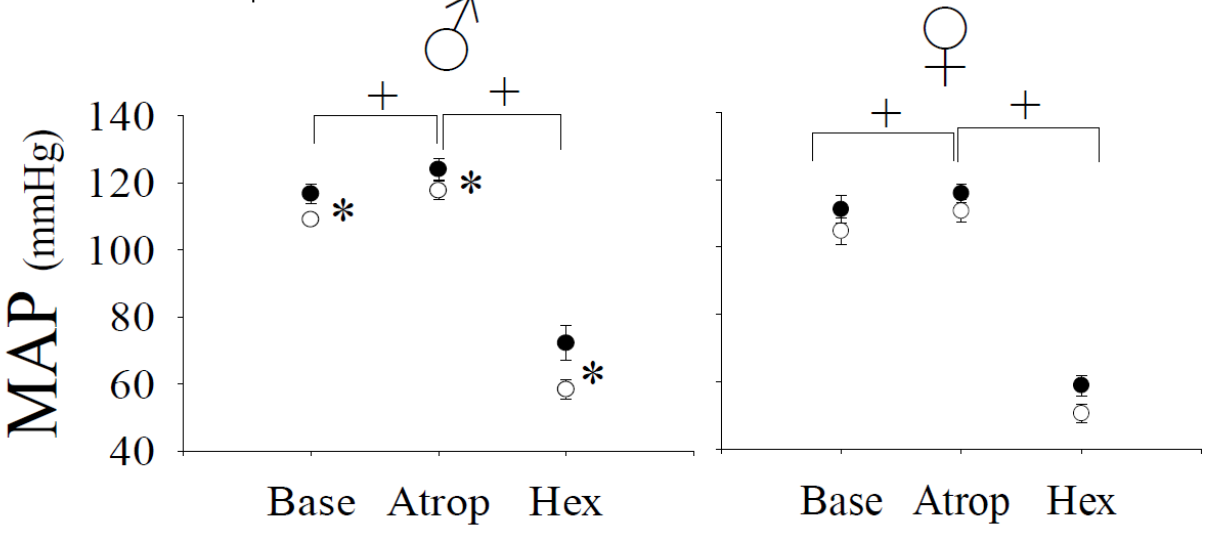

C

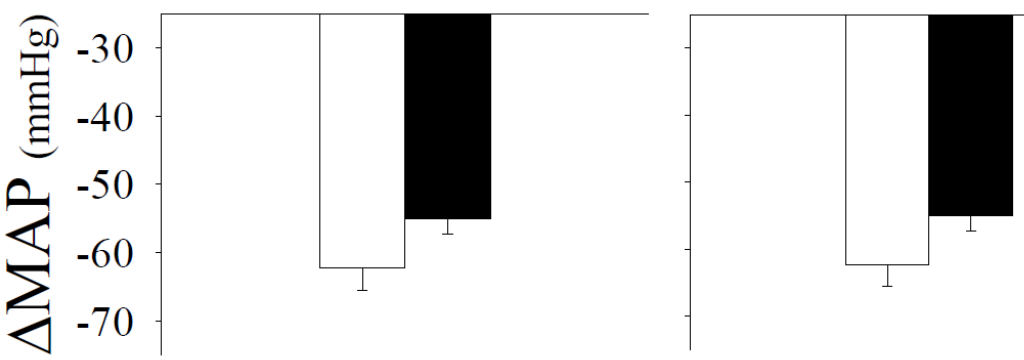

Figure 4.4: Sympathetic tone to the vasculature does not contribute to the elevated ABP in central 5HT deficiency. a) Shown are typical responses in QW of mean arterial pressure (MAP, mmHg, top) and heart rate (HR, beats $/ \mathrm{min}$, bottom) of a WT rat to atropine methyl nitrate (Atrop, i.v. $1 \mathrm{mg} / \mathrm{kg}$, Sigma Chemical) and hexamethonium (Hex, i.v. 30mg/kg, Sigma Chemical). Black arrows indicate maximal response in MAP and HR following Hex. b) Average responses shown of male (left) and female (right) TPH2-/- (closed circles, male $\mathrm{n}=11$, female $\mathrm{n}=9$ ) and WT controls (open circles, male $\mathrm{n}=11$, female $\mathrm{n}=8$ ). $\mathbf{c}$ ) $\triangle \mathrm{MAP}$ in response to Hex of male (left) and female (right) in WT (open bars) and TPH2-/- (closed bars) during QW. *: genotype $\mathrm{p}<0.05$; +: effect of treatment $\mathrm{p}<0.05$. Data are mean values \pm standard error of the mean. 


\section{GB}

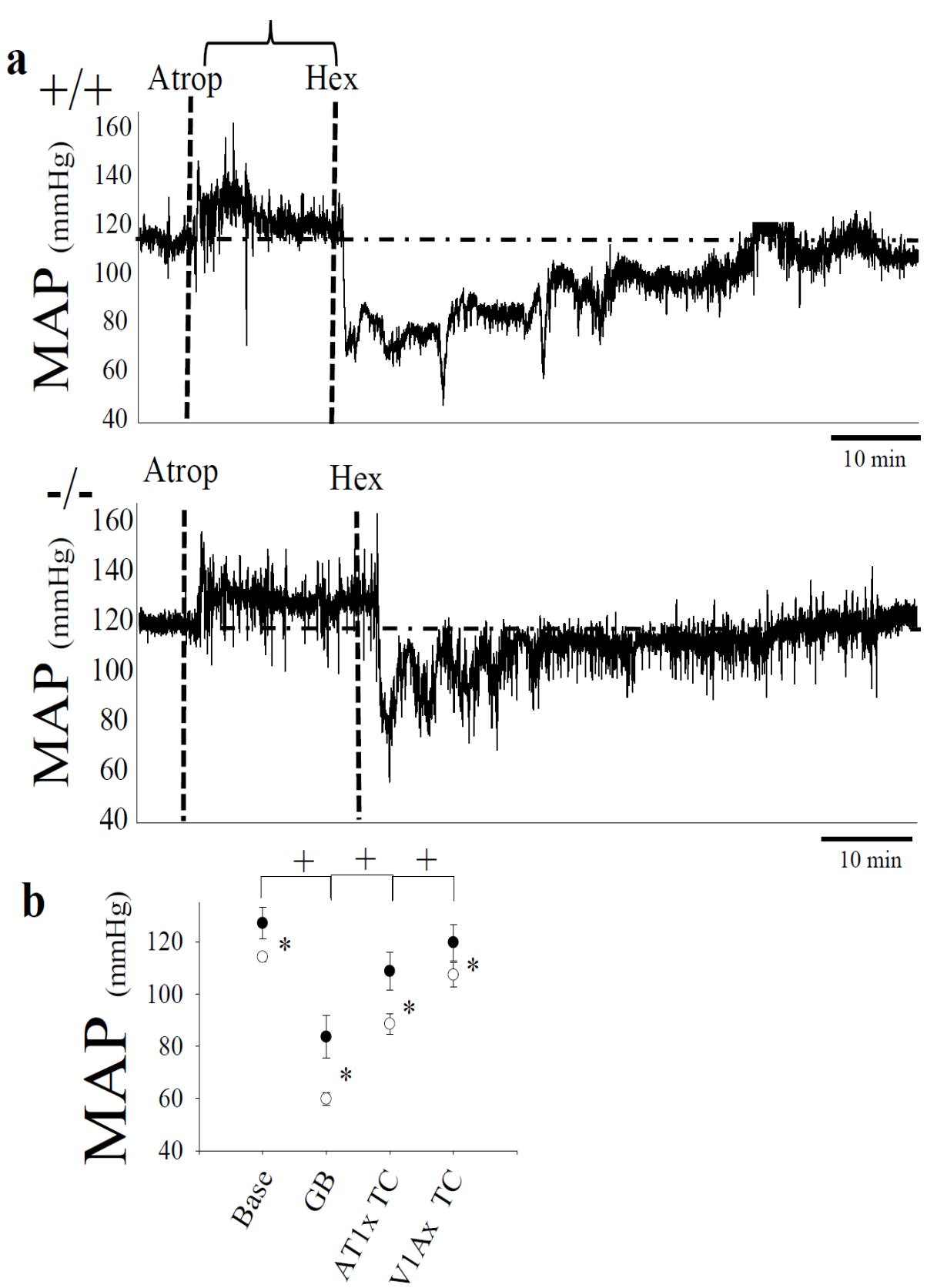

Figure 4.5: a) Shown are example traces, in QW, of mean arterial pressure (MAP, mmHg) in a WT (top) and $\mathrm{TPH} 2-/-$ (bottom) rat, showing the recovery following (at black vertical dashed lines) to atropine methyl nitrate (atrop, i.v. $1 \mathrm{mg} / \mathrm{kg}$, Sigma Chemical) and hexamethonium (hex, i.v. 30mg/kg, Sigma Chemical). GB: full ganglionic blockade. b) Average raw responses shown in male TPH2/- (closed circles, $n=5$ ) and WT controls (open circles, $n=5$ ) at baseline, following GB (atrop + hex). Also shown is the recovery of MAP at specific time points at which we injected losartan (an AT1 receptor antagonist; AT1x TC) and Manning compound (a V1A receptor antagonist; V1Ax TC). See Figure 6 for the responses to these antagonists following GB. *: genotype $\mathrm{p}<0.05 ;+$ : effect of treatment $\mathrm{p}<0.05$. Data are mean values \pm standard error of the mean. 

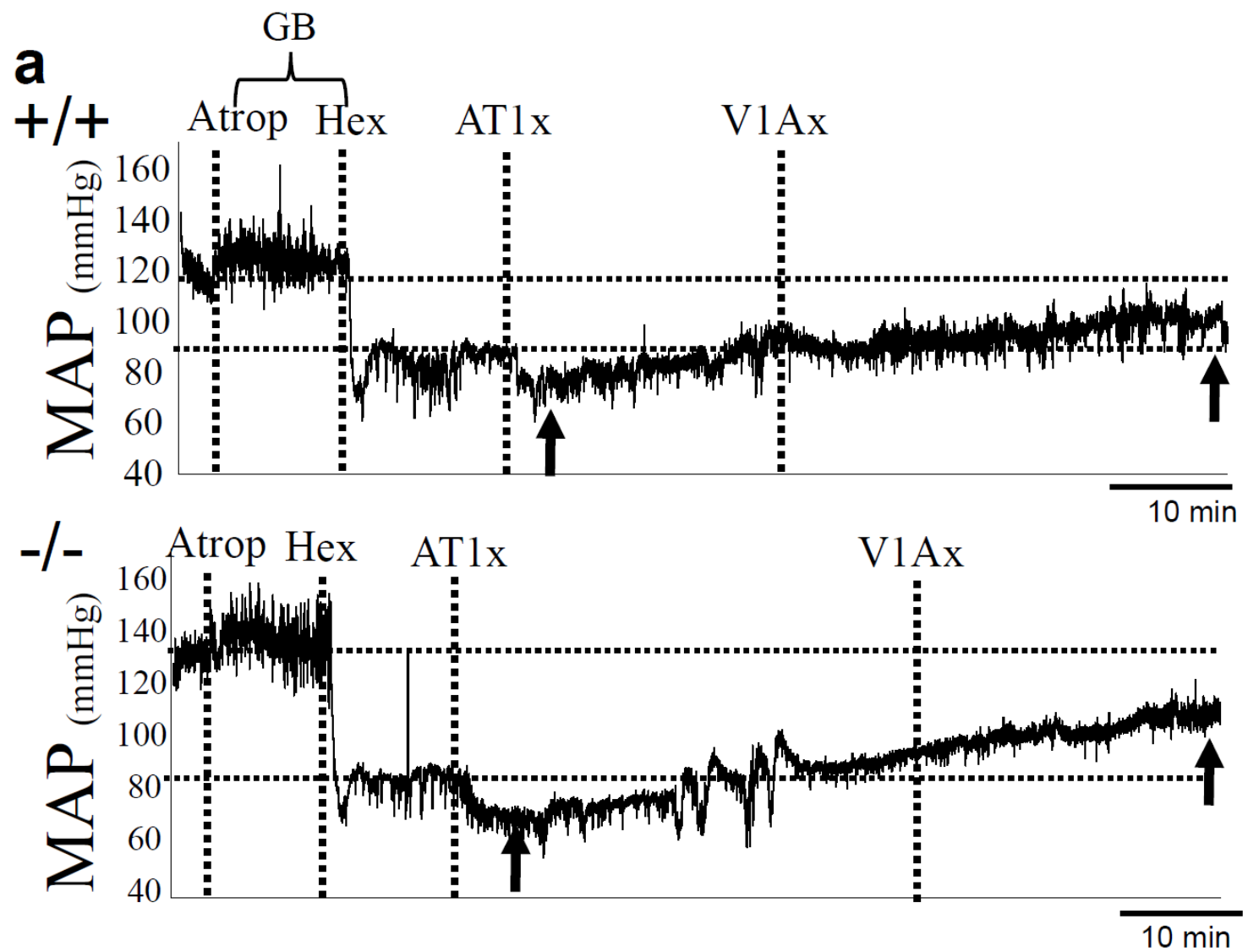

b

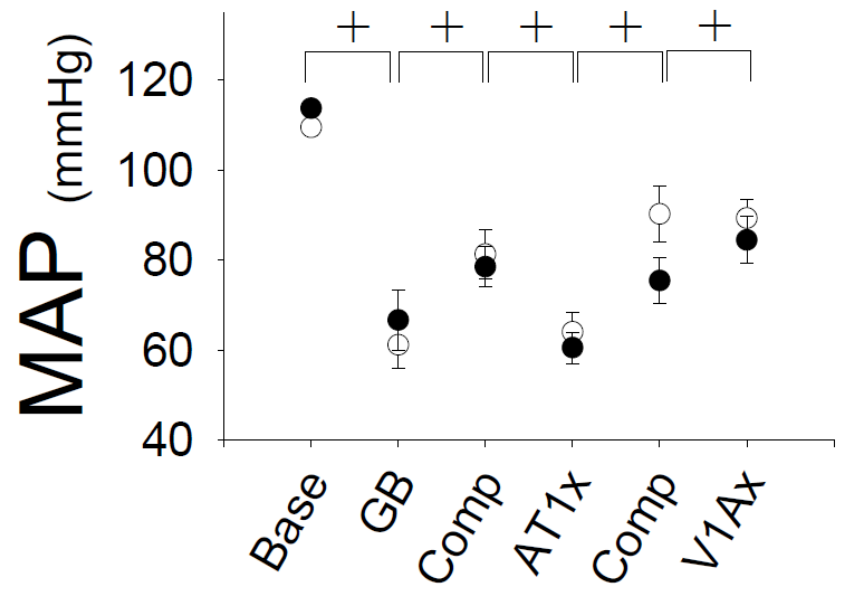

Figure 4.6: Central 5-HT contributes to angiotensin-mediated recovery of MAP following ganglionic blockade. a) Example mean arterial pressure (MAP, $\mathrm{mmHg}$ ) in a WT (top, $\mathrm{n}=6$ ) and $T P H 2$ - $/$ (bottom, $\mathrm{n}=6$ ) showing responses (at black vertical dashed lines) to atropine methyl nitrate (atrop, i.v., $1 \mathrm{mg} / \mathrm{kg}$ ), hexamethonium (hex, i.v., 30mg/kg), losartan (AT1x, i.v. $10 \mathrm{mg} / \mathrm{kg}$ ), and manning compound (V1Ax, i.v. $20 \mu \mathrm{g} / \mathrm{kg}$ ). Black arrows indicate where response to AT1x and V1Ax were assessed. b) Average MAP following atrop and hex (i.e. full ganglionic blockade, (GB)), and the responses to AT1x and V1Ax in male TPH2-/- (closed circle) and WT controls (open circle). + : effect of treatment $\mathrm{p}<0.05$. Data are mean values \pm standard error of the mean. 


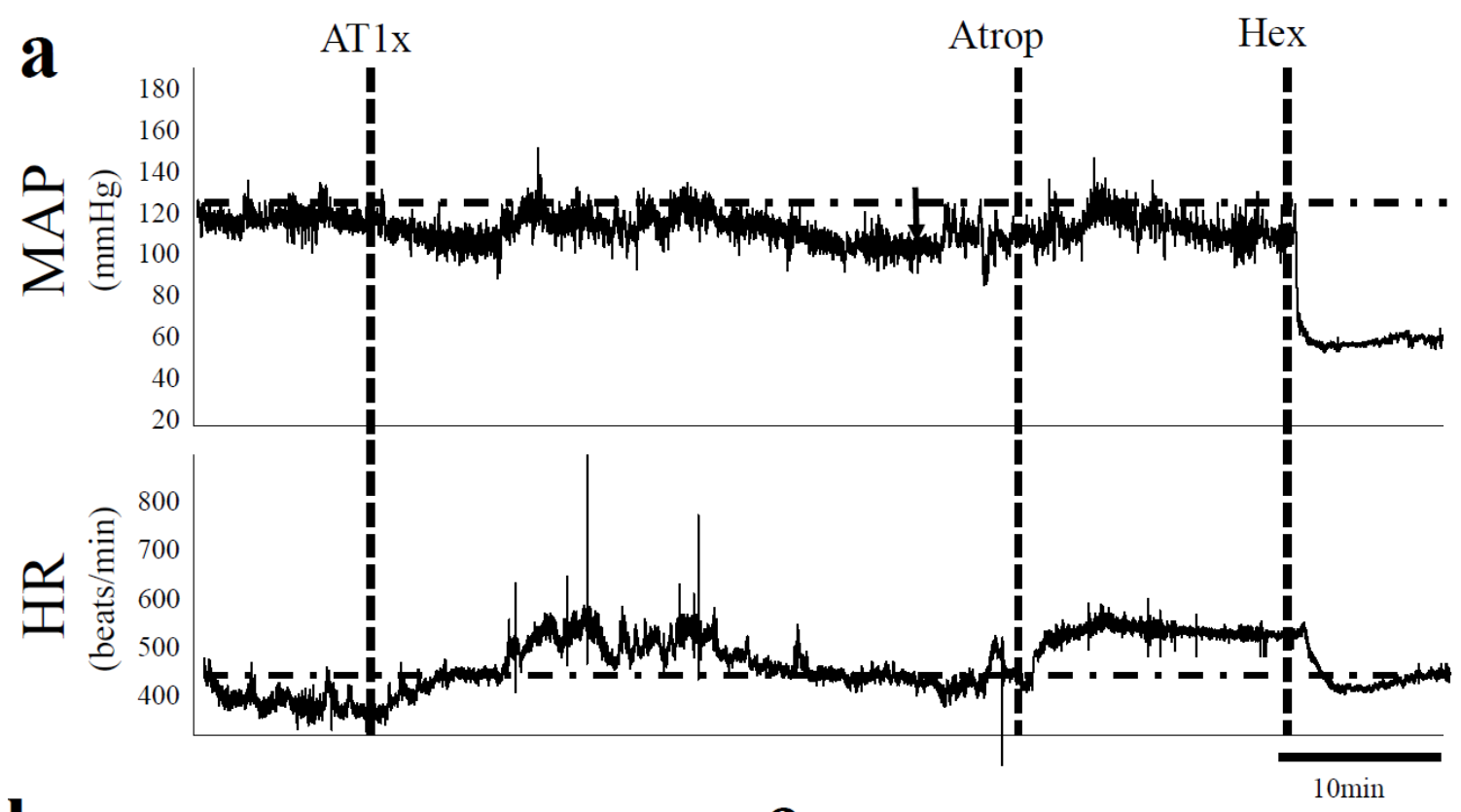

b

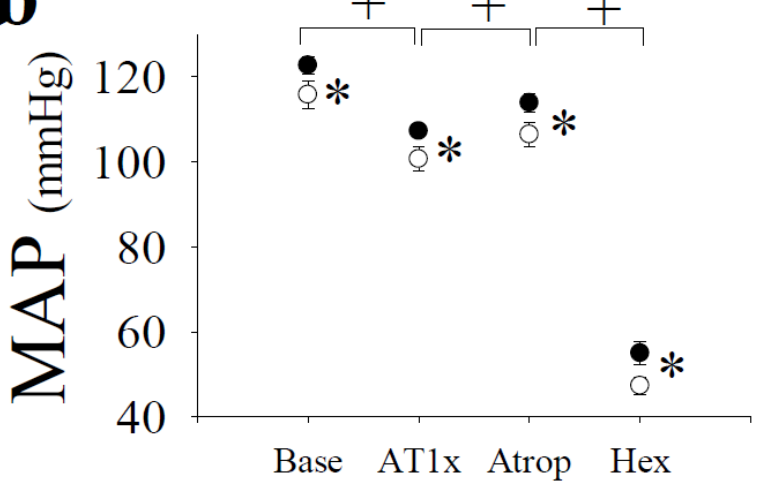

c

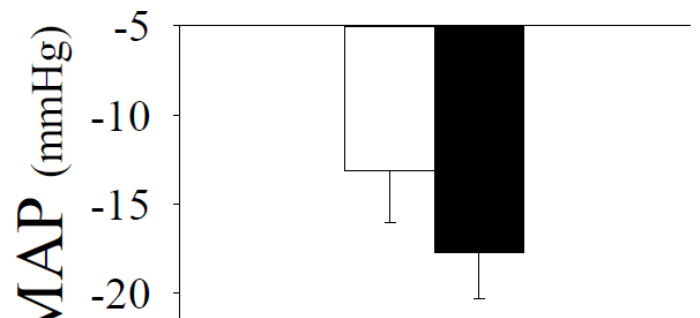

Figure 4.7: Angiotensin II does not contribute to the elevated ABP displayed by 5-HT deficient rats during resting conditions. a) Shown are typical responses (at black vertical dashed lines) of mean arterial pressure (MAP, mmHg, top) and heart rate (HR, beats/min, bottom) in a male WT rat to losartan (AT1x; i.v. $20 \mathrm{mg} / \mathrm{kg}$ ), atropine methyl nitrate (atrop; i.v. $1 \mathrm{mg} / \mathrm{kg}$ ) and hexamethonium (hex; i.v. 30mg/kg). b) Average responses shown (top) in male TPH2-/- (closed circles, $\mathrm{n}=6$ ) and WT controls (open circles, $\mathrm{n}=6$ ) during QW and following AT1x, atrop, and hex. Average $\triangle \mathrm{MAP}$ response to AT1x (bottom) in male TPH2-/- rats (closed bars, $n=6$ ) and WT (open bars, $n=6$ ). *: genotype $p<0.05 ;+$ : effect of treatment $p<0.05$. Data are mean values \pm standard error of the mean. 


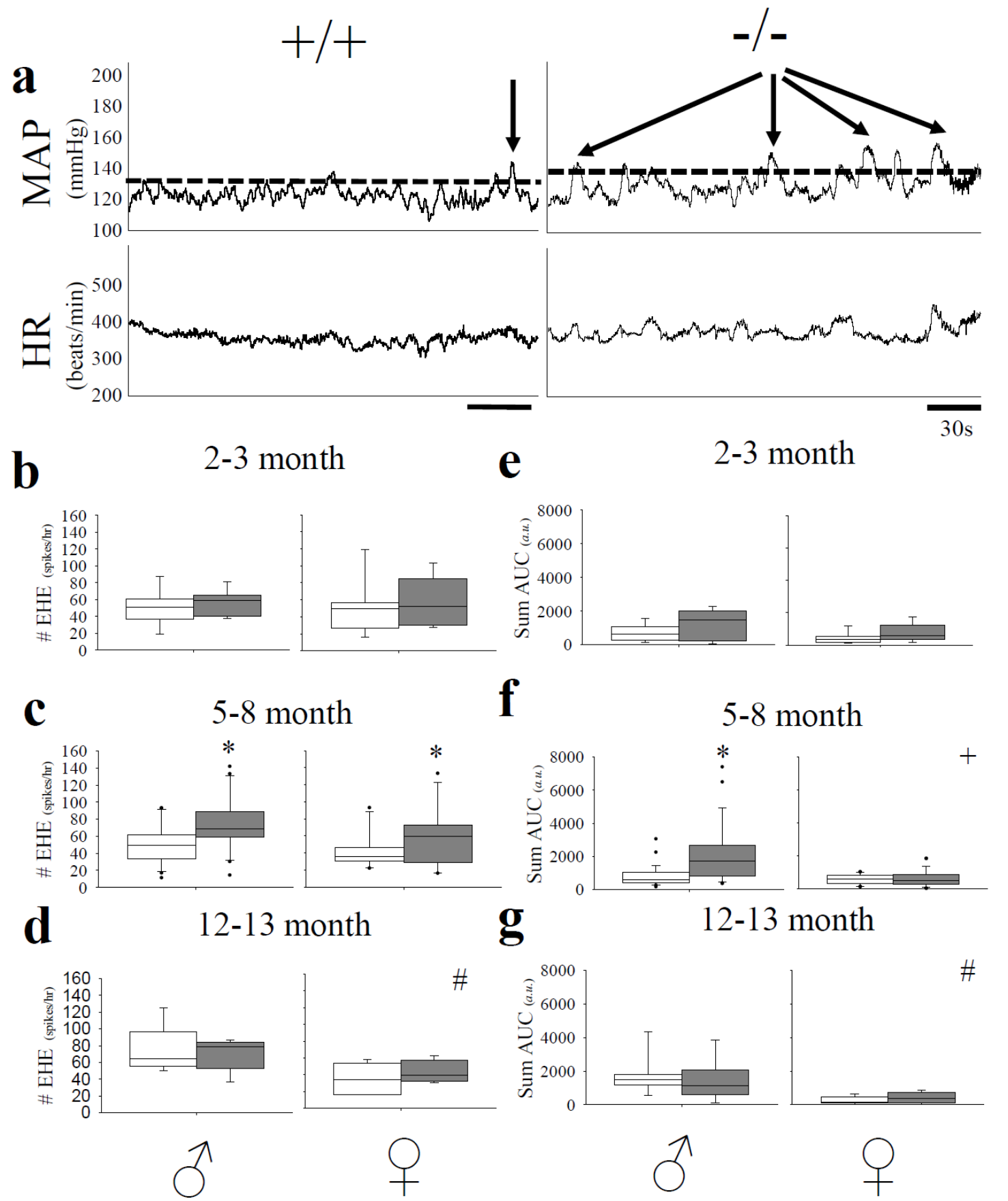

Figure 4.8: a) Example traces of mean arterial pressure (MAP, $\mathrm{mmHg}$, top) and heart rate (HR, beats/min, bottom) in a 5-8 month old male wildtype (+/+, left) and TPH2 knockout (-/-, right) rat showing an example of the criteria we used to assess "extreme hypertensive events (EHE's)" that occurred during REM sleep. Black dashed line shows the $20 \mathrm{mmHg}$ threshold, where any event that exceeds this line is considered an EHE, as indicated by the black-arrows. Number of EHE (spikes/hr) in male (left) and female (right) rats at b) 2-3 month old male $T P H 2-/$ - (dark gray, male $\mathrm{n}=7$, female $\mathrm{n}=6$ ) and WT controls (white, male $\mathrm{n}=8$, female $\mathrm{n}=9$ ); c) 6-8 month old $T P H 2-/$ - rats $($ male $=28$, female $\mathrm{n}=15)$ and WT controls (male $=30$, female $\mathrm{n}=12)$; and d) 12-13 month old $T P H 2-/$ (dark gray, male $n=6$, female $n=4$ ) and WT controls (white, male $n=9$, female $\mathrm{n}=7$ ). SumAUC in e) 2-3 month; f) 6-8 month; and g) 12-13 month old male (left) and female (right) rats. *: genotype $\mathrm{p}<0.05$; \#: sex $\mathrm{p}<0.005 ;+$ : genotype* sex Interaction $\mathrm{p}<0.05$. 


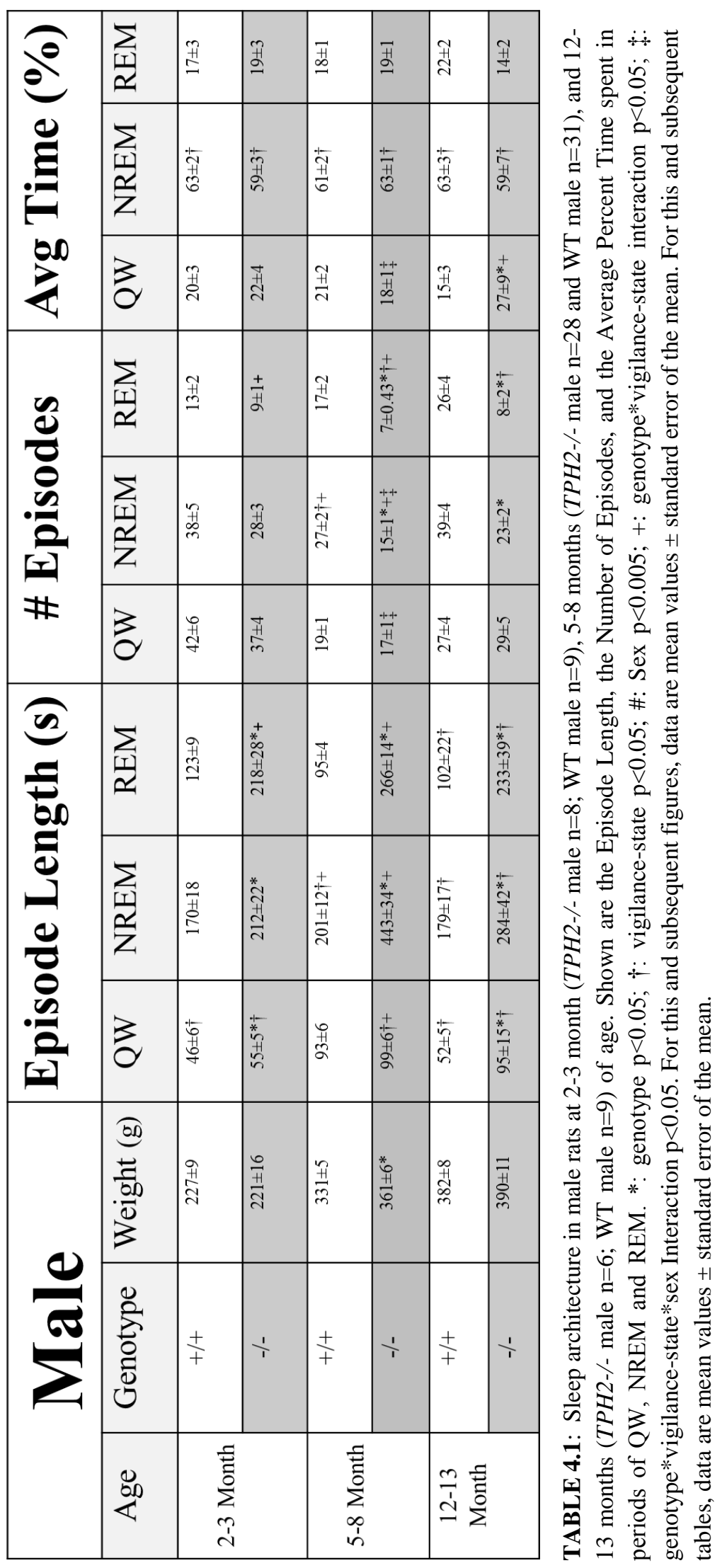




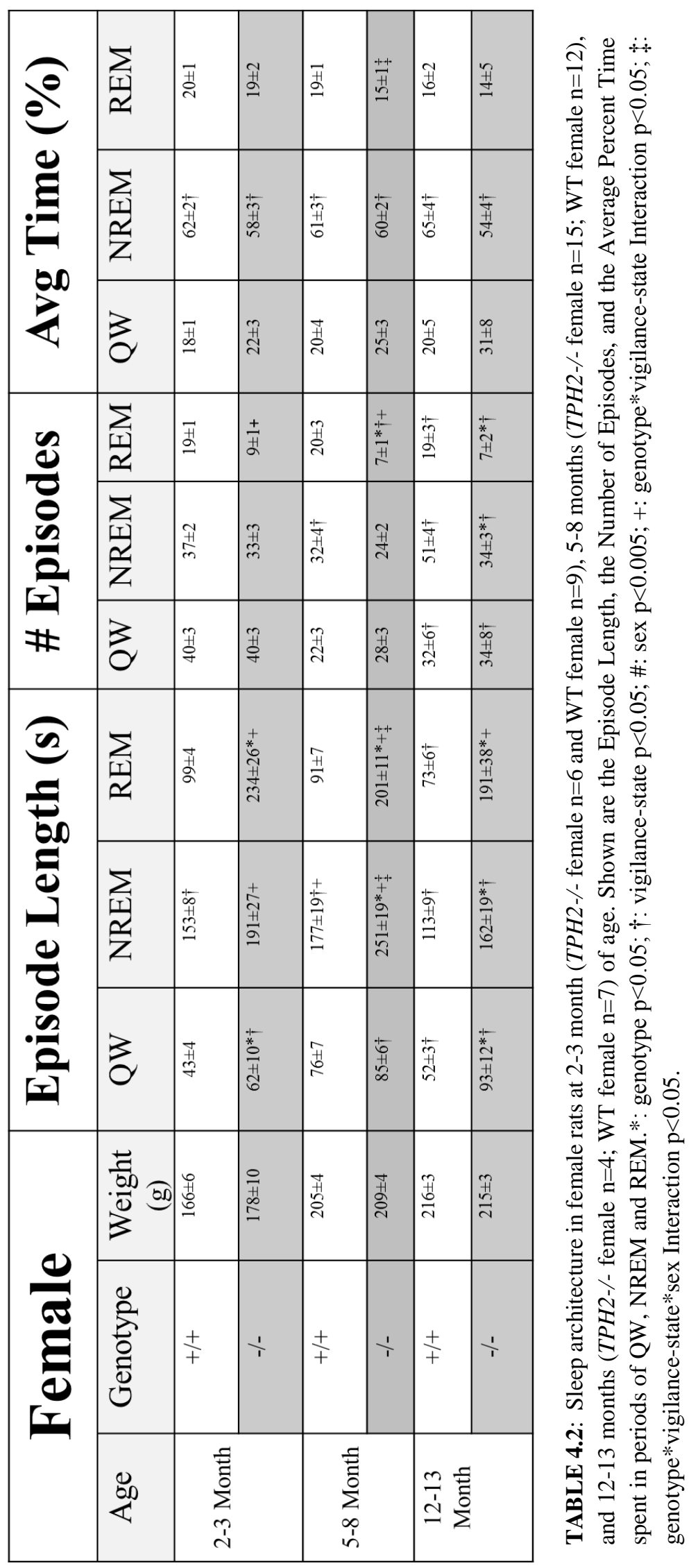




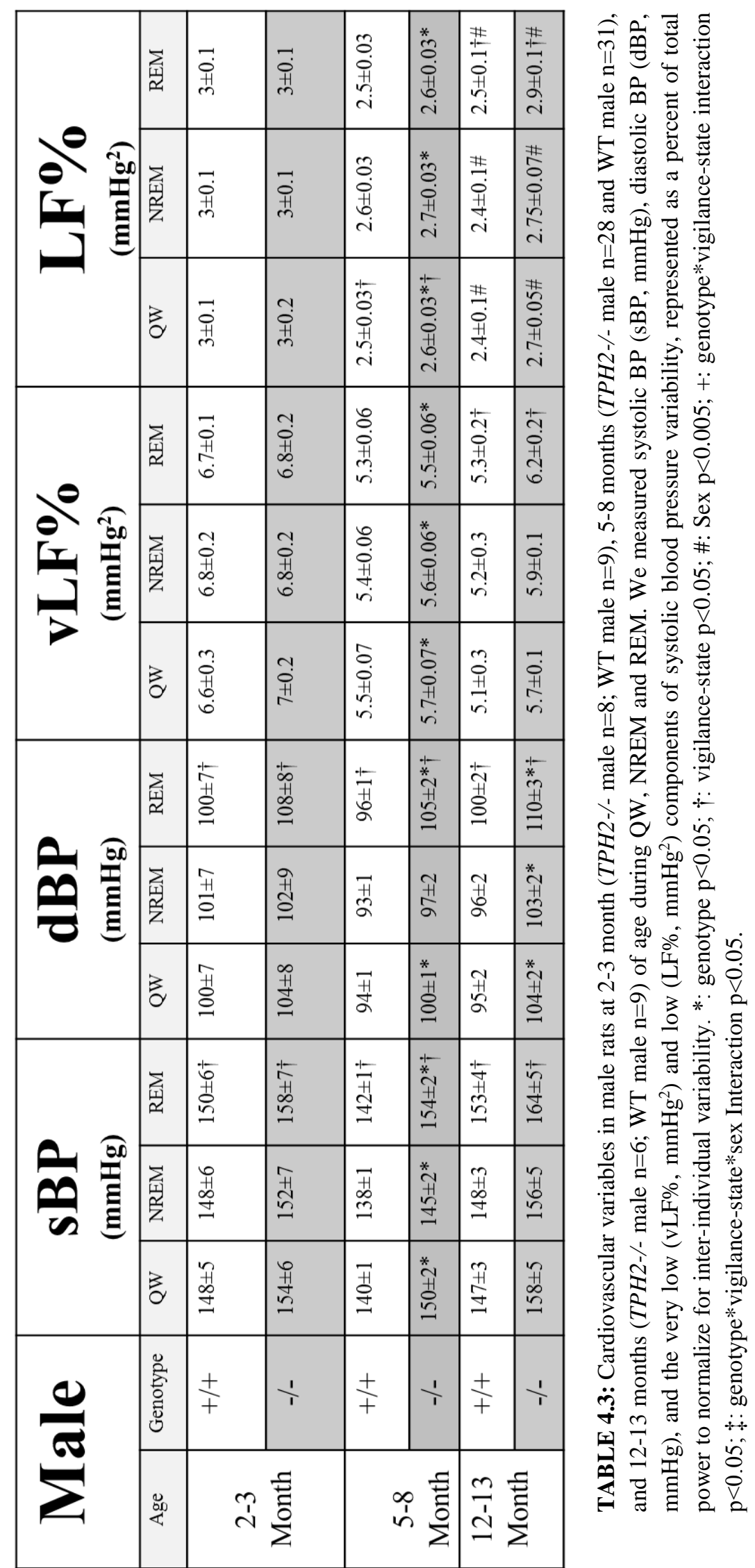




\begin{tabular}{|c|c|c|c|c|c|c|c|}
\hline \multirow{3}{*}{ dे } & 噇 & 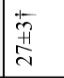 & $\mid \begin{array}{l}\bar{\pi} \\
\bar{\pi}\end{array}$ & $\begin{array}{l}\bar{y} \\
\text { 㬟 }\end{array}$ & $\begin{array}{l}\bar{y} \\
\stackrel{y}{g}\end{array}$ & 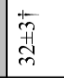 & 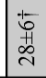 \\
\hline & $\begin{array}{l}\bar{y} \\
\frac{1}{2} \\
\end{array}$ & $\begin{array}{l}\text { 葛 } \\
=\end{array}$ & 葛 & $\vec{A}$ & $\frac{\vec{H}}{2}$ & 苦 & 吾 \\
\hline & $\nexists$ & $\begin{array}{l}\vec{H} \\
\text { 严 }\end{array}$ & $\vec{\beth}$ & 竞 & 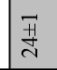 & 苦 & 苦 \\
\hline \multirow{3}{*}{ 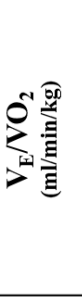 } & $\sum$ & 苦 & $\mid \begin{array}{l} \pm \\
\text { 売 } \\
\text { 苟 }\end{array}$ & 売 & $\underset{\sim}{\stackrel{\vec{N}}{N}}$ & $\begin{array}{l}\text { त्र } \\
\text { तै } \\
\text { a }\end{array}$ & 离 \\
\hline & 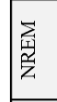 & 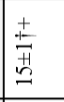 & 芩 & 晜 & 落 & 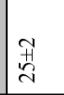 & $\frac{\pi}{d}$ \\
\hline & 辛 & 莫 & 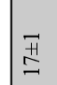 & $\underset{⿱}{\stackrel{H}{\sim}}$ & 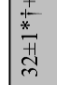 & $\begin{array}{l}\text { Iy } \\
\text { 吾 }\end{array}$ & 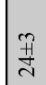 \\
\hline \multirow{3}{*}{$\overbrace{}^{0 \text { 施 }}$} & 产 & $\begin{array}{l}\text { 蒂 } \\
\frac{7}{0}\end{array}$ & $\begin{array}{l}\text { 萎 } \\
\text { 范 }\end{array}$ & $\frac{\#}{N}$ & $\frac{\pi}{\sim}$ & 輁 & 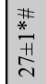 \\
\hline & \begin{tabular}{|l}
$\frac{\pi}{10}$ \\
$\frac{1}{2}$ \\
\end{tabular} & \begin{tabular}{|l} 
崂 \\
总 \\
\end{tabular} & 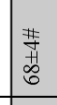 & 苦 & \begin{tabular}{|l}
$\vec{H}$ \\
ते
\end{tabular} & \begin{tabular}{|l} 
崂 \\
贲 \\
\end{tabular} & 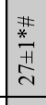 \\
\hline & $\nexists$ & \begin{tabular}{|l}
+4 \\
贲 \\
6 \\
\end{tabular} & 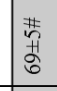 & 喜 & 辛 & 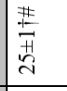 & 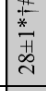 \\
\hline \multirow{3}{*}{$>$ > } & 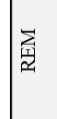 & 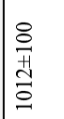 & 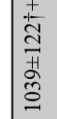 & 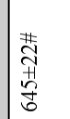 & 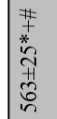 & 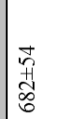 & 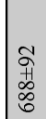 \\
\hline & 产 & 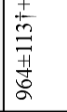 & 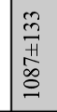 & $\begin{array}{l}\text { 葶 } \\
\text { 苦 } \\
\text { 6 }\end{array}$ & 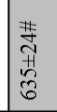 & 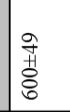 & $\begin{array}{l}8 \\
0 \\
11 \\
16 \\
6\end{array}$ \\
\hline & 3 & 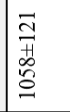 & $\begin{array}{l}\frac{\partial}{7} \\
\frac{\partial}{\partial} \\
\stackrel{\partial}{=}\end{array}$ & 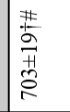 & 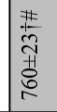 & 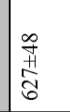 & $\begin{array}{l}8 \\
\frac{0}{40} \\
\frac{0}{0}\end{array}$ \\
\hline \multirow{3}{*}{ ? } & 屋 & 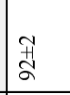 & \begin{tabular}{|l}
$\frac{t}{*}$ \\
$\frac{*}{+}$ \\
$\frac{H}{\infty}$ \\
\end{tabular} & 菩 & 辛 & 袰 & 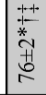 \\
\hline & $\begin{array}{l}\overline{\mathbf{z}} \\
\bar{z} \\
\end{array}$ & 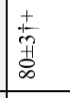 & $\begin{array}{l}\vec{\forall} \\
\infty \\
\infty\end{array}$ & 苦 & $\begin{array}{l} \pm \\
\stackrel{ \pm}{*} \\
\stackrel{ \pm}{*}\end{array}$ & $\begin{array}{l}\text { 范 } \\
\end{array}$ & त्र \\
\hline & əे & $\frac{\#}{\infty}$ & 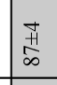 & 芩 & 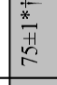 & 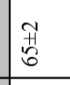 & की \\
\hline \multirow{3}{*}{$>$ P } & 端 & 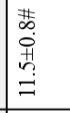 & 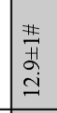 & 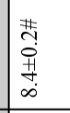 & 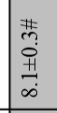 & 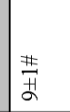 & 帮 \\
\hline & 产 & 莘 & $\begin{array}{l}\text { 菩 } \\
\text { 莡 } \\
\text { I }\end{array}$ & 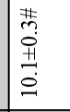 & 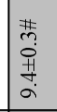 & 鿓 & 素莫 \\
\hline & 竎 & 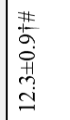 & 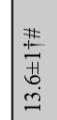 & 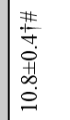 & 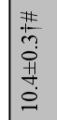 & 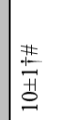 & 鋀 \\
\hline \multirow{2}{*}{$\underset{\sum}{2}$} & 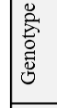 & $\stackrel{t}{+}$ & $\frac{1}{1}$ & $\stackrel{t}{+}$ & $\frac{1}{1}$ & $\stackrel{+}{+}$ & $\frac{1}{1}$ \\
\hline & 喿 & \multicolumn{2}{|c|}{ 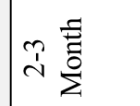 } & \multicolumn{2}{|c|}{ 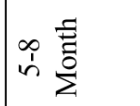 } & \multicolumn{2}{|c|}{ 恶 } \\
\hline
\end{tabular}

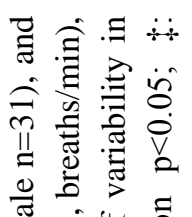

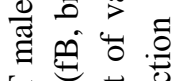

这泀

류:

六

II

芶

i $\frac{1}{5} \frac{50}{5}$

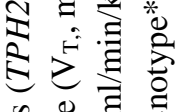

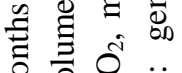

节苛

乩豞出

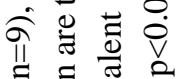

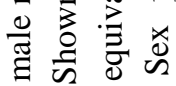

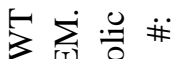

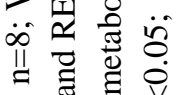

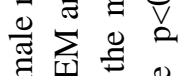

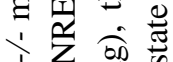

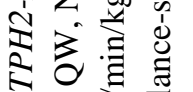

일

言

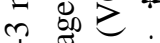

ஸे पँ

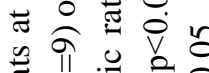

苛 II

光芯芯 芯

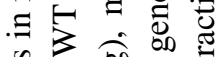

峁

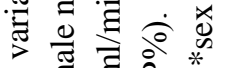

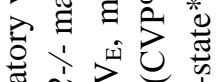

.

๙ิ

in

되율류

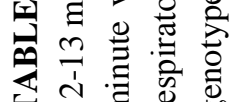




\begin{tabular}{|c|c|c|c|c|c|c|c|}
\hline \multirow{3}{*}{ 己े } & 画 & $\underset{\mathrm{H}}{\mathrm{H}}$ & 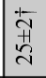 & 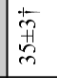 & $\begin{array}{l}1 \\
\text { जै } \\
\text { on }\end{array}$ & 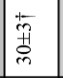 & \begin{tabular}{|l} 
离 \\
芴
\end{tabular} \\
\hline & 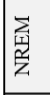 & $\vec{I}$ & $\stackrel{I}{I}$ & $\frac{\pi}{\pi}$ & $\begin{array}{l}\overrightarrow{+} \\
\stackrel{\vec{x}}{-}\end{array}$ & $\begin{array}{l}\text { 恿 } \\
-1\end{array}$ & $\stackrel{\oplus}{\Xi}$ \\
\hline & 8 & \begin{tabular}{|l}
$n$ \\
0 \\
0 \\
0 \\
0
\end{tabular} & $\begin{array}{l}\text { 吾 } \\
-\end{array}$ & 萗 & $\frac{\pi}{N}$ & 弯 & $\frac{\vec{H}}{N}$ \\
\hline \multirow{3}{*}{ 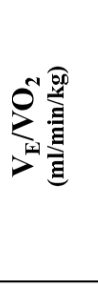 } & 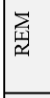 & 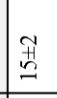 & 苞 & $\frac{\pi}{m}$ & $\begin{array}{l}\bar{H} \\
\stackrel{\lambda}{2}\end{array}$ & \begin{tabular}{|l|l} 
\\
in \\
0
\end{tabular} & 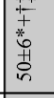 \\
\hline & 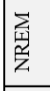 & 苔 & 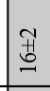 & 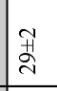 & 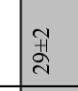 & â & 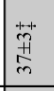 \\
\hline & $\ddot{z}$ & \begin{tabular}{|l} 
\pm \\
\\
\\
\\
\end{tabular} & 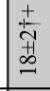 & 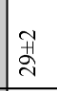 & $\mid \begin{array}{l} \pm \\
\frac{ \pm}{m} \\
\frac{\pi}{m}\end{array}$ & $\frac{\pi}{m}$ & 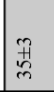 \\
\hline \multirow{3}{*}{$\rho^{0}$} & $\mid \bar{x}$ & \begin{tabular}{|l}
$\overline{0}$ \\
$\overline{+}$ \\
$\infty$ \\
\end{tabular} & 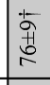 & \begin{tabular}{|c} 
芦 \\
m
\end{tabular} & $\frac{\pi}{m}$ & $\begin{array}{l}7 \\
⿱ \\
m\end{array}$ & $\frac{\vec{H}}{m}$ \\
\hline & 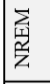 & 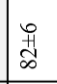 & $\begin{array}{l}0 \\
\infty \\
\infty \\
\infty\end{array}$ & 吾 & 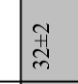 & $\begin{array}{l}\text { 苦 } \\
\text { n }\end{array}$ & $\frac{\nexists}{m}$ \\
\hline & 咅 & $\begin{array}{l}0 \\
\text { 落 } \\
\end{array}$ & \begin{tabular}{l}
0 \\
0 \\
\hdashline \\
\end{tabular} & 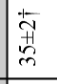 & $\begin{array}{l}1+1 \\
\text { ल } \\
m\end{array}$ & 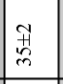 & 岱 \\
\hline \multirow{3}{*}{$>$ 章 } & $\mid \bar{i}$ & 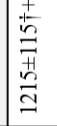 & $\underset{\Xi}{\bar{J}}$ & 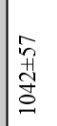 & 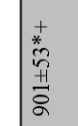 & 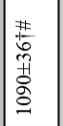 & 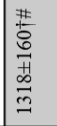 \\
\hline & 章 & \begin{tabular}{|l} 
Oे \\
$\stackrel{H}{H}$ \\
$\stackrel{\Xi}{\Xi}$ \\
\end{tabular} & సે & 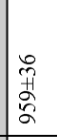 & $\begin{array}{l}\overline{5} \\
\bar{y} \\
y \\
y\end{array}$ & 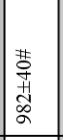 & 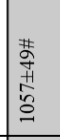 \\
\hline & $\vec{z}$ & $\begin{array}{l}\text { 志 } \\
\stackrel{+}{ \pm} \\
\Xi \\
\end{array}$ & 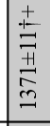 & 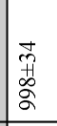 & $\mid \begin{array}{l}\frac{ \pm}{5} \\
\frac{1}{5} \\
\frac{1}{0} \\
\frac{0}{0} \\
\end{array}$ & 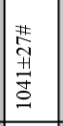 & 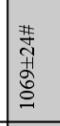 \\
\hline \multirow{3}{*}{ 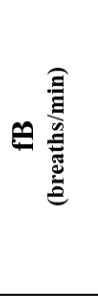 } & $\mid \begin{array}{l}\overline{\mid} \\
\Sigma\end{array}$ & 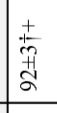 & \begin{tabular}{l}
$*$ \\
\multirow{1}{*}{} \\
$\vdots$ \\
$\infty$ \\
$\infty$
\end{tabular} & 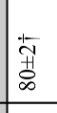 & 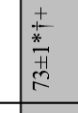 & 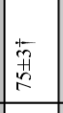 & 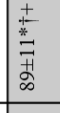 \\
\hline & 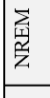 & $\begin{array}{l}0 \\
0 \\
\end{array}$ & 苦 & 㺃 & 藏 & $\begin{array}{l}\text { 悉 } \\
\end{array}$ & 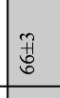 \\
\hline & 总 & 容 & $\underset{\infty}{n}$ & 害 & \begin{tabular}{|l} 
苞 \\
总
\end{tabular} & 㺃 & $\frac{7}{7}$ \\
\hline \multirow{3}{*}{$>$ 窵 } & 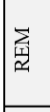 & \begin{tabular}{|l} 
Oे \\
严 \\
-9
\end{tabular} & 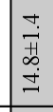 & \begin{tabular}{|l}
0 \\
\\
în \\
İ \\
\end{tabular} & \begin{tabular}{|l}
0 \\
0 \\
11 \\
1 \\
\\
\end{tabular} & 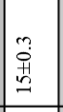 & \begin{tabular}{|l}
0 \\
Oे \\
ग्रे
\end{tabular} \\
\hline & 童 & 吾 & 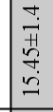 & \begin{tabular}{|l}
0 \\
\\
$\dot{1}$ \\
$\dot{I}$ \\
\end{tabular} & $\begin{array}{l}r \\
\dot{y} \\
\dot{y} \\
\dot{I}\end{array}$ & $\begin{array}{l}0 \\
0 \\
0 \\
0 \\
2\end{array}$ & $\begin{array}{l}3 \\
0 \\
0 \\
0 \\
0\end{array}$ \\
\hline & 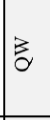 & 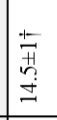 & 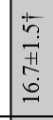 & 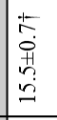 & 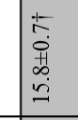 & 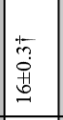 & 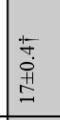 \\
\hline \multirow{2}{*}{$\underset{0}{\frac{0}{0}}$} & 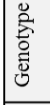 & $\stackrel{+}{+}$ & $\frac{1}{T}$ & $\stackrel{+}{+}$ & $\frac{1}{1}$ & $\frac{+}{+}$ & $\frac{1}{1}$ \\
\hline & 总 & \multicolumn{2}{|c|}{$\stackrel{\dot{\sim}}{\dot{0}}$} & \multicolumn{2}{|c|}{$\begin{array}{l}\infty \\
\infty \\
i \\
i \\
\Sigma \\
\Sigma\end{array}$} & \multicolumn{2}{|c|}{ m } \\
\hline
\end{tabular}

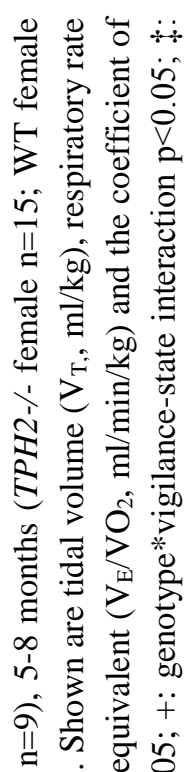

过

ए

$3 \sum_{i} \pm$

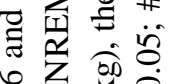

II

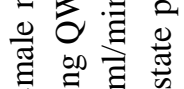

है ह हो क

I

疍

$\forall$ 㟧 ?

吾吾语

\& $\frac{\pi}{0} \dot{v}$

के छㄹ

क

卷 3

ป II

을

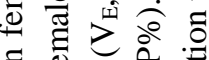

$\Xi \stackrel{2}{2}$

बे

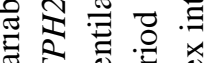

స छ

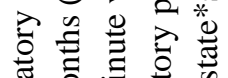

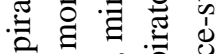

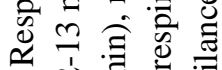

$\ddot{0} \stackrel{\Xi}{\square}$

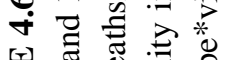

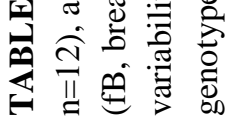


a

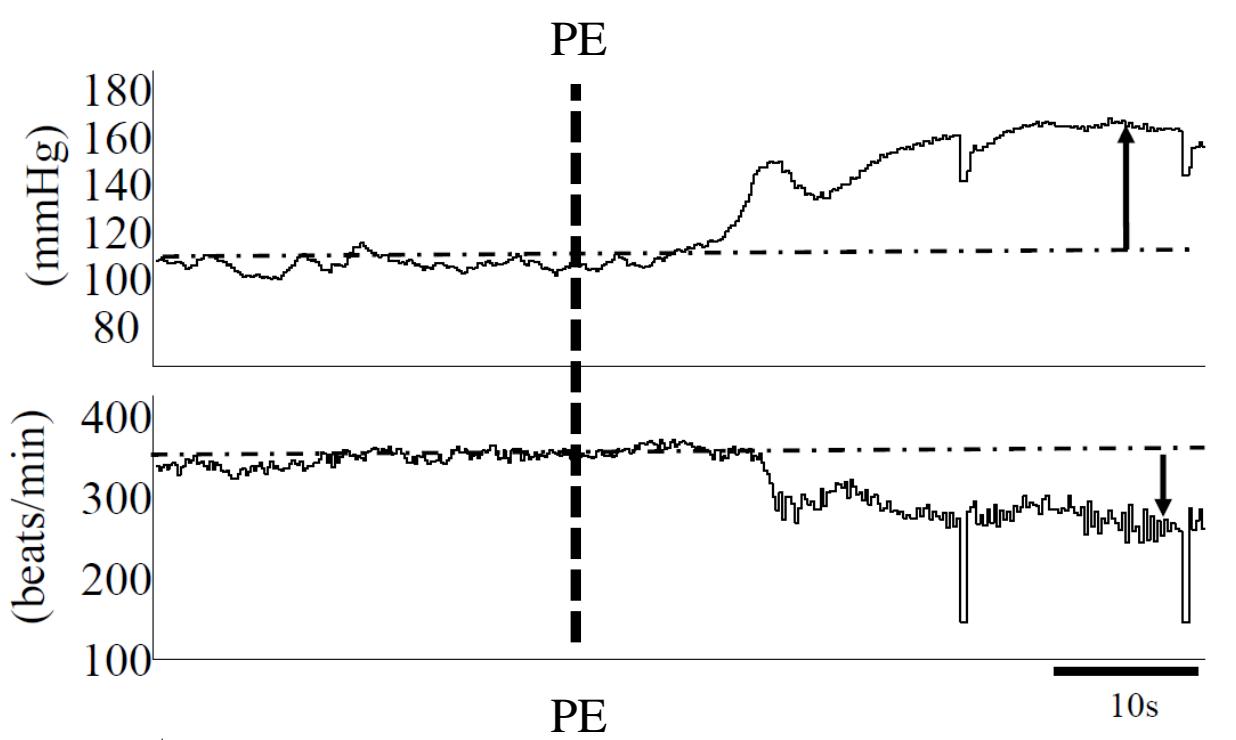

b

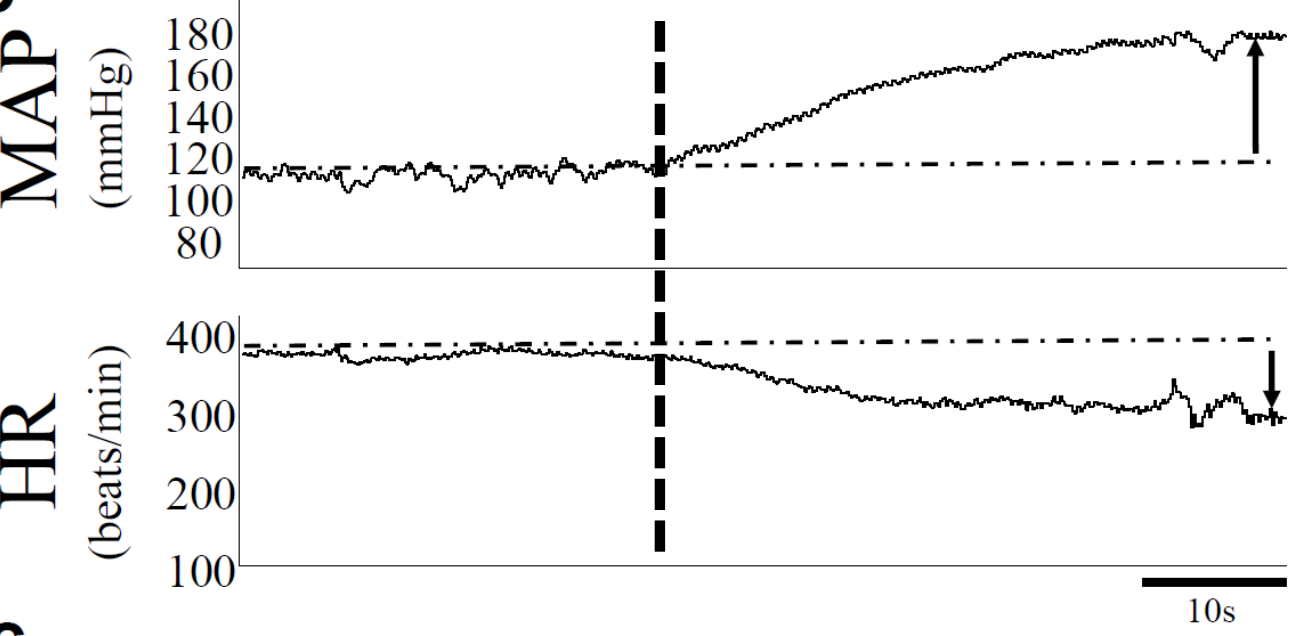

C

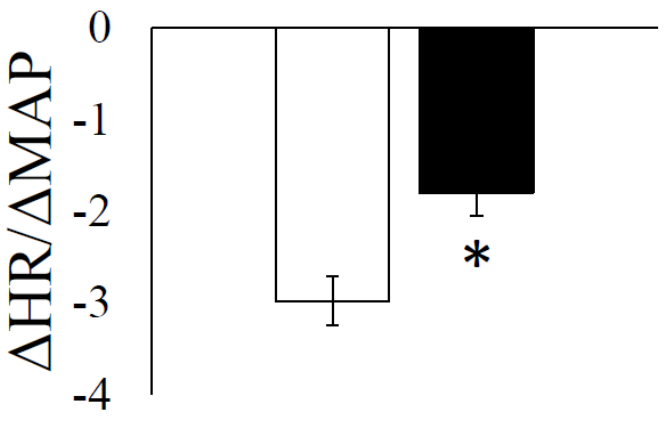

Supplemental Figure 4.9: Example mean arterial pressure (MAP, mmHg, top) and heart rate (HR, beats/min, bottom) in a WT (a) and TPH2-/- (b) rats showing the responses to phenylephrine at vertical black dashed line (PE, 20ul of $1 \mathrm{mg} / \mathrm{ml}$ ). Our goal was to infuse PE slowly while monitoring the increase in ABP within a range of $50-60 \mathrm{mmHg}$ over a period of $45-60$ seconds. Baroreflex reactivity was assessed to determine the relationship between peak changes (black arrows) in the $\triangle \mathrm{HR}$ for a given $\triangle \mathrm{MAP}$ $(\triangle \mathrm{HR} / \triangle \mathrm{MAP})$. c) Average $\triangle \mathrm{HR} / \triangle \mathrm{MAP}$ in $T P H 2-/$ (closed bars, $\mathrm{n}=2,6 \mathrm{PE}$ responses) and WT (open bars, $\mathrm{n}=2,8 \mathrm{PE}$ responses) rats to $\mathrm{PE}$. 


\section{a Control}

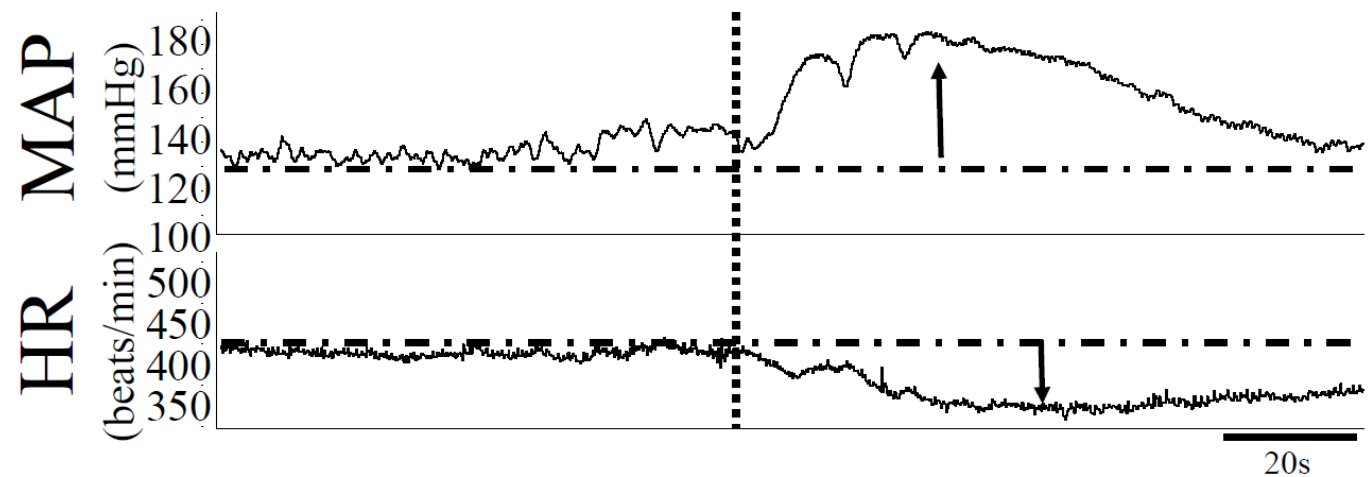

\section{b Losartan AngII}

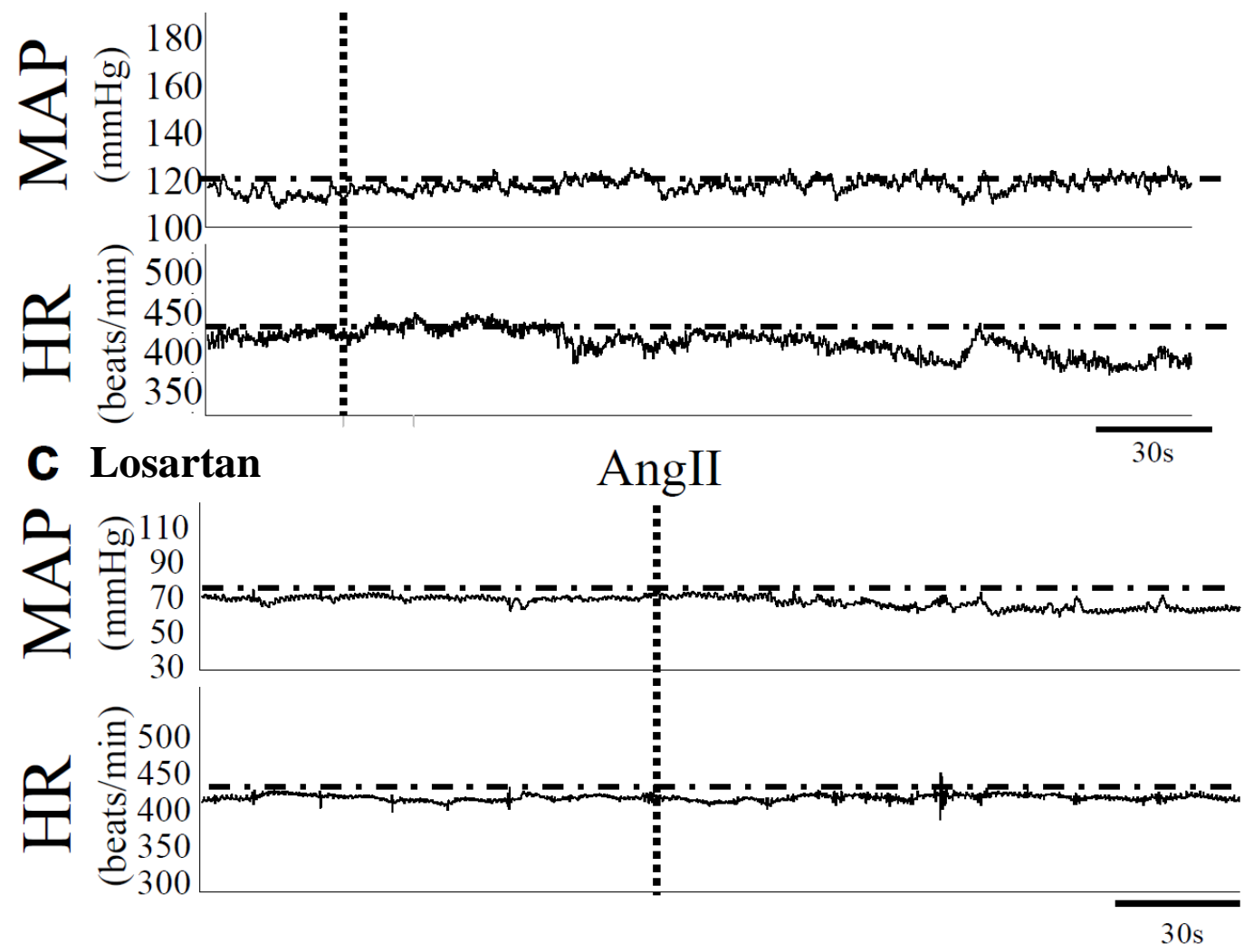

Supplemental Figure 4.10: In a separate group of rats not treated with ganglionic blockade, we confirmed the length of time that losartan abolished the pressure and HR responses to Angiotensin II (Ang II, 25ng/kg). Shown are example MAP (mmHg, top) and HR (beats/min, bottom) traces in a WT rat before (a) and after (b) losartan (10mg/kg, i.v.). In (c), shown are example MAP (mmHg, top) and HR (beats/min, bottom) in a WT rat from Group 3 rats showing the responses (at black vertical dashed lines) to Ang II, showing that the blockade of AT1 receptors with Losartan was complete. 


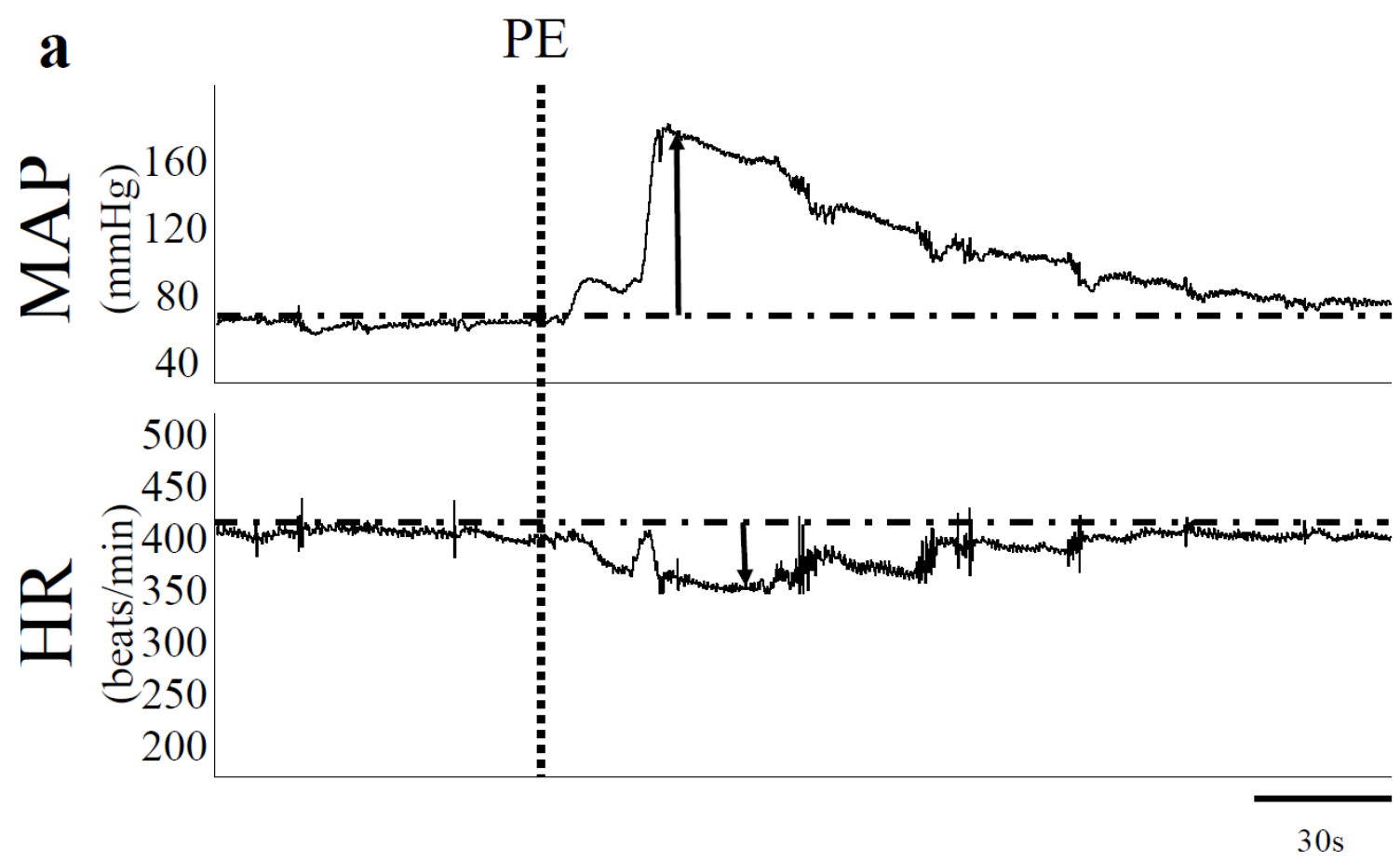

b

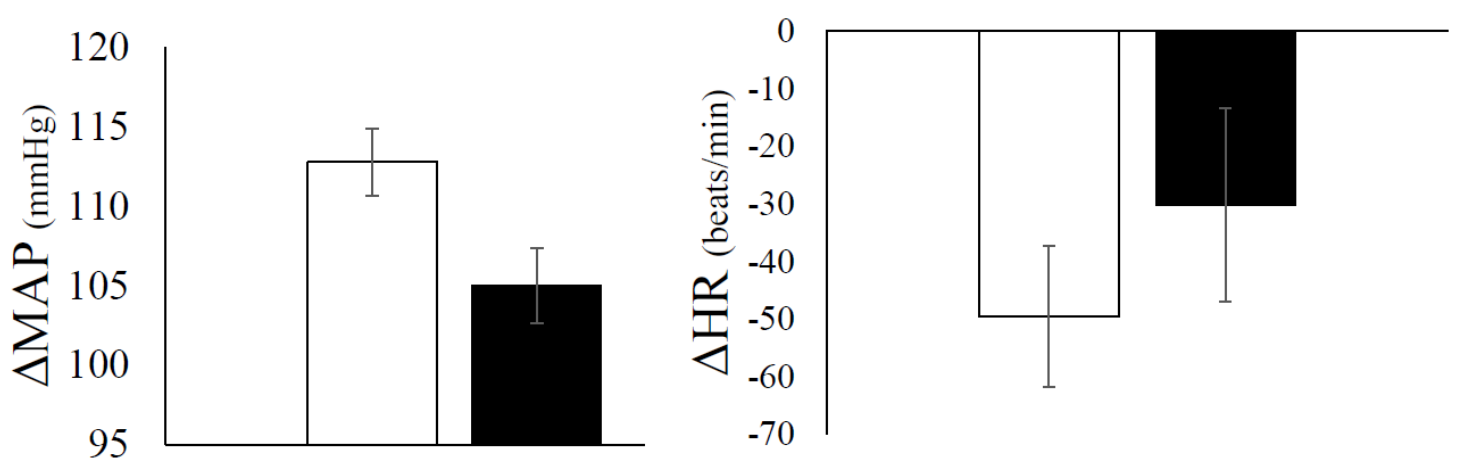

Supplemental Figure 4.11: a) Example mean arterial pressure (MAP, $\mathrm{mmHg}$, top) and heart rate (HR, beats/min, bottom) in a WT rat showing the responses (at black vertical dashed lines) to phenylephrine (PE, i.v. $10 \mu \mathrm{g} / \mathrm{kg}$ ). b) Average MAP (left, $\mathrm{mmHg}$ ) and HR (right, beats/min) in TPH2-/- (closed bars, $\mathrm{n}=5$ ) and WT (open bars, $\mathrm{n}=5$ ) rats to $\mathrm{PE}$ (i.v., 25ng/kg). 


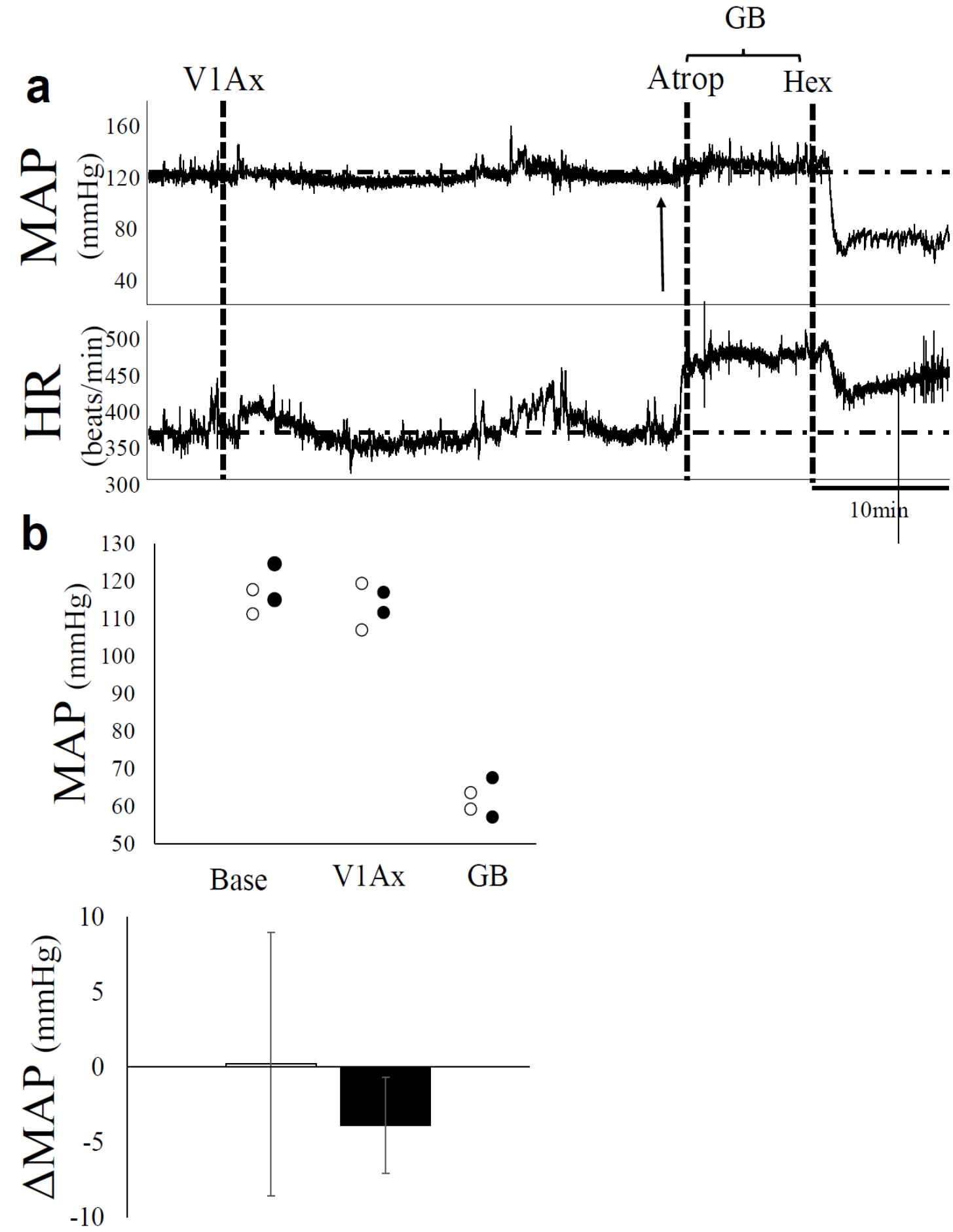

Supplemental Figure 4.12: a) Example traces of mean arterial pressure (MAP, mmHg, top) and heart rate (HR, beats/min, bottom) in a WT rat showing the responses (at black vertical dashed lines) to Manning Compound (V1Ax; i.v. $20 \mu \mathrm{g} / \mathrm{kg}$, Sigma Chemical), atropine methyl nitrate (atrop; i.v. $1 \mathrm{mg} / \mathrm{kg}$ ) and hexamethonium (hex; i.v. 30mg/kg). b) top: MAP in TPH2-/- rats ( $\mathrm{n}=2$, closed dots) and WT controls $(\mathrm{n}=2$, open dots) at baseline, and following V1 Ax and ganglionic blockade (GB) with atrop (i.v. $1 \mathrm{mg} / \mathrm{kg}$ ) and hex (i.v. $30 \mathrm{mg} / \mathrm{kg}$ ). bottom: $\triangle \mathrm{MAP}$ response (black arrow) to V1Ax in male TPH2-/- rats (closed bars, $\mathrm{n}=2$ ) and WT (open bars, $n=2$ ). *: genotype $\mathrm{p}<0.05 ;+$ : effect of treatment $\mathrm{p}<0.05$. 


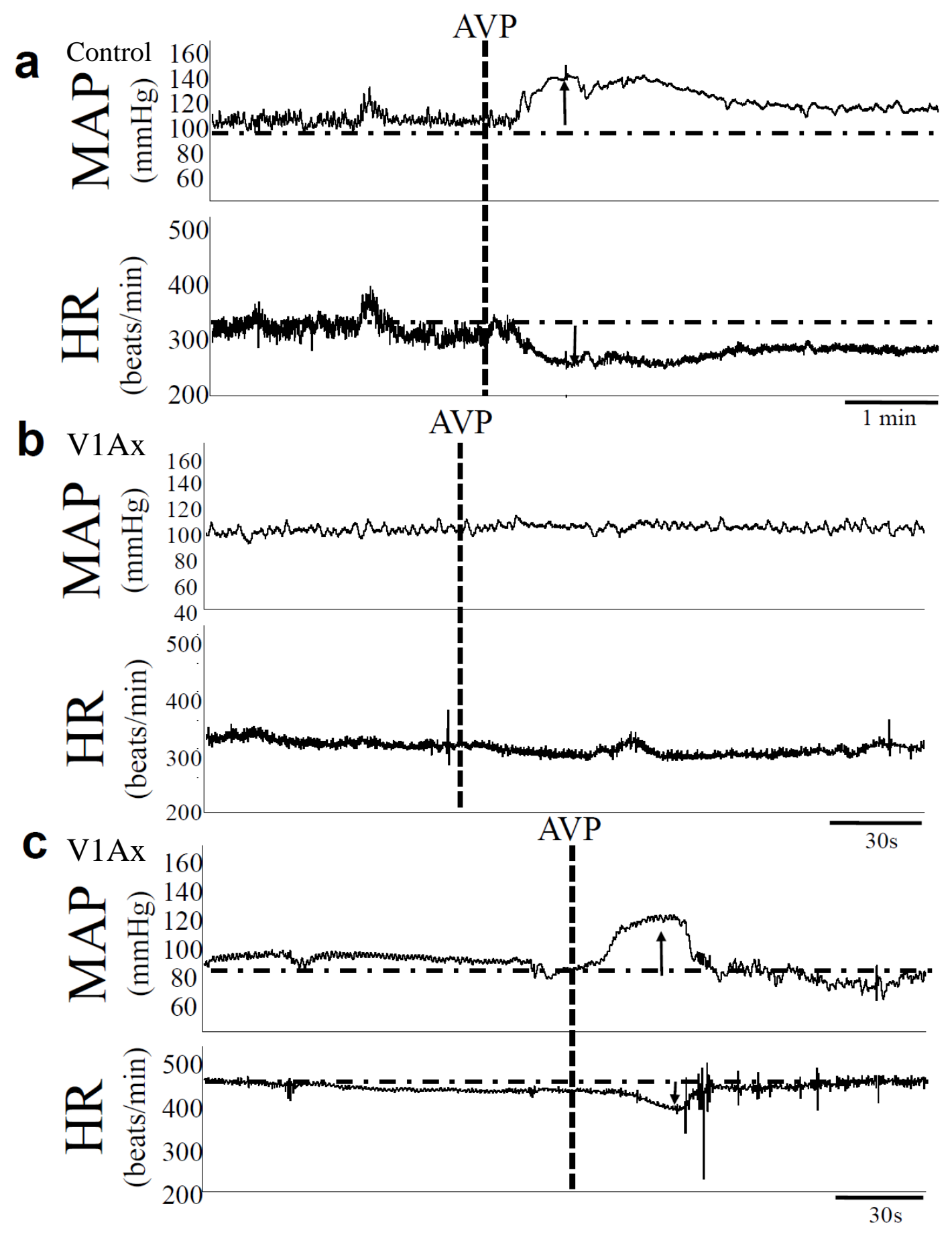

Supplemental Figure 4.13: In a separate group of rats not treated with ganglionic blockade, we confirmed

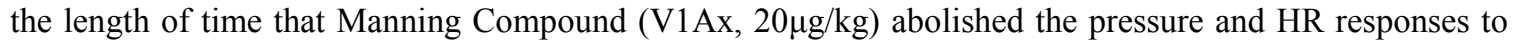
vasopressin (AVP, 20ng/kg). Shown are example MAP (mmHg, top) and HR (beats/min, bottom) traces in a WT before (a) and $\sim 2$ hours after (b) V1Ax $(20 \mu \mathrm{g} / \mathrm{kg}$ ). In (c), shown are example MAP (mmHg, top) and HR (beats/min, bottom) in a WT rat from Group 3 showing the responses (at black vertical dashed lines) to AVP (20ng/kg), showing that the blockade of V1A receptors with Manning Compound was incomplete (see black arrows). 


\section{CHAPTER 5: DISCUSSION}

5-HT neurons within the brainstem, particularly those within the midline raphe, have gained particular attention with respect to how the monoamine neurotransmitters contribute to the maintenance of resting ABP. Caudal raphe 5-HT neurons have direct outputs to autonomic regions and sympathetic preganglionic neurons that contribute to maintaining overall sympathetic nerve activity $[4,131,159]$ and dorsal raphe 5-HT neurons project to regions within the forebrain like hypothalamus (e.g. the PVN), CVO's (e.g the SFO), and hippocampus [322, 323]. 5-HT neurons also secrete other neuromodulators like substance $\mathrm{P}$, thyrotropin releasing hormone and even glutamate [150, 151, 157]. In addition to mediating cardiorespiratory control, including upper airway control, autoresuscitation, central chemoreceptor responses to hypercapnia, and the control of ABP [131, 143], 5-HT and 5-HT neurons are also implicated in the control of the sleep/wake cycle $[4,5,131]$ and the plasticity that follows IH [227]. However, few studies have focused on the role of central 5-HT or 5-HT neurons in the control of ABP or the cardiorespiratory responses to $\mathrm{IH}$ in neonatal rodents or the control of ABP during sleep across development. This dissertation therefore examined the role of CNS 5-HT in the control of ABP, in both neonatal and adult rats. We studied animals in normal conditions (wakefulness and sleep) and, in neonatal animals, in response to IH. In the following sections, my aim is to summarize and discuss the work done in this dissertation as well as provide potential mechanisms mediating the ABP responses in central 5-HT deficiency during the neonatal and adult periods. 


\section{1 - The role of central 5-HT on the control of ABP during sleep in neonatal rodents}

We sought to address whether a modest loss of 5-HT neurons had implications in the control of ABP, as our goal is to better understand the role of 5-HT system dysfunction in the pathophysiology of SIDS. Previous recordings from SIDS cases indicate that infants die following a severe bradycardia and presumably low blood pressure. A vast majority of SIDS cases have one or more 5-HT abnormalities within the brainstem 5-HT system, including a $\sim 30 \%$ reduction in 5-HT content [255]. Therefore, we designed our experiments around the notion that the medullary 5-HT system is partially down-regulated in SIDS. As discussed in Chapter 2 (Aim 1), we found that even a relatively minor depletion of 5-HT neurons alters cardiovascular function in a way that might increase the risk of sudden death for an infant who is confronted with other intrinsic or environmental stressors (i.e. hypoxia). We also found, as described in Chapter 3 (Aim 2), pups specifically lacking central 5-HT have a reduced ABP, but only during QS.

Potential mechanisms contributing to the ABP phenotype in neonatal rodents with central

\section{5-HT deficiency}

The resting $\mathrm{ABP}$ for both treated and control groups were similar to those previously reported for pups of similar age [272, 332], but pups deficient in 5-HT neurons had a reduced sBP, MAP and HR compared to control littermates. To our knowledge, this is the first report indicating a role for 5-HT neurons in resting ABP in rodents at an age roughly equivalent to infancy. In adult animals, central 5-HT signalling contributes to the sympathetic outflow to the cardiac ventricles [273], which may at least partially explain the reduced $\mathrm{ABP}$ phenotype we found in neonatal rodents following a loss of 5-HT neurons 
as only sBP was reduced. Similarly, $T P H 2-/-$ pups also exhibit a reduced ABP compared to WT controls [333]. Thus, it appears as though central 5-HT is mediating the cardiovascular phenotypes in these pups, not the loss of TRH and substance P within the 5-HT neuron [150, 151, 157].

In both TPH2-/- and 5,7-DHT treated pups, the post-synaptic mechanisms leading to reduced $\mathrm{ABP}$ may be similar. For instance, both 5-HT $1 \mathrm{~A}$ and 5-HT2 receptors are known to cause changes in $\mathrm{ABP}$ at various sites within the brainstem. 5- $\mathrm{HT}_{1 \mathrm{~A}}$ activation is typically sympathoinhibitory within regions like the RVLM, the raphe magnus, and raphe pallidus [8], but activation of these receptors within the raphe obscurus using 8-OH-DPAT elicits a pressor response, without affecting HR [334]; this effect in WT pups may help preserve $\mathrm{ABP}$ at this age. To my knowledge, the phenotype of the neurons expressing 5$\mathrm{HT}_{1 \mathrm{~A}}$ receptors in the raphe obscurus is unknown. It is unlikely that this sympathoexcitatory effect of $5-\mathrm{HT}_{1 \mathrm{~A}}$ receptor agonism is through activation of somato-dendritic $5-\mathrm{HT}_{1 \mathrm{~A}}$ receptors, as this would reduce the availability of 5-HT at sites like the RVLM, which contain known excitatory receptors (e.g. 5- $\mathrm{HT}_{2 \mathrm{~A}}$ receptors) [8]. It may be that 5- $\mathrm{HT}_{1 \mathrm{~A}}$ activation acts through a disinhibitory pathway, by binding to $5-\mathrm{HT}_{1 \mathrm{~A}}$ receptors on GABAergic interneurons that project to 5-HT neurons. This would elicit an overall increase in the actions of 5-HT in WT rats, similar to what can occur within cardiac vagal preganglionic neurons [8]. In other words, in rats lacking 5-HT, this disinhibition would be absent, leading to increased GABAergic drive to 5-HT neurons. Indeed, many of the serotonergic nuclei are heterogeneous populations of neurons, containing both serotonergic and non-serotonergic neurons [131], including a population of GABAergic neurons [335]. However, 8-OH-DPAT also activates 5- $\mathrm{HT}_{7}$ receptors, which positively couple to the Gs 
protein, inducing cAMP production [177], confounding these results as 8-OH-DPAT may be preferentially activating $5-\mathrm{HT}_{7}$ receptors within this region. However, to my knowledge, this remains to be tested with specific agonists/antagonists to $5-\mathrm{HT}_{7}$ within the raphe obscurus. Further, the relative contribution of RVLM 5-HT $\mathrm{H}_{1 \mathrm{~A}}$ receptors to ABP in our model may be warranted, as these receptors don't appear to contribute largely to baseline MAP based on previous studies using 5-HT $\mathrm{H}_{1 \mathrm{~A}}$ receptor antagonists [8]. In summary, 5-HT deficiency reduces $\mathrm{ABP}$, possibly through a lack of excitatory neurotransmission at sympathoexcitatory regions like the RVLM.

To begin to assess the mechanism behind the reduced $\mathrm{ABP}$ in TPH2-/- pups, we injected phentolamine (an $\alpha$-adrenergic antagonist) as an indicator of sympathetic drive. We found that TPH2-/- pups had a smaller decrease in ABP to phentolamine [333], suggesting that the vascular component of sympathetic drive is facilitated by central 5-HT. This finding is consistent with pharmacological data obtained from adult cats [288]; vascular resistance is significantly increased in these animals following the application of a 5- $\mathrm{HT}_{2 \mathrm{~A}}$ receptor agonist to the $\mathrm{RVLM}$, without any chronotropic or inotropic effects on the heart [288]. While differences in $\alpha$-adrenergic receptors, in particular $\alpha 1$ adrenoreceptors, in vascular smooth muscle can influence MAP, these effects are likely not influenced by a loss of central 5-HT (although this needs testing). In particular, it would be interesting to assess the number and sensitivity of $\alpha$-adrenergic receptors in vascular smooth muscle to determine their influence on MAP in our model. Further, while local influences from endothelial cells and locally produced chemicals can influence vascular tone, it is more likely that the reduced ABP in TPH2-/- pups compared to WT controls is 
mediated by a central defect, for instance, differences in receptor signaling at the RVLM (i.e. $T P H 2-/$ - pups lack 5-HT mediated 5-HT $\mathrm{H}_{2 \mathrm{~A}}$ signaling) as mentioned briefly above.

As 5-HT neurons exhibit a firing pattern dependent on vigilance-state [223] and project to cholinergic neurons of the LdT/PPT that help initiate and maintain AS/REM sleep [5], we assessed ABP and HR during each of the vigilance-states in TPH2-/- pups. MAP is elevated in QS compared to AS in WT pups, a phenotype that does not exist in TPH2-/- pups. It may be that the increased MAP exhibited by WT pups in QS, relative to AS, is due to increased release of synaptic 5-HT by serotonergic neurons coincident with their increased firing in QS relative to AS; this increased release is an event that does not occur in 5-HT-deficient TPH2-/- animals [223]. Whether the role of 5-HT in the control of $\mathrm{ABP}$ and vigilance-state is through independent pathways or through 5-HT modulation of sleep-related nuclei like the PPT is unknown. As mentioned, 5-HT neurons project to regions involved in the modulation of REM sleep (the LdT/PPT [5]) and to sympathetic premotor regions like the RVLM [284]. However, the PPT also projects to the RVLM [336], lending a potential pathway for sleep-related regions to have a direct impact on cardiovascular control. In our model, the cardiovascular changes associated with the QW to NREM transition were subtle. Therefore, we found it hard to differentiate whether the ABP phenotype during NREM preceded the onset of sleep. However, the cardiovascular changes that occur in REM sleep were easily identifiable -- and occurred after the onset of REM. Therefore, it may be that these cardiovascular changes (i.e. the elevation in ABP) are at least partially mediated by activation of the PPT $\rightarrow$ RVLM pathway.

The vascular component of sympathetic drive is facilitated in males, but not females, by central 5-HT, evidenced by a larger drop in ABP following alpha-adrenoceptor 
blockade in male TPH2-/- pups compared to WT controls. Differences in the levels of sex hormones could be a contributing factor to vascular tone, even during the neonatal period. The sympathoinhibitory role of estrogens has been well-described [291]. However, an interaction between central 5-HT and estrogens in male pups cannot be fully discounted; indeed, there may be slightly reduced plasma estradiol in males compared to females at these ages [291]. Further, testosterone is higher in males compared to females, even at this young age [290], and has known vasodilating properties [289]. Reports suggest that sex steroids like testosterone and estradiol can readily cross the blood-brain barrier [337, 338]; therefore, where the sex steroids are acting (whether peripheral or central) and exactly how sex-specific factors influence the function of central 5-HT in the control of blood pressure during the neonatal period awaits further investigation.

We also found that HR of 5,7-DHT treated and TPH2-/- pups was significantly reduced compared to controls, a finding that has been previously reported in neonatal rodents deficient in 5-HT neurons [158]. Although reduced sympathetic drive to the sinoatrial node could explain this finding, we found that the response to atenolol was not different in male and female TPH2-/- pups compared to controls, suggesting that sympathetic contributions to the heart is not facilitated by central 5-HT at this age. Given that 5-HT neurons send projections to CVNs [274], and that 5-HT activates a number of receptors within these nuclei [61], it is possible that there is increased vagal drive with a loss of 5-HT signalling. Indeed, we found that the rise in HR following atropine was greater in $\mathrm{TPH} 2-/$ - compared to controls suggesting that 5-HT inhibits vagal drive, possibly through activation of 5- $\mathrm{HT}_{1 \mathrm{~A}}$ receptors on glutamatergic inputs to CVNs [168]. However, this phenotype was only seen in female TPH2-/- pups compared to WT controls. Activation 
of G-protein bound estrogen receptors (GPER) depolarizes cardiac vagal neurons in the nucleus ambiguus and microinjection of a GPER agonist into the NA reduces HR [292]. As mentioned, estradiol may be higher in neonatal female rats compared to age matched males [290]; therefore, it may be that in the absence of 5-HT (and 5- $\mathrm{HT}_{1 \mathrm{~A}}$ signalling) that estrogen facilitates cardiac vagal drive. Alternatively, based on anterograde labeling studies, LPGi REM-On neurons also project to the NA, where preganglionic CVNs are located [339]. Indeed, activation of the LPGi with orexin elevates HR, which is at least partially mediated via inhibition of PSNS activity to the heart [86]. Although more direct evidence is required, it may be that 5-HT elicits a similar response as orexin at LPGi REMon neurons, inhibiting PSNS activity of the heart in a vigilance-state dependent fashion. While speculative, it may be that in the absence of 5-HT, LPGi inhibition of PSNS activity may be reduced, lowering HR. Taken together, these results suggest that central 5-HT maintains HR during the neonatal period not by augmenting sympathetic drive but by inhibiting cardiac vagal drive.

The pathways/mechanisms by which central 5-HT controls ABP are likely different from those mediating $\mathrm{HR}$, considering that the ABP of TPH2-/- pups was reduced compared to controls in QS only, while HR was reduced in both QS and AS. Indeed, application of 5- $\mathrm{HT}_{2 \mathrm{~A}}$ receptor agonists to the RVLM increases vascular resistance in cats, without any chronotropic or inotropic effects on the heart [288], suggesting that 5-HT acts through the RVLM to alter ABP but not HR. Further, the reduced HR in neonatal TPH2-/pups appears to be mediated by elevated PSNS tone, further suggesting that central 5-HT reduces parasympathetic, but not sympathetic, drive to the heart. Taken together, the reduced $\mathrm{ABP}$ and $\mathrm{HR}$ in $T P H 2-/-$ rats may be due to: 1 ) reduced 5- $\mathrm{HT}_{1 \mathrm{~A}}$ activation of non- 
serotonergic neurons within the raphe obscurus, causing an altered balance of glutamatergic and GABAergic drive shifted towards GABA; 2) reduced activation of the RVLM resulting from a loss of the activation of excitatory 5- $\mathrm{HT}_{2 \mathrm{~A}}$ receptors; or 3) with respect to $\mathrm{HR}$, increased outflow of CVN's via a disinhibitory pathway. The relative contribution of central $5-\mathrm{HT}_{1 \mathrm{~A}}$ receptors to $\mathrm{ABP}$ in our model may be warranted, as these receptors don't appear to contribute largely to baseline ABP based on previous studies using $5-\mathrm{HT}_{1 \mathrm{~A}}$ receptor antagonists [8].

Factors that influence intrinsic vascular tone (i.e. metabolic or chemical influences) can contribute to basal tone (in the absence of autonomics) and thus MAP. This can include factors like interstitial oxygen levels, bradykinin, and endothelin [340]. However, it is hard to conceptualize how a central defect in 5-HT contributes to altering these factors, and thus basal tone. Although, these factors should not be disregarded. Further, factors contributing to intrinsic HR (HR in the absence of autonomics) is also warranted. We found that intrinsic HR was reduced in neonatal rats lacking central 5-HT and that had a small but significant correlation with reduced body weight. This suggests that central 5-HT contributes to the development of the heart; although functional studies like ultrasonography, on neonatal rodents would make this difficult to directly assess. It may be that altered SNS or PSNS tone to the heart contributes to the development of the heart; we did find that PSNS tone was elevated in female TPH2-/- pups compared to controls but SNS tone was not altered. However, to my knowledge, research has not addressed the extent to which PSNS tone to the heart contributes to its development, including at the level of the SA and AV nodes. This may be interesting to pursue in the future. Further, considering that MAP was reduced in male TPH2-/- pups, possibly due to reduced sympathetic drive (as the responses to 
phentolamine were reduced in these pups compared to WT controls), it would be interesting to evaluate whether reduced SNS tone to the vasculature in neonatal life contributes to remodelling of the vasculature by adulthood. These factors may contribute to the development of hypertension in our model, but remain to be tested.

Acute intermittent hypoxia (AIH) induces an increase in ventilatory and sympathetic motor output that can persist for hours after the end of the stimulus (i.e. ventilatory (vLTF) and sympathetic long-term facilitation (sLTF) [227, 264]). These forms of plasticity are thought to be adaptive, reducing the occurrence of apneas and evidence from adult animals shows that this plasticity is 5-HT dependent $[265,266]$. In neonatal rodents, we also attempted to address 1) whether LTF is present during the neonatal period and 2) whether LTF was 5-HT dependent as seen in adult rodents [227]. Few studies have evaluated how a specific loss of 5-HT affects LTF. Recent work has shown that adult rodents lacking TPH2 have ventilatory LTF, but the magnitude is significantly reduced compared to control littermates [218]. We found the magnitude of vLTF exhibited by two week-old rats was not influenced by a modest loss of 5-HT neurons. Surprisingly, BP rose following AIHH only in pups lacking 5-HT neurons suggesting that during the neonatal period, 5-HT neurons actually constrain the development of sympathetic LTF following AIHH. Recent reports have shown that 5-HT signaling through 5-HT 7 receptors [278] and adenosinergic signaling through $\mathrm{A}_{2 \mathrm{~A}}$ receptors can constrain the development of phrenic LTF [279]. Alternatively, it may be that control pups exhibit a "ceiling" effect, wherein serotonergic pathways that contribute to $\mathrm{ABP}$ are saturated and unamenable to plasticity. Further, whether this is due to a 5-HT neuronal loss in neonates or mediated specifically through the loss of central 5-HT remains to be tested. 


\section{2 - The role of central 5-HT on the control of ABP during sleep in adult rodents}

A significant amount of research has sought to reveal the role of central 5-HT in the control of $\mathrm{ABP}$ and $\mathrm{HR}$ in adult rodents. However, many of these reports are conflicting. For instance, studies utilizing adult rats show that $5-\mathrm{HT}_{3}$ receptor activation within the commissural NTS elicits hypertension [209]. Similarly, cardiac sympathetic nerve activity, $\mathrm{HR}$, and $\mathrm{ABP}$ increase in adult rats treated i.c.v. with DOI, a 5- $\mathrm{HT}_{2 \mathrm{~A}}$ agonist [190]. Furthermore, adult rats systemically treated with para-chlorophenylalanine ( $p$ CPA), a brain permeant 5-HT synthesis inhibitor that depletes CNS and peripheral 5-HT content, had a significant rise in baseline ABP [210]. However, some reports suggest the central 5-HT lowers ABP. For instance, 5,7-DHT treatment in adult rodents reduces ABP [341]. Similarly, mice lacking tryptophan hydroxylase 2, the rate limiting enzyme in the formation of central 5-HT, have a reduced MAP during the early evening [224]. Therefore, considering our findings that during the neonatal period central 5-HT facilitates sympathetic vascular tone, thereby increasing ABP [333], and that sex-specific increases in $\mathrm{ABP}$ occur with aging, we sought to address the hypothesis that central 5-HT preserves ABP mostly during QW and NREM when 5-HT neurons are active. However, contrary to our original hypothesis, we found that by $\sim 6$ months of age, rats lacking central 5-HT exhibited an elevated ABP compared to WT rats, a phenotype that was present across all states, but was most obvious during REM sleep. Therefore, we sought to assess the mechanisms associated with this phenotype. Elevated sympathetic drive [299] and/or angiotensin II (Ang II) [29] activity can contribute to neurogenic hypertension; therefore, we hypothesized that central 5-HT reduces sympathetic drive and/or circulating Ang II. However, we found that $\sim 6$-month-old rats lacking central 5-HT did not exhibit altered 
sympathetic drive nor Ang II signaling compared to controls, suggesting that other mechanisms contribute to the elevated ABP, which I will discuss here.

Potential mechanisms contributing to the ABP phenotype in adult rodents with central 5 HT deficiency

As discussed in Chapter 4 of this dissertation, we found that, contrary to our original hypothesis, adult $\mathrm{TPH} 2-/$ - rodents have an elevated ABP compared to littermate controls, in the absence of differences in sympathetic drive. Considering this phenotype, we hypothesized that a loss of central 5-HT may lead to elevated neurohumoral agents, for instance, Ang II or AVP, stemming from evidence implicating that aberrations in the reninangiotensin system [29] and/or AVP [342] contribute to the development of hypertension. However, evidence within the literature related to the effect of 5-HT on either of these systems is conflicting. For instance, $5-\mathrm{HT}_{2 \mathrm{~A}}$ receptor activation has been shown to activate a central angiotensinergic pathway eliciting the release of AVP, a feature that is required for the development of DOCA-salt hypertension [8]. Furthermore, lesioning of the dorsal raphe inhibits the effect of stress on plasma renin concentration [165]. However, plasma renin activity is elevated following a stressor in rats when the median raphe is lesioned, compared to control rats [166]. Considering the responses to both ganglionic blockade with atropine/hexamethonium and AT1 receptor blockade with losartan were not different in TPH2-/- rats compared to WT controls, this suggests that neither sympathetic drive nor circulating Ang II are mediating the elevated ABP observed in TPH2-/- animals at rest.

Our experiments with a small number of animals suggest the possibility that baroreflex sensitivity (BRS) is reduced in our model of central 5-HT deficiency. Both 
patients with EH [318] and spontaneously hypertensive rats [317] exhibit a reduced BRS. Others have shown that central 5-HT plays a role in BRS; 5-HT depletion with $p$-CPA not only increases ABP, but also reduces BRS [210]. These differences in BRS could be mediated by afferent, central, or efferent components of the baroreflex arc. Furthermore, end organ responsiveness (i.e. at the level of the heart or vasculature) may contribute to altered reflex function or compensate for an impairment in afferent or central defects within the reflex arc [343]. However, whether differences in intrinsic vascular tone are altered in central 5-HT deficiency remain to be elucidated. As previously mentioned, it may be that differences in sympathetic drive during the neonatal period contributes to altered vascular tone, that by adulthood leads to an elevated MAP in TPH2-/- rats; indeed, hypertension is associated with vascular remodeling [344].

Altered BRS occurs in some patients with hypertension [318] and spontaneously hypertensive rats [317]. In our model of 5-HT deficiency, further studies are required to fully elucidate differences in BRS. It appears as though the HR response to phenylephrine is reduced in $T P H 2-/-$ rats, suggesting a reduced BRS. This could reflect defects in the reflex arc; potentially SNS or PSNS drive to the heart (left ventricle, SA, or AV nodes). However, as previously mentioned, there is evidence from sinoaortic denervation (SAD) studies, which shows that SAD results in only a transient increase in MAP and sympathetic nerve activity in animal models, where both MAP and SNA eventually return to near normal levels. This argues against a role for altered BRS in the long term control of MAP [319]. Further, there is some evidence in patients with essential hypertension that sensitivity of baroreflex control of sympathetic vasomotor tone is not attenuated, but is reset to higher levels of arterial pressure [345]. Therefore, at least in older TPH2-/- rats, it 
may be that the baroreflex has reset and other mechanisms are contributing to the maintenance of an elevated MAP in central 5-HT deficiency. Potential avenues to pursue include altered neurohumoral drive, especially vasopressin, which is thought to contribute to the development of hypertension [342]. In summary, as the BRS is reduced in some cases of hypertension [318], including in the rats treated with $p$-CPA [210]; therefore, assessing the extent that central 5-HT contributes to BRS remains to be determined.

In our model, female $T P H 2-/$ rats appear to be protected from the elevated ABP seen in male TPH2-/- rats compared to WT controls. Sex differences in the onset of hypertension have been well established, with a reduced incidence of hypertension in premenopausal women than age-matched men, with an increasing incidence in female over 55 [291]. Ovarian hormones and 5-HT may interact. Evidence suggests that 5-HT neurons not only contain estrogen receptors, but ovarian hormones have been shown to increase TPH expression in rhesus monkeys [324-326]. Further, 5-HT and estrogen receptors can be found in several of the same brain regions within the hypothalamus and brainstem key to the regulation of SNA, HR and BP, including the PVN and RVLM $[4,131,159,320$, 322, 323]. For instance, within the RVLM, activation of ER $\alpha$ receptors with estrogen decreases SNA [321]. Similarly, evidence suggests 5-HT activation of 5- $\mathrm{HT}_{1 \mathrm{~A}}$ mediates a reduction in ABP [346], a phenotype absent in $\mathrm{TPH} 2-/$ - rats due to the loss of central 5HT. Thus, it may be that in female TPH2-/- rats, activation of ER $\alpha$ receptors decreases $\mathrm{ABP}$, compensating for a loss of the inhibitory $5-\mathrm{TH}_{1 \mathrm{~A}}$ mediated signaling.

Higher cognitive areas, like the hippocampus, are mainly thought to mediate behavioral responses, but may also play a role in the modulation of autonomic responses to stress. Indeed, electrical or chemical stimulation of the dorsal or ventral hippocampus 
elicits reductions in both ABP and HR in unanesthetized rats and inactivation of the dorsal hippocampus blocks the cardiovascular response to aversive stimuli. These results implicate the hippocampus in modulating cardiovascular responses to stressful situations [347]. 5-HT neurons synapse at both the hypothalamus [348] and hippocampus [323] and may help mediate the cardiovascular responses to stress. Taken together, if 5-HT activates the hippocampus, i.e. through the 5- $\mathrm{HT}_{2 \mathrm{~A}}$ receptor, it may help mediate the reduced ABP and HR seen in the electrical/chemical stimulations mentioned.

As mentioned, lesioning of the dorsal raphe inhibits the effect of stress on plasma renin concentration [165] but plasma renin activity is elevated following stress in animals with lesioning of the median raphe compared to control rats [166]. Stress is a risk factor in the development of cardiovascular diseases. This occurs through stress-induced activation of the neuroendocrine HPA axis, mediating the release of adrenocorticotropin hormone (ACTH) and glucocorticoids (e.g. cortisol) [349]. Hyperactivity in the HPA axis occurs in the etiology of depression [350], which is associated with 5-HT abnormalities [178]. Further, many depressed patients also manifest hypertension and both depressed and hypertensive patients experience increased ACTH and cortisol secretion [351]. The "stress" involved in the activation of the HPA axis could range from a variety of sources, including increased sensitivity to known "social" stressors like social defeat, isolation [352] (i.e. when the rats are singly housed post catheter surgeries), light-cycle, sound, and cage changing [353], to name a few. Indeed, neonatal maternal separation, a commonly used mechanism of neonatal stress to elucidate the mechanisms associated with fetal programming, leads to elevated ABP in adulthood, but only in male rats. Further, only male rats had elevated ACTH and corticosterone levels when subjected to neonatal maternal 
separation, suggesting that disruption of the HPA axis may mediate the ABP phenotype [354].

Several reports implicate the HPA axis in cardiovascular homeostasis $[355,356]$, including the role of excess glucocorticoids in the development of hypertension [357]. The expression of glucocorticoid (GR) and mineralocorticoid (MR) receptors play a role in controlling the level of HPA output [358], by providing negative feedback when glucocorticoids are elevated by stress. This helps maintain circulating cortisol at low levels and protects against prolonged activity of the HPA axis. Glucocorticoids and 5-HT may interact to play a role in the etiology of hypertension and other physiological HPA outputs, like depression, that can exhibit elevated HPA activity. For instance, 5-HT can increase GR expression [359], including at the level of the hippocampus [360], helping dampen the effects of glucocorticoids on ABP by providing increased negative feedback on the HPA axis during stress. This phenotype is potentially reduced or absent in adult $T P H 2-/-$ rats. Further, 5- $\mathrm{HT}_{1 \mathrm{~A}}$ activation reduces the activity of median eminence projecting PVN neurons, accompanied by sustained decreases in MAP suggesting an inhibitory role for 5HT on HPA activity [361]. Studies inducing a loss of 5-HT further implicate a role for 5HT in this process. Stress normally increases GR expression but this is abolished following 5-HT depletion with p-CPA [362] and central 5-HT lesions following 5,7-DHT treatment [363]. These effects on GR expression may induce an elevated HPA axis activity by blunting the auto-inhibitory effects glucocorticoids normally have on the HPA axis. Thus, a loss of central 5-HT may increase HPA activity through either a loss of auto-inhibitory mechanisms related to the loss of GR and MR receptors or through the loss of 5- $\mathrm{HT}_{1 \mathrm{~A}}$ mediated inhibition of the PVN. However, these hypotheses require further testing. 


\section{3 - The role of central 5-HT in the control of ABP across development}

As we have shown, a loss of 5-HT neurons via treatment with 5,7-DHT leads to a reduced ABP in neonatal rodents [156]. These results suggest that 5-HT signaling prevents ABP from falling. However, as 5-HT neurons also express many other neurotransmitters, including substance P, TRH, enkephalin, and GABA [150, 151], we sought to address whether this phenotype was specifically mediated by central 5-HT. We hypothesized, based on our work in neonatal rodents [364] and work of Alenina and colleagues [224], that central 5-HT indeed prevents ABP from falling. Our hypothesis was only partially confirmed, in that neonatal rodents lacking central 5-HT have a reduced ABP [364] but adult rodents have an increased ABP, compared to WT controls. Thus, it appears as though central 5-HT may play a different role in the control of ABP, depending on age and mode of central 5-HT loss (neuron vs specific loss) or the length of time central 5-HT was depleted (short- vs. long-term). There are many reports individually supporting the findings from Chapters 2-4, as described above for neonatal and adult rodents, respectively. Here I describe three main hypotheses in an attempt to address how central 5-HT may participate in the control of ABP across development.

My first hypothesis is that the reduced ABP observed during the neonatal period in TPH2-/- pups induces a compensatory mechanism (that goes unabated) to counteract the low ABP. Therefore, the adult TPH2-/- have a higher BP than normal due to excessive compensation that has not been resolved to maintain normal ABP. Many reports suggest that fetal stressors during critical stages of development (i.e. during the first two postnatal weeks) induces compensatory mechanisms that lead to an elevated ABP during adulthood [308]. As mentioned previously, the "stress" involved in the activation of the HPA axis 
could range from a variety of sources, including increased sensitivity in $\mathrm{TPH}-\%$ - rats to known "social" stressors like social defeat, isolation [352], light-cycle, sound, and cage changing [353], to name a few. In fact, it may be that the loss of central 5-HT or the reduced ABP in neonatal rodents lacking central 5-HT are the stress itself. Long-term compensations may occur within the respiratory network to mitigate the effects of central 5-HT loss, in that neonatal rodents lacking either serotonin neurons [213] or a specific loss of central 5-HT $[155,214]$ exhibit respiratory dysfunction that is normalized by adulthood. The mechanisms mediating these compensatory changes is speculative at this point, but could stem from alternative sources of excitatory drive when 5-HT is lost (e.g. elevated catecholaminergic drive). Both dopaminergic [365] and noradrenergic [366] systems contribute to cardiovascular regulation. In 5-HT deficiency, these systems may compensate for the loss of 5-HT. Even though this hypothesis is currently speculative, there is evidence that lesioning the dopaminergic system during the neonatal period increases sprouting and proliferation of the 5-HT system, which arise primarily from dorsal raphe afferents [367]. Further, striatal lesions of catecholaminergic neurons with 6-OHDA increases the spontaneous firing rates of DRN 5-HT neurons [368]. These studies suggest that compensation within other neurotransmitter systems can occur to counteract the loss of excitatory neurotransmission. Whether this occurs in cardiovascular sites is unknown. However, considering that not only phrenic but sympathetic plasticity occurs following intermittent episodes of hypoxia [227], this suggests that compensatory mechanisms may occur in both central respiratory and cardiovascular regions.

My second hypothesis is that the rise in ABP across development may be mediated through the HPA axis. As mentioned, hyperactivity in the HPA axis occurs in the etiology 
of depression in adult rodents [350], which is commonly associated with 5-HT system abnormalities [178], and both depressive and hypertensive patients experience increased ACTH and cortisol secretion [351]. Indeed, neonatal maternal separation leads to elevated ABP in adulthood, but only in male rats, possibly mediated by a disrupted HPA axis function as both plasma ACTH and corticosterone levels were elevated in males but not females [354]. Unfortunately, this study did not assess ABP during the neonatal period. However, neonates experience what is called a "stress hypo-responsive period (SHRP)", where they experience a reduced capacity for secretion of ACTH and corticosterone in response to several types of mild stress. Despite this SHRP, neonates do retain an adultlike HPA axis response to elevated corticosterone levels in life-threatening situations, e.g. during severe hypoxia. Corticosteroids during the neonatal period can affect a variety of processes, including inhibition of neuronal cell division, glial development and retardation of brain growth. Thus, the SHRP is thought of as a protective mechanism against the fatal impact of circulating corticosteroids on immature neurons. During the SHRP, corticosterone remains low until after the second postnatal week, then starts to rise [369]. In summary, it is possible that in light of a reduced SHRP, the overall effects of central 5HT deficiency are unmasked by any potential interactions with corticosterone, and central 5-HT may indeed increase ABP. Alternatively, the "hypotensive" or "5-HT deficient" phenotypes may be what is considered the "stress" during this critical period of development that ultimately leads to an elevated HPA axis phenotype in adulthood. However, these hypotheses remain to be tested.

During the neonatal period, central 5-HT deficiency may be a "stressor" that leads to long-term consequences in GR expression or functionality during the neonatal period or 
across development, that ultimately leads to a hypertensive phenotype in adults. Evidence suggests that there is a gradual increase in GR mRNA expression from birth, which by P16, reaches adult levels in other models. Whether this increase in GR mRNA is affected by the SHRP or 5-HT deficiency is unknown. Further, not only corticosteroid levels, but also GR expression and GR functionality, are altered in emotional disorders including depression (i.e. by adulthood). Whether corticosteroid ligand-receptor changes are a cause or consequence in disorders like depression are unknown - and difficult to prove in rodent models. However, early-life stress can lead to long-term differences in the expression and functionality of the GR relative to control groups [370]. Considering that many depressed patients also manifest hypertension [351], it may be that the altered GR expression in depression, following early life stress, contributes to the hypertensive phenotype. As mentioned, 5-HT can increase GR expression [359], which may help dampen the effects of HPA activity on ABP by increasing the negative feedback provided by glucocorticoids on GR's. This phenotype is potentially reduced or absent in TPH2-/- rats. In summary, 5HT deficiency may invoke an early life stress that causes long-term differences in GR expression/functionality that by adulthood leads to a hypertensive phenotype.

My third hypothesis is that differences in 5-HT receptor expression, sensitivity, or the turnover of 5-HT receptors across development may contribute to the ABP in WT controls, a phenotype that would be absent in $T P H 2-/$ - rats. Reports have suggested that neonatal rodents lack responsiveness to $5 \mathrm{HT}_{1 \mathrm{~A}}$ autoreceptor activation, suggesting that 5HT neurons have an increased excitability and 5-HT release during the neonatal period [371]. Further, some have reported that 5-HT levels are the highest at P14 before falling to adult levels by approximately 3 weeks of age [372], making the difference in 5-HT content 
between a $\mathrm{TPH} 2-/$ and WT counterparts greatest during this period. The mechanism behind hyper-excitable 5-HT neurons during the neonatal period appears mediated through the 5-HT $\mathrm{T}_{1 \mathrm{~A}}$ effector system (e.g. G-protein), which is absent and non-functional at $\mathrm{P} 4$ but present and functional by adulthood [371]. The hyper-excitable 5-HT neuron during the neonatal period may affect the level of not only 5-HT release, but also the release of substance P and TRH. In WT pups, this could mean an elevated release of excitatory neurotransmitters that includes 5-HT, which may be particularly important for ABP regulation during the neonatal period. However, assessing 5-HT, and for that matter, Substance P and TRH, release from 5-HT neurons near cardiovascular regions across development may help determine whether differences in these neurotransmitters between WT and $T P H 2-/-$ contributes to the MAP phenotype in neonates vs. adult rodents is warranted.

By adulthood, 5- $\mathrm{HT}_{1 \mathrm{~A}}$ autoreceptor responsiveness is present, contrary to the neonatal period [371]. It may be that the level of neurotransmitter release from 5-HT neurons in a WT rat is altered during development, while the responsiveness of the 5- $\mathrm{HT}_{1 \mathrm{~A}}$ autoreceptor becomes "adult-like". The lack of 5-HT in $\mathrm{TPH} 2-/$ - rats reduces the possibility of 5- $\mathrm{HT}_{1 \mathrm{~A}}$ mediated autoinhibition of 5-HT neurons. Therefore, it may be that $T P H 2-/$ - rats have an elevated release (compared to WT counterparts) of co-expressed neuromodulators like substance P and TRH $[150,151]$, which have well known roles in cardiovascular homeostasis [296, 297]. However, electrophysiological testing of 5-HT neuronal properties in the $\mathrm{TPH} 2-/$ - rat may be warranted, to verify whether the pacemaker properties of 5-HT neurons in the absence of endogenous 5-HT is altered. Indeed, in TPH2-/- mice, there is a slightly but significantly reduced firing rate compared to WT controls, which may indicate 
that 5-HT neurons adapt their membrane properties to compensate for the loss of autoinhibition and to keep pacemaker firing activity constant [373].

In summary, the role of central 5-HT in the control of ABP flips between neonatal and adult rodents and the mechanisms associated with this switch are unknown. The phenotype may be associated with compensatory mechanisms to increase the low ABP in TPH2-/- pups, fetal programming in TPH2-/- pups, or changes in receptor profiles across development (whether GR or the 5- $\mathrm{HT}_{1 \mathrm{~A}}$ autoreceptor) that ultimately lead to an elevated ABP in adulthood. Whether these occur requires future studies.

\section{$\underline{5.4-\text { Summary and Potential Future Studies }}$}

Taken together, these three studies support an important role for central 5-HT in the maintenance of ABP across development. Moving forward, continuing to address the mechanism(s) behind the reduced ABP and bradycardia in neonatal rats and the elevated ABP in adult male rats following chronic serotonin depletion is warranted. Pups lacking central 5-HT had a smaller decrease in ABP in response to phentolamine suggesting that the vascular component of sympathetic drive is facilitated by central 5-HT. While differences in $\alpha$-adrenergic receptors, in particular $\alpha 1$-adrenoreceptors, in vascular smooth muscle can influence ABP, these effects are likely not influenced by a loss of central 5HT. However, differences in the number or sensitivity of vascular $\alpha 1$-adrenoreceptors needs testing. Further, as 5-HT deficiency reduces ABP, it is possible that a lack of excitatory neurotransmission at sympathoexcitatory regions like the RVLM, explain this phenotype. For instance, differences in receptor signaling at the RVLM (i.e. TPH2-/- pups lack 5-HT mediated 5-HT2A signaling). Addressing whether this occurs as a direct pathway, 
through 5-HT projections to the RVLM or indirectly through sleep related regions (e.g. through 5-HT projections to the PPT, which projects to the RVLM), would be worth testing. Further, considering the bradycardia in female, but not male, TPH2-/- pups was mediated by an elevated cardiac vagal tone, it may be worthwhile to explore the mechanism and the extent that estrogen and estrogen receptors contribute to this phenotype.

In adults, we have shown that the elevated ABP occurs without elevated sympathetic activity, as evidenced by a lack of genotype effect in the fall in ABP to ganglionic blockade. Considering ganglionic blockade is not a direct measurement of sympathetic activity nor does it allow us to differentiate between differences in sympathetic nerve activity versus intrinsic vascular tone, it may be worthwhile to measure sympathetic nerve activity in our model. Further, while peripheral 5-HT is a known vasoconstrictor [199], a role for central 5-HT in intrinsic vascular tone has yet to be assessed. Vascular changes in hypertension are common; outward hypertrophic remodeling and increased stiffness within large arteries occurs with aging and hypertension is thought to accelerate this process [344]. However, if this occurs in our model, whether it is a function of the hypertension or the loss of central 5-HT would require testing. Further, cardiac remodeling occurs with aging and hypertension is thought to contribute to left ventricular hypertrophy [374]. However, whether cardiac remodeling occurs in our model and whether it is a function of the hypertension or the loss of central 5-HT signaling requires testing.

Similarly, considering evidence that reduced baroreflex sensitivity occurs in hypertension [317, 318], and that central 5-HT appears to play a role in BRS [210], assessing BRS in our model is warranted. As female TPH2-/- rats appear to be protected from the elevated $\mathrm{ABP}$ phenotype, it may be that BRS sensitivity in female rats is greater 
than in male rats as a protective mechanism - in addition to the potential protective effects of estrogens [375]. Indeed, BRS is higher in female rats during the mid-luteal phase, when estrogen levels are elevated [376] and estrogen increases BRS in ovariectomized female rats [377]. Considering evidence that suggests central 5-HT and ovarian hormones may interact [324-326], it may be that estrogens preserve the increased BRS and help preserve ABP, even in the face of 5-HT deficiency. However, as we originally did not anticipate a sex effect in the control of ABP in the face of 5-HT deficiency, we did not account for phase of the estrous cycle in our study. Therefore, experiments to not only assess if the phenotype is phase dependent and whether ovariectomy reverses the phenotype, whether BRS contributes to the protective phenotype in females in 5-HT deficiency remains to be elucidated.

While BRS may be reduced in adult rodents lacking central 5-HT, the elevated ABP in central 5-HT deficiency may be due to an overactive HPA axis, as discussed (also, see Fig. 5.1 as a schematic showing the potential pathway involved in HPA activity). Assessing circulating corticosterone levels or the response to GR antagonism may shed light on this possibility. We hypothesize that central 5-HT inhibits the HPA axis and reduces circulating corticosterone levels to help prevent a rise in ABP. Further, addressing the possibility that fetal programming occurs during the neonatal period and leads to the elevated HPA axis may be warranted.

In conclusion, the present study demonstrated a key role for central 5-HT in the control of $\mathrm{ABP}$ in both neonatal and adult rodents during sleep. Future studies will be needed to provide further insight into the mechanisms by which central 5-HT influences 
$\mathrm{ABP}$ in the neonatal period compared to during adulthood and how sex specific factors influence the function of central 5-HT in the control of ABP. 


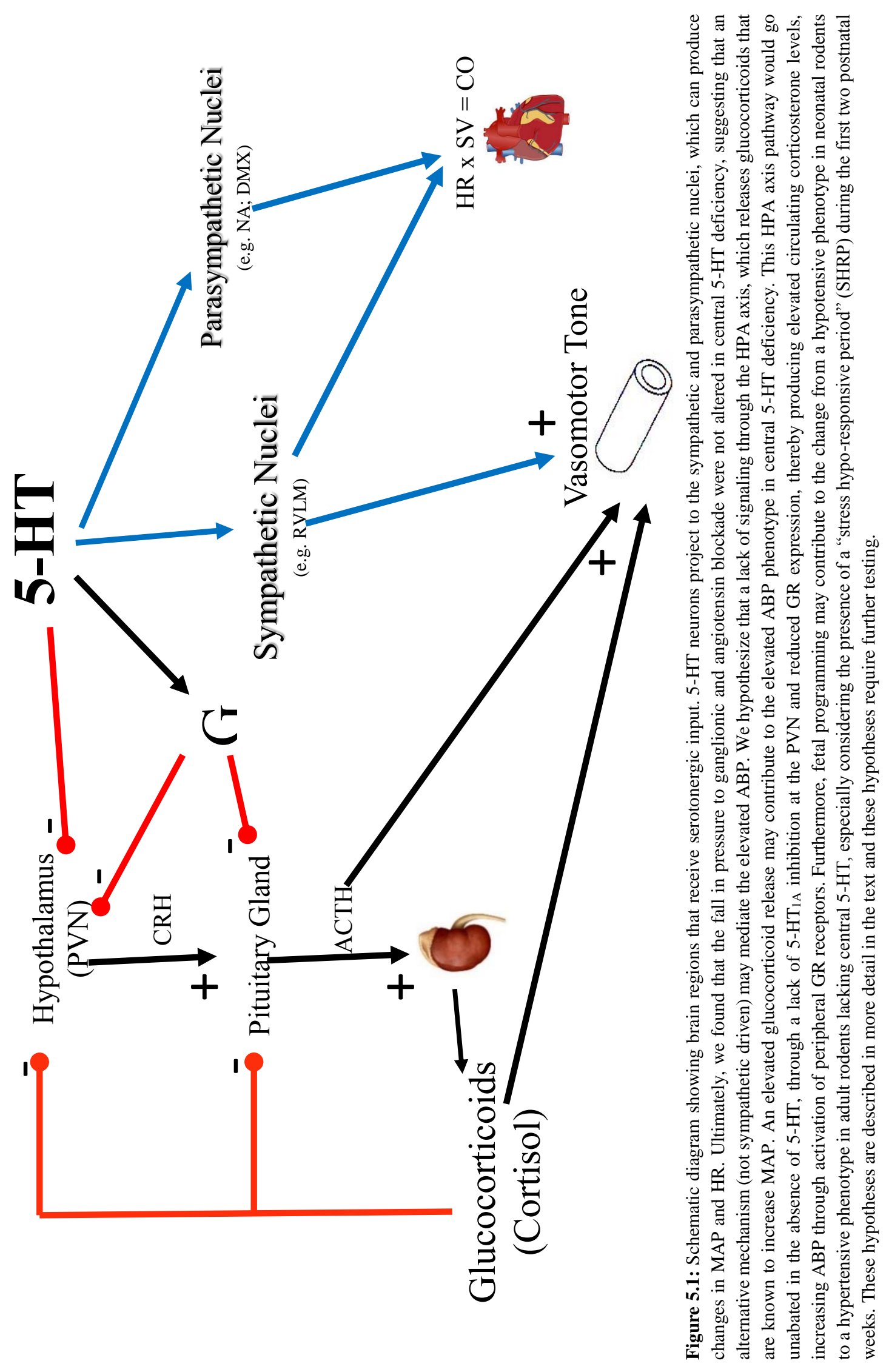




\section{LIST OF REFERENCES}

1. Mohammad-Zadeh, L.F., L. Moses, and S.M. Gwaltney-Brant, Serotonin: a review. J Vet Pharmacol Ther, 2008. 31(3): p. 187-99.

2. Hasan, W., Autonomic cardiac innervation: development and adult plasticity. Organogenesis, 2013. 9(3): p. 176-93.

3. Saper, C.B., T.C. Chou, and T.E. Scammell, The sleep switch: hypothalamic control of sleep and wakefulness. Trends Neurosci, 2001. 24(12): p. 726-31.

4. Benarroch, E.E., Medullary serotonergic system: organization, effects, and clinical correlations. Neurology, 2014. 83(12): p. 1104-11.

5. $\quad$ Brown, R.E., et al., Control of sleep and wakefulness. Physiol Rev, 2012. 92(3): p. 1087187.

6. $\quad$ Saper, C.B., et al., Sleep state switching. Neuron, 2010. 68(6): p. 1023-42.

7. Marshall, J.M., Peripheral chemoreceptors and cardiovascular regulation. Physiol Rev, 1994. 74(3): p. 543-94.

8. Ramage, A.G. and C.M. Villalon, 5-hydroxytryptamine and cardiovascular regulation. Trends Pharmacol Sci, 2008. 29(9): p. 472-81.

9. Watts, S.W., 5-HT in systemic hypertension: foe, friend or fantasy? Clin Sci (Lond), 2005. 108(5): p. 399-412.

10. Charnay, Y. and L. Leger, Brain serotonergic circuitries. Dialogues in clinical neuroscience, 2010. 12(4): p. 471-87.

11. Guyenet, P.G., The sympathetic control of blood pressure. Nat Rev Neurosci, 2006. 7(5): p. 335-46.

12. Charnay, Y. and L. Leger, Brain serotonergic circuitries. Dialogues Clin Neurosci, 2010. 12(4): p. 471-87.

13. Routal, R.V. and G.P. Pal, Location of the phrenic nucleus in the human spinal cord. J Anat, 1999. 195 ( Pt 4): p. 617-21.

14. Webber, C.L., Jr., R.D. Wurster, and J.M. Chung, Cat phrenic nucleus architecture as revealed by horseradish peroxidase mapping. Experimental brain research, 1979. 35(3): p. 395-406.

15. DeVries, K.L. and H.G. Goshgarian, Spinal cord localization and characterization of the neurons which give rise to the accessory phrenic nerve in the adult rat. Experimental neurology, 1989. 104(1): p. 88-90.

16. West, J.B., Respiratory physiology : the essentials. 9th ed. 2012, Philadelphia: Wolters Kluwer Health/Lippincott Williams \& Wilkins. viii, 200 pages.

17. Katz, A.M., Physiology of the heart. 2nd ed. 1992, New York: Raven Press. xvi, 687 pages.

18. Stiles, G.L. and R.J. Lefkowitz, Cardiac adrenergic receptors. Annu Rev Med, 1984. 35 : p. 149-64.

19. Michel, M.C., O.E. Brodde, and P.A. Insel, Peripheral adrenergic receptors in hypertension. Hypertension, 1990. 16(2): p. 107-20.

20. Ram, C.V.S., Beta-blockers in hypertension. The American journal of cardiology, 2010. 106(12): p. 1819-25.

21. Ripley, T.L. and J.J. Saseen, beta-blockers: a review of their pharmacological and physiological diversity in hypertension. The Annals of pharmacotherapy, 2014. 48(6): p. 723-33.

22. Vanhoutte, P.M. and M.N. Levy, Prejunctional cholinergic modulation of adrenergic neurotransmission in the cardiovascular system. Am J Physiol, 1980. 238(3): p. H275-81.

23. Casadei, B., et al., Baroreflex control of stroke volume in man: an effect mediated by the vagus. J Physiol, 1992. 448: p. 539-50.

24. Gordan, R., J.K. Gwathmey, and L.H. Xie, Autonomic and endocrine control of cardiovascular function. World J Cardiol, 2015. 7(4): p. 204-14. 
25. Reid, I.A., Interactions between ANG II, sympathetic nervous system, and baroreceptor reflexes in regulation of blood pressure. The American journal of physiology, 1992. 262(6 Pt 1): p. E763-78.

26. Zimmerman, B.G., S.K. Gomer, and J.C. Liao, Action of angiotensin on vascular adrenergic nerve endings: facilitation of norepinephrine release. Fed Proc, 1972. 31(4): p. 1344-50.

27. Malik, K.U. and A. Nasjletti, Facilitation of adrenergic transmission by locally generated angiotensin II in rat mesenteric arteries. Circ Res, 1976. 38(1): p. 26-30.

28. Ufnal, M. and J. Skrzypecki, Blood borne hormones in a cross-talk between peripheral and brain mechanisms regulating blood pressure, the role of circumventricular organs. Neuropeptides, 2014. 48(2): p. 65-73.

29. Veerasingham, S.J. and M.K. Raizada, Brain renin-angiotensin system dysfunction in hypertension: recent advances and perspectives. Br J Pharmacol, 2003. 139(2): p. 191202.

30. Keller-Wood, M., Hypothalamic-Pituitary--Adrenal Axis-Feedback Control. Compr Physiol, 2015. 5(3): p. 1161-82.

31. Burford, N.G., N.A. Webster, and D. Cruz-Topete, Hypothalamic-Pituitary-Adrenal Axis Modulation of Glucocorticoids in the Cardiovascular System. Int J Mol Sci, 2017. 18(10).

32. Mifflin, S.W., Intensity and frequency dependence of laryngeal afferent inputs to respiratory hypoglossal motoneurons. J Appl Physiol (1985), 1997. 83(6): p. 1890-9.

33. Levitzky, M.G., Pulmonary physiology, in Lange medical book. 2007, McGraw-Hill Medical,: New York. p. 1 electronic text.

34. Feldman, J.L., G.S. Mitchell, and E.E. Nattie, Breathing: rhythmicity, plasticity, chemosensitivity. Annu Rev Neurosci, 2003. 26: p. 239-66.

35. Franchini, K.G. and E.M. Krieger, Cardiovascular responses of conscious rats to carotid body chemoreceptor stimulation by intravenous KCN. J Auton Nerv Syst, 1993. 42(1): p. 63-9.

36. Prabhakar, N.R., Neurotransmitters in the carotid body. Adv Exp Med Biol, 1994. 360: p. 57-69.

37. Kumar, P. and N.R. Prabhakar, Peripheral chemoreceptors: function and plasticity of the carotid body. Compr Physiol, 2012. 2(1): p. 141-219.

38. Kline, D.D., et al., Altered respiratory responses to hypoxia in mutant mice deficient in neuronal nitric oxide synthase. J Physiol, 1998. 511 ( Pt 1): p. 273-87.

39. Kline, D.D., et al., Blunted respiratory responses to hypoxia in mutant mice deficient in nitric oxide synthase-3. J Appl Physiol (1985), 2000. 88(4): p. 1496-508.

40. Peng, Y.J., et al., Complementary roles of gasotransmitters $\mathrm{CO}$ and H2S in sleep apnea. Proc Natl Acad Sci U S A, 2017. 114(6): p. 1413-1418.

41. Talman, W.T., M.H. Perrone, and D.J. Reis, Evidence for L-glutamate as the neurotransmitter of baroreceptor afferent nerve fibers. Science (New York, N Y ), 1980. 209(4458): p. 813-5.

42. Kline, D.D., Plasticity in glutamatergic NTS neurotransmission. Respir Physiol Neurobiol, 2008. 164(1-2): p. 105-11.

43. Andresen, M.C. and D.L. Kunze, Nucleus tractus solitarius--gateway to neural circulatory control. Annu Rev Physiol, 1994. 56: p. 93-116.

44. Andresen, M.C. and D. Mendelowitz, Sensory afferent neurotransmission in caudal nucleus tractus solitarius--common denominators. Chemical senses, 1996. 21(3): p. 38795.

45. Frigero, M., L.G. Bonagamba, and B.H. Machado, The gain of the baroreflex bradycardia is reduced by microinjection of NMDA receptor antagonists into the nucleus tractus solitarii of awake rats. J Auton Nerv Syst, 2000. 79(1): p. 28-33. 
46. Machado, B.H., et al., Neurotransmission of autonomic components of aortic baroreceptor afferents in the NTS of awake rats. Am J Physiol Heart Circ Physiol, 2000. 279(1): p. H6775.

47. Costa-Silva, J.H., D.B. Zoccal, and B.H. Machado, Glutamatergic antagonism in the NTS decreases post-inspiratory drive and changes phrenic and sympathetic coupling during chemoreflex activation. Journal of neurophysiology, 2010. 103(4): p. 2095-106.

48. Costa, K.M., D.J. Moraes, and B.H. Machado, Acute inhibition of glial cells in the NTS does not affect respiratory and sympathetic activities in rats exposed to chronic intermittent hypoxia. Brain Res, 2013. 1496: p. 36-48.

49. Callera, J.C., et al., Activation of GABAA but not GABAB receptors in the NTSblocked bradycardia of chemoreflex in awake rats. Am J Physiol, 1999. 276(6 Pt 2): p. H1902-10.

50. Merahi, N., et al., Baroreceptor reflex inhibition induced by the stimulation of serotonin 3 receptors in the nucleus tractus solitarius of the rat. Neuroscience, 1992. 46(1): p. 91-100.

51. Nosjean, A., B. Franc, and R. Laguzzi, Increased sympathetic nerve discharge without alteration in the sympathetic baroreflex response by serotonin 3 receptor stimulation in the nucleus tractus solitarius of the rat. Neuroscience letters, 1995. 186(1): p. 41-4.

52. Sevoz, C., et al., Stimulation of 5-HT3 receptors in the NTS inhibits the cardiac BezoldJarisch reflex response. Am J Physiol, 1996. 271(1 Pt 2): p. H80-7.

53. Damaso, E.L., et al., Involvement of central 5-HT7 receptors in modulation of cardiovascular reflexes in awake rats. Brain research, 2007. 1144: p. 82-90.

54. Sved, A.F., S. Ito, and C.J. Madden, Baroreflex dependent and independent roles of the caudal ventrolateral medulla in cardiovascular regulation. Brain Res Bull, 2000. 51(2): p. 129-33.

55. Sved, A.F., et al., Excitatory inputs to the RVLM in the context of the baroreceptor reflex. Ann N Y Acad Sci, 2001. 940: p. 247-58.

56. Ross, C.A., et al., Tonic vasomotor control by the rostral ventrolateral medulla: effect of electrical or chemical stimulation of the area containing C1 adrenaline neurons on arterial pressure, heart rate, and plasma catecholamines and vasopressin. J Neurosci, 1984. 4(2): p. 474-94.

57. Dampney, R.A., et al., Central mechanisms underlying short- and long-term regulation of the cardiovascular system. Clin Exp Pharmacol Physiol, 2002. 29(4): p. 261-8.

58. Stornetta, R.L., Neurochemistry of bulbospinal presympathetic neurons of the medulla oblongata. J Chem Neuroanat, 2009. 38(3): p. 222-30.

59. Dubreuil, V., et al., A human mutation in Phox $2 b$ causes lack of CO2 chemosensitivity, fatal central apnea, and specific loss of parafacial neurons. Proc Natl Acad Sci U S A, 2008. 105(3): p. 1067-72.

60. Kanbar, R., et al., Photostimulation of Phox $2 b$ medullary neurons activates cardiorespiratory function in conscious rats. Am J Respir Crit Care Med, 2010. 182(9): p. 1184-94.

61. Jordan, D., Vagal control of the heart: central serotonergic (5-HT) mechanisms. Exp Physiol, 2005. 90(2): p. 175-81.

62. Taylor, E.W., D. Jordan, and J.H. Coote, Central control of the cardiovascular and respiratory systems and their interactions in vertebrates. Physiological reviews, 1999. 79(3): p. 855-916.

63. Damasceno, R.S., A.C. Takakura, and T.S. Moreira, Regulation of the chemosensory control of breathing by Kolliker-Fuse neurons. Am J Physiol Regul Integr Comp Physiol, 2014. 307(1): p. R57-67.

64. Hirooka, Y., et al., Hypoxia-induced Fos expression in neurons projecting to the pressor region in the rostral ventrolateral medulla. Neuroscience, 1997. 80(4): p. 1209-24. 
65. Dutschmann, M. and H. Herbert, The Kolliker-Fuse nucleus gates the postinspiratory phase of the respiratory cycle to control inspiratory off-switch and upper airway resistance in rat. Eur J Neurosci, 2006. 24(4): p. 1071-84.

66. Smith, J.C., et al., Brainstem respiratory networks: building blocks and microcircuits. Trends Neurosci, 2013. 36(3): p. 152-62.

67. Guyenet, P.G., et al., The retrotrapezoid nucleus and breathing. Adv Exp Med Biol, 2012. 758: p. 115-22.

68. Guyenet, P.G. and D.A. Bayliss, Neural Control of Breathing and CO2 Homeostasis. Neuron, 2015. 87(5): p. 946-61.

69. Nattie, E. and A. Li, Central chemoreception is a complex system function that involves multiple brain stem sites. J Appl Physiol (1985), 2009. 106(4): p. 1464-6.

70. Gray, P.A., et al., Developmental origin of preBotzinger complex respiratory neurons. J Neurosci, 2010. 30(44): p. 14883-95.

71. Garcia, A.J., 3rd, et al., Chapter 3--networks within networks: the neuronal control of breathing. Prog Brain Res, 2011. 188: p. 31-50.

72. Johnson, S.M., N. Koshiya, and J.C. Smith, Isolation of the kernel for respiratory rhythm generation in a novel preparation: the pre-Botzinger complex "island". J Neurophysiol, 2001. 85(4): p. 1772-6.

73. Marchenko, V., et al., Perturbations of Respiratory Rhythm and Pattern by Disrupting Synaptic Inhibition within Pre-Botzinger and Botzinger Complexes. eNeuro, 2016. 3(2).

74. Nattie, E. and A. Li, Central chemoreception 2005: a brief review. Auton Neurosci, 2006. 126-127: p. 332-8.

75. Squire, L.R., Fundamental neuroscience. 3rd ed. 2008, Amsterdam ; Boston: Academic Press/Elsevier. xx, 1,256 pages.

76. Smith, J.C., et al., Structural and functional architecture of respiratory networks in the mammalian brainstem. Philos Trans R Soc Lond B Biol Sci, 2009. 364(1529): p. 2577-87.

77. Sun, Q.J., et al., Botzinger neurons project towards bulbospinal neurons in the rostral ventrolateral medulla of the rat. The Journal of comparative neurology, 1997. 388(1): $\mathrm{p}$. 23-31.

78. Fedorko, L. and E.G. Merrill, Axonal projections from the rostral expiratory neurones of the Botzinger complex to medulla and spinal cord in the cat. J Physiol, 1984. 350: p. 48796.

79. Datta, S. and R.R. Maclean, Neurobiological mechanisms for the regulation of mammalian sleep-wake behavior: reinterpretation of historical evidence and inclusion of contemporary cellular and molecular evidence. Neurosci Biobehav Rev, 2007. 31(5): p. 775-824.

80. Murillo-Rodriguez, E., et al., Mechanisms of sleep-wake cycle modulation. CNS Neurol Disord Drug Targets, 2009. 8(4): p. 245-53.

81. Smale, L., T. Lee, and A.A. Nunez, Mammalian diurnality: some facts and gaps. Journal of biological rhythms, 2003. 18(5): p. 356-66.

82. Mistlberger, R.E., Circadian regulation of sleep in mammals: role of the suprachiasmatic nucleus. Brain Res Brain Res Rev, 2005. 49(3): p. 429-54.

83. Jones, B.E., Arousal systems. Front Biosci, 2003. 8: p. s438-51.

84. Merica, H. and R.D. Fortune, State transitions between wake and sleep, and within the ultradian cycle, with focus on the link to neuronal activity. Sleep Med Rev, 2004. 8(6): p. 473-85.

85. Swick, T.J., The neurology of sleep. Neurol Clin, 2005. 23(4): p. 967-89, v.

86. Sirieix, C., et al., Role of the lateral paragigantocellular nucleus in the network of paradoxical (REM) sleep: an electrophysiological and anatomical study in the rat. PLoS One, 2012. 7(1): p. e28724. 
87. Toscani, L., et al., Human heart rate variability and sleep stages. Ital J Neurol Sci, 1996. 17(6): p. 437-9.

88. Verrier, R.L., et al., Primary vagally mediated decelerations in heart rate during tonic rapid eye movement sleep in cats. Am J Physiol, 1998. 274(4 Pt 2): p. R1136-41.

89. Snyder, F., et al., Changes in Respiration, Heart Rate, and Systolic Blood Pressure in Human Sleep. J Appl Physiol, 1964. 19: p. 417-22.

90. Buchanan, G.F., Timing, sleep, and respiration in health and disease. Progress in molecular biology and translational science, 2013. 119: p. 191-219.

91. Sowho, M., et al., Sleep and respiratory physiology in adults. Clin Chest Med, 2014. 35(3): p. 469-81.

92. Buchanan, G.F., Timing, sleep, and respiration in health and disease. Prog Mol Biol Transl Sci, 2013. 119: p. 191-219.

93. Coote, J.H., Respiratory and circulatory control during sleep. J Exp Biol, 1982. 100: p. 223-44.

94. Elsenbruch, S., M.J. Harnish, and W.C. Orr, Heart rate variability during waking and sleep in healthy males and females. Sleep, 1999. 22(8): p. 1067-71.

95. Ayas, N.T., R. Brown, and S.A. Shea, Hypercapnia can induce arousal from sleep in the absence of altered respiratory mechanoreception. Am J Respir Crit Care Med, 2000. 162(3 Pt 1): p. 1004-8.

96. Li, A. and E. Nattie, Orexin, cardio-respiratory function, and hypertension. Front Neurosci, 2014. 8: p. 22.

97. Guyenet, P.G. and S.B. Abbott, Chemoreception and asphyxia-induced arousal. Respir Physiol Neurobiol, 2013. 188(3): p. 333-43.

98. Bowes, G., et al., Effect of carotid body denervation on arousal response to hypoxia in sleeping dogs. J Appl Physiol Respir Environ Exerc Physiol, 1981. 51(1): p. 40-5.

99. Bowes, G., et al., Role of the carotid body and of afferent vagal stimuli in the arousal response to airway occlusion in sleeping dogs. Am Rev Respir Dis, 1981. 123(6): p. 6447.

100. Abbott, S.B., et al., Optogenetic stimulation of $c 1$ and retrotrapezoid nucleus neurons causes sleep state-dependent cardiorespiratory stimulation and arousal in rats. Hypertension, 2013. 61(4): p. 835-41.

101. Horner, R.L., et al., Effects of sleep-wake state on the genioglossus vs.diaphragm muscle response to CO(2) in rats. J Appl Physiol (1985), 2002. 92(2): p. 878-87.

102. Berry, R.B. and K. Gleeson, Respiratory arousal from sleep: mechanisms and significance. Sleep, 1997. 20(8): p. 654-75.

103. Pyner, S. and J.H. Coote, Identification of branching paraventricular neurons of the hypothalamus that project to the rostroventrolateral medulla and spinal cord. Neuroscience, 2000. 100(3): p. 549-56.

104. Silvani, A. and R.A. Dampney, Central control of cardiovascular function during sleep. Am J Physiol Heart Circ Physiol, 2013. 305(12): p. H1683-92.

105. Alam, M.N., et al., Adenosinergic modulation of rat basal forebrain neurons during sleep and waking: neuronal recording with microdialysis. J Physiol, 1999. 521 Pt 3: p. 679-90.

106. Haas, H. and P. Panula, The role of histamine and the tuberomamillary nucleus in the nervous system. Nat Rev Neurosci, 2003. 4(2): p. 121-30.

107. Thakkar, M.M., Histamine in the regulation of wakefulness. Sleep Med Rev, 2011. 15(1): p. 65-74.

108. Johnson, P.L., et al., Are tuberomammillary histaminergic neurons involved in CO2mediated arousal? Exp Neurol, 2005. 193(1): p. 228-33.

109. Sherin, J.E., et al., Innervation of histaminergic tuberomammillary neurons by GABAergic and galaninergic neurons in the ventrolateral preoptic nucleus of the rat. J Neurosci, 1998. 18(12): p. 4705-21. 
110. Haxhiu, M.A., et al., Monoaminergic neurons, chemosensation and arousal. Respir Physiol, 2001. 129(1-2): p. 191-209.

111. Dutschmann, M., et al., Histaminergic modulation of the intact respiratory network of adult mice. Pflugers Arch, 2003. 445(5): p. 570-6.

112. Bhuiyan, M.E., et al., Histamine receptor $\mathrm{Hl}$ in the nucleus tractus solitarii regulates arterial pressure and heart rate in rats. Am J Physiol Heart Circ Physiol, 2011. 301(2): p. H523-9.

113. Ishiguro, T., et al., Impaired ventilation and metabolism response to hypoxia in histamine H1 receptor-knockout mice. Respir Physiol Neurobiol, 2006. 154(3): p. 331-41.

114. Biancardi, V., et al., Locus coeruleus noradrenergic neurons and CO2 drive to breathing. Pflugers Arch, 2008. 455(6): p. 1119-28.

115. Carter, M.E., et al., Tuning arousal with optogenetic modulation of locus coeruleus neurons. Nat Neurosci, 2010. 13(12): p. 1526-33.

116. Carter, M.E., et al., Mechanism for Hypocretin-mediated sleep-to-wake transitions. Proc Natl Acad Sci U S A, 2012. 109(39): p. E2635-44.

117. Gargaglioni, L.H., L.K. Hartzler, and R.W. Putnam, The locus coeruleus and central chemosensitivity. Respir Physiol Neurobiol, 2010. 173(3): p. 264-73.

118. Lee, M.G., O.K. Hassani, and B.E. Jones, Discharge of identified orexin/hypocretin neurons across the sleep-waking cycle. J Neurosci, 2005. 25(28): p. 6716-20.

119. Kiyashchenko, L.I., et al., Release of hypocretin (orexin) during waking and sleep states. J Neurosci, 2002. 22(13): p. 5282-6.

120. Eggermann, E., et al., The wake-promoting hypocretin-orexin neurons are in an intrinsic state of membrane depolarization. J Neurosci, 2003. 23(5): p. 1557-62.

121. Li, A. and E. Nattie, Antagonism of rat orexin receptors by almorexant attenuates central chemoreception in wakefulness in the active period of the diurnal cycle. J Physiol, 2010. 588(Pt 15): p. 2935-44.

122. Mieda, M., et al., Differential roles of orexin receptor-1 and -2 in the regulation of nonREM and REM sleep. J Neurosci, 2011. 31(17): p. 6518-26.

123. Nattie, E. and A. Li, Respiration and autonomic regulation and orexin. Prog Brain Res, 2012. 198: p. 25-46.

124. Nakamura, A., et al., Vigilance state-dependent attenuation of hypercapnic chemoreflex and exaggerated sleep apnea in orexin knockout mice. J Appl Physiol (1985), 2007. 102(1): p. 241-8.

125. Deng, B.S., et al., Contribution of orexin in hypercapnic chemoreflex: evidence from genetic and pharmacological disruption and supplementation studies in mice. J Appl Physiol (1985), 2007. 103(5): p. 1772-9.

126. Samson, W.K., et al., Cardiovascular regulatory actions of the hypocretins in brain. Brain Res, 1999. 831(1-2): p. 248-53.

127. Chen, C.T., et al., Pressor effects of orexins injected intracisternally and to rostral ventrolateral medulla of anesthetized rats. Am J Physiol Regul Integr Comp Physiol, 2000. 278(3): p. R692-7.

128. Machado, B.H., et al., Pressor response to microinjection of orexin/hypocretin into rostral ventrolateral medulla of awake rats. Regul Pept, 2002. 104(1-3): p. 75-81.

129. Kayaba, Y., et al., Attenuated defense response and low basal blood pressure in orexin knockout mice. Am J Physiol Regul Integr Comp Physiol, 2003. 285(3): p. R581-93.

130. Li, A., et al., Antagonism of orexin receptors significantly lowers blood pressure in spontaneously hypertensive rats. J Physiol, 2013. 591(17): p. 4237-48.

131. Jacobs, B.L. and E.C. Azmitia, Structure and function of the brain serotonin system. Physiol Rev, 1992. 72(1): p. 165-229.

132. Horner, R.L., et al., Serotonin at the laterodorsal tegmental nucleus suppresses rapid-eyemovement sleep in freely behaving rats. J Neurosci, 1997. 17(19): p. 7541-52. 
133. Grace, K.P., H. Liu, and R.L. Horner, 5-HT1A receptor-responsive pedunculopontine tegmental neurons suppress REM sleep and respiratory motor activity. J Neurosci, 2012. 32(5): p. 1622-33.

134. Brown, J.W., et al., Activation of 5-HT1A receptors in medullary raphe disrupts sleep and decreases shivering during cooling in the conscious piglet. Am J Physiol Regul Integr Comp Physiol, 2008. 294(3): p. R884-94.

135. Boutrel, B., et al., Key role of 5-HT1B receptors in the regulation of paradoxical sleep as evidenced in 5-HT1B knock-out mice. J Neurosci, 1999. 19(8): p. 3204-12.

136. Monti, J.M., et al., Effects of selective activation of the 5-HT1B receptor with CP-94,253 on sleep and wakefulness in the rat. Neuropharmacology, 1995. 34(12): p. 1647-51.

137. Hedlund, P.B., et al., 5-HT7 receptor inhibition and inactivation induce antidepressantlike behavior and sleep pattern. Biol Psychiatry, 2005. 58(10): p. 831-7.

138. Monti, J.M. and H. Jantos, Effects of the 5-HT(7) receptor antagonist SB-269970 microinjected into the dorsal raphe nucleus on REM sleep in the rat. Behav Brain Res, 2006. 167(2): p. 245-50.

139. Zhao, Z.Q., et al., Lmxlb is required for maintenance of central serotonergic neurons and mice lacking central serotonergic system exhibit normal locomotor activity. J Neurosci, 2006. 26(49): p. 12781-8.

140. Buchanan, G.F., et al., 5-HT2A receptor activation is necessary for CO2-induced arousal. J Neurophysiol, 2015. 114(1): p. 233-43.

141. Buchanan, G.F. and G.B. Richerson, Central serotonin neurons are required for arousal to CO2. Proc Natl Acad Sci U S A, 2010. 107(37): p. 16354-9.

142. Young, J., M.R. Hodges, and K.J. Cummings. Role of central serotonin in sleep architecture and arousal in infant rat pups. in The Faseb Journal. 2016. Experimental Biology 2016.

143. Azmitia, E.C. and P.M. Whitaker-Azmitia, Awakening the sleeping giant: anatomy and plasticity of the brain serotonergic system. J Clin Psychiatry, 1991. 52 Suppl: p. 4-16.

144. Cote, F., et al., Recent advances in understanding serotonin regulation of cardiovascular function. Trends Mol Med, 2004. 10(5): p. 232-8.

145. Gibb, J.W., et al., Mechanisms mediating biogenic amine deficits induced by amphetamine and its congeners. NIDA Res Monogr, 1993. 136: p. 226-36; discussion 236-41.

146. Steele, T.D., U.D. McCann, and G.A. Ricaurte, 3,4-Methylenedioxymethamphetamine (MDMA, "Ecstasy"): pharmacology and toxicology in animals and humans. Addiction, 1994. 89(5): p. 539-51.

147. Kuhn, D.M. and R. Arthur, Jr., Molecular mechanism of the inactivation of tryptophan hydroxylase by nitric oxide: attack on critical sulfhydryls that spare the enzyme iron center. J Neurosci, 1997. 17(19): p. 7245-51.

148. Yang, H.T. and K.J. Cummings, Brain stem serotonin protects blood pressure in neonatal rats exposed to episodic anoxia. J Appl Physiol (1985), 2013. 115(12): p. 1733-41.

149. Holtman, J.R., Jr., et al., Evidence for 5-hydroxytryptamine, substance P, and thyrotropinreleasing hormone in neurons innervating the phrenic motor nucleus. The Journal of neuroscience : the official journal of the Society for Neuroscience, 1984. 4(4): p. 1064-71.

150. Stornetta, R.L. and P.G. Guyenet, Distribution of glutamic acid decarboxylase mRNAcontaining neurons in rat medulla projecting to thoracic spinal cord in relation to monoaminergic brainstem neurons. J Comp Neurol, 1999. 407(3): p. 367-80.

151. Halliday, G.M., et al., Distribution of substance P-like immunoreactive neurons in the human medulla oblongata: co-localization with monoamine-synthesizing neurons. Synapse, 1988. 2(4): p. 353-70.

152. Cummings, K.J., A. Li, and E.E. Nattie, Brainstem serotonin deficiency in the neonatal period: autonomic dysregulation during mild cold stress. J Physiol, 2011. 589(Pt 8): p. 2055-64. 
153. Monti, J.M., Serotonin control of sleep-wake behavior. Sleep Med Rev, 2011. 15(4): p. 269-81.

154. Mosienko, V., et al., Exaggerated aggression and decreased anxiety in mice deficient in brain serotonin. Transl Psychiatry, 2012. 2: p. e122.

155. Kaplan, K., et al., Chronic central serotonin depletion attenuates ventilation and body temperature in young but not adult Tph2 knockout rats. J Appl Physiol (1985), 2016. 120(9): p. 1070-81.

156. Magnusson, J. and K.J. Cummings, Plasticity in breathing and arterial blood pressure following acute intermittent hypercapnic hypoxia in infant rat pups with a partial loss of 5-HT neurons. Am J Physiol Regul Integr Comp Physiol, 2015. 309(10): p. R1273-84.

157. Hodges, M.R. and G.B. Richerson, Contributions of 5-HT neurons to respiratory control: neuromodulatory and trophic effects. Respir Physiol Neurobiol, 2008. 164(1-2): p. 222-32.

158. Cummings, K.J., et al., Bradycardia in serotonin-deficient Pet-1-/- mice: influence of respiratory dysfunction and hyperthermia over the first 2 postnatal weeks. Am J Physiol Regul Integr Comp Physiol, 2010. 298(5): p. R1333-42.

159. Thor, K.B., S. Nickolaus, and C.J. Helke, Autoradiographic localization of 5hydroxytryptamine1A, 5-hydroxytryptamine1B and 5-hydroxytryptamine1C/2 binding sites in the rat spinal cord. Neuroscience, 1993. 55(1): p. 235-52.

160. Gartside, S.E., et al., AMPA and NMDA receptor regulation of firing activity in 5-HT neurons of the dorsal and median raphe nuclei. The European journal of neuroscience, 2007. 25(10): p. 3001-8.

161. Judge, S.J., C.D. Ingram, and S.E. Gartside, GABA receptor modulation of 5-HT neuronal firing: characterization and effect of moderate in vivo variations in glucocorticoid levels. Neurochemistry international, 2004. 45(7): p. 1057-65.

162. Sirieix, C., et al., Role of the lateral paragigantocellular nucleus in the network of paradoxical (REM) sleep: an electrophysiological and anatomical study in the rat. PloS one, 2012. 7(1): p. e28724.

163. McCall, R.B. and M.E. Clement, Role of serotonin1A and serotonin2 receptors in the central regulation of the cardiovascular system. Pharmacol Rev, 1994. 46(3): p. 231-43.

164. Doris, P.A., Central cardiovascular regulation and the role of vasopressin: a review. Clin Exp Hypertens A, 1984. 6(12): p. 2197-217.

165. Van de Kar, L.D., Forebrain pathways mediating stress-induced renin secretion. Clin Exp Pharmacol Physiol, 1996. 23(2): p. 166-70.

166. Van de Kar, L.D., et al., Role of midbrain raphe in stress-induced renin and prolactin secretion. Brain Res, 1984. 311(2): p. 333-41.

167. Lee, K.S., et al., Serotonin inhibits GABA synaptic transmission in presympathetic paraventricular nucleus neurons. Neuroscience letters, 2008. 439(2): p. 138-42.

168. Dergacheva, O., et al., 5HT1A receptors inhibit glutamate inputs to cardiac vagal neurons post-hypoxia/hypercapnia. Respir Physiol Neurobiol, 2011. 179(2-3): p. 254-8.

169. Trueta, C. and F.F. De-Miguel, Extrasynaptic exocytosis and its mechanisms: a source of molecules mediating volume transmission in the nervous system. Front Physiol, 2012. 3: p. 319.

170. Haddjeri, N., N. Lavoie, and P. Blier, Electrophysiological evidence for the tonic activation of 5-HT(1A) autoreceptors in the rat dorsal raphe nucleus. Neuropsychopharmacology, 2004. 29(10): p. 1800-6.

171. Blakely, R.D., et al., Cloning and expression of a functional serotonin transporter from rat brain. Nature, 1991. 354(6348): p. 66-70.

172. Daws, L.C., et al., In vivo chronoamperometric measures of extracellular serotonin clearance in rat dorsal hippocampus: contribution of serotonin and norepinephrine transporters. J Pharmacol Exp Ther, 1998. 286(2): p. 967-76. 
173. Daws, L.C. and G.M. Toney, High-Speed Chronoamperometry to Study Kinetics and Mechanisms for Serotonin Clearance In Vivo, in Electrochemical Methods for Neuroscience, A.C. Michael and L.M. Borland, Editors. 2007: Boca Raton (FL).

174. Berger, M., J.A. Gray, and B.L. Roth, The expanded biology of serotonin. Annu Rev Med, 2009. 60: p. 355-66.

175. Atack, C., N.H. Bass, and P. Lundborg, Mechanisms for the elimination of 5hydroxyindoleacetic acid from brain and cerebrospinal fluid of the rat during postnatal development. Brain Res, 1974. 77(1): p. 111-20.

176. Hannon, J. and D. Hoyer, Molecular biology of 5-HT receptors. Behav Brain Res, 2008. 195(1): p. 198-213.

177. Hoyer, D., J.P. Hannon, and G.R. Martin, Molecular, pharmacological and functional diversity of 5-HT receptors. Pharmacol Biochem Behav, 2002. 71(4): p. 533-54.

178. Shayit, M., et al., 5-HT(1A) receptor subsensitivity in infancy and supersensitivity in adulthood in an animal model of depression. Brain Res, 2003. 980(1): p. 100-8.

179. Barnes, N.M. and T. Sharp, A review of central 5-HT receptors and their function. Neuropharmacology, 1999. 38(8): p. 1083-152.

180. Fink, K.B. and M. Gothert, 5-HT receptor regulation of neurotransmitter release. Pharmacol Rev, 2007. 59(4): p. 360-417.

181. McCorvy, J.D. and B.L. Roth, Structure and function of serotonin G protein-coupled receptors. Pharmacol Ther, 2015. 150: p. 129-42.

182. Filip, M. and M. Bader, Overview on 5-HT receptors and their role in physiology and pathology of the central nervous system. Pharmacol Rep, 2009. 61(5): p. 761-77.

183. Polter, A.M. and X. Li, 5-HT1A receptor-regulated signal transduction pathways in brain. Cellular signalling, 2010. 22(10): p. 1406-12.

184. Barrett, K.T., et al., Subtle alterations in breathing and heart rate control in the 5-HT1A receptor knockout mouse in early postnatal development. J Appl Physiol (1985), 2012. 113(10): p. 1585-93.

185. Messier, M.L., A. Li, and E.E. Nattie, Inhibition of medullary raphe serotonergic neurons has age-dependent effects on the CO2 response in newborn piglets. J Appl Physiol (1985), 2004. 96(5): p. 1909-19.

186. Monti, J.M., The structure of the dorsal raphe nucleus and its relevance to the regulation of sleep and wakefulness. Sleep Med Rev, 2010. 14(5): p. 307-17.

187. King, K.A. and J.R. Holtman, Jr., Characterization of the effects of activation of ventral medullary serotonin receptor subtypes on cardiovascular activity and respiratory motor outflow to the diaphragm and larynx. J Pharmacol Exp Ther, 1990. 252(2): p. 665-74.

188. Raul, L., Serotonin 2 receptors in the nucleus tractus solitarius: characterization and role in the baroreceptor reflex arc. Cell Mol Neurobiol, 2003. 23(4-5): p. 709-26.

189. Comet, M.A., et al., Activation of nucleus tractus solitarius 5-HT2A but not other 5-HT2 receptor subtypes inhibits the sympathetic activity in rats. European Journal of Neuroscience, 2007. 26(2): p. 345-354.

190. Anderson, I.K., G.R. Martin, and A.G. Ramage, Evidence that activation of 5-HT2 receptors in the forebrain of anaesthetized cats causes sympathoexcitation. $\mathrm{Br} \mathrm{J}$ Pharmacol, 1995. 116(2): p. 1751-6.

191. Devinney, M.J., et al., Hypoxia-induced phrenic long-term facilitation: emergent properties. Ann N Y Acad Sci, 2013. 1279: p. 143-53.

192. Fields, D.P. and G.S. Mitchell, Spinal metaplasticity in respiratory motor control. Front Neural Circuits, 2015. 9: p. 2.

193. Sevoz, C., et al., Role of serotonin 3 receptors in the nucleus tractus solitarii on the carotid chemoreflex. The American journal of physiology, 1997. 272(3 Pt 2): p. H1250-9.

194. Miquel, M.C., et al., Differential subcellular localization of the 5-HT3-As receptor subunit in the rat central nervous system. Eur J Neurosci, 2002. 15(3): p. 449-57. 
195. Wang, Y., A.G. Ramage, and D. Jordan, Mediation by 5-HT3 receptors of an excitatory effect of 5-HT on dorsal vagal preganglionic neurones in anaesthetized rats: an ionophoretic study. Br J Pharmacol, 1996. 118(7): p. 1697-704.

196. Monti, J.M. and H. Jantos, Activation of the serotonin 5-HT3 receptor in the dorsal raphe nucleus suppresses REM sleep in the rat. Prog Neuropsychopharmacol Biol Psychiatry, 2008. 32(4): p. 940-7.

197. Hagena, H. and D. Manahan-Vaughan, The serotonergic 5-HT4 receptor: A unique modulator of hippocampal synaptic information processing and cognition. Neurobiology of learning and memory, 2017. 138: p. 145-153.

198. Manzke, T., et al., 5-HT4(a) receptors avert opioid-induced breathing depression without loss of analgesia. Science (New York, N Y ), 2003. 301(5630): p. 226-9.

199. Watts, S.W., et al., Serotonin and blood pressure regulation. Pharmacol Rev, 2012. 64(2): p. 359-88.

200. Edwards, E. and J.F.R. Paton, 5-HT4 receptors in nucleus tractus solitarii attenuate cardiopulmonary reflex in anesthetized rats. American journal of physiology Heart and circulatory physiology, 1999. 277(5): p. H1914-H1923.

201. Grailhe, R., G.W. Grabtree, and R. Hen, Human 5-HT(5) receptors: the 5-HT(5A) receptor is functional but the 5-HT(5B) receptor was lost during mammalian evolution. European journal of pharmacology, 2001. 418(3): p. 157-67.

202. Bockaert, J., et al., Neuronal 5-HT metabotropic receptors: fine-tuning of their structure, signaling, and roles in synaptic modulation. Cell and tissue research, 2006. 326(2): p. 55372.

203. Ramage, A.G. and C.M. Villalon, 5-hydroxytryptamine and cardiovascular regulation. Trends in pharmacological sciences, 2008. 29(9): p. 472-81.

204. Pereira, M., et al., 5-HT6 receptor agonism facilitates emotional learning. Front Pharmacol, 2015. 6: p. 200.

205. Monti, J.M. and H. Jantos, Effects of the 5-HT(6) receptor antagonists SB-399885 and RO4368554 and of the 5-HT(2A) receptor antagonist EMD 281014 on sleep and wakefulness in the rat during both phases of the light-dark cycle. Behav Brain Res, 2011. 216(1): p. 381-8.

206. Hodges, M.R. and G.B. Richerson, Contributions of 5-HT neurons to respiratory control: neuromodulatory and trophic effects. Respiratory physiology \& neurobiology, 2008. 164(1-2): p. 222-32.

207. Hoffman, M.S. and G.S. Mitchell, Spinal 5-HT7 receptor activation induces long-lasting phrenic motor facilitation. J Physiol, 2011. 589(Pt 6): p. 1397-407.

208. Monti, J.M., Serotonin control of sleep-wake behavior. Sleep medicine reviews, 2011. 15(4): p. 269-81.

209. Leal, D.M., et al., Microinjection of a 5-HT3 receptor agonist into the NTS of awake rats inhibits the bradycardic response to activation of the von Bezold-Jarisch reflex. Brain Res Bull, 2001. 54(1): p. 7-11.

210. Kellett, D.O., et al., Effect of 5-HT depletion on cardiovascular vagal reflex sensitivity in awake and anesthetized rats. Brain Res, 2005. 1054(1): p. 61-72.

211. Cummings, K.J., et al., Severe spontaneous bradycardia associated with respiratory disruptions in rat pups with fewer brain stem 5-HT neurons. Am J Physiol Regul Integr Comp Physiol, 2009. 296(6): p. R1783-96.

212. Itoh, H., R.H. Alper, and R.D. Bunag, Baroreflex changes produced by serotonergic or catecholaminergic lesions in the rat nucleus tractus solitarius. J Pharmacol Exp Ther, 1992. 261(1): p. 225-33.

213. Hodges, M.R., et al., Transgenic mice lacking serotonin neurons have severe apnea and high mortality during development. J Neurosci, 2009. 29(33): p. 10341-9. 
214. Young, J.O., et al., Active sleep unmasks apnea and delayed arousal in infant rat pups lacking central serotonin. J Appl Physiol (1985), 2017. 123(4): p. 825-834.

215. Richerson, G.B., et al., Chemosensitivity of serotonergic neurons in the rostral ventral medulla. Respir Physiol, 2001. 129(1-2): p. 175-89.

216. Hodges, M.R. and G.B. Richerson, Interaction between defects in ventilatory and thermoregulatory control in mice lacking 5-HT neurons. Respir Physiol Neurobiol, 2008. 164(3): p. 350-7.

217. Hodges, M.R., S. Best, and G.B. Richerson, Altered ventilatory and thermoregulatory control in male and female adult Pet-1 null mice. Respir Physiol Neurobiol, 2011. 177(2): p. 133-40.

218. Hickner, S., et al., Ventilatory long-term facilitation is evident after initial and repeated exposure to intermittent hypoxia in mice genetically depleted of brain serotonin. J Appl Physiol (1985), 2014. 116(3): p. 240-50.

219. Chen, J., et al., Time- and age-dependent effects of serotonin on gasping and autoresuscitation in neonatal mice. J Appl Physiol (1985), 2013. 114(12): p. 1668-76.

220. Gershan, W.M., M.S. Jacobi, and B.T. Thach, Maturation of Cardiorespiratory Interactions in Spontaneous-Recovery from Hypoxic Apnea (Autoresuscitation). Pediatric Research, 1990. 28(2): p. 87-93.

221. Kinney, H.C. and B.T. Thach, The sudden infant death syndrome. N Engl J Med, 2009. 361(8): p. 795-805.

222. Kinney, H.C., et al., The Brainstem and Serotonin in the Sudden Infant Death Syndrome. Annual Review of Pathology-Mechanisms of Disease, 2009. 4: p. 517-550.

223. McGinty, D.J. and R.M. Harper, Dorsal raphe neurons: depression of firing during sleep in cats. Brain Res, 1976. 101(3): p. 569-75.

224. Alenina, N., et al., Growth retardation and altered autonomic control in mice lacking brain serotonin. Proc Natl Acad Sci U S A, 2009. 106(25): p. 10332-7.

225. Young, J.O., et al., Active sleep unmasks apnea and delayed arousal in infant rat pups lacking central serotonin. J Appl Physiol (1985), 2017: p. jap 004392017.

226. Baker, T.L. and G.S. Mitchell, Episodic but not continuous hypoxia elicits long-term facilitation of phrenic motor output in rats. J Physiol, 2000. 529 Pt 1: p. 215-9.

227. Xing, T., P.M. Pilowsky, and A.Y. Fong, Mechanism of sympathetic activation and blood pressure elevation in humans and animals following acute intermittent hypoxia. Prog Brain Res, 2014. 209: p. 131-46.

228. Nakamura, A., et al., Sleep state dependence of ventilatory long-term facilitation following acute intermittent hypoxia in Lewis rats. J Appl Physiol (1985), 2010. 109(2): p. 323-31.

229. Nichols, N.L., E.A. Dale, and G.S. Mitchell, Severe acute intermittent hypoxia elicits phrenic long-term facilitation by a novel adenosine-dependent mechanism. J Appl Physiol (1985), 2012. 112(10): p. 1678-88.

230. Golder, F.J., et al., Spinal adenosine A2a receptor activation elicits long-lasting phrenic motor facilitation. J Neurosci, 2008. 28(9): p. 2033-42.

231. McKay, L.C., W.A. Janczewski, and J.L. Feldman, Episodic hypoxia evokes long-term facilitation of genioglossus muscle activity in neonatal rats. J Physiol, 2004. 557(Pt 1): p. 13-8.

232. Fuller, D.D., et al., Prenatal nicotine exposure alters respiratory long-term facilitation in neonatal rats. Respir Physiol Neurobiol, 2009. 169(3): p. 333-7.

233. Lechin, F. and B. van der Dijs, Central nervous system circuitry and peripheral neural sympathetic activity responsible for essential hypertension. Curr Neurovasc Res, 2006. 3(4): p. 307-25.

234. Koulu, M., et al., Association between increased serotonin metabolism in rat brainstem nuclei and development of spontaneous hypertension. Brain research, 1986. 371(1): p. 17781. 
235. Buckingham, R.E., T.C. Hamilton, and D. Robson, Effect of intracerebroventricular 5,6dihydroxytryptamine on blood pressure of spontaneously hypertensive rats. European journal of pharmacology, 1976. 36(2): p. 431-7.

236. Kinney, H.C., et al., The brainstem and serotonin in the sudden infant death syndrome. Annu Rev Pathol, 2009. 4: p. 517-50.

237. Randall, B.B., et al., Potential asphyxia and brainstem abnormalities in sudden and unexpected death in infants. Pediatrics, 2013. 132(6): p. e1616-25.

238. Kung, L.H. and K.E. Scrogin, Serotonin nerve terminals in the dorsomedial medulla facilitate sympathetic and ventilatory responses to hemorrhage and peripheral chemoreflex activation. Am J Physiol Regul Integr Comp Physiol, 2011. 301(5): p. R136779.

239. Steinbusch, H.W., Distribution of serotonin-immunoreactivity in the central nervous system of the rat-cell bodies and terminals. Neuroscience, 1981. 6(4): p. 557-618.

240. Paterson, D.S., G. Hilaire, and D.E. Weese-Mayer, Medullary serotonin defects and respiratory dysfunction in sudden infant death syndrome. Respir Physiol Neurobiol, 2009. 168(1-2): p. 133-43.

241. Carretero, O.A. and S. Oparil, Essential hypertension. Part I: definition and etiology. Circulation, 2000. 101(3): p. 329-35.

242. Oparil, S., M.A. Zaman, and D.A. Calhoun, Pathogenesis of hypertension. Ann Intern Med, 2003. 139(9): p. 761-76.

243. Higashi, Y., et al., Circadian variation of blood pressure and endothelial function in patients with essential hypertension: a comparison of dippers and non-dippers. J Am Coll Cardiol, 2002. 40(11): p. 2039-43.

244. Muller, J.E., et al., Circadian variation in the frequency of onset of acute myocardial infarction. N Engl J Med, 1985. 313(21): p. 1315-22.

245. Smolensky, M.H. and E. Haus, Circadian rhythms and clinical medicine with applications to hypertension. Am J Hypertens, 2001. 14(9 Pt 2): p. 280S-290S.

246. Zulley, J., Distribution of REM sleep in entrained 24 hour and free-running sleep--wake cycles. Sleep, 1980. 2(4): p. 377-89.

247. Berteotti, C., et al., Surges of arterial pressure during REM sleep in spontaneously hypertensive rats. Sleep, 2008. 31(1): p. 111-7.

248. Villalon, C.M., P. deVries, and P.R. Saxena, Serotonin receptors as cardiovascular targets. Drug Discovery Today, 1997. 2(7): p. 294-300.

249. Szewczak, J.M. and F.L. Powell, Open-flow plethysmography with pressure-decay compensation. Respir Physiol Neurobiol, 2003. 134(1): p. 57-67.

250. Criee, C.P., et al., Body plethysmography--its principles and clinical use. Respir Med, 2011. 105(7): p. 959-71.

251. $\mathrm{Li}, \mathrm{A}$. and $\mathrm{E}$. Nattie, Catecholamine neurones in rats modulate sleep, breathing, central chemoreception and breathing variability. J Physiol, 2006. 570(Pt 2): p. 385-96.

252. Cirelli, C. and G. Tononi, Cortical development, electroencephalogram rhythms, and the sleep/wake cycle. Biol Psychiatry, 2015. 77(12): p. 1071-8.

253. Julien, C., A. Bairam, and V. Joseph, Chronic intermittent hypoxia reduces ventilatory long-term facilitation and enhances apnea frequency in newborn rats. Am J Physiol Regul Integr Comp Physiol, 2008. 294(4): p. R1356-66.

254. Duncan, J.R., et al., Brainstem serotonergic deficiency in sudden infant death syndrome. JAMA, 2010. 303(5): p. 430-7.

255. Paterson, D.S., et al., Multiple serotonergic brainstem abnormalities in sudden infant death syndrome. JAMA, 2006. 296(17): p. 2124-32.

256. Schechtman, V.L., et al., Cardiac and respiratory patterns in normal infants and victims of the sudden infant death syndrome. Sleep, 1988. 11(5): p. 413-24. 
257. Schechtman, V.L., et al., Heart rate variation in normal infants and victims of the sudden infant death syndrome. Early Hum Dev, 1989. 19(3): p. 167-81.

258. Marshall, J.M. and J.D. Metcalfe, Influences on the cardiovascular response to graded levels of systemic hypoxia of the accompanying hypocapnia in the rat. J Physiol, 1989. 410: p. 381-94.

259. King, T.L., et al., Hypoxia activates nucleus tractus solitarii neurons projecting to the paraventricular nucleus of the hypothalamus. Am J Physiol Regul Integr Comp Physiol, 2012. 302(10): p. R1219-32.

260. Walker, B.R. and B.L. Brizzee, Cardiovascular responses to hypoxia and hypercapnia in barodenervated rats. J Appl Physiol (1985), 1990. 68(2): p. 678-86.

261. Oikawa, S., et al., Autonomic cardiovascular responses to hypercapnia in conscious rats: the roles of the chemo- and baroreceptors. Auton Neurosci, 2005. 117(2): p. 105-14.

262. Pladys, P., et al., Influence of prematurity on postnatal maturation of heart rate and arterial pressure responses to hypoxia in lambs. Neonatology, 2008. 93(3): p. 197-205.

263. Sidi, D., et al., Developmental changes in oxygenation and circulatory responses to hypoxemia in lambs. Am J Physiol, 1983. 245(4): p. H674-82.

264. Xing, T., et al., Acute intermittent hypoxia induced neural plasticity in respiratory motor control. Clin Exp Pharmacol Physiol, 2013. 40(9): p. 602-9.

265. Ling, L., et al., Chronic intermittent hypoxia elicits serotonin-dependent plasticity in the central neural control of breathing. J Neurosci, 2001. 21(14): p. 5381-8.

266. Dick, T.E., et al., Acute intermittent hypoxia increases both phrenic and sympathetic nerve activities in the rat. Exp Physiol, 2007. 92(1): p. 87-97.

267. Poets, C.F., Apparent life-threatening events and sudden infant death on a monitor. Paediatr Respir Rev, 2004. 5 Suppl A: p. S383-6.

268. Meny, R.G., et al., Cardiorespiratory recordings from infants dying suddenly and unexpectedly at home. Pediatrics, 1994. 93(1): p. 44-9.

269. Cummings, K.J., et al., Postnatal loss of brainstem serotonin neurones compromises the ability of neonatal rats to survive episodic severe hypoxia. J Physiol, 2011. 589(Pt 21): p. 5247-56.

270. Drorbaugh, J.E. and W.O. Fenn, A barometric method for measuring ventilation in newborn infants. Pediatrics, 1955. 16(1): p. 81-7.

271. Paxinos, G. and C. Watson, The rat brain in stereotaxic coordinates. 6th ed. 2007, Amsterdam ; Boston ;: Academic Press/Elsevier.

272. Hofer, M.A., J.P. Leaf, and H.N. Shair, Baroreceptor sensitivity in two-week-old rat pups: effects of nutrient deprivation and sino-aortic denervation. Physiol Behav, 1988. 43(6): p. 823-30.

273. Mandal, A.K., et al., Stimulation of serotonin2 receptors in the ventrolateral medulla of the cat results in nonuniform increases in sympathetic outflow. Circ Res, 1990. 67(5): p. 1267-80.

274. Izzo, P.N., J. Deuchars, and K.M. Spyer, Localization of cardiac vagal preganglionic motoneurones in the rat: immunocytochemical evidence of synaptic inputs containing 5hydroxytryptamine. J Comp Neurol, 1993. 327(4): p. 572-83.

275. Callera, J.C., et al., Microinjection of a serotonin3 receptor agonist into the NTS of unanesthetized rats inhibits the bradycardia evoked by activation of the baro- and chemoreflexes. J Auton Nerv Syst, 1997. 63(3): p. 127-36.

276. Reid, I.M. and I.C. Solomon, Intermittent hypoxia-induced respiratory long-term facilitation is dominated by enhanced burst frequency, not amplitude, in spontaneously breathing urethane-anesthetized neonatal rats. Prog Brain Res, 2014. 212: p. 221-35.

277. Dempsey, J.A., et al., The ventilatory responsiveness to CO(2) below eupnoea as a determinant of ventilatory stability in sleep. J Physiol, 2004. 560(Pt 1): p. 1-11. 
278. Hoffman, M.S. and G.S. Mitchell, Spinal 5-HT7 receptors and protein kinase A constrain intermittent hypoxia-induced phrenic long-term facilitation. Neuroscience, 2013. 250: p. $632-43$.

279. Hoffman, M.S., et al., Spinal adenosine A2(A) receptor inhibition enhances phrenic long term facilitation following acute intermittent hypoxia. J Physiol, 2010. 588(Pt 1): p. 25566.

280. Kc, P. and T.E. Dick, Modulation of cardiorespiratory function mediated by the paraventricular nucleus. Respir Physiol Neurobiol, 2010. 174(1-2): p. 55-64.

281. Leiter, J.C., Serotonin, gasping, autoresuscitation, and SIDS--a contrarian view. J Appl Physiol (1985), 2009. 106(6): p. 1761-2.

282. Poets, C.F., et al., Gasping and other cardiorespiratory patterns during sudden infant deaths. Pediatr Res, 1999. 45(3): p. 350-4.

283. Sridhar, R., et al., Characterization of successful and failed autoresuscitation in human infants, including those dying of SIDS. Pediatr Pulmonol, 2003. 36(2): p. 113-22.

284. Ramage, A.G., Central cardiovascular regulation and 5-hydroxytryptamine receptors. Brain Res Bull, 2001. 56(5): p. 425-39.

285. Semple, B.D., et al., Brain development in rodents and humans: Identifying benchmarks of maturation and vulnerability to injury across species. Prog Neurobiol, 2013. 106-107: p. $1-16$.

286. Darnall, R.A., et al., Arousal from sleep in response to intermittent hypoxia in rat pups is modulated by medullary raphe GABAergic mechanisms. Am J Physiol Regul Integr Comp Physiol, 2012. 302(5): p. R551-60.

287. Mortola, J.P. and L. Naso, Thermogenesis in newborn rats after prenatal or postnatal hypoxia. J Appl Physiol (1985), 1998. 85(1): p. 84-90.

288. Ramage, A.G. and M.B. Daly, The central action of the 5-HT2 receptor agonist 1-(2,5dimethoxy-4-iodophenyl)-2-aminopropane (DOI) on cardiac inotropy and vascular resistance in the anaesthetized cat. Br J Pharmacol, 1998. 125(6): p. 1172-9.

289. Costarella, C.E., et al., Testosterone causes direct relaxation of rat thoracic aorta. J Pharmacol Exp Ther, 1996. 277(1): p. 34-9.

290. Dohler, K.D. and W. Wuttke, Changes with age in levels of serum gonadotropins, prolactin and gonadal steroids in prepubertal male and female rats. Endocrinology, 1975. 97(4): p. 898-907.

291. Hay, M., Sex, the brain and hypertension: brain oestrogen receptors and high blood pressure risk factors. Clin Sci (Lond), 2016. 130(1): p. 9-18.

292. Brailoiu, G.C., et al., Bradycardic effects mediated by activation of $G$ protein-coupled estrogen receptor in rat nucleus ambiguus. Exp Physiol, 2013. 98(3): p. 679-91.

293. Schechtman, V.L., et al., Dynamic analysis of cardiac $R-R$ intervals in normal infants and in infants who subsequently succumbed to the sudden infant death syndrome. Pediatr Res, 1992. 31(6): p. 606-12.

294. Narboux-Neme, N., et al., Postnatal growth defects in mice with constitutive depletion of central serotonin. ACS Chem Neurosci, 2013. 4(1): p. 171-81.

295. Kuhn, D.M., W.A. Wolf, and W. Lovenberg, Review of the role of the central serotonergic neuronal system in blood pressure regulation. Hypertension, 1980. 2(3): p. 243-55.

296. Mistrova, E., P. Kruzliak, and M. Chottova Dvorakova, Role of substance $P$ in the cardiovascular system. Neuropeptides, 2016. 58: p. 41-51.

297. Paakkari, I., et al., Injection of thyrotropin releasing hormone into the locus coeruleus increases blood pressure. Eur Heart J, 1987. 8 Suppl B: p. 147-51.

298. Magnusson, J.L. and K.J. Cummings, Central serotonin and the control of arterial blood pressure and heart rate in infant rats: influence of sleep state and sex. Am J Physiol Regul Integr Comp Physiol, 2017: p. ajpregu 003212017. 
299. Mary, D.A. and J.B. Stoker, The activity of single vasoconstrictor nerve units in hypertension. Acta Physiol Scand, 2003. 177(3): p. 367-76.

300. Li, A.H., et al., Antagonism of orexin receptors significantly lowers blood pressure in spontaneously hypertensive rats. Journal of Physiology-London, 2013. 591(17): p. 42374248.

301. Pagani, M., et al., Power spectral analysis of heart rate and arterial pressure variabilities as a marker of sympatho-vagal interaction in man and conscious dog. Circ Res, 1986. 59(2): p. 178-93.

302. Zoccal, D.B., et al., Increased sympathetic activity in rats submitted to chronic intermittent hypoxia. Exp Physiol, 2007. 92(1): p. 79-85.

303. Monti, J.M. and H. Jantos, The roles of dopamine and serotonin, and of their receptors, in regulating sleep and waking. Prog Brain Res, 2008. 172: p. 625-46.

304. Honda, T. and K. Semba, Serotonergic synaptic input to cholinergic neurons in the rat mesopontine tegmentum. Brain Res, 1994. 647(2): p. 299-306.

305. Thakkar, M.M., R.E. Strecker, and R.W. McCarley, Behavioral state control through differential serotonergic inhibition in the mesopontine cholinergic nuclei: a simultaneous unit recording and microdialysis study. J Neurosci, 1998. 18(14): p. 5490-7.

306. Morilak, D.A. and R.D. Ciaranello, 5-HT2 receptor immunoreactivity on cholinergic neurons of the pontomesencephalic tegmentum shown by double immunofluorescence. Brain Res, 1993. 627(1): p. 49-54.

307. Amici, R., et al., A serotonergic (5-HT2) receptor mechanism in the laterodorsal tegmental nucleus participates in regulating the pattern of rapid-eye-movement sleep occurrence in the rat. Brain Res, 2004. 996(1): p. 9-18.

308. Kunes, J., et al., Critical developmental periods in the pathogenesis of hypertension. Physiol Res, 2012. 61 Suppl 1: p. S9-17.

309. Nosjean, A. and P.G. Guyenet, Role of ventrolateral medulla in sympatholytic effect of 8OHDPAT in rats. The American journal of physiology, 1991. 260(3 Pt 2): p. R600-9.

310. Santajuliana, D., B.J. Hornfeldt, and J.W. Osborn, Use of ganglionic blockers to assess neurogenic pressor activity in conscious rats. J Pharmacol Toxicol Methods, 1996. 35(1): p. $45-54$.

311. Stauss, H.M., et al., Does low frequency power of arterial blood pressure reflect sympathetic tone? Journal of the autonomic nervous system, 1995. 54(2): p. 145-54.

312. Head, G.A., et al., Rate of morning increase in blood pressure is elevated in hypertensives. Am J Hypertens, 2006. 19(10): p. 1010-7.

313. Kario, K., Morning surge in blood pressure and cardiovascular risk: evidence and perspectives. Hypertension, 2010. 56(5): p. 765-73.

314. Sei, H., Blood pressure surges in REM sleep: A mini review. Pathophysiology, 2012. 19(4): p. 233-41.

315. Sei, H., et al., Injection of 6-hydroxydopamine into the ventral tegmental area suppresses the increase in arterial pressure during REM sleep in the rat. Sleep research online: SRO, 1999. 2(1): p. 1-6.

316. Ugedo, L., J. Grenhoff, and T.H. Svensson, Ritanserin, a 5-HT2 receptor antagonist, activates midbrain dopamine neurons by blocking serotonergic inhibition. Psychopharmacology, 1989. 98(1): p. 45-50.

317. Salgado, H.C., et al., Baroreflex responses to electrical stimulation of aortic depressor nerve in conscious SHR. Am J Physiol Heart Circ Physiol, 2007. 292(1): p. H593-600.

318. Parmer, R.J., J.H. Cervenka, and R.A. Stone, Baroreflex sensitivity and heredity in essential hypertension. Circulation, 1992. 85(2): p. 497-503.

319. Sved, A.F., A.M. Schreihofer, and C.K. Kost, Jr., Blood pressure regulation in baroreceptor-denervated rats. Clin Exp Pharmacol Physiol, 1997. 24(1): p. 77-82. 
320. Xue, B., A.K. Johnson, and M. Hay, Sex differences in angiotensin II- and aldosteroneinduced hypertension: the central protective effects of estrogen. Am J Physiol Regul Integr Comp Physiol, 2013. 305(5): p. R459-63.

321. Saleh, M.C., B.J. Connell, and T.M. Saleh, Autonomic and cardiovascular reflex responses to central estrogen injection in ovariectomized female rats. Brain Res, 2000. 879(1-2): p. 105-14.

322. Scrogin, K.E., A.K. Johnson, and H.A. Schmid, Multiple receptor subtypes mediate the effects of serotonin on rat subfornical organ neurons. Am J Physiol, 1998. 275(6 Pt 2): p. R2035-42.

323. Jorgensen, H.S., Studies on the neuroendocrine role of serotonin. Dan Med Bull, 2007. 54(4): p. 266-88.

324. Bethea, C.L., et al., Ovarian steroids and serotonin neural function. Mol Neurobiol, 1998. 18(2): p. 87-123.

325. Birzniece, V., et al., Neuroactive steroid effects on cognitive functions with a focus on the serotonin and GABA systems. Brain Res Rev, 2006. 51(2): p. 212-39.

326. Pecins-Thompson, M., et al., Ovarian steroid regulation of tryptophan hydroxylase mRNA expression in rhesus macaques. J Neurosci, 1996. 16(21): p. 7021-9.

327. Johansson, B., V. Georgiev, and B.B. Fredholm, Distribution and postnatal ontogeny of adenosine A2A receptors in rat brain: comparison with dopamine receptors. Neuroscience, 1997. 80(4): p. 1187-207.

328. Zaidi, S.I., et al., Adenosine A2A receptors are expressed by GABAergic neurons of medulla oblongata in developing rat. Brain Res, 2006. 1071(1): p. 42-53.

329. Ameer, O.Z., C.M. Hildreth, and J.K. Phillips, Sympathetic overactivity prevails over the vascular amplifier phenomena in a chronic kidney disease rat model of hypertension. Physiol Rep, 2014. 2(11).

330. Coldren, K.M., et al., Relaxin increases sympathetic nerve activity and activates spinally projecting neurons in the paraventricular nucleus of nonpregnant, but not pregnant, rats. American journal of physiology Regulatory, integrative and comparative physiology, 2015. 309(12): p. R1553-68.

331. Berecek, K.H. and B.H. Swords, Central role for vasopressin in cardiovascular regulation and the pathogenesis of hypertension. Hypertension, 1990. 16(3): p. 213-24.

332. Quigley, K.S., M.M. Myers, and H.N. Shair, Development of the baroreflex in the young rat. Auton Neurosci, 2005. 121(1-2): p. 26-32.

333. Magnusson, J.L. and K.J. Cummings, Central serotonin and the control of arterial blood pressure and heart rate in infant rats: influence of sleep state and sex. Am J Physiol Regul Integr Comp Physiol, 2018. 314(2): p. R313-R321.

334. Dreteler, G.H., et al., Pressor effects following microinjection of 5-HT1A receptor agonists into the raphe obscurus of the anaesthetized rat. Br J Pharmacol, 1991. 102(2): p. 317-22.

335. Broadbelt, K.G., et al., Neuroanatomic relationships between the GABAergic and serotonergic systems in the developing human medulla. Autonomic neuroscience : basic \& clinical, 2010. 154(1-2): p. 30-41.

336. Yasui, Y., D.F. Cechetto, and C.B. Saper, Evidence for a cholinergic projection from the pedunculopontine tegmental nucleus to the rostral ventrolateral medulla in the rat. Brain Res, 1990. 517(1-2): p. 19-24.

337. Cornford, E.M., et al., Comparison of lipid-mediated blood-brain-barrier penetrability in neonates and adults. Am J Physiol, 1982. 243(3): p. C161-8.

338. Banks, W.A., Brain meets body: the blood-brain barrier as an endocrine interface. Endocrinology, 2012. 153(9): p. 4111-9.

339. Dergacheva, O., et al., The lateral paragigantocellular nucleus modulates parasympathetic cardiac neurons: a mechanism for rapid eye movement sleep-dependent changes in heart rate. J Neurophysiol, 2010. 104(2): p. 685-94. 
340. Mohrman, D.E. and L.J. Heller, Cardiovascular physiology. 2014, McGraw-Hill, Education/Medical,: New York. p. ix, 276 pages.

341. Mikulic, L.E., et al., Effect of central serotonin depletion on blood pressure and the renin system in rats. Hypertension, 1988. 11(2 Pt 2): p. I190-3.

342. Prager-Khoutorsky, M., et al., Role of Vasopressin in Rat Models of Salt-Dependent Hypertension. Curr Hypertens Rep, 2017. 19(5): p. 42.

343. Ma, X., F.M. Abboud, and M.W. Chapleau, Analysis of afferent, central, and efferent components of the baroreceptor reflex in mice. Am J Physiol Regul Integr Comp Physiol, 2002. 283(5): p. R1033-40.

344. Schiffrin, E.L., Vascular remodeling in hypertension: mechanisms and treatment. Hypertension, 2012. 59(2): p. 367-74.

345. Grassi, G., et al., Baroreflex control of sympathetic nerve activity in essential and secondary hypertension. Hypertension (Dallas, Tex : 1979), 1998. 31(1): p. 68-72.

346. Nosjean, A. and P.G. Guyenet, Role of ventrolateral medulla in sympatholytic effect of 8OHDPAT in rats. Am J Physiol, 1991. 260(3 Pt 2): p. R600-9.

347. Scopinho, A.A., et al., Dorsal and ventral hippocampus modulate autonomic responses but not behavioral consequences associated to acute restraint stress in rats. PLoS One, 2013. 8(10): p. e77750.

348. Sawchenko, P.E., et al., The distribution and cells of origin of serotonergic inputs to the paraventricular and supraoptic nuclei of the rat. Brain Res, 1983. 277(2): p. 355-60.

349. Steptoe, A. and M. Kivimaki, Stress and cardiovascular disease. Nat Rev Cardiol, 2012. 9(6): p. 360-70.

350. Parker, K.J., A.F. Schatzberg, and D.M. Lyons, Neuroendocrine aspects of hypercortisolism in major depression. Horm Behav, 2003. 43(1): p. 60-6.

351. Rubio-Guerra, A.F., et al., Depression increases the risk for uncontrolled hypertension. Exp Clin Cardiol, 2013. 18(1): p. 10-2.

352. Beery, A.K. and D. Kaufer, Stress, social behavior, and resilience: insights from rodents. Neurobiol Stress, 2015. 1: p. 116-127.

353. Castelhano-Carlos, M.J. and V. Baumans, The impact of light, noise, cage cleaning and inhouse transport on welfare and stress of laboratory rats. Lab Anim, 2009. 43(4): p. 31127.

354. Genest, S.E., et al., Neonatal maternal separation and sex-specific plasticity of the hypoxic ventilatory response in awake rat. J Physiol, 2004. 554(Pt 2): p. 543-57.

355. Grippo, A.J. and A.K. Johnson, Stress, depression and cardiovascular dysregulation: a review of neurobiological mechanisms and the integration of research from preclinical disease models. Stress, 2009. 12(1): p. 1-21.

356. Whitworth, J.A., G.J. Mangos, and J.J. Kelly, Cushing, cortisol, and cardiovascular disease. Hypertension, 2000. 36(5): p. 912-6.

357. Goodwin, J.E. and D.S. Geller, Glucocorticoid-induced hypertension. Pediatr Nephrol, 2012. 27(7): p. 1059-66.

358. Heydendael, W. and L. Jacobson, Widespread hypothalamic-pituitary-adrenocortical axisrelevant and mood-relevant effects of chronic fluoxetine treatment on glucocorticoid receptor gene expression in mice. Eur J Neurosci, 2010. 31(5): p. 892-902.

359. Mitchell, J.B., et al., Serotonin regulates type II corticosteroid receptor binding in hippocampal cell cultures. J Neurosci, 1990. 10(6): p. 1745-52.

360. Laplante, P., J. Diorio, and M.J. Meaney, Serotonin regulates hippocampal glucocorticoid receptor expression via a 5-HT7 receptor. Brain Res Dev Brain Res, 2002. 139(2): p. 199203.

361. Saphier, D. and K. Zhang, Inhibition by the serotonin1A agonist, 8-hydroxy-2- (di-npropylamino)tetralin, of antidromically identified paraventricular nucleus neurons in the rat. Brain research, 1993. 615(1): p. 7-12. 
362. Zhou, J., et al., Effects of serotonin depletion on the hippocampal GR/MR and BDNF expression during the stress adaptation. Behav Brain Res, 2008. 195(1): p. 129-38.

363. Seckl, J.R., K.L. Dickson, and G. Fink, Central 5,7-dihydroxytryptamine lesions decrease hippocampal glucocorticoid and mineralocorticoid receptor messenger ribonucleic Acid expression. J Neuroendocrinol, 1990. 2(6): p. 911-6.

364. Magnusson, J.L. and K.J. Cummings, Central serotonin and the control of arterial blood pressure and heart rate in infant rats: influence of sleep state and sex. Am J Physiol Regul Integr Comp Physiol, 2017: p. ajpregu003212017.

365. Day, M.D. and A.G. Roach, Cardiovascular effects of dopamine after central administration into conscious cats. British journal of pharmacology, 1976. 58(4): p. 50515.

366. Louis, W.J., B. Jarrott, and A.E. Doyle, The role of noradrenaline and other transmitter hormones in the pathogenesis of hypertension. Clinical science and molecular medicine Supplement, 1976. 3: p. 427s-430s.

367. Berger, T.W., et al., Hyperinnervation of the striatum by dorsal raphe afferents after dopamine-depleting brain lesions in neonatal rats. Brain research, 1985. 336(2): p. 354-8.

368. Prinz, A., et al., Increased excitability in serotonin neurons in the dorsal raphe nucleus in the 6-OHDA mouse model of Parkinson's disease. Exp Neurol, 2013. 248: p. 236-45.

369. Galeeva, A., et al., Postnatal ontogeny of the glucocorticoid receptor in the hippocampus. Vitam Horm, 2010. 82: p. 367-89.

370. Pryce, C.R., Postnatal ontogeny of expression of the corticosteroid receptor genes in mammalian brains: inter-species and intra-species differences. Brain Res Rev, 2008. 57(2): p. 596-605.

371. Rood, B.D., et al., Dorsal raphe serotonin neurons in mice: immature hyperexcitability transitions to adult state during first three postnatal weeks suggesting sensitive period for environmental perturbation. J Neurosci, 2014. 34(14): p. 4809-21.

372. Mitchell, J.B., L.J. Iny, and M.J. Meaney, The role of serotonin in the development and environmental regulation of type II corticosteroid receptor binding in rat hippocampus. Brain Res Dev Brain Res, 1990. 55(2): p. 231-5.

373. Araragi, N., et al., Conservation of 5-HT1A receptor-mediated autoinhibition of serotonin (5-HT) neurons in mice with altered 5-HT homeostasis. Front Pharmacol, 2013. 4: p. 97.

374. Vasan, R.S. and D. Levy, The role of hypertension in the pathogenesis of heart failure. A clinical mechanistic overview. Arch Intern Med, 1996. 156(16): p. 1789-96.

375. Hay, M., B. Xue, and A.K. Johnson, Yes! Sex matters: sex, the brain and blood pressure. Current hypertension reports, 2014. 16(8): p. 458.

376. Brooks, V.L., et al., Baroreflex function in females: changes with the reproductive cycle and pregnancy. Gend Med, 2012.9(2): p. 61-7.

377. Mohamed, M.K., M.M. El-Mas, and A.A. Abdel-Rahman, Estrogen enhancement of baroreflex sensitivity is centrally mediated. American journal of physiology Regulatory, integrative and comparative physiology, 1999. 276(4): p. R1030-R1037. 
VITA

Jennifer L. Magnusson, the daughter of John and Cheryl Magnusson, was born in La Mesa, CA on August 24, 1984. She completed her Bachelor of Science in Biology in 2007 at Texas Tech University in Lubbock, TX. Following her undergraduate studies, she became a full-time employee at the University of Southwestern Medical Center in Dallas, TX, where she worked in the Department of Prognostic Radiology, assessing the efficacy of cancer chemotherapeutics. She joined the Biomedical Sciences Department in 2012 and has done her Ph.D. training in the laboratory of Dr. Kevin. J. Cummings. 